UNIVERSITE DU QUEBEC A CHICOUTIMI

\author{
MEMOIRE \\ PRESENTE A \\ L'UNIVERSITE DU QUEBEC A CHICOUTIMI \\ COMME EXIGENCE PARTIELLE \\ DE LA MAITRISE EN ETUDES REGIONALES
}

PAR

ALAIN ROUSSEAU

B. SC. SC. POLIT.

\begin{abstract}
ARCHIPEL BOLAMA-BIJAGOS EN GUINEE-BISSAU;
POPULATION LOCALE ET DEVELOPPEMENT PLANIFIE
\end{abstract}

AOUT 1990 


\section{Bibliothèque}

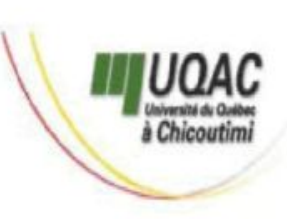

\section{Mise en garde/Advice}

Afin de rendre accessible au plus Motivated by a desire to make the grand nombre le résultat des results of its graduate students' travaux de recherche menés par ses research accessible to all, and in étudiants gradués et dans l'esprit des accordance with the rules règles qui régissent le dépôt et la governing the acceptation and diffusion des mémoires et thèses diffusion of dissertations and produits dans cette Institution, theses in this Institution, the I'Université du Québec à Université du Québec à Chicoutimi (UQAC) est fière de Chicoutimi (UQAC) is proud to rendre accessible une version make a complete version of this complète et gratuite de cette œuvre. work available at no cost to the reader.

L'auteur conserve néanmoins la The author retains ownership of the propriété du droit d'auteur qui copyright of this dissertation or protège ce mémoire ou cette thèse. thesis. Neither the dissertation or Ni le mémoire ou la thèse ni des thesis, nor substantial extracts from extraits substantiels de ceux-ci ne it, may be printed or otherwise peuvent être imprimés ou autrement reproduced without the author's reproduits sans son autorisation. permission. 


\section{RESUME}

Le mémoire recherche les avenues possibles de développement d'une île, Bolama, de l'archipel Bolama-Bijagos en Guinée-Bissau.

A partir de la constatation que lî̀le constitue en soi une région parce qu'elle forme un espace homogène, distinct et original par rapport au reste du pays, la recherche insiste sur les particularités humaines propres à ce territoire, après avoir dressé un bref portrait historique et socio-économique du pays. A ce niveau, le mémoire s'attarde brièvement sur l'aspect physique tant du pays, que de la région Bolama-Bijagos.

L'originalité de Bolama réside dans le fait qu'elle forme une société duale composée de deux mondes, l'un rural, l'autre urbain, différents sur de nombreux points. Le monde rural, partagé entre un mode de production communautaire et un mode de production tributaire, vit, à des degrés divers, sous une économie de subsistance. La ville, par contre, est fortement engagé dans l'économie monétaire et, sur de nombreux points, elle affiche les traits des villes du tiers-monde, coupées de leur arrière-pays et dépendante des ressources du pays et de l'aide internationale tant pour leur alimentation que leur fonctionnement.

Après une première partie descriptive dressant le portrait de Bolama en regard de son milieu physique, du profil ethnique de sa population et du survol de sa culture et de ses activités de production, le mémoire insiste, dans un deuxième temps, sur l'analyse du système de planification national et, tout particulièrement, de son volet régional. Ainsi, l'étude des modes de production, des ethnies, de l'agriculture, de la pêche, des spécialisations ethniques et sexuelles, des projets de coopération, des structures politicoadministratives nationales et régionales, et des deux plans quadriennaux de développement, figure à l'ordre du jour du document.

Toute cette analyse s'effectuera en tenant compte d'une définition du développement qui l'associe au processus et à la capacité d'une société et d'une population de prendre en charge les différents aspects de ses forces productives en vue d'une amélioration de son bien-être en général. Qui plus est, ce développement au "ras du sol" doit être intégré afin d'émaner directement de la population concernée. Analysées sous cette grille, les actions des différents intervenants socio-économiques nationaux et étrangers seront définies par rapport aux réalités ethno-culturelles locales et aux aspiration et besoins exprimés lors d'une enquête dans les villages, par des membres de ces communautés. 
iii

Le mémoire entend donc ainsi devenir une source de référence sur Bolama et un outil d'analyse du modèle de développement planifié de l'Etat bissau-guinéen et de son Parti, le P.A.I.G.C., et de la pratique des projets de coopération qui interviennent dans le milieu.

Par la richesse ethno-culturelle de Bolama, l'ouvrage aspire, sous certaines réserves, à pouvoir être appliqué aux autres îles de l'archipel et à d'autres régions du pays ou même de l'Afrique, jouissant d'un contexte similaire.

OXain Pawnowa

Alain Rousseau

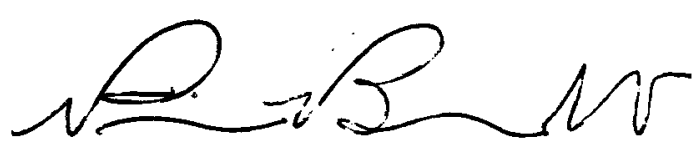

Pierre W. Boudreault Directeur de recherche 


\section{AVANT-PROPOS}

Cette recherche a été rendue possible grâce à une expérience de deux ans (de juillet 1986 a juillet 1988) en tant que coopérant-volontaire du Centre Canadien d'Etudes et de Coopération Internationale (C.E.C.I.) à titre de conseiller en planification rattaché au Département de Planification Régionale (D.P.R.) de la région Bolama-Bijagos du Ministère du Plan de la Guinée-Bissau.

Nous tenons à remercier les différentes personnes et organismes qui ont rendu cet exercice réalisable, soit: les autorités nationales (Ministère du Plan, Ministère de l'Agriculture et des Pêches) et régionales (Comité d'Etat de la Région Bolama-Bijagos, D.P.R.-Bolama), les organismes de coopération internationale (C.E.C.I., Iles de Paix, Pescarte), particulièrement ma conjointe Brigitte, et l'équipe de Guinée-Bissau et de Montréal du Projet de Développement Agricole Bolama-Bijagos du C.E.C.I.. Une attention toute spéciale doit être portée aux principaux intéressés, les habitants de l'île de Bolama et, par extension, de la région toute entière. 


\section{TABLE DES MATIERES}

page

RESUME

ii

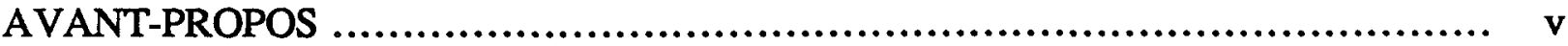

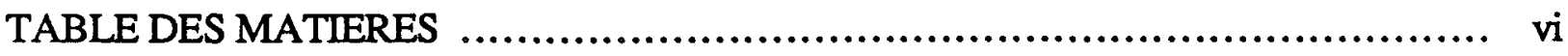

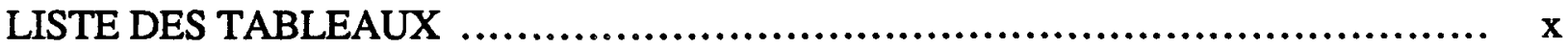

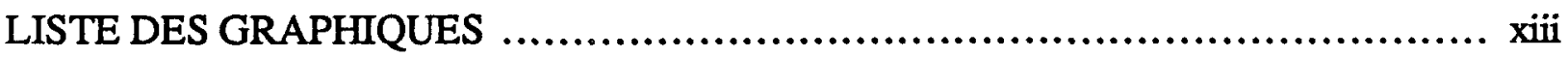

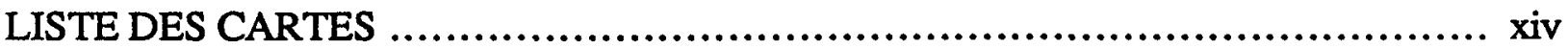

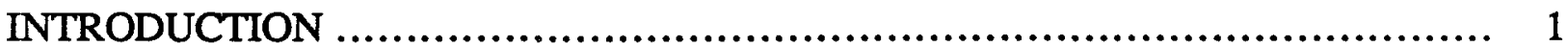

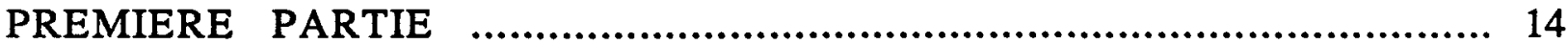

1. LA GUINEE-BISSAU ET LA REGION BOLAMA-BIJAGOS $\ldots \ldots \ldots \ldots \ldots \ldots \ldots \ldots . . . . . .16$

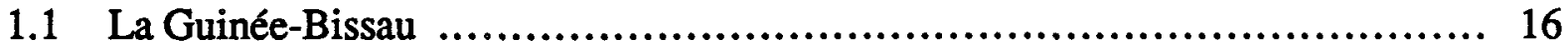

1.1.1 Géographie et climat ................................................. 16

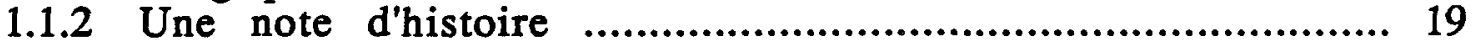

1.1.3 Le pays aujourd'hui ................................................ 25

1.1.3.1 Modes de production ...................................... 29

1.1.3.2 Importations et exportations .............................. 38

1.2 La région Bolama-Bijagos ............................................. 51

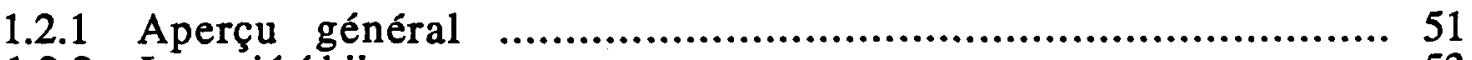

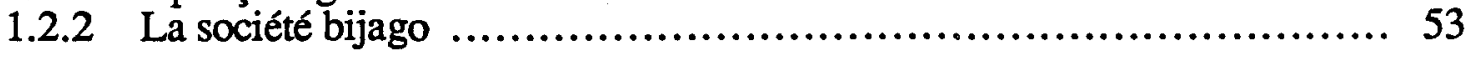


2. L'ILE DE BOLAMA, UNE REGION EN SOI ............................... 59

2.1 Description physique ......................................................... 59

2.2 Bolama, d'hier à aujourd'hui ........................................... 67

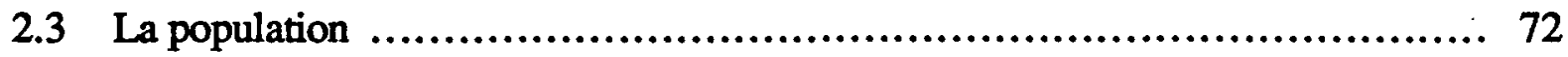

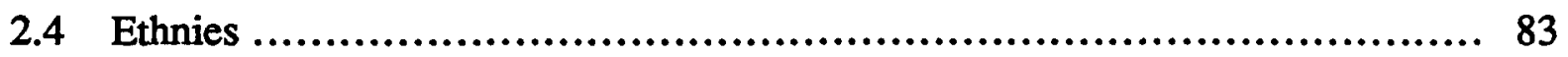

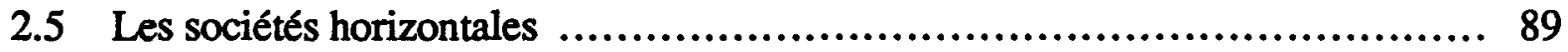

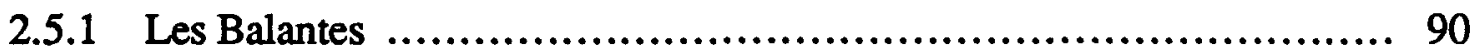

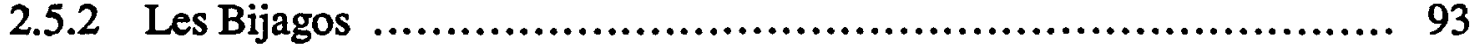

2.6 Les sociétés verticales ............................................................ 96

2.6.1 Les Manjaques, Pepels et Brâmes/Mancagnes .......................... 96

2.6.2 Les Foulas, Mandingues et Beafadas ................................. 99

3. LE MILIEU SOCIO-ECONOMIQUE BOLAMIEN ............................... 104

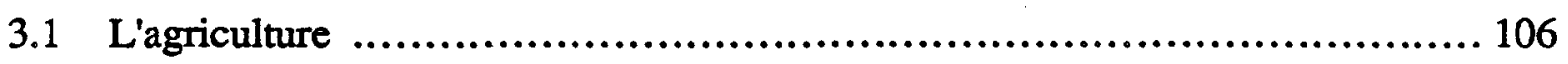

3.1.1 Unité familiale d'exploitation .......................................... 109

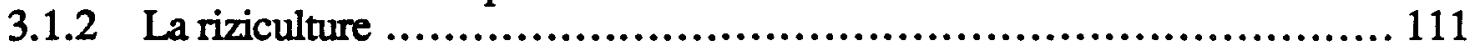

3.1.3 Le maraîchage .................................................................. 114

3.1.4 Les fermes d'Etat ................................................................ 120

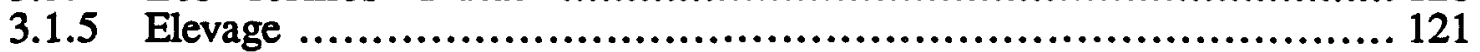

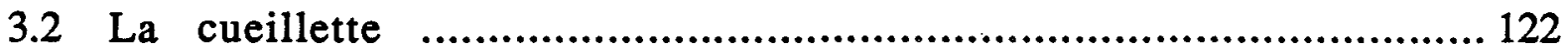

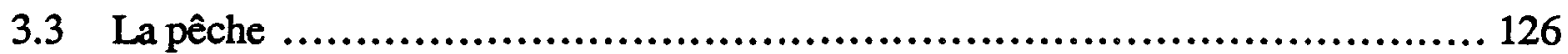

3.3.1 La pêche artisanale ......................................................... 126

3.3.2 La pêche professionnelle .................................................... 129

3.4 Les activités artisanales .................................................... 133

3.4.1 Spécialisations ethniques ................................................... 138

3.4.2 Division sexuelle du travail ....................................................... 139

3.4.3 Répartition saisonnière des activités ..................................... 144 


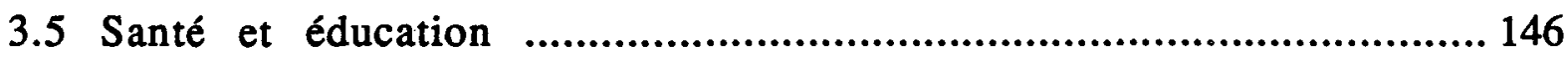

3.5.1 Santé .................................................................. 146

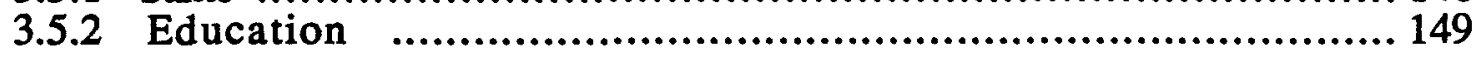

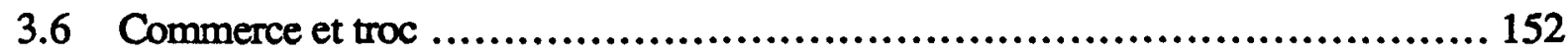

3.7 Autres activités économiques ............................................. 163

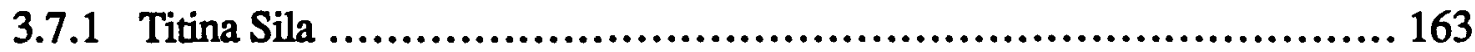

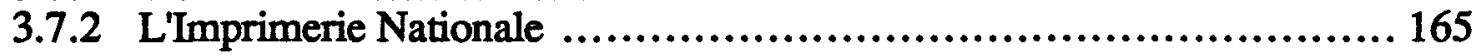

3.8 Les projets de coopération internationale .................................. 165

3.8.1 D.P.R.-Bolama-Bijagos .......................................... 166

3.8.2 Le Projet de Développement Agricole Bolama-Bijagos ................. 168

3.8.3 Le projet de pêche Pescarte/Ittica Valdagri ............................. 172

3.8.4 Ile de Paix ...................................................... 175

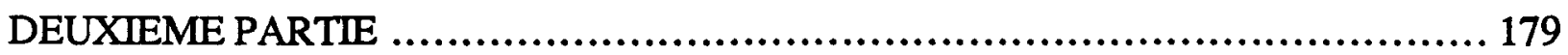

4. LES STRUCTURES POLITICO-ADMINISTRATIVES .......................... 180

4.1 Le P.A.I.G.C. ..................................................... 180

4.2 Structures politiques et administratives .................................... 184

4.2.1 Les comités de tabanca ............................................... 184

4.2.2 Le pouvoir régional ..................................................... 189

4.2.3. Le pouvoir administratif ................................................... 190

4.2.4 Le pouvoir national ................................................. 192

4.2.5 Les provinces .................................................. 194

5. LE MODELE DE DEVELOPPEMENT BISSAU-GUINEEN ...................... 200

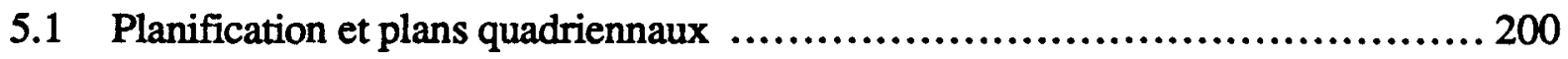

5.1.1 Le Premier Plan Quadriennal de Développement Economique et Social, 1983-1986 ................................................................ 203

5.1.2 Le Deuxième Plan Quadriennal de Développement Economique et Social ................................................................ 222

5.1.2.1 Prévisions .............................................................. 222

5.1.2.2 La production populaire …................................... 228

5.2 Les structures régionales de planification ............................................ 232

5.2.1 La Division de Planification Régionale (D.P.R.) ........................... 233

5.2.2 Les Cabinets Régionaux de Planification (G.R.P.) ..................... 238

5.3 SOLIDAMI, création d'une O.N.G. bissau-guinéenne .............................. 242 
6. POPULATION LOCALE ET DEVELOPPEMENT PLANIFIE 246

6.1 Les aspirations locales .................................................... 248

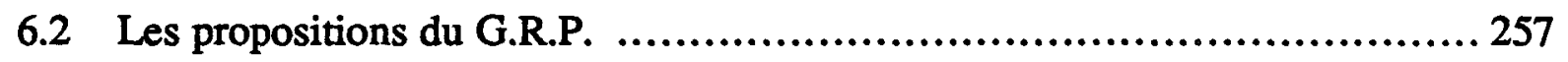

6.3 Les orientations de projet ............................................ 266

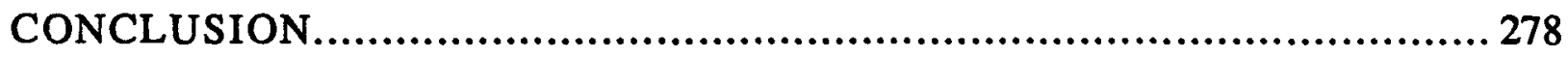

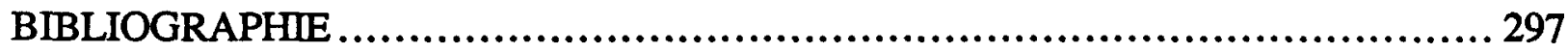

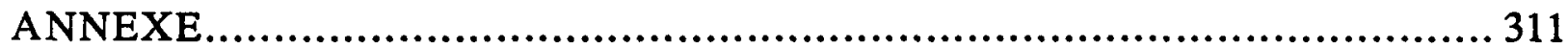




\section{LISTE DES TABLEAUX}

page

TABLEAU 1.1 Principales données démographiques, Guinée-Bissau

TABLEAU 1.2 Distribution de la population totale du pays selon l'âge, Guinée-Bissau, 1986

TABLEAU 1.3 Quelques données démographiques comparatives, Guinée-Bissau/monde

TABLEAU 1.4 Distribution et estimation de la population active et salariée selon le secteur d'activité Guinée-Bissau, 1979-1980

TABLEAU 1.5 Balance commerciale 1981-1985, Guinée-Bissau (en millions de dollars américains)

TABLEAU 1.6 Balance commerciale, Importations et exportations annuelles, Guinée-Bissau, 1960 à 1981 (en millions de contos)

TABLEAU 1.7 Structure des exportations, Guinée-Bissau, - 1983-1985 (en \% du total)

TABLEAU $1.8 \quad$ Nature des exportations, Guinée-Bissau, 1977 (en \% de la valeur totale)

TABLEAU 1.9 Structures des importations, 1983-1985, Guinée-Bissau, (en \% de la valeur totale)

TABLEAU 1.10 Disponibilité totale de riz, Importations et production nationale, 1977-1985, Guinée-Bissau (en tonnes)

$\begin{array}{lll}\text { TABLEAU 2.1 } & \text { Principales données climatiques, Me de Bolama }\end{array}$

TABLEAU 2.2 Pluviométrie annuelle moyenne, Ile de Bolama, 1924-1987 62 
TABLEAU 2.3 Occupation des sols, Ile de Bolama, 1978 (en hectares) 65

TABLEAU 2.4 Population totale, Me de Bolama, 1979 et $1987 \quad 76$

TABLEAU 2.5 Distribution de la population selon l'âge et le sexe, Ile de Bolama, 1987

TABLEAU 2.6 Distribution ethnique, Région Bolama-Bijagos et ensemble du pays, avril 1979 (en \%)

TABLEAU 2.7 Ethnies principales et secondaires selon le village, Ile de Bolama, 1987

TABLEAU 3.1 Calendrier des principales activités, Région Bolama-Bijagos 107

TABLEAU 3.2 Calendrier des activités maraîchères, lle de Bolama 116

TABLEAU 3.3 Répartition des tâches maraîchères, selon l'âge et le sexe, lle de Bolama

TABLEAU 3.4 Nombre de pêcheurs et de canots, Ile de Bolama, 1987

TABLEAU 3.5 Tableau de frequence des activités de production selon le nombre de villages, Ile de Bolama, 1987

TABLEAU 3.6 Répartition des principales tâches agricoles selon l'âge et le sexe, Ile de Bolama

$\begin{array}{lll}\text { TABLEAU } 4.1 & \text { Structures politico-administratives, Guinée-Bissau } & 188\end{array}$

TABLEAU 4.2 Province du Sud, Division administrative, 1987

TABLEAU 5.1 Premier Plan Quadriennal, dépenses de développement, 1983-1986 (en millions de P.G.)

TABLEAU 5.2 Distribution des investissements, Premier Plan Quadriennal, Région Bolama-Bijagos et ensemble du pays, 1983-1986, (en millions de P.G.)

TABLEAU 5.3 Répartition des sommes consacrées au Premier Plan Quadriennal, Région Bolama-Bijagos,1983-1986 (en millions de contos)

TABLEAU 5.4 Taux de réalisation des investissements par secteurs pour le Premier Plan Quadriennal, 1983-1986 (en millions de P.G.) (prix constants de 1983)

TABLEAU 6.1 Tableau de fréquence des principales demandes de développement selon le nombre de villages, Enquête de tabanca, lle de Bolama, 1987 
TABLEAU 6.2 Répartition des principales demandes selon le village, Enquête de tabanca, Ile de Bolama, 1987

TABLEAU 6.3 Causes d'abandon des rizières par village, Ile de Bolama, $1987 \quad 255$

TABLEAU 6.4 Liste des propositions de projets pour 1986, G.R.P.-BolamaBijagos

TABLEAU 6.5 Liste des propositions de projets pour 1987, G.R.P.-BolamaBijagos

TABLEAU 6.6 Liste des projets prioritaires pour 1988, G.R.P.-Bolama-Bijagos

TABLEAU 6.7 Liste des projets prioritaires pour le Second Plan Quadriennal, Région Bolama-Bijagos 


\section{LISTE DES GRAPHIQUES}

page

GRAPHIQUE I Importations et exportations annuelles, Guinée-Bissau, 1960-1981 (en millions de contos par année)

GRAPHIQUE II Pluviométrie annuelle moyenne, Ile de Bolama, 1924-1987

GRAPHIQUE III Distribution de la population selon le sexe et l'âge, lle de Bolama, 1987 


\section{LISTE DES CARTES}

page

CARTE 1

Guinée-Bissau

15

CARTE II

La région administrative Bolama-Bijagos

50

CARTE III

Villages enquêtés, Ile de Bolama

58 
"Comme il arrive avec la fleur dans une plante, c'est dans la culture que réside la capacité (ou la responsabilité) d'élaboration du germe qui assure la continuité de l'histoire en assurant en même temps la perspective de l'évolution et du progrès de la société en question."

(Amilcar Cabral) 1

${ }^{1}$ Mario de Andrade, Amilcar Cabral: essai de biographie politique Paris, Librairie François Maspero, 1980, page 154. 


\section{INTRODUCTION}

Dans la lignée des guerres de libération nationale des années 1960 et 1970, la GuinéeBissau, ex-Guinée Portugaise, occupe avec son leader Amilcar Cabral, son peuple et son parti, une place de choix, tant au niveau de l'originalité de son modèle d'émancipation que de la sympathie que cette lutte lui a valu sur le plan international. ${ }^{1}$ L'accord d'Alger du 24 août 1974 entre le P.A.I.G.C. et le Portugal confirme "la reconnaissance de la République de Guinée-Bissau" et le "droit du peuple du Cap-Vert à l'indépendance",2 chose que la pratique révolutionnaire avait rendue effective, du moins dans les zones libérées, comme l'atteste la proclamation unilatérale le 24 septembre $1973{ }^{3}$ par l'Assemblée Nationale Populaire, de l'indépendance et de la souveraineté du pays .

De nos jours, la Guinée-Bissau figure parmi les pays les moins développés du monde avec un P.I.B. en 1988 de 179 \$ millions américains (soit un P.I.B. per capita de 190 \$ américains) et une espérance de vie à la naissance, toujours en 1988, de 45 ans. ${ }^{4}$ Très replié

1 Outre le lien très étroit qui a su être tissé entre les forces de libération du P.A.I.G.C. (Parti Africain pour l'Indépendance de la Guinée et du Cap-Vert) et les masses paysannes, l'organisation des territoires libérés constitue un modèle original et bien adapté au milieu. Bien que l'objet du mémoire ne porte pas sur la période de libération, nous ne pouvons taire cet état de fait; le premier chapitre exposera les principales lignes de cette période qui marqua ce petit pays.

2 Paulette Pierson-Mathy, La naissance de lEtat par la guerre de libération nationale: le cas de la GuinéeBissau, Paris, U.N.E.S.C.O., 1980, 155 pages.

3 Ibid. page 23.

4 L'État du monde, édition 1991, annuaire économique et géopolitique mondial. Éditions La Découverte, Éditions du Boréal, Montréal, 1990. Page 255. 
sur lui-même, ce pays de 780985 habitants selon le recensement de 19791 , est victime, à l'instar de nombreux autres pays du tiers-monde, d'une capitale hypertrophiée qui monopolise toutes les ressources du pays et de l'aide internationale. ${ }^{2}$

Une coupure très nette entre la campagne et la ville est facilement perceptible. Elle se réflète particulièrement dans la différence entre les modes de vie urbains et ruraux. En ville, on note la présence du salariat (plus ou moins important cependant), ${ }^{3}$ alors qu'à la campagne, le repli quasi-autarcique des populations sur elles-mêmes et l'absence de flux d'échange entre la campagne et la ville sont facilement perceptibles. ${ }^{4}$

Le pays ayant donc un visage nettement rural, toute étude sera influencée par la prédominance d'une économie de subsistance. Toute tentative d'intervention devra impérativement en tenir compte.

1 Ministério da Coordenação Economica e Plano, Recenseamento Geral da Populacãoe Habitacão. 16 de abril de 1979: resultados provisorios (fase II), Departamento Central de Recenseamento, Bissau, fevereiro 1982, 241 pages + annexes.

2 Bissau a connu ces dernières années un boom démographique considérable passant de 18325 habitants en 1950 à 109214 en 1979 (S.E.P.C.I., Primeiro Plano Ouadrienal de Desenvolvimento Economico e Social 1983-1986. Volume 1. Relatorio Geral, Bissau, page 50) et 160000 en 1985 (SCET-AGRI, Etude des prix et incitations aux producteurs ruraux MICEPCI, République de Guinée-Bissau, janvier 1986, page I).

Carlos Lopes souligne qu'alors qu'elle ne compte qu'environ $16 \%$ de la population totale du pays, Bissau monopolise $50 \%$ des investissements et $80 \%$ du budget de fonctionnement normal (Carlos Lopes, "Ethnie, Etat et rapports de pouvoir en Guinée-Bissau", Itinéraires. notes et travaux, No. 22, mars 1982, Première Edition, Genève, page 75).

3 Amilcar Cabral, Unité et lutte, Paris, Petite Collection Maspero, 1980, page 51.

4 Toujours selon Lopes, 56\% de l'économie, en se basant sur le PIB de 1977, se situerait dans le secteur de l'auto-subsistance, soit des rapports non-marchands (Carlos Lopes,, op. cit., page 31). Certains auteurs vont même plus loin: "Plus de $80 \%$ de la population de Guinée-Bissau vit de l'agriculture, qui est l'activité dominante du pays.

L'agriculture est caractérisée par une production qui vise à satisfaire les propres besoins (consommation en produits de première nécessité) des agriculteurs. Ceux-ci sont très peu motivés par une production excédentaire dont ils ne peuvent tirer profit" (SCET-AGRI, op. cit., page 17). 
Economie de subsistance

Comme le souligne Robert Badouin, l'économie de subsistance a comme base l'agriculture, ${ }^{1}$ une agriculture dégageant peu de surplus, si ce n'est pour parer aux éventuelles calamités naturelles. Cette agriculture se limite à des cellules étroites, la famille, le clan, la tribu, le village ou le campement. ${ }^{2}$ Dans l'économie de subsistance, on note:

..."la primauté de l'activité agricole pour un groupe de faible dimension, menant une vie autarcique et percevant sa continuité comme dépendante du respect de la tradition et de la quête des subsistances." 3

De même ajoute-t-on plus loin que,

"L'agriculture de subsistance désigne ceux des systèmes économiques qui bornent l'ambition des membres d'un groupe de dimensions modestes à l'obtention des seuls biens et services pouvant être proposés à l'intérieur du groupe. Dans de telles conditions, la production de biens sera limitée à la couverture des besoins les plus urgents et ne dépassera guère un niveau correspondant à la stricte satisfaction des besoins d'ordre physiologique." 4

On comprendra donc que, sauf lorsqu'il y a une forte pression démographique, le travail de la terre n'est pas intensif et, habituellement, l'homme demeure oisif une bonne partie de l'année. 5 De même, les instruments de travail sont assez rudimentaires et l'on ignore, en théorie, les cultures attelée et motorisée. ${ }^{6}$

L'adoption d'un modèle d'économie de subsistance par une population sera à prendre en considération par quiconque voudra intervenir sur le milieu. L'augmentation de la productivité n'apparaîtra pas nécessairement chez les populations concernées comme

1 Robert Badouin, Economie rumale, Paris, Librairie Armand Colin, 1971, page 19.

2 Ibid., page 20.

3 Ibid., page 19.

4 Ibidem.

5 Ibid., page 25.

6 Ibid., page 26. 
nécessaire, ainsi que les changements dans les méthodes ou les types de cultures, ou encore l'abandon de la technique de culture dominante. En effet, comme le souligne Badouin, "D'une manière générale, il semble que l'économie de subsistance ne cherche pas à assurer à ses membres le meilleur régime alimentaire possible, mais la manière la plus facile de pourvoir à leur besoin de nourriture."

"Dès lors il n'est pas certain qu'un changement dans le système de production, le passage de la culture manuelle à la culture attelée, par exemple, aboutisse à une élévation de la productivité. Si le cultivateur doit renoncer à un temps de loisir assez long pour obtenir un supplément de revenu assez maigre, il sera peu séduit par l'opération. Un système de production techniquement supérieur peut ne pas le tenter, car le revenu attaché au temps additionnel de travail lui paraît trop réduit." 1

C'est dans ce contexte que nous nous proposons d'effectuer un travail de description et d'analyse portant sur une île, Bolama, d'une région insulaire à caractère rural dominant. La région Bolama-Bijagos, qui est composée de 87 îles dont une vingtaine est habitée, constitue certes l'une des régions les plus désavantagées et isolées du pays. Des problèmes de pénurie quasi-constante des biens de première nécessité (pétrole, denrées alimentaires, médicaments), la précarité des services gouvernementaux et l'absence de transport adéquat en font une région défavorisée, dont les meilleurs éléments du pays en matière de ressources humaines ne font que fuir. Ainsi cette région n'est-elle souvent qu'une "retraite" bien tranquille pour les fonctionnaires les plus déclassés. Dépourvue de richesses naturelles importantes, ses principaux atouts (tourisme, huile de palme, pêche) sont peu ou mal exploités et elle se retrouve donc le plus souvent laissée à elle-même.

${ }^{1}$ Ibid., page 27. 
Région et développement

Un premier examen de la carte II (voir à la page 50) nous indique que la région BolamaBijagos constitue un ensemble apparemment homogène. La seule ombre au tableau demeure la présence d'une partie du continent au sein de cette région administrative, la presqu'île de São-João, au nord-est, qui contraste avec l'insularité du reste de la région. Cependant, certaines disparités rendent l'homogénéité régionale encore plus ambiguë. Ainsi, la structure ethnique régionale, les flux de personnes et les traditions tendent à isoler l'île de Bolama et São-João du reste de l'archipel. Comme nous le verrons ultérieurement, la population de l'archipel bijago est massivement orientée vers lîle de Bubaque, Bolama restant marginale et isolée par rapport au reste de la région. En ce sens, Bolama, à l'exception de quelques villages habités par l'ethnie bijago, est plus près du continent que du reste de l'archipel. Nous abandonnerons donc pour les fins de notre ouvrage la délimitation bissau-guinéenne de la région administrative Bolama-Bijagos, pour concentrer nos efforts sur l'île de Bolama qui, en soi, correspond relativement à notre conception de la région:

"La région correspond à une aire géographique constituant une entité qui permette, à la fois, la description des phénomènes naturels et humains, l'analyse des données socio-économiques et l'application d'une politique. Elle se fonde sur deux caractères principaux: homogénéité et intégration fonctionnelle et s'achève, à la fois, par le sentiment d'une solidarité vécue et par des relations d'interdépendance avec les autres ensembles régionaux et avec l'espace national et international." 1

Qui plus est, lîle de Bolama, contrairement au reste de l'archipel n'est pas peuplée uniquement de Bijagos mais présente une richesse ethnique évidente. Une enquête effectuée auprès de 25 communautés villageoises de l'île nous a permis d'identifier la présence de plusieurs ethnies différentes (Bijagos, Mancagnes, Manjaques, Balantes, Foulas,

1 Pierre Delfaud, Claude Lacour, Joseph Lajugie, Espace régional et aménagement du territoire, Paris, Précis Dalloz, 1979, page 143. 
Nhomincas, Beafadas, Mandingues et Pepels). Chacune de ces ethnies a ses particularités propres, son mode de vie, ses aspirations et sa culture. C'est dans ce contexte que notre travail de réflexion cherchera, à partir des particularités socio-culturelles de ces communautés, à envisager, dans la mesure du possible, des pistes à suivre en matière de développement. $\mathrm{Vu}$ l'inexistence d'un minimum d'infrastructures économiques à Bolama, la marginalisation du salariat et la persistance de l'économie de subsistance, nous ne saurions envisager de pistes de développement inspirés du modèle occidental. De ce fait, toute proposition quantitative en matière de croissance économique nous semblera, pour l'instant du moins, inadaptée à la réalité bolamienne, et nous opterons plutôt pour une conception plus "modeste" du développement, l'identifiant à la capacité d'une société de se prendre en main:

"Le développement n'est pas la position d'une société sur une ligne d'évolution. Il est dangereux de définir une société comme développée ou sousdéveloppée. Le développement est l'ensemble des actions qui fait passer une collectivité d'un type de société à un autre, défini par un degré plus élevé d'intervention de la société sur elle-même." 1

C'est donc en rejetant la primauté de l'économique et de ses projets spectaculaires qui, bien souvent, ne touchent qu'une minorité ou des intérêts extra-régionaux, que nous allons opter pour une philosophie de développement au "ras du sol", pour employer une expression de G. Sicault qui définit ce modèle de développement comme:

..."la prise en charge, partielle ou totale, par les communautés ou les groupements de communautés, de responsabilités et d'actions directes correspondant à leurs besoins propres, aux priorités qu'elles apprennent à établir en prenant progressivement conscience des problèmes qui affectent leur niveau de vie et leur bien-être. Cette forme de développement est rendue possible par un effort d'organisation, par l'utilisation d'un personnel choisi par la communauté, formé et recyclé sur place, et devenant partie intégrante et moteur de développement." 2

1 Alain Touraine, Les sociétés dépendantes: essais sur l'Amérique Latine, Paris, Duculot, 1976, page 9.

2 Dr G. Sicault, "Le développement "au ras du sol"', Revue Tiers-Monde, Tome XVIII, no. 72, octobredécembre 1977, page 705. 
Notons que nos ambitions sont très modestes, l'objectif du mémoire n'étant pas de proposer un modèle mais plutôt de rechercher des pistes possibles ou encore de définir un certain modèle de développement. Tout au plus aspirons-nous, dans une certaine mesure, et compte tenu des spécialisations et des ambitions de chaque ethnie, à ce que le lecteur puisse dégager certaines généralisations pour le reste de l'archipel, généralisations qui trouvent leurs limites dans les particularités de chacune des îles qui, vu leur isolement, constituent chacune des microcosmes en elles-mêmes. De même, nos intentions ne sont pas non plus de dresser une liste de projets à financer ou encore d'activités à effectuer. En fait, nous jugeons qu'un tel travail correspond plus au cadre et à la logique animant une mission de formulation de projet qu'à celui d'un mémoire universitaire auquel nous voulons conférer un caractère plus général et aussi moins dépendant des facteurs temporels faisant des projets d'aujourd'hui, des réalités, des échecs, des succès ou des oubliés de demain. Cette approche, la seule qui puisse d'après nous conserver une valeur consultative et informative sur la réalité bolamienne de la fin des années 1980, ne peut permettre au lecteur recherchant des mesures concrètes à mettre de l'avant, de trouver réponse à ses interrogations en matière de développement d'une société sur de nombreux points traditionnelle. Le mémoire ne pourra non plus permettre à l'intervenant sur le terrain d'y trouver un plan de travail précis. En fait, nous aspirons plutôt à en faire un instrument de documentation et un outil de réflexion exposant les données d'un problème particulier de développement d'une région insulaire et isolée, dépourvue de richesse particulière, où vivent deux mondes sans relation entre eux, l'un replié sur lui-même et l'autre, plus moderne, caractérisé par le salariat, la non-possession par l'individu de ses moyens de production et sa dépendance face à l'aide externe, tant alimentaire que technique.

Puisque nous reconnaissons à chaque communauté la responsabilité d'intervention sur son devenir, nous croyons que, dans le cas particulier des communautés nécessitant une aide 
externe en la matière, cette aide doive être en accord avec les vues et aspirations de la communauté.

Dans le cas bolamien, l'intervention d'agents de développement s'effectue sous deux formes, celle des autorités gouvernementales bissau-guinéennes grâce aux différents ministères (Ministère du Développement Rural, Ministère du Plan, Ministère de la Santé, etc.) et organes en région (le Secrétariat d'Etat aux Pêches), et celle d'organismes de coopération internationale (C.E.C.I., Ile de Paix) et de firmes privées (Ittica Valdagri Spa). La pertinence du mémoire doit ainsi résider dans le dépassement de la simple description de la société bolamienne pour déboucher sur l'analyse de l'impact réel des activités de développement menées par ces agents, selon une grille d'évaluation basée sur la reconnaissance des particularités et des aspirations ethno-culturelles des communautés locales. Suivant notre grille d'analyse, nous comprenons que la dynamique qui saura ou non se créer entre les agents de développement et la population, de même que la persistance, la nature et l'envergure de cette même dynamique, pourront être considérés comme d'intéressants indicateurs pour évaluer la pertinence de l'intervention. D'ailleurs, nous croyons que l'appartenance des intervenants en développement à Bolama à des organisations différentes tant sous leur forme, leur origine que leur approche, garantit en quelque sorte une diversité de méthodes et de résultats. Nous pourrons ainsi mieux évaluer la portée de chaque intervention et, de là, nous assurer une meilleure compréhension de la culture locale.

Il faut convenir cependant de l'aspect particulier du milieu bolamien et bissau-guinéen, ce que ne saurait ignorer quiconque veut comprendre la réalité du milieu. Ainsi, la situation socio-économique de l'île est des plus précaire. L'île de Bolama se compose d'une quarantaine de petits villages appelés tabancas, plus ou moins engagés dans une économie de type autarcique où chacun (village) se suffit à lui-même, et d'un petit centre urbain de moins 
de 3000 habitants, Bolama. Tout comme au niveau national, nous assistons à une coupure rural/urbain où les échanges entre ces deux mondes sont pratiquement inexistants. Il en découle que la ville de Bolama connait de constants problèmes d'approvisionnement. Les villages pour leur part, engagés dans un procès autarcique, ne dépendent de l'extérieur que pour combler d'éventuels déficits vivriers en matière de riz qui sont plus ou moins fréquents selon les villages et les ethnies. Nous pouvons sans crainte avancer que l'île de Bolama est peu intégrée à l'économie de marché. A preuve, la persistance et la préférence des populations locales pour les échanges sur la base du troc et la rareté des biens à échanger ou vendre.

Rechercher la cause de cette situation (persistance d'un modèle d'échange basé sur le troc) déborderait le cadre de notre recherche. Il faudra cependant en tenir compte dans notre analyse et y voir non seulement, selon une logique économiste, un manque de confiance de la population face à une monnaie sur-évaluée et non-convertible, n'ayant pas grande valeur d'échange (suivant le cercle vicieux où l'on ne trouve rien sur le marché parce que les gens ne veulent rien proposer en échange de pesos guinéens, et où l'on n'a pas besoin d'argent puisqu'il n'y a rien à acheter), mais plutôt une certaine faillite de l'effort de construction nationale d'après-guerre, qu'elle soit due au manque de participation populaire ou, tout simplement, à l'adoption d'un modèle de développement inadéquat.

La stagnation que nous connaissons peut bien sûr trouver sa source dans les plaies qu'ont laissées de nombreuses années de guerre. Mais il ne s'agit sûrement pas là de la seule cause. De même, l'engagement quasi-général sur la base nationale de la population à la cause de la lutte armée confirme le fait que la population peut s'impliquer dans une cause commune. Alors qu'en est-il de la reconstruction nationale? Est-ce que, suite aux nombreux espoirs générés par le P.A.I.G.C., le peuple guinéen serait entré dans une phase attentiste 
provoquant peu à peu une démobilisation, démobilisation nettement perceptible dans le discours de nombreux citoyens?

Quoiqu'il en soit, bien que l'objet du mémoire ne soit pas d'étudier les aspects sociologiques de la lutte et de la reconstruction nationale proposée par le Parti, nous comprenons bien que nous devrons fréquemment déborder du cadre suggéré, tout ceci ne rendant le problème que plus complexe.

\section{Méthodologie}

Du point de vue méthodologique, notre séjour en terre bissau-guinéenne et le manque évident de ressources bibliographiques sur l'île nous forcent à nous orienter vers la collecte de données empiriques. Loin de constituer une entrave à notre réflexion, cette démarche sur le terrain, tout au contraire, aura permis un certain contact avec la réalité telle que vécue par la population. Le présent ouvrage concerne particulièrement les années passées sur le terrain (de 1986 à 1988) de même que celles précédant l'intervention. On comprendra que la période séparant la fin du séjour et le dépôt du mémoire (1988 à 1990) ne pourra être couverte, ce qui peut contribuer, sous certains aspects, à laisser le lecteur en quelque sorte sur sa faim.

Parmi les outils principaux de travail nous comptons l'enquête de village (dite "enquête de tabanca") effectuée dans 25 villages de l'île aux mois d'avril et mai 1987 par les membres de la Délégation Régionale du Plan. Originant du Ministère du Plan central de Bissau, cette enquête aura été quelque peu modifiée par l'équipe régionale de la D.P.R., notamment par l'ajout d'une série de questions sur les productions artisanales et les besoins particuliers du milieu. Les questionnaires (voir en Annexe) auront été soumis aux membres des comités de 
villages qui se montraient généralement très conscients des besoins de leur village. $\mathrm{Ce}$ questionnaire constitue certes l'un des outils fondamentaux de notre recherche puisqu'il nous aura permis de dresser une liste des principales propositions en matière de développement villageois, en plus de nous donner l'opportunité de nous "introduire" auprès des comités de village et d'ainsi initier une des processus fondamentaux de la culture africaine, si nécessaire et fondamental dans toute approche d'intervention, soit la palabre, l'échange humain.

De plus, nous avons coordonné deux recherches menées entre novembre 1987 et mai 1988 par l'équipe du Projet de Développement Agricole Bolama-Bijagos du C.E.C.I., portant sur la commercialisation des produits maraîchers et l'étude des systèmes de production. 1

Enfin, au niveau pratique, plusieurs entrevues faites auprès de personnes impliquées dans des projets de développement régionaux nous auront grandement aidés.

Du point de vue bibliographique, nous avons pu avoir accès à divers documents sur la Guinée-Bissau, son système de planification et les divers projets de développement en place, de même que sur la région de Bolama, au centre de documentation du D.P.R.-Bolama (Centro de Documentação Bolama-Bijagos, C.D.B.B.), l'I.N.E.P. (Instituto Nacional de Estudos e Pesquisa) de Bissau et aux centres de documentation des ministères du Plan et de l'Agriculture à Bissau.

C'est grâce à ces différents outils que nous nous proposons le travail suivant composé de deux parties, une première, descriptive, traitant du cadre bolamien et une seconde, plus dynamique, sur le modèle de développement régional bissau-guinéen. Nous croyons

1 Brigitte Boies, Alain Rousseau, Enquête sur la commercialisation des produits maraîchers, Projet de Développement Agricole Bolama-Bijagos, C.E.C.I., Bolama, mai 1988, 80 pages; et, Brigitte Boies, Alain Rousseau, Guide pour l'analyse des systèmes de production: archipel Bolama-Bijagos. Guinée-Bissau, Projet de Développement Agricole Bolama-Bijagos, C.E.C.I., Bolama, mai 1988, 26 pages + Annexes. 
nécessaire et fondamental d'arrimer notre conception du développement à une connaissance précise du milieu et de ses habitants. La première partie du mémoire s'y attaquera donc tout particulièrement. Ainsi, le premier chapitre se propose-t-il de décrire la Guinée-Bissau et la région Bolama-Bijagos tant du point de vue physique, qu'historique et social. Le second chapitre continue sur cette lancée mais en se penchant plus spécifiquement sur Bolama et la description des principales ethnies qui la peuplent, regroupées sous une analyse basée sur la structure du pouvoir. Cette démarche, nous menant du général au particulier, débouchera, au quatrième chapitre, sur la réalisation du portrait socio-économique de l'île. Les principales activités économiques de la population y seront traitées (agriculture, cueillette, pêche, artisanat, santé, éducation, commerce et troc) sous une lunette cherchant à dégager certaines particularités ethniques bien précises telles que les spécialisations ethniques, la division sexuelle du travail ou encore la répartition annuelle des activités de production. Ce chapitre ne saurait passer sous silence la présence des projets de coopération internationale actifs sur lî̀le.

La seconde partie constitue certes le noeud du mémoire visant à confronter théorie et pratique. Le lecteur qui aura pris connaissance des caractéristiques du milieu, pourra y trouver exposées, les différentes pratiques tant gouvernementales (chapitre cinq) qu'étrangères (chapitre six) en matière de développement. Ainsi, d'une manière plus précise, nous proposons-nous au quatrième chapitre de dépeindre les structures politicoadministratives du pays, ce qui s'avère à notre avis impératif à la compréhension du fonctionnement du modèle bissau-guinéen de planification du développement économique et social. Le modèle national de planification sera étudié au chapitre cinq sous ses différentes facettes, que ce soit par ses plans quadriennaux ou par les structures régionales de planification et le nouvel organe gouvernemental de coordination des activités de développement des O.N.G. (organisations non-gouvernementales) au pays, le Solidami. 
Tout ceci devra nous mener, au sixième chapitre, à confronter effectivement les aspirations locales et la pratique des intervenants en matière de développement socio-économique, soit essentiellement le gouvernement national et les projets de coopération. Nous serons de cette manière, espérons-nous, en mesure non seulement de décrire les interventions poursuivies, mais aussi d'analyser et d'évaluer la portée de ces actions en référence à notre propre théorie du développement. 
PREMIERE PARTIE 


\section{CARTE I}

GUINEE-BISSAU

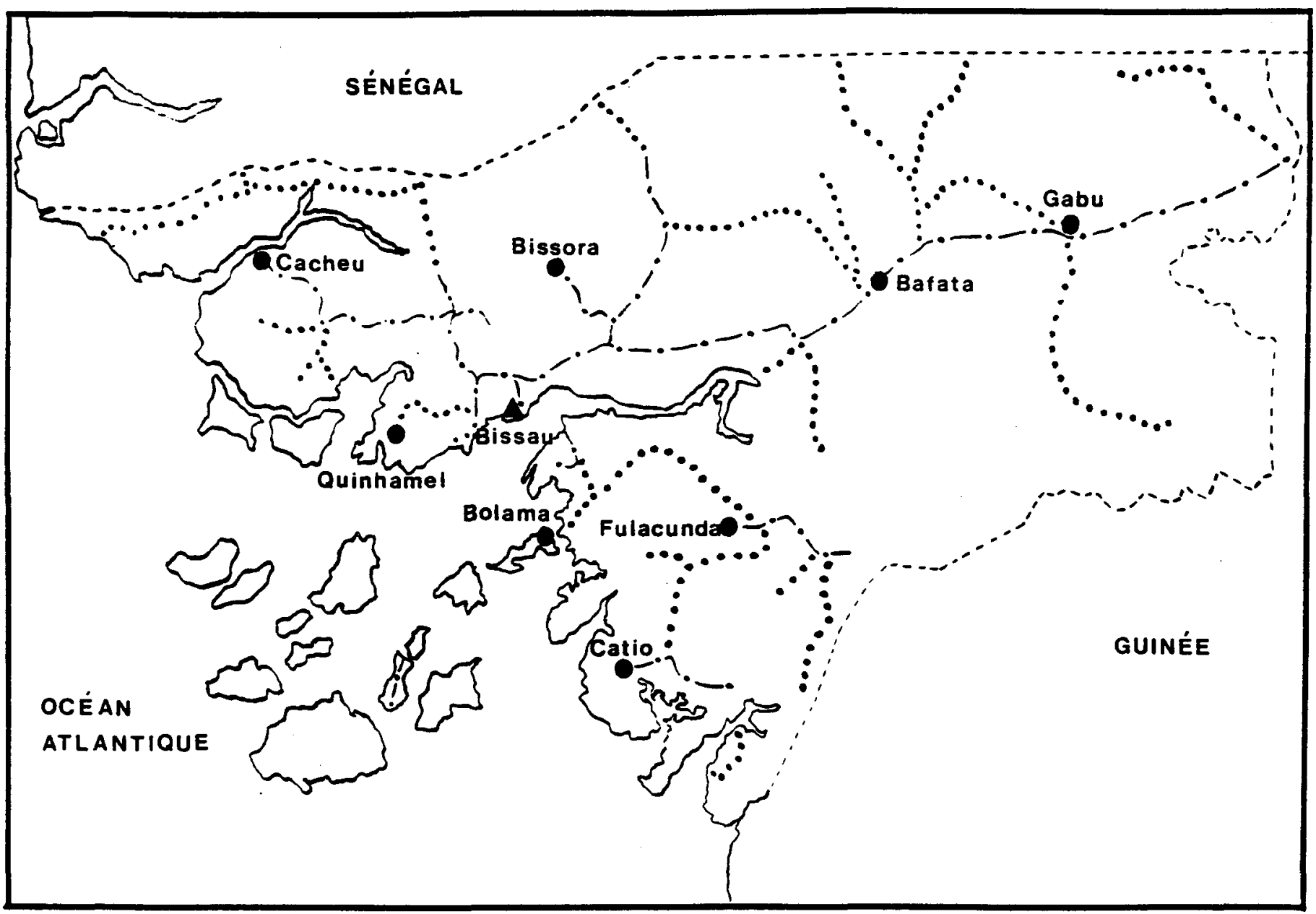

Capitale régionale

Capitale nationale

$01020304050 \mathrm{Km}$

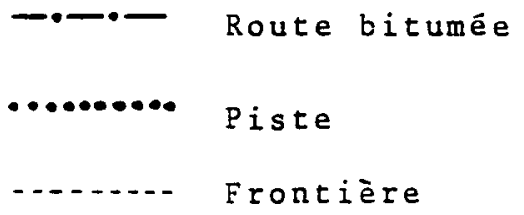

Source: Institut Géographique National-France. Guinée-Bissau. 1:50 000. Paris, 1981 . 


\section{CHAPITRE UN}

\section{LA GUINEE-BISSAU ET LA REGION BOLAMA-BIJAGOS}

\subsection{La Guinée-Bissau}

\subsubsection{Géographie et climat}

La Guinée-Bissau est un petit pays situé sur la côte occidentale de l'Afrique, entre le Sénégal et la République de Guinée (Guinée-Conakry). D'une superficie de $36125 \mathrm{~km}^{2}$, le pays s'étend entre $12^{\circ} 40^{\prime}$ et $10^{\circ} 57^{\prime}$ de latitude nord et entre $13^{\circ} 38^{\prime}$ et $10^{\circ} 49^{\prime}$ de longitude ouest. Le recensement de 1979 lui accordait une population de 777214 habitants, répartie en huit régions administratives (Biombo, Cacheu, Oio, Bafata, Gabu, Tombali, Quinara et Bolama-Bijagos), elles-mêmes subdivisées en 37 secteurs. Quant à la capitale Bissau, notons qu'elle jouit du statut de secteur autonome et est divisée en 37 quartiers. 1

1 Isabel Sarmento, Análise de sub-sistema de olaneamento e da administracăo regional da Republica da GuinéBissau, s.1., 1985, page 9. 
Sur le plan agricole, le pays est divisé en quatre zones: ${ }^{1}$

- Zone 1: Biombo (dont la capitale est Quinhamel), Cacheu (capitale: Cacheu), Oio (capitale: Bissora);

- Zone 2: Bafata (capitale: Bafata), Gabu (capitale:Gabu);

- Zone 3: Tombali (capitale: Catio), Quinara (capitale: Fulacunda); et,

- Zone 4: Bolama-Bijagos (capitale: Bolama).

Ces quatre zones ont été formées en 1978 pour les besoins de la planification régionale. Il avait alors été décidé de rassembler les huit régions administratives du pays pour former des zones "écologiquement homogènes", en accord avec la structure en matière de production agricole telle que définie par Amilcar Cabral lors de son enquête agricole de 1953.2

Du point de vue géographique, le pays se divise en deux grandes zones, les plaines maritimes et les plateaux de l'intérieur. ${ }^{3}$

Les plaines maritimes, qui occupent près de la moitié du territoire, sont très marécageuses et composées de nombreux fleuves et rivières, les "rios", dont l'effet de la marée pousse l'eau salée très loin à l'intérieur des terres, ce qui entraîne certaines conséquences néfastes pour l'agriculture. ${ }^{4} \mathrm{La}$ côte y est fortement découpée, le relief plat et les marées très fortes (jusqu'à sept mètres de hauteur).

1 Anne-Marie Hochet, Paysanneries en attente: Guinée-Bissau. Dakar, Environnement Africain, Série Etudes et Recherches, No. 79-80, janvier-février 1983, pages 3-8.

2 Yves Brunet, Nancy Thede, Rapport de mission d'étude sur le projet d'étude pour la mise en place de structures régionales de développement (Guinée-Bissau). Montréal, 5-7-79, page 3.

3 C.E.C.I.Guinée-Bissau, dossier pays.CECI, s.l., Programme de formation, décembre 1984, pages 4-6.

4 Anne-Marie Hochet, op.cit. page 6. 
Pour leur part, les plateaux de l'intérieur du pays sont peu élevés, atteignant difficilement les 300 mètres à la frontière de la Guinće-Conakry . 1

Le climat est de deux types, selon que l'on soit dans la région des plaines maritimes ou celle des plateaux de l'intérieur. Le long du littoral, le climat, appelé sous-guinéen, est chaud et humide, la température étant élevée et variant peu durant l'année. ${ }^{2} \mathrm{~A}$ titre d'exemple, la température moyenne annuelle de Bolama (de 1960 à 1974) est de $26,9^{\circ} \mathrm{C}$ et oscille entre $25,6^{\circ} \mathrm{C}$ en janvier et $28,2^{\circ} \mathrm{C}$ en mai. ${ }^{3} \mathrm{La}$ pluviométrie est très élevée, variant entre $1500 \mathrm{~mm}$ par an au nord et $3000 \mathrm{~mm}$ au sud. La saison des pluies s'étale de mai à novembre, alors que durant la saison sèche, les pluies sont rares, et l'humidité de l'air reste toujours très élevée. ${ }^{4}$ Ce climat, quoique très pénible pour l'homme, s'avère propice à la végétation. Du point de vue agricole, comme le note Anne-Marie Hochet, 5 il faut se garder cependant de sur-estimer l'effet favorable d'une pluviométrie élevée, une forte érosion en constituant l'envers de la médaille.

Le climat des plateaux de l'intérieur est, pour sa part, de type soudanien avec des températures légèrement supérieures à celles du littoral, et des variations fortes durant l'année $\left(24,3^{\circ} \mathrm{C}\right.$ en janvier contre $30,1^{\circ} \mathrm{C}$ en mai). La pluviométrie y est quelque peu inférieure (entre 1250 et $2000 \mathrm{~mm}$ par an du nord au sud) et la saison sèche, nettement moins humide au fur et à mesure que l'on avance vers l'intérieur des terres. ${ }^{6}$

1 C.E.C.I., op.cit., pages 5 -6.

2 Ibid. page 5.

3 Republica da Guiné-Bissau, Potentialités agricoles, forestières et pastorales, s.l., SCET-International, 1978, Volume I, page 92.

4 C.E.C.I., on cit., page 5.

5 Anne-Marie Hochet, op. cit., page 8.

6 C.E.C.I., op. cit, page 6. 
La Guinée-Bissau, au niveau physique, offre donc un caractère dual où se distinguent nettement une région littorale, au relief plat et à climat humide, et un arrière pays plus "montagneux", à climat nettement plus sec. Nous verrons comment, dans un chapitre ultérieur, cette dualité physique se retrouve aussi chez les habitants du pays qui, dans les régions côtières sont animistes et possèdent une toute autre culture que les ethnies musulmanes de l'intérieur des terres.

\subsubsection{Une note d'histoire}

C'est vers le milieu du $\mathrm{XV}^{\circ}$ siècle, probablement en 1446, que les Portugais accostèrent en Guinée-Bissau. ${ }^{1}$ Ce petit pays, sans grande richesse apparente et peuplé de nombreuses ethnies, n'intéressa les Portugais qu'aux $\mathrm{XVIII}^{\circ}$ et $\mathrm{XIX}^{\circ}$ siècles, comme comptoir commercial servant largement à la traite des esclaves. Grâce à l'appui de l'Angleterre, le Portugal hérita officiellement de la Guinée-Bissau à la Conférence de Berlin en 1844-1845. Le territoire était alors administré depuis les îles du Cap-Vert et ce n'est qu'à partir de 1870 que cette colonie fut détachée administrativement du Cap-Vert. Bolama devint alors la capitale de la Guinée Portugaise jusqu'en 1941, au profit de Bissau. ${ }^{2}$ Le territoire reçut en 1955, le statut de Province Portugaise d'Outre-Mer. ${ }^{3}$

La colonie guinéenne a été administrée d'une façon rétrograde et raciste, par une métropole qui négligeait de la mettre en valeur, chose qui n'est certes pas étrangère à l'apparition et au développement d'une prise de conscience politique radicale de la part de sa

1 Lars Rudebeck,Guinea-Bissau, A Study of Political Mobilization, Uppsala, The Scandinavian Institute of African Studies, 1974, page 3.

2 H. J. Dianoux, "La Guinée-Bissau et les îles du Cap-Vert",Afrique Contemporaine, No. 107, janvier-février 1976, pages 1-2.

3 J. R. de Benoit, "La Guinée-Bissau: le démarrage du nouvel Etat", Afrique Contemporaine, No. 83, janvierfévrier 1976, page 12. 
population. ${ }^{1}$ Citant Amilcar Cabral, Carlos Lopes analyse ainsi le colonialisme portugais en Guinée-Bissau:

"'En fait, le Portugal est seulement le gardien, quelques fois envieux des ressources humaines et matérielles de nos pays, au service de l'impérialisme mondial. Cela est la vrai raison de la survie du colonialisme portugais en Afrique et du possible prolongement de notre lutte. Cependant, plus que la présence de n'importe quelle puissance en Afrique, celle du Portugal a été et continue toujours à être dépendante de la présence d'autres puissances coloniales, surtout de l'Angleterre."

Partant de cette analyse de Cabral, nous pouvons bien situer le caractère du colonialisme portugais. Une nation relativement pauvre, sans ressource de grande valeur économique, avec une industrie faible qui ne permettait pas une accumulation forte de capital, ne pouvait pas, logiquement, entretenir des colonies en Afrique. Ce caractère de dépendance politique explique un certain nombre de particularités en Guinée:

Les infrastructures étaient catastrophiques: pas de routes, des télécommunications défectueuses même dans la ville de Bissau qui comptait 20000 habitants en 1950 et qui était la plus grande concentration urbaine. Pas d'écoles à l'intérieur du pays, et, celles de Bissau étaient réservées à la population assimilée (culturellement bien sûr!) qui se montait à quelques milliers en comptant les métis. L'assistance sanitaire se réduisait à une dizaine de postes sanitaires dans les principaux entrepôts commerciaux.

L'économie se limitait au commerce profitant à une petite bourgeoisie portugaise et aux grands trusts "Gouveia" appartenant au groupe portugais CUF et à la Sociedade Comercial Ultramarina. Ces sociétés s'occupaient surtout de l'achat des produits agricoles comme l'arachide, pour l'exportation. L'industrie n'existait pratiquement pas et les investissements portugais n'ont jamais été dirigés vers la Guinée-Bissau.

La culture européenne, chrétienne-occidentale, essayait de pénétrer une population, par les missionnaires, selon les principes de la politique d'assimilation. Les conditions pour obtenir une carte d'identité étant de savoir lire et écrire le portugais et se comporter comme un "civilisé". Le créole, langue véhiculaire du peuple guinéen et capeverdien, était interdit dans les écoles et méprisé par les Portugais. Les traditions culturelles de ce peuple étaient considérées comme des manifestations "sauvages".

La stagnation économique du pays provoquait de graves problèmes car les portugais se montraient vraiment rétrogrades dans leurs méthodes, fruit de leur

1 Lars Rudebeck, op_cit. page 8. 
propre dépendance. L'exode rural vers la ville commençait à se faire sentir et, dans la ville, les débouchés n'existaient pas." 1

En 1954, l'adoption de la loi du "Statut des indigènes", divisa les habitants de la colonie en deux groupes, les civilisés et les non-civilisés, comme en font foi les recensements effectués avant 1959 où la population était séparée en "civilizado" et "não-civilizado". L'Africain aspirant à la citoyenneté portugaise devait s'assimiler en répondant à ces conditions: 2

- être âgé de 18 ans et plus;

- savoir parler le portugais convenablement;

- avoir un revenu lui permettant de subvenir à ses besoins et à ceux de sa famille;

- avoir une bonne conduite et une certaine éducation; et,

- ne pas avoir déserté l'armée ou refusé de faire son service militaire.

De ce fait, l'immense majorité autochtone se voyant donc interdire le statut et les droits politiques et civiques portugais, se trouvait confinée à ses us et coutumes ancestrales. ${ }^{3}$

Le statut d'indigène soumettait l'Africain aux travaux forcés pour le public ou le privé, en plus de lui dénigrer le droit à toute propriété foncière autre que celle nécessaire à l'accomplissement de son mode de vie traditionnel.

Suite aux pressions internationales, dont la déclaration des Nations-Unies sur la décolonisation du 14 décembre 1960, le gouvernement portugais dut initier différentes réformes dans ses territoires africains. En 1961, l'abolition du "Statut des Indigènes" de

1 Carlos Lopes, "Ethnie, Etat et rapports de pouvoir en Guinée-Bissau", Itinéraires, notes et travaux, No. 22, mars 1982, Première Edition, Genève, page 6.

2 Ibid., page 22.

3 Ibidem. 
1954, accordait aux habitants de la Guinée, du Mozambique et de l'Angola, le statut de citoyen portugais au même titre que les habitants de la métropole. Ils devenaient ainsi sujets à la loi publique portugaise, en même temps qu'on leur reconnaissait la possibilité de conserver et de pratiquer leur droit coutumier. Malgré l'abolition, le 27 avril 1962, du travail forcé, le droit de vote est resté limité à ceux qui savaient lire et écrire le portugais, et qui pouvaient payer une taxe minimale de 200 escudos par anné. ${ }^{1}$

Parallèlement à cela, c'est au Portugal qu'une poignée d'intellectuels nationalistes guinéens fondèrent le 19 septembre 1956, le Partido Africano para a Indepedència da Guinée et das Ihas do Cabo Verde (P.A.I.G.C.), sous la bannière "unité et lutte". ${ }^{2}$ A ses débuts, le P.A.I.G.C. s'adressait d'abord aux populations urbaines. A partir de 1959, il élargit ses assises aux masses paysannes "dont l'expérience a montré qu'elles sont "la force physique principale de la lutte de libération", parce qu'elles constituent la majeure partie de la population".3

Le 3 août 1959, le massacre sanglant d'une cinquantaine de débardeurs en grève au port de Pidjiguiti à Bissau, marque le début de la mobilisation du P.A.I.G.C. vers la lutte armée. C'est avec l'attaque de la caserne de Tite en janvier 1963 que commença officiellement la révolte armée. Appliquant les principes de la guérilla, le P.A.I.G.C., sous le commandement d'Amilcar Cabral, sut mobiliser la population et, peu à peu, libérer d'importantes zones. La nomination à la tête de la Province à partir de 1968, du général Spinola qui favorisa de nombreuses réformes dans le but de renverser la vapeur de la machine révolutionnaire, tout

1 Ibid., pages 22-24.

2 Paulette Pierson-Mathy, La naissance de l'Etat par la guerre de libération nationale: le cas de la GuinéeBissau, Paris, U.N.E.S.C.O., 1980, page 17.

3 Ibid., page 19. 
comme l'assassinat d'Amilcar Cabral le 20 janvier 1973 a Conakry, ne purent empêcher la Guinée-Bissau, conjointement aux lles du Cap-Vert, d'acquérir son indépendance en 1974. ${ }^{1}$

Sous la présidence de Luis Cabral, frère d'Amilcar, la Guinée-Bissau entreprit alors une période de reconstruction nationale. Carlos Lopes dresse judicieusement le bilan de l'ère Luis Cabral:

"La réalité et le discours sont deux choses bien différentes. Nous attendons encore que la stratégie de développement de la Guinée-Bissau suive les principes énoncés par le PAIGC. A en croire les plans de développement élaborés au CECEP 2, tout irait pour le mieux. La Guinée-Bissau serait engagée dans un processus de self-reliance, voire dans une voie autogestionnaire donnant pleine priorité à l'agriculture sans pour cela bouleverser les structures sociales existantes. Certes, un soutien actif a été accordé aux réfugiés revenus au pays et les anciennes régions libérées ont été exemptées d'impôt en contrepartie des sacrifices consentis pendant la phase de la lutte armée.

De fait, cette politique (tout en admettant les nuances) a été plus ou moins fidelement suivie jusqu'en 1977. A partir de cette date, le régime Luis Cabral donne une orientation différente au pays. Le Congrès du PAIGC permet à une couche définie comme "intellectuelle" d'accéder aux postes d'organisation du PAIGC et d'y défendre ses intérêts petit-bourgeois." 3

La Guinée-Bissau s'engagera à cette époque dans un développement de type industriel, marqué de grands projets aucunement en accord avec ses potentialités du moment, et qui ne put qu'aboutir à des échecs. 4

1 J. R. de Benoit, "La Guinée-Bissau: le démarrage du nouvel Etat", Afrique Contemporaine, No. 83, janvierfévrier 1976, pages 12-13.

2 Le C.E.C.E.P. (Commissario do Estado a Coordenação Economica e ao Plano) est devenu ultérieurement le MICEPCI (Ministerio da Coordenação Economica do Plano e da Cooperação Internacional) et ensuite MICEP; il s'agit du Ministère du Plan.

3 Carlos Lopes, op. cit, page 61

4 Notons que la très grande majorité des investissements réalisés à ce moment résultèrent en des échecs. Ainsi, par exemple, à Bolama, une usine de tissus a été construite; elle n'a jamais fonctionné. Tant qu'à la fabrique de compote et de jus de fruits Titina Sila, elle ne marche jamais plus de deux mois par année (Carlos Lopes,"Ethnie, Etat et rapports de pouvoir en Guinée-Bissau", Itinéraires. notes et travaux, No. 22, mars 1982, Première Edition, Genève, page 62). Ailleurs dans le pays, le centre agro-alimentaire de Cumeré dont le budget de fonctionnement était de trois à quatre fois supérieur à celui de l'Etat (Yves, Brunet, Nancy Thede, Rapport de mission d'étude sur le projet d'étude pour la mise en place de structures régionales de développement (Guinée-Bissau), op, cit. page 13), et l'usine de coton de Bafata n'ont pas eu plus de succès. Ce virage technologique, axé vers l'exportation et dépendant de limportation de techniques étrangères et de ... suite 
Tout ceci, combiné à, paraît-il, un certain esprit dictatorial du président Luis Cabral et du "clan des cape-verdiens", a mené, dans la nuit du 14 novembre 1980, à un coup d'Etat (qualifié postérieurement par ses auteurs de "réajustement politique") dirigé par un héros de la lutte, le général Bernardo Vieira dit Nino, homme au charisme certain auprès de la population guinéenne. La conséquence la plus palpable de ce coup concerne sûrement la séparation de la Guinée-Bissau du Cap-Vert, et l'amorce d'une certaine campagne anti cape-verdienne depuis lors calmée. ${ }^{1}$

L'ère de Nino Vieira sera pour sa part marquée des pressions extérieures pour la rationalisation des dépenses de l'Etat, d'une plus grande ouverture sur l'Occident suivie d'une libéralisation du commerce, et de l'emphase toujours portée sur la nécessité de voir l'agriculture combler le déficit vivrier. Enfin, notons l'attention accordée aux efforts de développement des productions de base au détriment des projets industriels de grande envergure qui ne s'avérèrent que des échecs dans un pays constamment frappé de pénuries de toutes sortes. Officiellement du moins, le 14 novembre 1980 marque donc le passage d'une administration "technocratique" centralisant excessivement le pouvoir et l'arbitraire politique et policier aux mains des cape-verdiens, à un régime à tendance rurale populiste bissauguinéen, mené par une aile militaire devant aboutir à un régime civil pour 1982.2 Jusqu'à aujourd'hui, cependant, aucun fait ne nous permet de renier la persistance de allégeance militaire du régime de Bissau.

pièces de rechange, déborda rapidement les capacités de gestion et d'organisation locales (Ministère de la Coordination Economique, du Plan et de la Coopération Internationale, La production populaire en GuinéeBissau, Bissau, en collaboration avec OXFAM-Belgique, septembre 1985, Page 8).

1 Pour plus d'informations sur le sujet, on peut se référer aux journaux de l'époque dont le numéro 228 de la revue Afrique-Asie du lundi 8 décembre 1980 et du texte de Pierre François, La Guinée-Bissau après le coup d'Etat de novembre 1980, s.l., mai 1981, 28 pages.

2 Lars Rudebeck, "Problème de pouvoir populaire et de développement", Research Report, No. 63, 1982, chapitre IV, Le coup dEtat militaire du 14 novembre 1980. 


\subsubsection{Le pays aujourd'hui}

La Guinée-Bissau fait partie des pays les moins développés du monde avec une population jeune, un taux de mortalité supérieur à la moyenne mondiale et une espérance de vie des plus faibles.

\section{TABLEAU 1.1}

Principales données démographiques

Guinée-Bissau

\begin{tabular}{|lr|r|}
\hline Population (1986) & 891000 \\
\hline Densité (1986) par kilomètre carré & 24,7 habitants \\
\hline Distribution sexuelle (1985) & \\
& Hommes & $48,42 \%$ \\
& Femmes & $51,58 \%$ \\
\hline Projections de population pour & & \\
& 1990 & 987000 \\
& 2000 & 1229000 \\
\hline
\end{tabular}

Source: Encyclopaedia Britannica, 1987, Britannica Book of the Year. Chicago, Encyclopaedia Britannica Inc, 1987, page 660. 
TABLEAU 1.2

Distribution de la population totale du pays

selon l'âge

Guinée-Bissau

1986

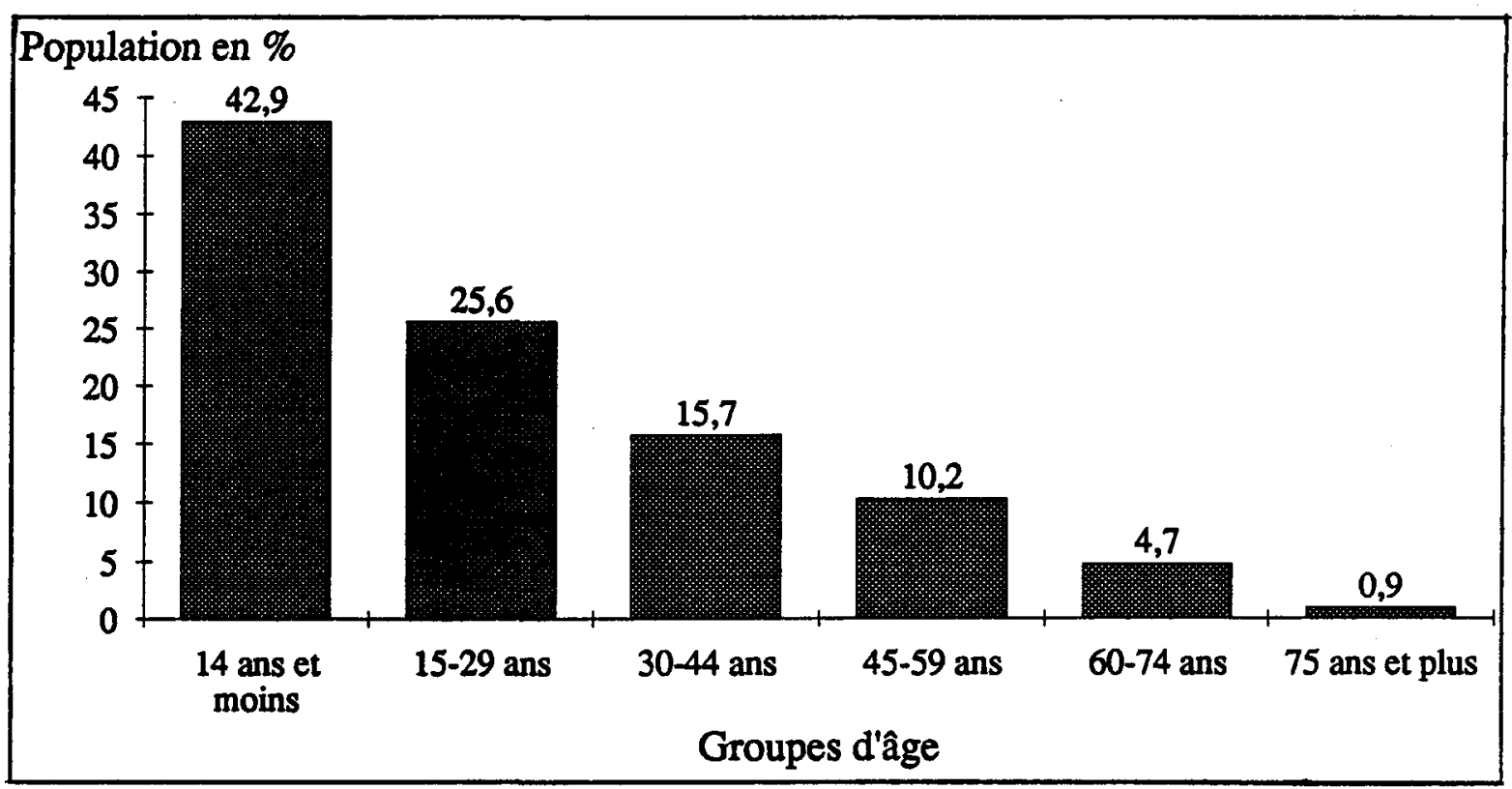

Source: Encyclopaedia Britannica, 1987. Britannica Book of the Year. Chicago, Encyclopaedia Britannica Inc, 1987, page 660. 
TABLEAU 1.3

Quelques données démographiques comparatives

Guinée-Bissau/monde

\begin{tabular}{|l|c|c|}
\cline { 2 - 3 } \multicolumn{1}{c|}{} & Guinée-Bissau & Monde \\
\hline $\begin{array}{l}\text { Taux de naissance } \\
\text { (sur 1000) } \\
\text { (1980 à 1985) }\end{array}$ & 41 & 27 \\
\hline $\begin{array}{l}\text { Taux de mortalité } \\
\text { (sur 1000) } \\
\text { (1980 à 1985) }\end{array}$ & 22 & 11 \\
\hline $\begin{array}{l}\text { Espérance de vie } \\
(1975 \text { à 1980) }\end{array}$ & 39,4 ans & - \\
\hline Femmes & 42,6 ans & - \\
\hline
\end{tabular}

Source: Encyclopaedia Britannica, 1987, Britannica Book of the Year. Chicago, Encyclopaedia Britannica Inc, 1987, page 660.

La population bissau-guinéenne a encore à faire face au lourd héritage de son passé colonial et de dix ans de lutte armée. Le réseau de transport (maritime et routier), déjà peu développé par l'administration coloniale, semble se détériorer constamment ${ }^{1}$ et, au niveau

1 Le réseau routier national est en effet dans un bien piteux état et n'assure qu'une liaison difficile et saisonnière entre le nord et le sud du pays. De plus, faut-il compter de longs détours pour relier le nord et le sud. Tant qu'aux liaisons routières permettant de sortir du pays, elles sont encore plus médiocres. Les routes pavées pour leur part dépassent à peine les $400 \mathrm{~km}$ (Carlos Lopes, A transição histórica na Guiné-Bissau: do ... suite 
maritime, les voies fluviales et côtières sont peu ou pas draguées et la signalisation fort déficiente. Quant aux installations de santé et d'éducation, leur âge avancé explique souvent leur état. En un sens, bien que l'aspect général du pays ne diffère guère de celui de l'ensemble des autres pays de cette région de l'Afrique, la Guinée-Bissau se caractérisait, jusqu'à tout récemment par un repli sur soi, fruit d'une orientation politique nationale de nonalignement. De plus, de par un manque de devises étrangères chronique, la Guinée-Bissau est aux prises avec une situation de pénuries régulières (pétrole, nourriture, biens de consommation et de production en général, etc.).

Lors de son premier plan quadriennal (1983-1986), le pays a commencé à libéraliser son économie et l'effet le plus perceptible en fut, à partir des années 1986 et 1987, une amélioration, tant qualitative que quantitative, de l'approvisionnement de la population. Cependant, parallèlement à cela, on a noté une dévaluation de la monnaie locale. Le peso guinéen (P.G.), créé en 1976, ${ }^{1}$ passait de 34 P.G. pour un dollar américain en 1980, à 44 en décembre 1983, 127,33 en décembre 1984, 150 en juin 1985, 650 en mai 1987 et à plus de 973 pesos pour un dollar américain en février $1988 . .^{2}$ Cette dévaluation monétaire aura évidemment été suivie d'une accentuation de l'inflation pour les biens de consommation courants, ce qui aura eu pour effet de toucher très durement les populations urbaines.

movimento de libertação nacional ao Estado, Mémoire présenté pour le Diplôme de Recherche en Etudes du Développement, Institut Universitaire d'Etudes du Développement, Genève, novembre 1982, page 120).

1 Lars Rudebeck, Guinea-Bissau: difficulties and possibilities of socialist orientation, Institute of Political Science ans the Working Group for the Study of Development Strategies (A.K.U.T.), Uppsala University, january 1978, page 17.

2 Groupement Lavalin/Delcanda, Enude générale sur les transports, s.l., Banque Africaine de Développement, juin 1980, volume I, Base socio-economique, page 9.2;

Philippe de Braconier, Elizabeth Drory, Rapport de mission de juin 1985: préćtude de l'tle de Paix de Bolama Huy, Iles de Paix, 1985, page 4;

Banco Nacional da Guiné-Bissau, Câmbios, 4/05/87, Bissau; et,

Banco Nacional da Guiné-Bissau, Câmbios. 1/02/88, Bissau. 


\subsubsection{Modes de production}

La Guinée-Bissau, nous le verrons, est un pays essentiellement agricole, plus de $80 \%$ de sa population vivant de l'agriculture, ${ }^{1}$ une agriculture d'autoconsommation. En campagne, on vit encore en accord avec ses traditions, en petites communautés n'engageant somme toute que bien peu de liens d'échanges entre elles. A l'instar de Carlos Lopes, nous pouvons remarquer l'existence de différents modes de production ${ }^{2}$ au sein de la formation sociale bissau-guinéenne, soit les modes de production communautaire, tributaire et capitaliste. ${ }^{3} \mathrm{La}$ présence de plusieurs modes de production trouve son explication dans la multiplicité ethnique et dans l'existence de mondes urbains et ruraux.

Samir Amin dit du mode de production communautaire, qu'il doit correspondre aux caractéristiques suivantes:

"1) l'organisation du travail, partie sur une base individuelle (celle de la "petite famille"), partie sur des bases collectives (celle de la "grande famille", du clan, du village), le moyen de travail essentiel, la terre, étant la propriété collective du clan, son usage libre à tous ses membres, mais selon des règles précises (utilisation de parcelles distribuées aux familles, etc.); 2) l'absence d'échanges marchands et, corrélativement, 3) la distribution du produit à l'intérieur de la collectivité selon des règles en relation étroite avec l'organisation de la parenté.

L'accès à la terre dans les communautés n'est pas nécessairement égalitaire. In l'est dans les plus primitives de ces communautés, mais, dans les autres, cet accès est hiérarchisé, certaines familles ou clans ayant droit à de meilleures parcelles, mieux situées par exemple, ou plus vastes." 4

1 S.E.P.C.I., Primeiro Plano Ouadrienal de Desenvolvimento Económico e Social 1983-1986: Relatório Geral, volume I, Republica de Guiné-Bissau, page 49.

2 Nous entendons par mode de production, une "combinaison particulière de rapports de production et de forces productives" (Samir Amin, Classe et nation dans l'histoire et la crise contemporaine, Paris, Les Editions de Minuit, 1979, page 22).

3 Carlos Lopes, Ethnie. Etat et rapports de pouvoir en Guinée-Bissau, op. cit., pages 24-32.

4 Samir Amin, Le développement inégal: essai sur les formes sociales du capitalisme périphérique, Paris, Les Editions de Minuit, 1973, page 10. 
Pour ce qui est du mode de production tributaire, il le définit ainsi:

"Le mode de production tributaire est caractérisé par la séparation de la société en deux classes essentielles: la paysannerie, organisée en communautés, et la classe dirigeante, qui monopolise les fonctions d'organisation politique de la société et perçoit un tribut (non marchand) sur les communautés rurales." 1

L'existence de rapports marchands est possible dans le mode de production tributaire; cependant, les échanges marchands ne sont pas dominants. ${ }^{2}$ Le mode de production communautaire pur ne devrait pas, selon Amin, présenter de rapports marchands. ${ }^{3}$ Dans la pratique, en Guinée-Bissau, où existe une économie monétaire au niveau national, ce principe ne pourra se vérifier. Ceci atteste la multiplicité des formes que ce mode de production peut revêtir, puisqu'il n'est, après tout, qu'un stade de transition assurant le passage du communisme primitif à une société de classes achevées. ${ }^{4}$ Le cas de la GuinéeBissau du moins, nous aura enseigné que les rapports marchands revêtent aux yeux de la population de ces modes de production une importance plus que marginale.

La différence entre ces deux modes de production réside, pour une bonne part, dans la séparation de la société tributaire en deux classes, la paysannerie, qui est propriétaire effective du sol, et l'aristocratie qui s'accapare les tâches d'organisation politique et de perception d'un tribut non-marchand provenant du "surplus formé de prestations de travail et d'impôts en nature réalisés à diverses étapes de la production et de la circulation".5 Comme on le sait, Samir Amin qualifie le mode de production communautaire de stade de transition

\footnotetext{
1 Ibid, page 10.

2 Samir Amin, Classe et nation dans l'histoire et la crise contemporaine, op. cit., page 21.

3 Samir Amin, Le développement inégal: essai sur les formes sociales du capitalisme périphérique, op. cit., page 10.

4 Ibid., page 9.

5 Gabriel Gagnon, Coopératives ou autogestion: Sénégal. Cuba. Tunisie, Montréal, Les Presses de l'Université de Montréal, 1976, page 27.
} 
entre une société à caractère "inachevé dans la formation des classes et de l'Etat", 1 basée sur les formes collectives de propriété, et une autre ayant des classes sociales, un Etat, des rapports de domination et d'exploitation, et une économie basée sur la valeur d'usage. Le consensus social nécessaire au fonctionnement et au maintien du mode de production tributaire reposera dans la religion, une religion d'Etat, différente de celle de terroir qui caractérise le mode de production communautaire, dans lequel l'idéologie de la parenté assure la présence et la reproduction des rapports de coopération et de domination. ${ }^{2}$

Il importe de se garder de répéter l'erreur courante assimilant mode de production communautaire à société égalitaire, et mode de production tributaire à société basée sur une certaine exploitation du travail d'autrui. Ainsi, comme l'explique Amin à propos du mode de production tributaire:

"... l'extraction du surproduit y est obtenue par des moyens non économiques, le producteur n'étant pas séparé de ses moyens de production. Un tel caractère oppose ce premier mode de classe au mode communautaire qui le précède. Là, le surproduit n'est pas approprié par une classe exploiteuse, il est centralisé par un groupe dirigeant pour être utilisé collectivement ou redistribué conformément aux exigences de la reproduction. La confusion entre d'une part les rapports de coopération et de domination et d'autre part les rapports d'exploitation, qui s'explique par le souci de combattre les simplifications naives assimilant le mode communautaire à un communisme primitif idyllique, est à l'origine de l'absence de la distinction entre le surproduit utilisé collectivement et le surproduit approprié par une classe exploiteuse. L'extraction du surproduit a donc la nature d'un tribut au profit de la classe exploiteuse; c'est pourquoi nous avons proposé d'appeler ce mode fondamental du second pallier le mode tributaire."

"La seconde caractéristique du mode tributaire est que l'organisation essentielle de la production y est fondée sur la valeur d'usage et non sur la valeur d'échange. Le produit conservé par le producteur est lui-même directement valeur d'usage destinée à la consommation et, pour l'essentiel, à l'autoconsommation. Mais le produit extrait par la classe exploiteuse est lui aussi directement valeur d'usage. C'est dire que l'essence de ce mode tributaire est de

1 Samir Amin, Classe et nation dans l'histoire et la crise contemporaine, op. cit., page 20.

2 Ibid., pages 17-59. 
fonder une économie naturelle, sans échanges, sinon sans transferts (le tribut en est un), et sans redistributions.

On constate certes l'existence d'échanges non monétaires, ou même monétaires, dans toutes les formation tributaires. Toutefois, ces échanges ne sont qu'accessoirement marchands, c'est-à-dire qu'ils ne sont pas fondés sur la valeur d'échange (la loi de la valeur) mais sur la valeur d'usage (les utilités comparatives)." 1

La reconnaissance de l'existence en Guinée-Bissau de ces modes de production précapitalistes revêt une importance capitale dans la compréhension de la réalité bissauguinéenne et, nous permettra de mieux saisir la culture locale en regard des objectifs du mémoire. Il importe cependant toujours de garder à l'esprit que la survivance jusqu'à ce jour, de l'économie de subsistance en Guinée-Bissau dans ces deux modes de production, peut tendre à prouver "la résistance socio-économique qui anime l'Afrique en général et la GuinéeBissau en particulier". 2 Bien que la distinction entre les ethnies appartenant à l'un ou l'autre mode de production soit parfois difficile à établir, notons que les ethnies de religion islamique telles les Foulas et les Mandingues, relèvent du mode de production tributaire, alors que la plupart des autres, animistes, à sociétés dites horizontales en général, appartiennent au mode de production communautaire. Amilcar Cabral en fait d'ailleurs état, qualifiant de "semiféodal" et de "sans Etat", ce que nous appelons respectivement mode de production tributaire et mode de production communautaire:

"Nous devons faire des distinctions entre les diverses situations, sans toutefois les opposer.

Ainsi, à la campagne, nous trouvons d'une part le groupe que nous considérons comme semi-féodal, représenté par les Foulas, et d'autre part celui des Balantes, que nous appellerons société "sans Etat". Il existe différentes situations intermédiaires entre ces deux groupes ethniques extrêmes. Il y a ainsi parmi les animistes - alors qu'on trouve une coïncidence entre semi-féodalisme et islamisme, et aucune organisation d'Etat parmi les animistes - un groupe

\footnotetext{
1 Ibid, pages 58-59.

2 Carlos Lopes, op.cit. page 25.
} 
ethnique, les Mandjaks, qui, à l'arrivée des Portugais, entretenaient déjà des rapports que l'on pourrait qualifier de féodaux." 1

Quoique l'objet du mémoire ne soit pas ethnologique, nous reviendrons sur ces considérations dans un chapitre ultérieur sur les sociétés verticales et horizontales.

Le mode de production capitaliste, quant à lui, n'est présent que dans les villes. Son importance est somme toute relative puisque, d'après Lopes, en 1977, 56\% du P.I.B. se situait dans le secteur de l'autosubsistance, des rapports non marchands. ${ }^{2}$

Dans le cas de la Guinée-Bissau, l'existence du capitalisme relève surtout du secteur tertiaire dont l'hypertrophie est normalement l'un des traits des pays du tiers-monde. ${ }^{3} \mathrm{~A}$ première vue, le tertiaire ne semble pas trop mobiliser la population active du pays. Cependant, il emploie la quasi-totalité du salariat national dans des activités somme toute peu productives.

Le Tableau 1.4 nous permet de constater l'aspect marginal du salariat, retranché à une mince couche de la population active concentrée dans le tertiaire. Le recensement de 1979 chiffrait la population active du pays à 198583 personnes, ${ }^{4}$ soit seulement $25,4 \%$ de la population totale. Ce faible taux s'explique par le fait qu'il exclut de la population active, les jeunes de moins de 15 ans et les personnes de plus de 60 ans, ce qui fait passer la population de 780985 personnes à 402 251, de même que la "femme au foyer", en opposition à la femme sur le marché du travail, qui représente la très grande majorité des femmes du pays, ce

1 Amilcar Cabral, "Brève analyse de la structure sociale de la Guinée "portugaise" pensée polique d'Amilcar Cabral, Montréal, Dossier S.U.C.O., Secteur Education, page 16.

2 Ibid., page 31.

3 Samir Amin, Le développement inégal: essai sur les formes sociales du capitalisme périphérique, op. cit., page 173.

4 Ministerio da Coordenação Económica e Plano, Recenseamento geral da populacão e da habitacão. 16 de abril de 1979. resultados provisórios (fase II), Bissau, Departamento Central de Recenseamento, 1979, page 171. 
qui porte le nombre à moins de 200000 . L'exclusion des femmes de la population active explique d'ailleurs les différences importantes que l'on note entre les différentes sources de références. Ainsi est-il intéressant de remarquer que Braconier et Drory évaluent la population active du pays à 400000 personnes. ${ }^{1} \mathrm{Ce}$ chiffre correspond beaucoup plus à ce que nous avons calculé et, de ce fait, nous semble plus conforme à la réalité, les femmes, tout comme les enfants de moins de 15 ans, participant activement au processus de production familial soit par leur implication dans la riziculture, au maraîchage, à la pêche et aux productions artisanales, ou encore par leur quasi-monopole de la cueillette, si l'on excepte la cueillette du régime de palme qui s'effectue uniquement par l'homme. C'est d'ailleurs pourquoi l'étude de S.U.C.O.-C.R.A.D. définissait la population active comme l'ensemble des adultes valides des deux sexes ${ }^{2}$ et non, par exemple, suivant notre conception canadienne, l'ensemble des "personnes de 15 ans et plus qui occupent un emploi ou effectuent des démarches pour en occuper un". ${ }^{3}$ Considérée sous cet angle, la définition de la population active que véhicule le recensement de 1979 correspond à la vision occidentale assimilant population active à marché du travail. Une telle conception de la population active n'est, de notre avis, aucunement en accord avec la réalité de la Guinée-Bissau, la population y vivant avant tout en campagne selon un mode de vie plus ou moins autarcique. Ainsi ne pouvons-nous qu'émettre de sérieuses réserves à l'endroit de ces résultats du recensement et allons donc adopter comme

1 Philippe de Braconier, Elizabeth Drory, Rapport de mission de juin 1985: préćtude de l'ile de Paix de Bolama op. cit. Annexe I, page 3.

2 S.U.C.O.-C.R.A.D. Le développement rural intégré de la région de Bolama République de Guinée-Bissau, Université Laval, Maîtrise en Aménagement du Territoire et Développement Régional, 1982, Volume II, page 36.

3 Ministère de la Main-d'Oeuvre et de la Sécurité du Revenu, Concepts et éléments d'analyse du marché du travail, s.l., Service des Etudes Régionales et de l'Analyse Conjoncturelle, Direction de la Recherche, janvier 1985 , page 38. 
vraisemblable les estimations du Ministère du Plan qui portaient la population active à environ 450000 personnes, soit $56 \%$ de la population totale du pays. 1

Le Tableau 1.4 est basé sur une estimation effectuée à partir de la distribution en pourcentage de la population active et de la population salariée par secteurs d'activité, telle que présentée par le Ministère du Plan. Nous avons déduit, grâce à différentes sources, le nombre total de salariés et de personnes appartenant à la population active. L'importance quantitative de la population active que nous présentons sur ce tableau provient des estimations du Ministère du Plan que nous avons précédemment exposées. La population salariée a, pour sa part, été calculée d'après les chiffres du Groupement Lavallin/Delcanda selon lesquels la fonction publique occupe 15051 personnes, soit $61,7 \%$ de l'ensemble des salariés. De là pouvons-nous établir que la population salariée du pays se chiffre à 24394 personnes, ${ }^{2}$ ce qui se rapproche des estimations de Braconier et Drory qui évaluaient cette population à $25000 .^{3}$

Ces calculs nous permettent de dresser un portrait quantitatif approximatif de la population active et salariée en Guinée-Bissau au début des années 1980 et de constater que la fonction publique emploie presque $75 \%(74,3 \%)$ des salariés du tertiaire. Ces derniers représentent, pour leur part, 49,99\% de l'ensemble des travailleurs classés dans le tertiaire. On comprendra que la différence entre les membres salariés du tertiaire et ceux de l'ensemble du secteur, repose dans l'importance qu'ont le commerce privé et toute la gamme de petits propriétaires de services (cordonniers, coiffeurs, couturiers, etc.). De même, $85,55 \%$ des travailleurs du secondaire ne sont pas salariés; il s'agit d'artisans à leur compte. Quant à la

1 MICEPCI. Primeiro Plano Ouadrienal de Desenvolvimento Económico e Social 1983-1986. Bissau, Grupo de Trabalho Sectorial (Agricultura, Pecúaria e Florestas), abril de 1982. A: Relatório da situação actual, page 2.

2 Groupement Lavalin/Delcanda, Etude générale sur les transports, op. cit., page 2-35.

3 Philippe de Braconier, Elizabeth Drory, op.cit., Annexe I, page 3. 
TABLEAU 1.4

Distribution et estimation de la population active et salariee selon le secteur d'activité Guinée-Bissau $1979-1980$

\begin{tabular}{|c|c|c|c|c|}
\hline \multirow{2}{*}{ Secteur d'activité } & \multicolumn{2}{|c|}{ Population active } & \multicolumn{2}{|c|}{ Population salariée } \\
\hline & $\%$ & Nombre & $\%$ & Nombre \\
\hline Primaire & 88 & 396000 & 7 & 1708 \\
\hline Secondaire & 3 & 13500 & 8 & 1951 \\
\hline Tertiaire & 9 & 40500 & 83 & 20247 \\
\hline Activités mal définies & - & - & 2 & 488 \\
\hline Total & 100 & 450000 & 100 & 24394 \\
\hline
\end{tabular}

Source: Secretaria de Estado da Presidência para os Assuntos Economicos e Cooperação Internacional, Guine-Bissau; sintese da situação económico-financeira e perspectivas de médio prazo, Bissau, fevereiro de 1987, page 4; et, compilation interne pour les chiffres absolus. 
très grande majorité des membres du secteur primaire qui ne sont pas salariés $(99,6 \%)$, il s'agit pour l'ensemble, de paysans et de pêcheurs. Le Tableau 1.4 nous permet donc de constater l'aspect très marginal du salariat au sein de la population active bissau-guinéenne. Le salariat ne toucherait ainsi dans le pays qu'une mince tranche de 3,1\% de la population totale.

Les quelques salariés du secteur primaire relèvent des fermes d'Etat ("Grangas") et des quelques 300 exploitants propriétaires terriens ("Ponteiros") qui possèdent de 15000 à 20000 hectares de terre dans le pays. ${ }^{1}$ Notons cependant que ces "ponteiros" n'ont pas tous recours à l'engagement de main-d'oeuvre pour exploiter leurs terres, mais plutôt, comme dans les cas que nous avons pu observer à Bolama, demander une rente aux paysans qui désirent cultiver leur terre. Dans les régions côtières et insulaires, plusieurs hommes, apprentis et pêcheurs, ne possédant pas d'embarcation, offrent leurs services à des propriétaires de pirogues ou de navires et deviennent alors salariés.

Au niveau du secteur secondaire, quelques usines de transformation fonctionnent et embauchent du personnel (congélation de poisson et de crustacés, fabrication de jus et de compote, etc.).Quant à la population salariée du secteur tertiaire, elle se compose pour 74,34\% d'employés de l'Etat, ${ }^{2}$ le reste se divisant entre les employés des commerces privés et les personnes engagées par des privés pour diverses fonctions, soit par exemple comme employés ménagers ou employés de projets.

1 SCET-AGRI, Etude des prix et incitations aux producteurs ruraux, MICEPCI, République de Guinée-Bissau, janvier 1986, page 25.

2 Calculé à partir du chiffre de 15051 fonctionnaires (Groupement Lavalin/Delcanda, op. cit, page 2-35) et du total des travailleurs du secteur tertiaire (40 500). 
Il est évident, à la lumière de ces quelques chiffres, que le salariat et, par extension, le capitalisme et son mode de vie axé sur la production et la consommation, semblent peu importants en Guinée-Bissau. Tout au long du présent ouvrage, il faudra garder à l'esprit le faible ancrage actuel du mode de production capitaliste au sein de la formation sociale 1 bissau-guinéenne.

\subsubsection{Importations et exportations}

Au niveau économique, la situation nationale n'est guère positive et le pays est toujours à la recherche de l'autosuffisance alimentaire. Sa balance commerciale est nettement déficitaire (voit Tableau 1.5), son déficit global représentait 29,9\% du P.I.B. global en 1985 alors que le déficit courant comptait pour $10,7 \%$ du P.I.B. à la même période. ${ }^{2}$ Face à cette situation, il n'est pas étonnant d'apprendre que le pays est continuellement en quête de devises étrangères pour satisfaire ses besoins d'importation qui ont d'ailleurs connu une augmentation marquée depuis la fin de la lutte.

1 Nous adoptons la définition de Samir Amin des formations sociales:

"Les formations sociales sont donc des structures concrètes [en occurrence la Guinée-Bissau], organisées, caractérisées par un mode de production dominant [le capitalisme] et l'articulation autour de lui d'un ensemble complexe de modes de production [communautaire et tributaire] qui lui sont soumis." (Samir Amin, Le développement inégal: essai sur les formes sociales du capitalisme périphérique, op cit., page 12).

2 Secretaria de Estado da Presidência para os Assuntos Economicos e Cooperação Internacional, Guine-Bissaul: sintese da situacão económico-financeira e perspectivas de médio prazo, Bissau, fevereiro de 1987, page 13. 


\section{TABLEAU 1.5}

Balance commerciale 1981-1985

Guinée-Bissau

(en millions de dollars américains)

\begin{tabular}{|l|r|r|r|r|r|}
\cline { 2 - 5 } \multicolumn{1}{c|}{} & \multicolumn{1}{c|}{1981} & \multicolumn{1}{c|}{1982} & \multicolumn{1}{c|}{1983} & \multicolumn{1}{c|}{1984} & \multicolumn{1}{c|}{1985} \\
\hline Exportations (F.O.B.) & 13,9 & 11,8 & 8,6 & 17,4 & 11,6 \\
Importations (F.O.B.) & -52 & $-69,4$ & $-58,4$ & $-60,1$ & $-59,5$ \\
Balance commerciale & $-38,1$ & $-57,6$ & $-49,8$ & $-42,7$ & $-47,9$ \\
\hline
\end{tabular}

Source: Secretaria de Estado da Presidência para os Assuntos Economicos e Cooperação Internacional, Guine-Bissau: sintese da situacão económico-financeira e perspectivas de médio prazo, Bissau, fevereiro de 1987, page 4.

L'étude de l'évolution des importations et des exportations de la période 1960 à 1981 (Tableaux 1.5 et 1.6, Graphique I) montre la tendance quasi-exponentielle de l'accroissement des importations durant la décennie de 1970. Le Tableau 1.5 sur la balance commerciale du pays permet par contre de constater une certaine stabilisation tant des importations que des exportations de 1981 à 1985, ce qui s'explique vraisemblablement par le changement de régime consécutif au "réajustement politique" de 1980 et à l'adoption du Premier Plan Quadriennal (1983-1986). 
TABLEAU 1.6

Balance commerciale

Importations et exportations annuelles

Guinée-Bissau

1960-1981

(en millions de contos ${ }^{1}$ )

\begin{tabular}{|c|c|c|c|}
\hline Annee & Importations & Exportations & $\begin{array}{c}\text { Balance } \\
\text { commerciale }\end{array}$ \\
\hline 1960 & 321,77 & 125,89 & $-195,88$ \\
1961 & 292,11 & 248,13 & $-43,98$ \\
1962 & 342,55 & 221,91 & $-120,64$ \\
1963 & 407,16 & 195,59 & $-211,57$ \\
1964 & 431,67 & 182,95 & $-248,72$ \\
1965 & 419,32 & 123,67 & $-295,65$ \\
1966 & 433,51 & 99,27 & $-334,24$ \\
1967 & 471,85 & 107,6 & $-364,25$ \\
1968 & 506,66 & 103,22 & $-403,44$ \\
1969 & 672,26 & 124,21 & $-548,05$ \\
1970 & 786,04 & 106,42 & $-679,62$ \\
1971 & 879,17 & 67,68 & $-811,49$ \\
1972 & 866,84 & 82,01 & $-784,83$ \\
1973 & 1076,69 & 93,95 & $-982,74$ \\
1974 & 1095,35 & 88,38 & $-1006,97$ \\
1975 & 964,53 & 180,63 & $-783,9$ \\
1976 & 1106,32 & 186,88 & $-919,44$ \\
1977 & 1173,06 & 404,19 & $-768,87$ \\
1978 & 1726,41 & 422,64 & $-1303,77$ \\
1979 & 2067,65 & 479,63 & $-1588,02$ \\
1980 & 1859,86 & 382,32 & $-1477,54$ \\
1981 & 1859,95 & 524,9 & $-1335,05$ \\
\hline Moyennes & 898,21 & 206,91 & $-691,3$ \\
\hline
\end{tabular}

Source:Nations-Unies, Annuaire statistique du commerce international, 1981, Volume I, Commerce par pays, New York, Publication des Nations-Unies, 1983, page 434.

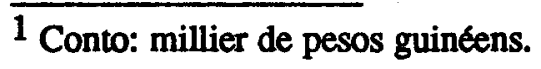




\title{
GRAPHIQUE I
}

\author{
Importations et exportations annuelles \\ Guinée-Bissau \\ 1960-1981 \\ (en millions de contos par année)
}

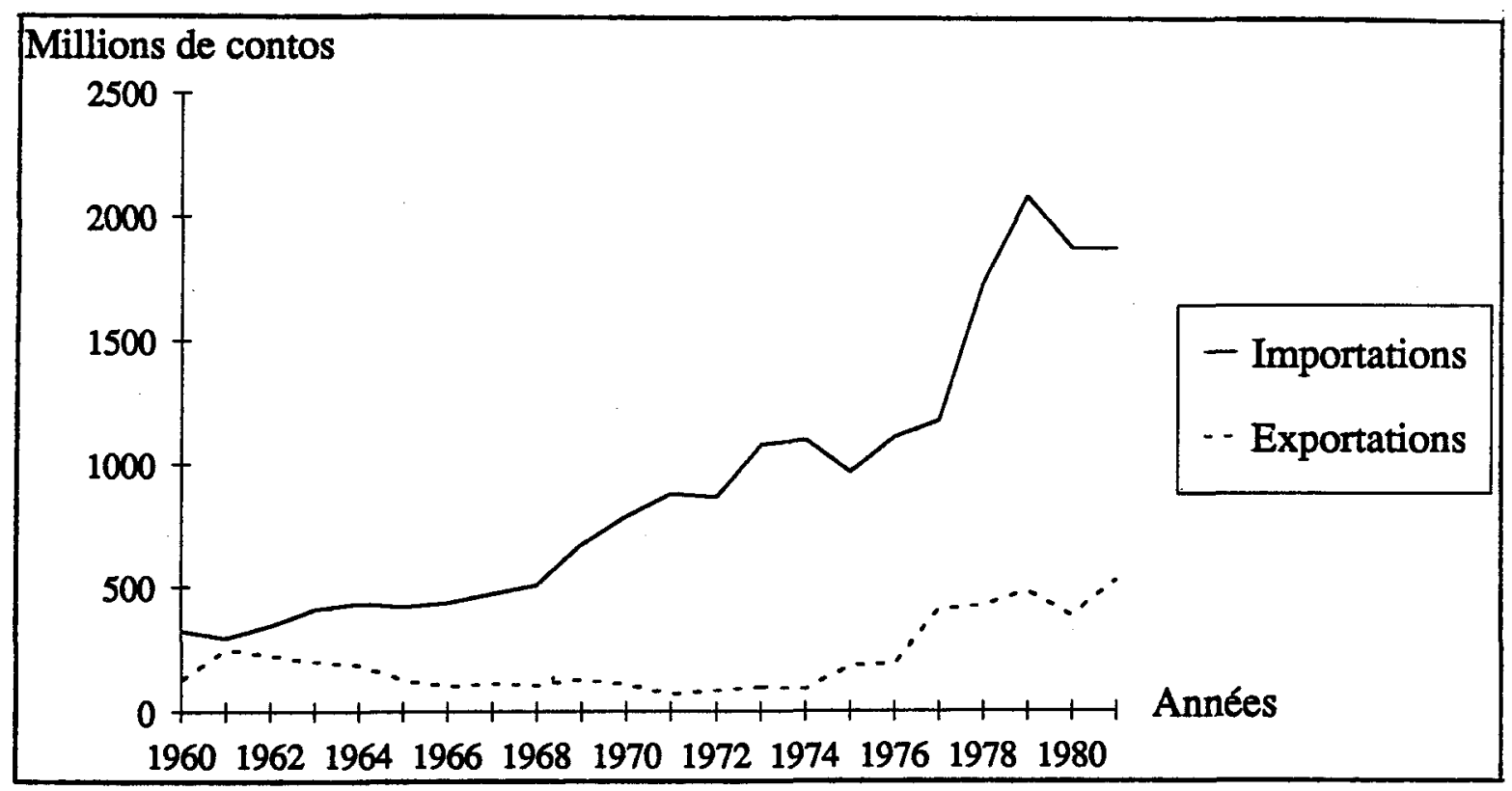

Effectué à partir de: Nations-Unies, Annuaire statistique du commerce international, 1981, Volume I, Commerce par pays, New York, Publication des Nations-Unies, 1983, page 434 . 
La structure des exportations du pays n'a guère changé depuis l'époque coloniale. Carlos Lopes souligne qu'en 1964, les arachides et les noix de coco comptaient pour 92,7\% des exportations de la Guinée. ${ }^{1}$ Selon le Tableau 1.7, la Guinée-Bissau des années 1980 n'est toujours qu'un réservoir de ressources naturelles où la transformation sur place est inexistante dans la plupart des cas.

\section{TABLEAU 1.7}

Structure des exportations, Guinée-Bissau

1983-1985 (en \% du total)

\begin{tabular}{|l|r|r|r|}
\cline { 2 - 4 } \multicolumn{1}{c|}{} & \multicolumn{1}{c|}{1983} & \multicolumn{1}{c|}{1984} & \multicolumn{1}{c|}{1985} \\
\hline Produits agricoles & 72,2 & 78,6 & 69,5 \\
Produits forestiers & 4,6 & 2,3 & 3,2 \\
Produits de la mer & 23,2 & 19,1 & 27,3 \\
\hline \multicolumn{1}{|c|}{ Total } & 100 & 100 & 100 \\
\hline
\end{tabular}

Source: Secretaria de Estado da Presidência para os Assuntos Economicos e Cooperação Internacional, Guine-Bissau; sintese da situação económico-financeira e perspectivas de médio prazo, Bissau, fevereiro de 1987, page 17.

1 Carlos Lopes, A transição histórica na Guiné-Bissau: de movimente de libertação nacional ao Estado, op. cit., pages 120-121. 
Une étude plus poussée indique qu'en 1977 par exemple, le pays avait exporté les produits suivants:

\section{TABLEAU 1.8}

Nature des exportations

Guinée-Bissau en 1977

(en \% de la valeur totale)

\begin{tabular}{|l|r|}
\hline \multicolumn{1}{|c|}{ Produit } & \multicolumn{1}{c|}{ Pourcentage } \\
\hline Arachides & 60,3 \\
\hline $\begin{array}{l}\text { Crustacés et } \\
\text { mollusques }\end{array}$ & 13,1 \\
\hline Coprah & 12,5 \\
\hline Poissons & 6,1 \\
\hline Noix de cajou & 2,4 \\
\hline $\begin{array}{l}\text { Autres (fruits, légumes, } \\
\text { bois, huile de palme, } \\
\text { cire d'abeille) }\end{array}$ & 5,6 \\
\hline Total & 100 \\
\hline
\end{tabular}

Source:Nations-Unies, Annuaire statistique du commerce international, 1981, Volume I, Commerce par pays, New York, Publication des Nations-Unies, 1983, page 438, compilation interne. 
Une source plus récente confirme le rôle de "pays-ressource" conféré à la GuinéeBissau: en 1983, le pays exporta pour un portion de $66,1 \%$ des valeurs totales de produits agricoles (arachides, noix de cajou, fruits et légumes), de poissons, mollusques et crustacés (crevettes entre autres) pour $23,7 \%$ et enfin pour $4,5 \%$ de bois. ${ }^{1}$

Une infime partie des exportations uniquement est touchée par une quelconque transformation. Ainsi, en 1977, comparativement à la valeur totale des exportations, seuls les produits suivants étaient d'une manière ou d'une autre transformés et ce, dans les proportions suivantes: poissons, crustacés et mollusques, 19,2\% (congélation, éviscération, empaquetage), bois équarri ou dégrossi, $1,5 \%$, tourteaux, $1,7 \%$, huiles et graisses animales et végétales, $0,9 \%$ et, enfin, divers articles manufacturés en bois (artisanat et autres) comptant pour $0,7 \%$ de la valeur totale des exportations. ${ }^{2}$ Sept ans plus tard, pour 1984 et 1985, le scénario avait peu évolué, la Guinée-Bissau exportant, par rapport à la valeur totale, pour plus de $95 \%$ en 1984 et $99 \%$ en 1985 , des produits des trois groupes suivants: produits agricoles $(74,5 \%$ en 1984 et $68,6 \%$ en 1985$)$, produits de la mer (18,6\% en 1984 et $28 \%$ en $1985)$ et produits forestiers $(2,32 \%$ en 1984 et $3,03 \%$ en 1985$){ }^{3}$

La faible diversité des exportations du pays, de même que leur nature (relevant presqu'exclusivement du secteur primaire), le place dans une situation de vulnérabilité extrême, celui-ci devenant dépendant au niveau de ses exportations des aléas climatiques par exemple. Peuvent également intervenir en sa défaveur, d'autres variables telles la fluctuation des prix mondiaux de ces produits et la valeur du peso guinéen. Ainsi, comme le note le

1 Encyclopaedia Britannica, 1987. Britannica Book of the Year, Chicago, Encyclopaedia Britannica Inc., 1987, page 660.

2 Compilation effectuée à partir de Nations-Unies, "Exportations spéciales FOB par produits", Annuaire statistique du commerce international. 1981, New York, Publication des Nations-Unies, 1983, Volume I, Commence par pays, page 438.

3 Ministério do Desenvolvimento Rural e Pescas, Anuário estatístico 1985, Gabinete de Planeamento, Republica da Guine-Bissau, abril de 1985, page 42. 
Ministère du Plan, jusqu'à la fin de 1983, une sur-évaluation du peso pour des fins d'appui aux producteurs nationaux, aura été préjudiciable aux exportations. A la fin de 1983, une dévaluation de $100 \%$ du P.G. aura eu pour effet de stimuler les exportations. ${ }^{1}$ Il convient cependant de relativiser l'impact des politiques monétaires en Guinée-Bissau puisque, outre le non-recouvrement des coûts de production des produits agricoles par les prix pratiqués officiellement, le manque de confiance des producteurs dans la capacité du marché national de leur permettre une consommation intéressante, n'encouragent guère les cultures et les productions d'exportation. ${ }^{2}$ De plus, cette analyse du Ministère du Plan sur la sur-évaluation de la monnaie découlant d'une politique volontaire d'augmentation des prix pratiqués aux producteurs, omet qu'entre 1977 et 1981, la masse monétaire du pays s'accrût en moyenne de plus de 30\% par an et cet accroissement, conjugué à un crédit de la Banque Centrale à l'Etat suivant un taux annuel de 45\%, entraîna un taux d'inflation passant de $9 \%$ en 1977 a $30 \%$ en 1980-1982. ${ }^{3}$ Le taux d'inflation a été encore plus marqué lors des dévaluations monétaires successives de 1986 à 1988.

1 Secretaria de Estado da Presidência para os Assuntos Economicos e Cooperação Internacional, op. cit.page 17.

2 SCET-AGRI, op cit. page 73.

3 Americo, Ramos dos Santos, "Estabilização e subdesenvolvimento: liçð̄es da Guiné-Bissau", Economia e Socialismo, No. 60, janeiro-março de 1984, page 52. 
TABLEAU 1.9

Structures des importations

1983-1985

Guinée-Bissau

(en \% de la valeur totale)

\begin{tabular}{|l|r|r|r|}
\hline \multicolumn{1}{|c|}{ Produit } & \multicolumn{1}{c|}{1983} & \multicolumn{1}{c|}{1984} & \multicolumn{1}{c|}{1985} \\
\hline Produits alimentaires & 21,2 & $*$ & 18,8 \\
Boissons et tabac & 1,9 & $*$ & 2 \\
Autres biens de consommation & 23,2 & $*$ & 18,4 \\
Produits pétroliers & 11,9 & 14,1 & 12,6 \\
Matériel de construction & 11 & 13,3 & 19,6 \\
Matériel de transport & 11,6 & 10,2 & 7,6 \\
Machinerie et équipement électrique & 11,6 & 14,1 & 13,8 \\
Autres & 7,6 & 7,4 & 7,2 \\
\hline \multicolumn{1}{|c|}{ Total } & 100 & 100 & 100 \\
\hline
\end{tabular}

*: La somme des trois égale $40,9 \%$.

Source: Secretaria de Estado da Presidência para os Assuntos Economicos e Cooperação Internacional, Guine-Bissau: sintese da situação económico-financeira e perspectivas de médio prazo, Bissau, fevereiro de 1987, page 17. 
Le Tableau 1.9 nous renseigne de façon non-équivoque sur la nature des importations du pays visant à répondre aux besoins de consommation d'un pays qui produit peu, de façon non diversifiée et qui, malgré sa vocation agricole, n'est pas autosuffisant sur le plan alimentaire. Ainsi, le déficit céréalier du pays est important et force la Guinée-Bissau à recourir à l'assistance alimentaire de la communauté internationale.Le déficit alimentaire national en matière de riz, base de l'alimentation de la population, pour la période de 1982 à 1987 correspondait à un volume estimé à $24,6 \%$ de la production nationale annuelle avec une pointe de $84,2 \%$ de celle-ci en 1982 et une prévision de 30,8\% pour 1987. Pour les autres céréales (mil, sorgho, blé), le déficit atteignait une moyenne de $20,7 \%$ du total de la production, ce qui correspondait à un maximum de 40,1\% en 1983 et un minimum estimé de 7,75\% de la production nationale pour $1987 .{ }^{1}$ Le pays devra donc avoir recours aux importations celles-ci se divisant en deux groupes, selon qu'elles soient d'ordre commercial ou proviennent de l'aide internationale, cette dernière catégorie se subdivisant en deux, l'aide commercialisable et l'aide non-commercialisable ('aide alimentaire non-commercialisable est nettement moins importante que celle commercialisable). ${ }^{2}$

Le Tableau 1.10 fait état de la disponibilité totale du pays en matière de riz et nous permet de constater que, bien que la production nationale annuelle de riz ait eu tendance à augmenter dans la période 1977-1985, l'aide alimentaire a aussi crâ, et ce de façon beaucoup plus continue que la production agricole elle-même. Sur ce plan, on peut dire que la GuinéeBissau ne se démarque pas de l'aide alimentaire mondiale, bien au contraire...

1 Secretaria de Estado da Presidência para os Assuntos Economicos e Cooperação Internacional, op. cit. Annexe II.

2 En 1987, sur les 32718 tonnes de riz importées, 20000 étaient d'ordre commercial alors que des 12718 tonnes fournies par l'aide alimentaire, 2246 tonnes ne pouvaient être vendues, le reste (10 472 tonnes) pouvant l'être (Ministério do Desenvolvimento Rural e Pescas, op. cit., page 44). 
TABLEAU 1.10

Disponibilité totale de riz

Importations et production nationale

1977-1985

Guinée-Bissau

(en tonnes)

\begin{tabular}{|l|r|r|r|r|r|}
\hline \multicolumn{1}{|c|}{ Année } & $\begin{array}{c}\text { Importations } \\
\text { commerciales }\end{array}$ & $\begin{array}{c}\text { Aide } \\
\text { alimentaire }\end{array}$ & $\begin{array}{c}\text { Total des } \\
\text { importations }\end{array}$ & $\begin{array}{c}\text { Production } \\
\text { nationale }\end{array}$ & $\begin{array}{c}\text { Disponibilité } \\
\text { de riz }\end{array}$ \\
\hline 1977 & 4500 & 11559 & 16059 & 37050 & 53109 \\
1978 & -5010 & 15010 & 60125 & 75135 \\
1979 & 500 & 16421 & 16921 & 46150 & 63071 \\
1980 & 5050 & 8634 & 13684 & 21775 & 35459 \\
1981 & 5000 & 28000 & 33000 & 52000 & 85000 \\
1982 & 5000 & 17640 & 22640 & 72760 & 95400 \\
1983 & 678 & 24156 & 24834 & 51250 & 76084 \\
1984 & 6000 & 23326 & 29326 & 58000 & 87326 \\
1985 & 20000 & 12718 & 32718 & 63680 & 96578 \\
\hline Moyennes & & & & & 74129,1 \\
\hline annuelles & 5841 & 17496 & 22688 & 51421,1 & \\
\hline
\end{tabular}

Source: Ministério do Desenvolvimento Rural e Pescas, Anuário estatístico 1985, Gabinete de Planeamento, Republica da Guiné-Bissau, abril de 1986, page 45; et, compilation interne. 
Il est intéressant, à ce stade-ci, de noter que la population bissau-guinéenne a un régime alimentaire presqu'essentiellement axé sur la consommation du riz, à un point tel qu'à partir de 1987, il fut décidé, pour tenir compte des particularités mêmes de la population du pays, de porter les besoins annuels du Guinéen moyen de $99,64 \mathrm{~kg}$ de riz à $110 \mathrm{~kg}$. La production nationale de riz ne suffit pas comme nous l'avons déjà remarqué et, en 1987 par exemple, le pays a eu besoin de plus de 100000 tonnes pour nourrir sa population alors qu'il n'en produisait que 78000 tonnes, soit un manque à gagner de plus de 21000 tonnes. ${ }^{1}$ Cette situation contraste avec celle prévalant lors de la période coloniale où la Guinée exportait en moyenne 2000 tonnes de riz par année et connaissait, dit-on, l'autosuffisance en matière de culture de riz, avec une production qui, en 1953 par exemple, année d'une enquête agricole, se chiffrait à 100277 tonnes. 2

La destination de ses exportations de même que la provenance de ses importations démontrent que la Guinée-Bissau entretient encore de forts liens avec son ancienne métropole coloniale dont elle a conservé la langue, le portugais, pour en faire sa première langue officielle, devant le français (seconde langue officielle et aussi langue officielle de la plupart de ses voisins frontaliers) et sa langue véhiculaire, le créole guinéen aussi parlé en Casamance, région sud du Sénégal, et au Cap-Vert. Les exportations bissau-guinéennes se dirigeaient en 1987 par exemple, vers le Portugal (42,1\% de la valeur totale de celles-ci), la France $(17,2 \%)$, la Belgique et le Luxembourg $(8,7 \%)$ et la Hollande $(7,8 \%)$. Au niveau des

1 Ministère du Plan, Estimation du déficit céréalier en 1988 et requête d'assistance introduite auprès de la communauté intemationale, Bissau, décembre 1987, 5 pages: nous avons calculé l'estimation du déficit céréalier à partir des informations contenues dans ce document.

2 MICEPCI, Primeiro Plano Ouadrienal de Desenvolvimento Económico e Social 1983-1986: A. Relatório da situacáo actual, op cit. page 9. 

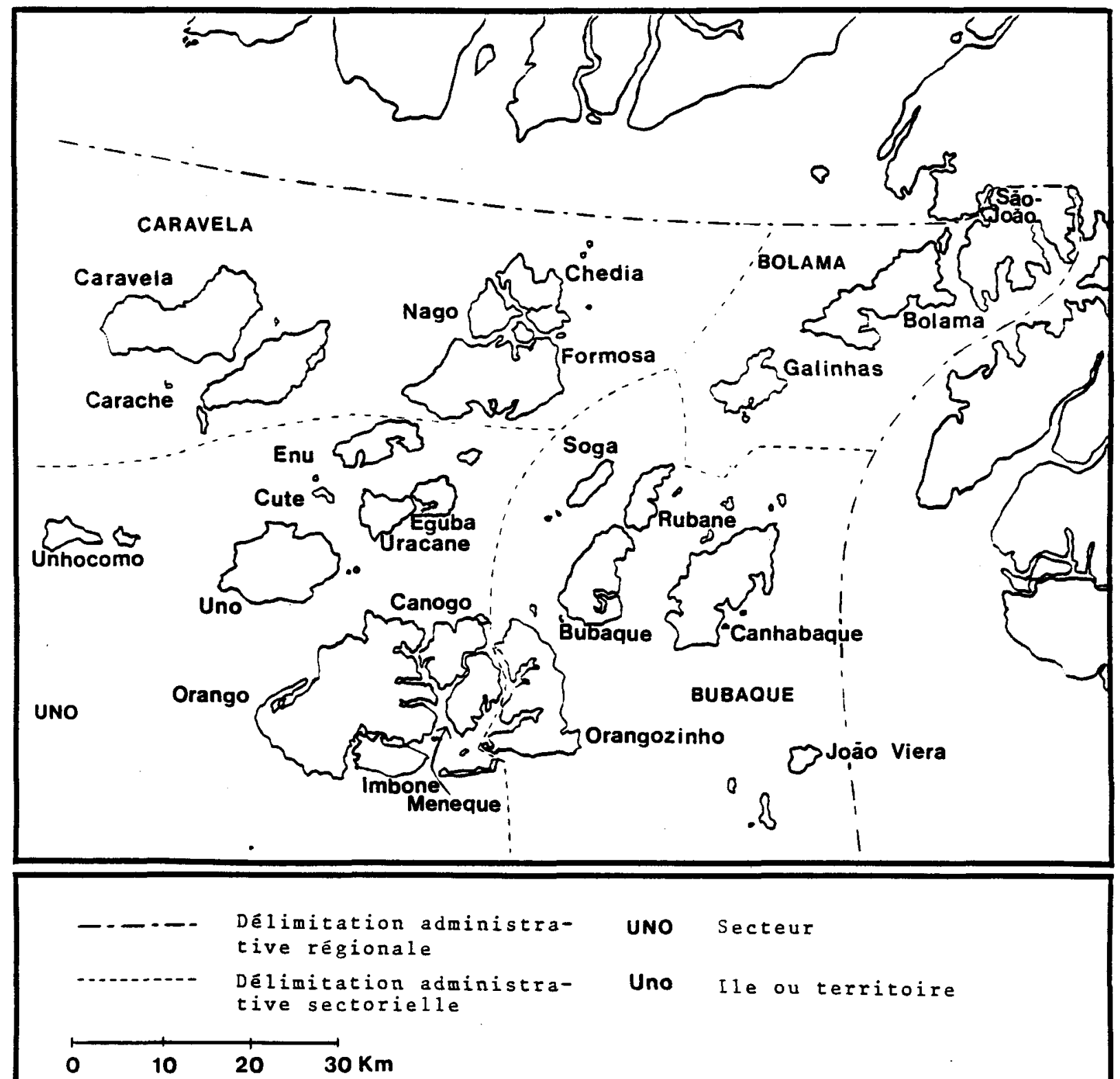

Source: S.C.E.T.-International. Guinée-Bissau, Zone 4: Région de Bolama. 1:500 000. s.1., 1978. Volume III. 
importations, ses principaux fournisseurs étaient le Portugal $(20,1 \%)$, l'Italie $(14,1 \%)$, la Hollande $(9,6 \%)$, la Thailande $(6,8 \%)$, la Suède $(6,2 \%)$ et le Sénégal $(4,6 \%){ }^{1}$

La structure des importations et des exportations du pays concorde avec le modèle général des pays sous-développés où l'on exporte des produits peu ou pas finis pour lesquels on jouit d'un avantage certain et, à l'opposé, on importe des produits de consommation courante. A ce niveau, le pays reste entièrement dépendant de son marché et de ses fournisseurs. ${ }^{2}$ Ainsi s'affirme la contradiction entre les plans du gouvernement bissauguinéen en matière d'autosuffisance alimentaire 3 et le manque de devises étrangères nécessaires à combler ses besoins de consommation.

\subsection{La région Bolama-Bijagos}

\subsubsection{Aperçu général}

La région Bolama-Bijagos s'étend sur une superficie de $1546,43 \mathrm{~km}^{2}$ et comprend l'archipel Bolama-Bijagos et une partie du continent, la section de São-João (voir carte II). Avec un total de 87 îles dont une vingtaine est habitée, la région compte, selon le recensement de 1979, 26598 habitants. Faiblement peuplée, la densité de sa population se compare à celle de la Guinée-Bissau qui est de 21,5 habitant $/ \mathrm{km}^{2}$ et, si l'on se réfère à l'importante surface qu'occupent les mangroves $\left(476,97 \mathrm{~km}^{2}\right)$, la densité de la population passe de 17,2

1 Compilation effectuée à partir de: International Monetary Fund, Yearbook 1988, Direction of Trade Statistics, Washington D.C., 1988, page 209.

2 Pour plus ample information sur cette forme de dépendance, on peut se référer à Samir Amin, Le développement inégal: essai sur les formes sociales du capitalisme périohérique, op. cit, pages 172-176.

3 Nous traiterons plus en détail de la recherche de l'autosuffisance alimentaire dans le cinquième chapitre sur le modèle de développement bissau-guinéen. 
habitants $/ \mathrm{km}^{2}$ à 25 habitants $/ \mathrm{km}^{2} .{ }^{1} \mathrm{Du}$ point de vue administratif, la région est divisée en quatre secteurs ayant chacun ses structures administratives à l'image de la région: Caravela (Formosa), Bubaque, Uno et Bolama.

Cette population est répartie dans 196 villages dont la majorité a moins de 100 habitants. ${ }^{2}$ Seules les villes de Bolama et de Bubaque comptent plus de 1000 habitants soit, suivant le recensement de 1979, 3171 habitants pour Bolama et 1244 pour Bubaque. ${ }^{3}$

La région connaît sept mois de saison sèche et cinq mois de saison des pluies avec une humidité relative de l'air toujours supérieure à $50 \%$ et une température moyenne de $26,9^{\circ} \mathrm{C}$. Avec un écart annuel de seulement $2,6^{\circ} \mathrm{C}$, la région jouit d'un climat de type "tropical pluvieux de savane" où plus de $2000 \mathrm{~mm}$ de pluie tombent annuellement. 4

L'agriculture est la principale activité pratiquée en région. La concentration et l'irrégularité des précipitations (surtout depuis quelques années) qui lessivent et érodent facilement le sol (en plus de ne permettre que difficilement l'approvisionnement de la nappe phréatique), la pauvreté des sols utilisés pour la riziculture et une végétation luxuriante et un climat humide propices au foisonnement de prédateurs de toutes sortes (oiseaux, singes, serpents, termites, gazelles, rats de Gambie, etc.), constituent les principales contraintes avec lesquelles la population doit composer. ${ }^{5} \mathrm{La}$ pratique de méthodes de culture sur brûlis fournit en outre une pression supplémentaire au milieu et la nécessité de permettre au sol de retrouver régulièrement son couvert végétal doit s'accommoder d'une densité de population de 25 habitants $/ \mathrm{km}^{2}$, ce qui, comme le souligne l'étude S.U.C.O.-C.R.A.D., "constitue un taux

1 S.U.C.O.-C.R.A.D. Le développement rural intégré de la région de Bolama. Volume II, op. cit, pages 4-7.

2 Ibid, page 4.

3 Ministerio da Coordenação Económica e Plano, Recenseamento geral da populacão e da habitacão, 16 de abril de 1979. resultados provisórios (fase II), op. cit., pages 41-42.

4 S.U.C.O.-C.R.A.D., op.cit., pages 19-20.

5 Ibid., pages 37-38. 
d'occupation important pour une population qui pratique une agriculture itinérante sur brûlis". 1

La région Bolama-Bijagos présente une originalité particulière puisqu'elle est constituée de trois zones distinctes du point de vue ethnique et socio-économique, et de deux zones au plan géographique. La région englobe ainsi une partie continentale d'où proviennent la majorité des Beafadas qui la peuplent, et une zone insulaire. Dans cette dernière zone, on doit faire la distinction entre l'île de Bolama, habitée pour une bonne partie, de gens nonoriginaires de la région, et le reste de l'archipel dont la population est constituée presqu'exclusivement par l'ethnie bijago, ${ }^{2}$ à raison de $61,13 \%$ de sa population totale ${ }^{3}$ (voir le Tableau 2.6). En fait, si l'on exclut l'île de Bolama et la section de São-João de la population de l'archipel, la population bijago de l'archipel correspond à plus de $80 \%$ de l'ensemble. ${ }^{4}$

\subsubsection{La société bijago}

La société bijago repose sur trois éléments clés: la famille (en particulier le clan matrilinéaire), la classe d'âge et la religion (animisme).

La hiérarchie sociale s'effectue en fonction de classes d'âge où l'on passe de l'une à l'autre par des rites d'initiation dont les modalités, particulièrement en ce qui a trait aux femmes, demeurent assez secrètes. Cette séparation hiérarchique en classes d'âge, qui a la particularité de libérer les hommes adultes de certaines tâches domestiques, s'explique,

\footnotetext{
1 Ibid., page 7.

2 Ministerio da Coordenação Económica e Plano, op. cit., page 136.

3 Ibid., page 133.

4 Ibidem, compilation interne.
} 
paraît-il, par le fait que la société bijago était une société basée sur la guerre et le pillage. Cette stratification permettait aux hommes, dit-on, de se livrer pleinement aux activités guerrières. ${ }^{1}$ Du point de vue économique, notons que la société bijago vit sous un système d'économie de subsistance, dont les caractéristiques principales sont les suivantes:

- inexistence du concept patron/subalterne;

- faible niveau de connaissances techniques et pauvreté en biens d'équipement (notamment au niveau agricole);

- société paysanne où la chasse est marginale au profit de l'agriculture et de la consommation du riz agrémentée de quelques produits liés à la cueillette et au jardinage, et où on ne consomme les animaux domestiques que lors des fêtes et des cérémonies d'ailleurs fort nombreuses. ${ }^{2}$

Un des aspects fondamentaux de la société bijago concerne son égalitarisme économique:

"L'égalitarisme dans l'appropriation des biens est une donnée essentielle dans la société Bijagos. Quiconque affiche une supériorité économique devient dangereux. On s'attend donc à ce qu'un membre qui devient plus riche partage ce surplus de richesse avec les plus nécessiteux, ce qui explique que l'état de fortune individuelle est conservé le plus possible secret, d'autant plus qu'une personne trop fortunée risque d'être accusée de sorcellerie". ${ }^{3}$

1 Christine Henry, Hommes seuls et femmes volages. note sur le mariage des Bijagos de Caravela, texte nonpublié, s.1., juin 1984, 28 pages.

2 Pour mieux connaître la société bijago, on peut se référer à:

Marthe Côté, André Demers, Repères pour un développement nural intégré dans la région de Bolama. Guinée-Bissau, Université Laval, Maîtrise en Aménagement du Territoire et en Développement Régional, novembre 1982, 284 pages;

Danielle Gallois Duquette, Dynamique de l'art bidjoge (Guinée-Bissau): contribution à une anthropologie de l'art des sociétés africaines, Lisbonne, Instituto de Investigaçăo Científica Tropical, 1983, 261 pages;

A. J. Santos Lima, Organizacão económica e social dos Bijagos. Lisboa, Centro de Estudos da Guiné Portuguesa, No. 2, Soc. Ind. de Tipografia, 1947, 143 pages; et,

A. J. Santos Lima, "Os Bijagos e o regime do matriarcado", Boletim Cultural da Guiné Portuguesa. Lisboa, vol. II, No. 7, julho, 1947, pages 593-617.

3 Marthe Côté, André Demers, op.cit., page 53. 
Si l'on excepte l'île de Bolama et la zone continentale de São-João, l'archipel, comme nous l'avons précédemment noté, est peuplé en grande partie et presqu'exclusivement de Bijagos. On peut constater, à partir des déplacements saisonniers liés à l'agriculture et des flux traditionnels d'échange, que le véritable pôle régional naturel semble être Bubaque plutôt que le centre administratif régional, Bolama. Côté et Demers ont remarqué l'importance des flux entre les îles bijagos, et Bolama en est presqu'exclue. Ces flux découlent surtout des migrations dans la culture du riz des Bijagos. Hormis cela, les migrations entre le continent (région du Biombo, au nord-est de la région) et les îles, sont l'oeuvre de l'ethnie pepel, et ont pour cadre l'exploitation du palmier à huile. Les seuls courants vers Bolama viennent des déplacements bijagos de Canhabaque pour la récolte des fruits du palmier. ${ }^{1}$

Tout ceci pose une entrave de taille aux activités régulières des différentes délégations ministérielles régionales (qui ont leur siège à Bolama) et, de manière plus générale, à tout effort de développement régional à partir de Bolama. A preuve, les liaisons maritimes officielles entre la ville de Bolama et le reste de l'archipel ne peuvent s'effectuer que via Bissau. En ce sens, Bubaque est plus près de Bissau que de Bolama. ${ }^{2}$ Aussi n'est-il pas étonnant de constater que sur les neuf projets de coopération de la région, la majorité soit basée à Bubaque plutôt qu'à Bolama:

Projets basés à Bubaque:

- P.N.U.D.3: P.D.I.I.B. (Projecto de Desenvolvimento Integrado das Ilhas Bijagos), O.N.Ụ.;

\footnotetext{
1 Ibid., page 102.

2 Nous parlons ici du transport officiel, c'est-à-dire du réseau de transport national. On omet toutes les petites embarcations de pêche (pirogues) et les bateaux des différents projets qui peuvent se déplacer indépendamment entre les îles. De même, comme nous le verrons ultérieurement, le village de Bolama de Baixo, village bijago du sud-ouest de lîle de Bolama, est traditionnellement lié aux autres îles de l'archipel.

3 P.N.U.D.: Projets des Nations-Unies pour le Développement.
} 
- MANITESE: soins de santé de base et agriculture, Italie;

- PESCARTE: appui à la pêche, Suède;

- MISSION CATHOLIQUE: agriculture, Italie;

- MISSION EVANGELIQUE: agriculture.

Projets basés à Bolama:

- C.E.C.I.-M.D.R.: Projet de Développement Agricole Bolama-Bijagos, Canada;

- C.E.C.I.-D.P.R.: appui à la Délégation Régionale du Ministère du Plan, Canada;

- ILES DE PAIX: construction de bateaux de pèche, Belgique; et,

- PESCARTE: pêche, Italie (Ittica Valdagri).

Une analyse plus poussée nous démontre que des cinq projets basés à Bubaque, tous ont une certaine envergure régionale. Sur les quatre de Bolama, deux s'adressent directement à l'île de Bolama et les deux autres à l'ensemble de l'archipel. Suivant un certain hasard, ces deux derniers projets relèvent du même organisme et le choix de leur emplacement repose sur le fait que les sièges de la Délégation Régionale du Plan et de la Délégation Régionale du M.D.R. ("Ministério do Desenvolvimento Rural", Ministère du Développement Rural) se situent à Bolama.

Ce bref survol de la Guinée-Bissau, de son peuple et de son histoire, ne s'achève pas avec ce chapitre. Tout au long du mémoire, il faudra faire constamment référence au contexte global de la formation sociale africaine de ce pays afin de bien comprendre la réalité bolamienne. Loin de constituer un résumé des principales caractéristiques du pays et de la région Bolama-Bijagos, ce premier chapitre s'avère simplement être une introduction à notre 
sujet, à laquelle il faudra constamment faire référence et ajouter de nouvelles données au fur et à mesure du développement du mémoire.

De même nous devrons constamment garder à l'esprit l'aspect particulier de l'île de Bolama par rapport à l'ensemble de la région Bolama-Bijagos. Ainsi, il importe de bien saisir comment la spécificité de Bolama et celle de l'archipel peuvent s'opposer et cristalliser sous forme de contrainte majeure au développement harmonieux de la région, l'adoption d'un mode de division administrative qui fait de Bolama la capitale de cette région.

L'objet du mémoire n'est pas de traiter de l'ensemble de la région Bolama-Bijagos, mais de lîle de Bolama et de ses particularités. Nos intérêts se porteront précisément sur celle-ci et le reste de l'archipel n'aura, dans le développement de notre recherche, qu'un rôle accessoire, d'autant plus qu'au niveau bibliographique, de nombreux ouvrages et études traitent de l'archipel bijago alors que bien peu d'écrits, pour ne pas dire pratiquement aucun, ne concernent l'île de Bolama. Il devient alors évident que le lecteur ne saura trouver outre mesure, dans le présent mémoire, d'informations originales sur le reste de l'archipel. 


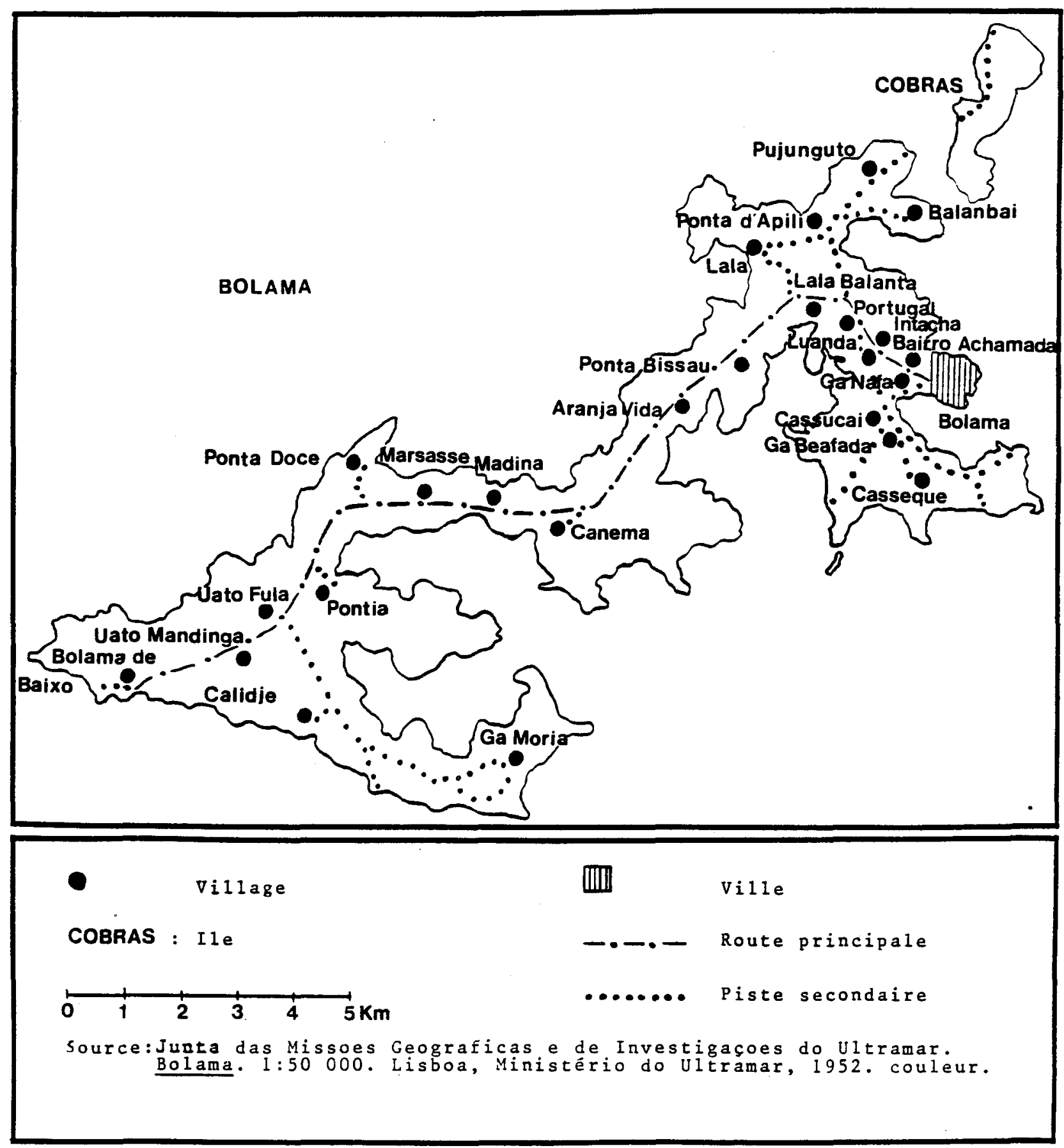




\section{CHAPITRE DEUX}

\section{L'ILE DE BOLAMA, UNE REGION EN SOI}

\subsection{Description physique}

L'île de Bolama, qui constitue le terrain de notre recherche, est la plus près du continent de toutes les îles de l'archipel dont elle n'est séparée que par un étroit canal de quelques deux kilomètres de large. Ile faite sur le sens de la longueur (environ $25 \mathrm{~km}$ par une largeur variant entre 1 et $6 \mathrm{~km}$ ), elle présente un paysage généralement plat (la cote d'altitude la plus forte atteignant 26 mètres) à végétation dense et luxuriante. Une petite île, l'Tle des Cobras, mesurant entre 3 et $4 \mathrm{~km}$ de longueur par un peu plus de $1 \mathrm{~km}$ au plus large, s'étend au nord-est de Bolama. L'étroit canal $(1 \mathrm{~km})$ la séparant de Bolama a la particularité de se retrouver dégagé à marée basse et les habitants peuvent alors le traverser. Nous considérerons, pour les fins du mémoire, que ce territoire et le village qui s'y trouve font partie de Bolama.

Le climat, comme nous l'avons précédemment noté, est caractérisé par la présence de deux saisons. La saison des pluies dure cinq mois à Bolama, de juin à octobre, et la saison sèche, d'une durée de sept mois, s'étend de novembre à mai, deux mois pouvant être considérés comme transitoires entre les saisons. Les précipitations se concentrent plus particulièrement en juillet, août et septembre. Ces trois mois reçoivent en moyenne 78,4\% des précipitations annuelles, alors que la période s'étendant de novembre à mai ne compte, 
en moyenne, que pour $4 \%$ du total des précipitations annuelles. ${ }^{1}$ Le Tableau 2.1 présente les principaux éléments du climat de Bolama selon le mois de l'année.

Depuis les années 1970, on a noté, tout comme pour l'ensemble du pays, une certaine diminution des totaux pluviométriques annuels ${ }^{2}$ (voir Tableau 2.2 et Graphique II). Est-on en présence d'un assèchement du climat de cette partie du globe? Ou encore, cela correspond-t-il à un cycle naturel normal? Dans le cas de Bolama, les plus anciennes données climatiques ne remontent qu'aux années 1920 , ce qui ne nous permet pas de tirer de conclusions précises.

Bien qu'étrangère à l'objet spécifique du mémoire, l'étude du climat s'avère néanmoins indispensable, du moins dans ses grandes lignes. Cette étude constitue en effet un facteur essentiel à la compréhension des modes de vies et de la culture de la population bolamienne. Remarquons ainsi, qu'au niveau de l'agriculture, activité principale des Bolamiens, le climat local n'entraîne pas ou peu de facteurs limitatifs pour les variétés végétales cultivées, tout au plus, comme le souligne l'étude S.U.C.O.-C.R.A.D., "la variation de l'humidité relative lors d'une même journée en saison sèche peut occasionner une légère baisse des rendements même lorsqu'il s'agit de cultures irriguées". 3 Par contre, la concentration de la majeure partie des précipitations sur une période de trois mois provoque, outre une possibilité d'érosion si le sol est laissé à nu et, de toute manière, un lessivage des sols notable lors de fortes pluies (via le ruissellement), une perte importante d'eau, celle-ci ne pouvant pénétrer adéquatement dans le sol. On comprendra de plus que ce phénomène affecte les réserves souterraines d'eau

\footnotetext{
1 S.U.C.O.-C.R.A.D., Le développement rural intéggé de la région de Bolama. République de Guinée-Bissau, Université Laval, Maîtrise en Aménagement du Territoire et Développement Régional, 1982, Volume II, pages 9-10.

2 Diana Lima Handem, Nature et fonctionnement du pouvoir chez les Balanta Brassa. Bissau, I.N.E.P., 1986, page 27.

3 S.U.C.O.-C.R.A.D., op. cit., page 15.
} 


\section{TABLEAU 2.1}

Principales données climatiques

Ile de Bolama

\begin{tabular}{|c|c|c|c|c|c|c|c|c|}
\hline Mois & $\begin{array}{c}\text { Humidite } \\
\text { relative } \\
(1960-1967) \\
(\%)\end{array}$ & $\begin{array}{c}\text { Ensoleillement } \\
\text { relatif } \\
(1960-1978) \\
\text { (heures) }\end{array}$ & $\begin{array}{c}\text { Vitesse du } \\
\text { vent } \\
\text { (1960-1976) } \\
\text { (mètres/ } \\
\text { secondes) }\end{array}$ & $\begin{array}{c}\text { Evaporation } \\
(1941-1979) \\
(\mathrm{mm})\end{array}$ & $\begin{array}{l}\text { Précipitation } \\
\text { (1941-1967 } \\
\text { et 1969-1979) }\end{array}$ & $\begin{array}{c}\text { Température } \\
\text { moyenne } \\
(1969-1979) \\
{ }^{\circ} \mathrm{C}\end{array}$ & $\begin{array}{c}\text { Température } \\
\text { maximale } \\
(1969-1979) \\
{ }^{\circ} \mathrm{C}\end{array}$ & $\begin{array}{c}\text { Temperature } \\
\text { minimale } \\
(1969-1979) \\
{ }^{\circ} \mathrm{C}\end{array}$ \\
\hline $\begin{array}{l}\text { Janvier } \\
\text { Février } \\
\text { Mars } \\
\text { Avril } \\
\text { Mai } \\
\text { Juin } \\
\text { Juillet } \\
\text { Août } \\
\text { Septembre } \\
\text { Octobre } \\
\text { Novembre } \\
\text { Décembre }\end{array}$ & $\begin{array}{l}54,2 \\
61,2 \\
64,2 \\
62,8 \\
64,0 \\
71,7 \\
81,1 \\
86,6 \\
84,6 \\
80,6 \\
70,7 \\
55,4\end{array}$ & $\begin{array}{l}267,8 \\
259,0 \\
291,7 \\
281,7 \\
276,8 \\
179,1 \\
147,6 \\
129,1 \\
159,6 \\
217,2 \\
245,9 \\
255,8\end{array}$ & $\begin{array}{l}2,0 \\
2,4 \\
2,6 \\
3,1 \\
2,9 \\
2,7 \\
2,6 \\
2,7 \\
2,4 \\
1,7 \\
1,8 \\
1,8\end{array}$ & $\begin{array}{r}198,9 \\
179,7 \\
190,2 \\
183,0 \\
169,6 \\
110,2 \\
75,0 \\
58,4 \\
63,3 \\
77,7 \\
105,0 \\
167,7\end{array}$ & $\begin{array}{r}0,72 \\
0,68 \\
0,38 \\
0,25 \\
27,35 \\
211,62 \\
600,29 \\
732,36 \\
437,0 \\
199,89 \\
43,58 \\
4,53\end{array}$ & $\begin{array}{l}25,6 \\
26,6 \\
27,3 \\
27,8 \\
28,2 \\
27,5 \\
26,5 \\
26,1 \\
26,4 \\
27,3 \\
27,5 \\
25,7\end{array}$ & $\begin{array}{l}32,3 \\
33,3 \\
33,7 \\
33,2 \\
33,1 \\
31,5 \\
29,8 \\
29,2 \\
30,0 \\
31,4 \\
32,2 \\
31,9\end{array}$ & $\begin{array}{l}18,9 \\
19,8 \\
20,8 \\
21,8 \\
23,4 \\
23,6 \\
23,2 \\
23,1 \\
22,8 \\
22,2 \\
22,8 \\
19,5\end{array}$ \\
\hline $\begin{array}{c}\text { Total } \\
\text { Moyenne }\end{array}$ & 69,8 & 2711,5 & 2,4 & 1579,8 & 2258,65 & 29,6 & 31,8 & 21,8 \\
\hline
\end{tabular}

Source: S.U.C.O.-C.R.A.D., Le développement rural intégré de la région de Bolama, République de Guinée-Bissau, Volume III, Université Laval, Maîtrise en Aménagement du Territoire et Développement Régional, 1982, page 5. 
TABLEAU 2.2

Pluviométrie annuelle moyenne Ile de Bolama

1924-1987

(en $\mathrm{mm}$ )

\begin{tabular}{|c|c|c|c|c|c|}
\hline Année & Pluie & Année & Pluie & Année & Pluie \\
\hline 1924 & 2289,1 & 1947 & 1813,1 & 1970 & 2149,8 \\
\hline 1925 & 2273,4 & 1948 & 2390,5 & 1971 & \\
\hline 1926 & 2422,5 & 1949 & 2056,9 & 1972 & 1549,6 \\
\hline 1927 & 2156,7 & 1950 & 2526,4 & 1973 & 1698,7 \\
\hline 1928 & 1923,2 & 1951 & 2271,5 & 1974 & 1853,1 \\
\hline 1929 & 2206,6 & 1952 & 2336,5 & 1975 & 2383,4 \\
\hline 1930 & 2194,3 & 1953 & 2100,0 & 1976 & 2940,0 \\
\hline 1931 & 2072,0 & 1954 & 2692,2 & 1977 & 1129,5 \\
\hline 1932 & 2145,8 & 1955 & 2161,6 & 1978 & 2460,6 \\
\hline 1933 & 2650,8 & 1956 & 2310,5 & 1979 & 1635,4 \\
\hline 1934 & 1986,2 & 1957 & 2592,1 & 1980 & 1644,3 \\
\hline 1935 & 1933,3 & 1958 & 3385,8 & 1981 & 2380,5 \\
\hline 1936 & 2906,9 & 1959 & 2508,4 & 1982 & 1838,3 \\
\hline 1937 & & 1960 & 2235,2 & 1983 & \\
\hline 1938 & 2423,6 & 1961 & 211 & 1984 & 1340,2 \\
\hline 1939 & 2109,2 & 1962 & 2416,8 & 1985 & 1692,7 \\
\hline 1940 & 211 & 1963 & 213 & 1986 & 1665,4 \\
\hline 1941 & 1602,5 & 1964 & 2386,0 & 1987 & 1441,5 \\
\hline 1942 & 2035,0 & 1965 & 2591,1 & & \\
\hline 1943 & 2557,9 & 1966 & 2199,2 & Moyenne & \\
\hline 1944 & 2229,3 & 1967 & 2923,8 & annuelle: & $167, \varepsilon$ \\
\hline 1945 & 2492,6 & 1968 & 1508,1 & & \\
\hline 1946 & 2001,9 & 1969 & 2918,2 & Écart-type & 422 \\
\hline
\end{tabular}

Source: Serviço Meteorologico Nacional, Estação Meteorologica de Bolama, Republica da Guiné-Bissau, s.d.. 


\section{GRAPHIQUE II}

Pluviométrie annuelle moyenne

Ile de Bolama

1924-1987

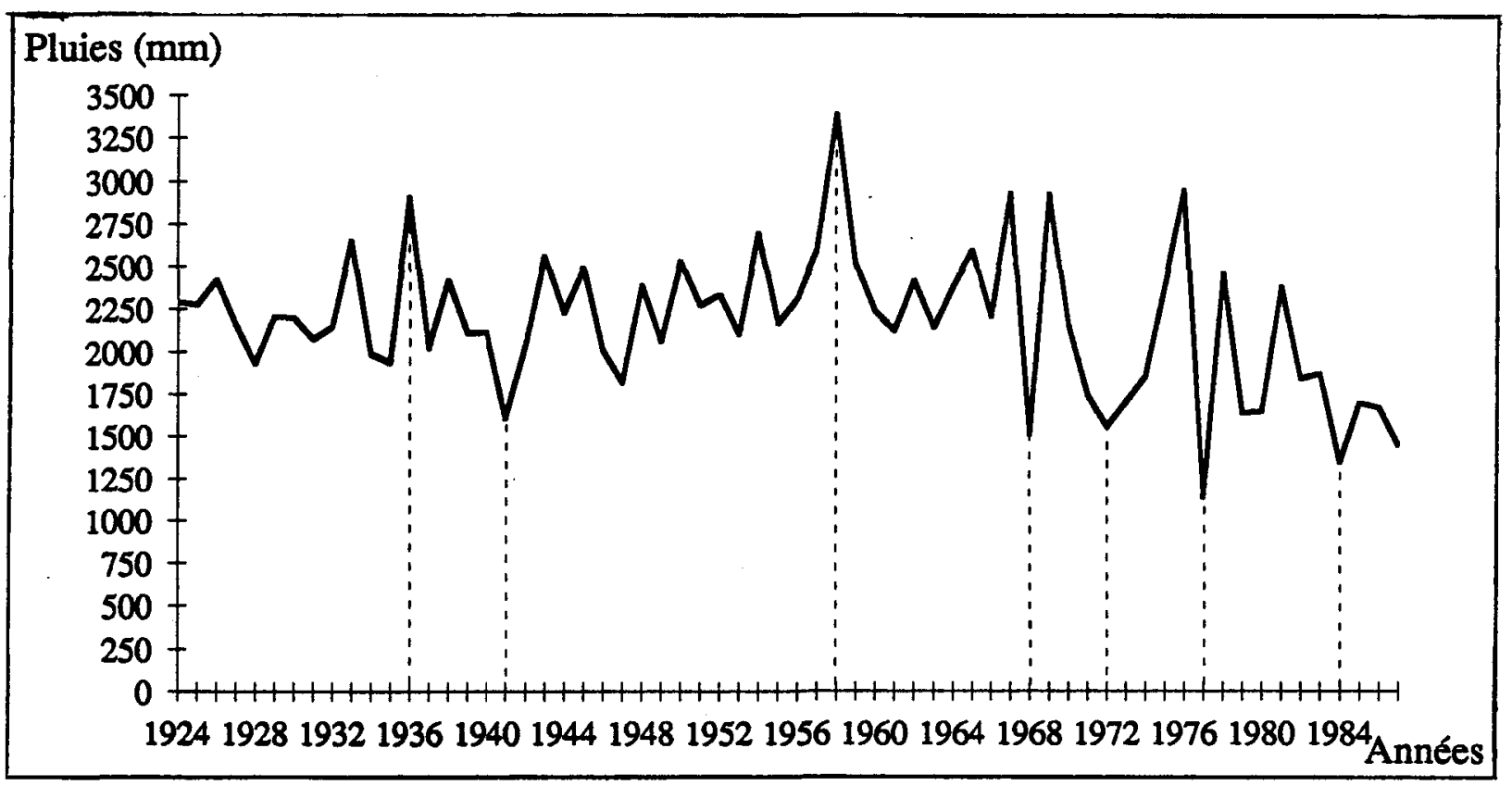

Source: Serviço Meteorologico Nacional, Estação Meteorologica de Bolama, Republica da Guiné-Bissau. 
potable de Bolama. Au fur et à mesure que la saison sèche progresse, ses puits s'assèchent ou se remplissent d'une eau saumâtre ou salée. De plus, on a observé ces dernières années une plus grande variation des précipitations annuelles, de même qu'une irrégularité dans la date de début des pluies. Toutes ces variations climatiques placent le paysan bolamien en situation d'incertitude quant à sa période de semis et au choix des variétés de semences à utiliser. ${ }^{1}$

Du point de vue de l'occupation de ses sols, lî̀le de Bolama s'étend sur une superficie de 8523,7 hectares dont 936 composent l'aire agricole et 7542,7 , l'aire végétale, distribués de la manière indiquée au Tableau 2.3.

Les auteurs de l'étude S.U.C.O.-C.R.A.D. ont défini et calculé les aires d'occupation des sols à partir de photographies aériennes. A l'échelle utilisée (1: 30 000), il leur fut impossible de différencier toutes les espèces d'arbres dans les plantations puisque la superficie qu'occupent ces plantations est trop faible et que tout nettoyage y est inexistant, arbres et buissons de toutes espèces s'y retrouvant en forte densité. Ainsi, seulement 65 hectares ont été identifiés dans toute la région Bolama-Bijagos, et l'on estime à 500 hectares les superficies ne l'ayant pas été. Comme le soulignent les auteurs, il s'agit principalement de plantations de cajous (anacardiers) à Bolama et de palmeraies sur l'île de Bubaque. ${ }^{2}$

L'utilisation du sol sur l'ile de Bolama est beaucoup plus intensive et plus variée que dans les autres îles. Alors que les Bijagos du reste de l'archipel ont une utilisation du sol extensive où $65,8 \%$ de leur occupation agricole est consacrée à la riziculture itinérante sur brûlis et que leur jachère dure de quatre à six ans, la population de Bolama n'utilise que très

1 Ibid., pages 15-16.

2 S.U.C.O.-C.R.A.D., Le développement rural intégré de la région de Bolama. République de Guinée-Bissau, Université Laval, Maîtrise en Aménagement du Territoire et Développement Régional, 1982, Volume III, Carte d'occupation des sols, page 11. 
TABLEAU 2.3

Occupation des sols

De de Bolama

1978

(en hectares)

\begin{tabular}{|c|c|c|c|}
\hline Aire agricole & Superficie & Aire végétale & Superficie \\
\hline \multirow{2}{*}{$\begin{array}{l}\text { Riziculture } \\
\text { sur sol halomorphe } \\
\text { sur sol hydromorphe } \\
\text { sur brûlis }\end{array}$} & \multirow{2}{*}{$\begin{array}{r}296,0 \\
27,9 \\
-\end{array}$} & Forêt sub-humide & - \\
\hline & & \begin{tabular}{|l} 
Palmeraie \\
forte densite \\
moyenne densite
\end{tabular} & $\begin{array}{l}103,9 \\
183,1\end{array}$ \\
\hline Autres cultures & 579,0 & Fourre & 391,8 \\
\hline \multirow{2}{*}{$\begin{array}{l}\text { anacardier } \\
\text { palmier } \\
\text { cocotier } \\
\text { autres fruits }\end{array}$} & \multirow{2}{*}{$\begin{array}{r}34,1 \\
26,2 \\
-\end{array}$} & Savane boisee & 73,8 \\
\hline & & $\begin{array}{l}\text { Savane herbacke } \\
\text { sur sable } \\
\text { lala (sur terrain } \\
\text { inondable) } \\
\text { avec vestiges de } \\
\text { culture }\end{array}$ & $\begin{array}{r}3606,6 \\
405,0 \\
5,0\end{array}$ \\
\hline Ferme d'Etat & 4,7 & Mangrove & 2773,5 \\
\hline Atropont & 5,7 & Habitat & 7,4 \\
\hline Total aire agricole & 946,6 & Total aire végétale & 7550,1 \\
\hline Total identifié & 8496,7 & Total de l'île & 8523,7 \\
\hline
\end{tabular}

Source: S.U.C.O.-C.R.A.D., Le développement rural intégré de la région de

Bolama.République de Guinée-Bissau, Volume III, Carte d'occupation des sols, Université Laval, Maîtrise en Aménagement du Territoire et Développement

Régional, 1982, page 22. 
peu la culture sur brûlis. A Bolama, on pratique une rotation de trois à quatre ans pour les cultures vivrières avec une jachère de trois à cinq ans. La production y est plus diversifiée (céréales, légumineuses, fruits) et la riziculture en eau salée plus développée (25,6\% des sols cultivés). ${ }^{1}$

La différence dans l'utilisation des sols à Bolama par rapport au reste de la région, tient à la spécificité même de l'île, spécificité qui en fait un territoire original, différent du reste de l'archipel à maints égards, comme nous pourrons le constater au fur et à mesure de l'avancement de nos recherches.

"Cette différence dans l'utilisation des sols s'explique par le contexte socioculturel de l'île de Bolama, qui differe de celui de l'archipel sur plusieurs aspects parmi lesquels on peut relever les suivants: 1) la multiplicité ethnique et la diversité des traditions agraires qui en découlent; 2) la présence sur l'île de propriétés étatiques; 3) la densité de la population plus élevée que dans les îles de l'archipel, surtout en regard de la disponibilité des terres; 4) l'urbanisation de l'île et l'implantation d'industries qui commandent une plus forte production de denrées agricoles ainsi qu'une plus grande diversification. Tous ces facteurs sont liés à l'histoire de l'occupation de l'île et du secteur de São-João, par le colonisateur portugais." 2

L'implantation d'industries se doit cependant d'être relativisée car, des deux entreprises construites (fabrique de compote et de jus de fruits et entreprise de tissage de pagnes), une seule aura effectivement fonctionné et ce, de manière sporadique. L'urbanisation de la ville de Bolama, provoquée par l'arrivée de nombreux fonctionnaires et étudiants coupés de la terre, n'aura certes pas été sans conséquence, dont l'apparition d'un marché et l'adoption d'un mode de vie différent de celui du reste de l'archipel (du moins pour les fonctionnaires et les étudiants). Ainsi, au niveau régional global, l'inexistence à toutes fins utiles de villes (sauf

\footnotetext{
1 S.U.C.O.-C.R.A.D., Volume II, op.cit., pages 20-21.

2 S.U.C.O.-C.R.A.D., ibid, page 21
} 
Bubaque) et de marché, est garante, dans une certaine mesure, de la perpétuation de l'utilisation traditionnelle bijago des terres.

2.2 Bolama, d'hier à aujourd'hui

Le peuplement de l'île de Bolama est relativement récent et, jusqu'au XVIII ${ }^{\circ}$ siècle, lîle était inhabitée, si ce n'est par une faune importante, bien que les tribus des territoires voisins y exerçaient certains droits. Le $1^{\circ}$ avril 1752, on y plante le drapeau portugais ${ }^{1}$ sans toutefois s'y établir effectivement. Quarante ans plus tard, en 1792, un Anglais du nom de Philip Beaver conduit une expédition de deux navires et de 270 personnes pour coloniser cette île apparemment riche et généreuse ${ }^{2}$. L'expédition, qui réunissait une poignée de gentlemen, était composée de beaucoup plus de serviteurs, de femmes et d'enfants, que de personnes aptes à faire face à la nature sauvage et aux difficultés d'un tel climat. Avec ses 36 agriculteurs accompagnés de 22 femmes et de 35 enfants, elle s'avéra un échec lamentable, la maladie et les attaques des "sauvages" ayant en moins de deux ans, peu à peu raison des plus entêtés d'entre eux:

"The figures tell their own story: the expedition was hopelessly overweighted with dependents, with gentlemen who clearly did not intend to pursue their pioneering venture too vigorously, and with gentlemen's servants who had no personal stake in the successful colonization of Bulama." 3

1 Fausto Duarte, E assim nasceram cidades e vilas, s.l., Imprensa Nacional da Guiné, 1946, page 42.

2 P. E. H. Hair, "Beaver on Bulama", Boletim Cultural da Guiné Portuguesa, No. 58, an XV, Bissau, 1960, pages 361-371.

3 Ibid, page 371.

"Le portrait (de l'expédition) exprime bien leur histoire: l'expédition était désespérément surchargée de dépendants, avec des gentlemen qui, clairement, n'avaient pas l'intention de poursuivre trop vigoureusement leur aventure de pionnier, et avec leurs serviteurs qui avaient aucune ambition personnelle dans le succès de la colonisation de Bolama" (traduction de l'auteur). 
L'échec de la mission de Beaver est suivi d'une période litigieuse entre l'Angleterre et le Portugal sur l'acquisition et la possession de l'île. En 1830, les Portugais occupent militairement Bolama pour finalement se voir reconnaitre les droits de possession par un arbitrage du président américain Ulysses Grant, le 21 avril $1870 .{ }^{1}$ Lîle se peuplera alors apparaîtront des villages et une petite bourgade, Bolama, qui deviendra la capitale de la colonie jusqu'en décembre $1941 .^{2}$ Les principales dates à retenir concernant la ville de Bolama sont les suivantes: 1882-1883, construction de l'hôpital, 1903, ouverture d'une filiale de la Banco Nacional Ultramarino, 1907-1908, inauguration du réseau de canalisation municipale, 1913, nomination de Bolama au titre de ville, et, 1930, mise en marche de la centrale électrique au mazout. ${ }^{3}$

La ville de Bolama connut, dans la première partie du $\mathrm{XX}^{\circ}$ siècle, son heure de gloire. Les témoignages que nous avons pu recueillir auprès de la population bolamienne font état d'une période d'"abondance", où les marchés et les commerces regorgeaient de marchandises et de nourriture de toutes sortes, où la vie mondaine était animée, où les plages étaient belles et où la Pan American avait même un comptoir en ville... Outre sa vocation de capitale, Bolama et ses environs voyaient l'agriculture se développer et les plantations fruitières abonder. La culture d'arachide aura malheureusement eu un certain effet de dégradation des sols, effet qui, depuis l'abandon de cette culture intensive et la plantation à grande échelle d'anacardiers, a pu, en partie, se résorber. ${ }^{4}$ Bolama perdit cependant son statut de capitale nationale pour se voir attribuer celui de capitale culturelle du pays, ce qu'elle ne saurait véritablement être sans son court passé marqué de la culture lusitanienne. S'ajoute à cela la

\footnotetext{
1 Fausto Duarte, op.cit., pages 42-46.

2 Ibid., page 55.

3 Gabinete do Ministro Residente, Projecto de Câmara Municipal. Bolama, Bolama, Provincia do Sul, março 1988 , pages $3-4$.

4 S.U.C.O.-C.R.A.D., Volume II, op.cit., page 169.
} 
multiplicité ethnique de la colonisation de l'̄̃e, qui ne put qu'ajouter à la vocation culturelle de Bolama, bien que la contribution de chacune de ces ethnies, loin de se matérialiser dans des oeuvres littéraires ou culturelles quelconques, s'évalue plutôt en fonction de l'héritage qu'elles laisseront à un niveau pratique. Côté et Demers remarquent la richesse de cet héritage tout en notant qu'il est unique à Bolama:

"En revanche, lîle de Bolama est habitée par un grand nombre d"ethnies Balantes, Bijagos, Fulas, Mancagnes, Manjaks, etc. - et les Bijagos ne viennent qu'au second rang derrière les Mancagnes. Le savoir traditionnel propre à ces divers groupes et la présence plus intensive de la colonisation portugaise expliquent une plus grande diversité des cultures, des pratiques et techniques utilisées pour l'agriculture et l'élevage. Les Balantes y sont responsables de la connaissance des techniques de production rizicole en "bolanha" d'eau salé; les Nhomincas, de l'introduction de filets de pêche permettant de pêcher de plus gros poissons; les Papeis, d'une exploitation plus intensive de la palmeraie naturelle; les Fulas, d'un élevage contrôlé et de la traite des vaches..." 1

De nos jours, une route de terre de $21 \mathrm{~km}$ de long relie Bolama à l'extrémité sud-ouest de l'île. Praticable en saison sèche, elle est d'accès difficile en saison des pluies. En outre, lîle est criblée de petites pistes non entretenues, souvent inaccessibles en voiture. On note de plus les vestiges d'une piste d'atterrissage abandonnée.

Même si Bolama peut être considérée comme une ville portuaire, l'accès maritime en demeure tout de même assez difficile et restreint, d'une part par une cartographie désuette 2 et, d'autre part, par une liaison maritime insuffisante et souvent inadéquate. ${ }^{3}$ Depuis 1987 ,

1 Marthe Côté, André Demers, Repères pour un développement rural intégré dans la région de Bolama. GuinéeBissau, Université Laval, Mâtrise en Aménagement du Territoire et en Développement Régional, novembre 1982, page 45.

2 Le canal de Bolama compte de nombreux bancs de sable et ceux-ci ne sont ni cartographiés ni balisés. De plus, son accès se limite à la marée haute, faute de dragage. Enfin, notons la présence dans le canal de Bolama, d'un courant variant entre trois et quatre noeuds.

3 Outre les petites embarcations privées (surtout des pirogues), une société privée, la Rodofluvial, qui a pris la relève de la société nationale Guinemar, assure, depuis 1987, la liaison Bissau-Bolama deux fois par semaine, et une fois par semaine vers la région sud du pays, lorsqu'aucune avarie ou pénurie ne sévit. 
l'acquisition d'un traversier d'une capacité d'une dizaine de tonnes ${ }^{1}$ permet d'assurer la liaison avec le secteur de São-João.

La ville se divise en deux parties principales, la ville elle-même et ses quartiers. L'ancienne ville (la "cidade") est composée d'édifices et de maisons de béton datant dans la plupart des cas de l'époque coloniale. Les rues sont parfois bordées de trottoir et la ville, dans son ensemble, si l'on fait fi de son aspect délabré (nombreux bâtiments en ruine totale ou partielle, absence de peinture sur les murs, etc.), est très coquette avec ses édifíces anciens, ses nombreux parcs et son architecture typiquement portugaise où se retrouvent même parfois d'intéressants azulejos. La ville est entourée de quartiers, les "bairros" (N'Tatcha, Assomada, Castelo, São-Jose), où s'alignent des cases africaines typiquement bissau-guinéennes. Ces habitations sont fabriquées à partir de briques de terre, avec plancher en terre battue ou parfois en béton. De forme rectangulaire, elles peuvent avoir une dizaine de mètres de longueur et logent souvent plus d'une famille. Le toit est recouvert de paille ou, chez les plus fortunés, de feuilles de zinc. Ces quartiers périphériques ressemblent aux villages en brousse, chaque famille y ayant ses animaux de basse-cour (poules, canards, chèvres, porcs et plus rarement, moutons) et un petit jardin à l'occasion.

L'alimentation de la ville en électricité est assurée par une centrale datant d'une cinquantaine d'années. L'électricité n'est offerte que le soir, de la tombée du jour (variant entre 18 heures 30 et 19 heures 30 ) à 22 ou 23 heures; le samedi soir, ou encore, les jours de fête populaire, le service est souvent prolongé plus tard que minuit. La centrale fonctionne assez régulièrement quoique les pénuries de combustible et les bris mécaniques peuvent en affecter le service durant des jours, des semaines, voire des mois. Le service électrique

1 Paul Rouillard, Refflexions sur le projet Bolama-Bijagos, Bolama, Projet de Développement Agricole Bolama-Bijagos, C.E.C.I., août 1987, page 14. 
n'atteint pas la plupart des quartiers périphériques et, son coût élevé, fait qu'il n'est abordable que pour les familles qui disposent de revenus monétaires décents.

Le service d'aqueduc est lui-aussi un bien de luxe, et le réseau ne dessert que la ville. La distribution d'eau est soumise aux aléas du climat et de l'approvisionnement en combustible (pour faire fonctionner la pompe du château d'eau) et en pièces. Vers la fin de la saison des pluies, lorsque la nappe souterraine d'eau douce est bien remplie, l'approvisionnement est régulier, soit plusieurs heures par jour. Très tôt après la fin des pluies, le service n'est assuré que quelques heures quotidiennement, deux ou trois heures, pour ensuite, plus tard en saison, être réduit en temps (moins d'une heure) et en fréquence (à tous les deux jours). A la fin de la saison sèche et au début de la saison des pluies, le service se fait encore plus rare. Evidemment, en cas de bris majeur, la distribution d'eau peut être paralysée pendant de nombreux jours.

Aux graves lacunes du réseau d'aqueduc (pénuries quotidiennes, taille limitée du réseau, coût élevé de l'eau), il convient d'ajouter que sa gérance, tout comme celle du service électrique, assurée par le Comité de Secteur de Bolama, implique certaines pratiques de favoritisme dans la distribution à de nouveaux abonnés. De ce fait, de nombreuses habitations sont équipées de puits. Dans les quartiers périphériques, et dans la plupart des villages, des puits fermés, avec pompe manuelle, viennent d'être installés grâce à la coopération hollandaise.

Les villages de l'île sont composés de cases à toit de paille. La plupart des habitations de ces villages sont regroupées en un ou plusieurs ensembles, bien que dans certains villages, elles soient dispersées. Parfois encore, dans un même village, les cases sont rassemblées en plusieurs endroits selon l'ethnie ou la famille. Aucun des villages de Bolama n'a d'électricité, 
ni d'eau courante. L'étude S.U.C.O.-C.R.A.D. identifia 48 villages sur les îles de Bolama et des Cobras, ${ }^{1}$ et le recensement général, 41 (comme on peut le voir au Tableau 2.4). On peut facilement expliquer cette différence par le fait que parfois, ces villages sont si petits qu'ils se regroupent sous le même nom, ou encore sous les mêmes chefs, le même comité de base, selon l'ethnie et le cas.

Il importe à ce stade-ci de préciser que l'ensemble des habitants des villages, hormis ceux des villages situés à la périphérie de la ville, s'adonnent aux activités traditionnelles (agriculture, pêche, cueillette, etc.). Les seules exceptions concernent quelques commerçants et fonctionnaires en poste dans certains villages pour assurer les services de santé, de police et d'éducation. La ville de Bolama regroupe pour sa part les activités reliées à la fonction publique, au commerce et au secteur manufacturier tout modeste soit-il. L'île, comme nous l'avons déjà noté, forme une société duale où deux modes de vie se rencontrent, l'un traditionnel, concentré en grande partie à la campagne, et l'autre, plus moderne, à la ville.

\subsection{La population}

Les données statistiques en Guinée-Bissau sont pour le moins ambiguës et il est rare de rencontrer diverses sources présentant les mêmes résultats. Outre l'emploi de méthodologies souvent différentes, ces variations peuvent s'expliquer en partie par le recours massif à l'estimation ou encore, dans le cas d'études sur la population, par la mentalité même des populations consultées qui, peu habituées à être interrogées, orientent parfois leurs réponses en fonction de ce qu'ils pensent pouvoir faire plaisir à l'enquêteur. Lors d'une enquête auprès d'un échantillon de productrices de légumes à Bolama, on aura d'ailleurs noté que les

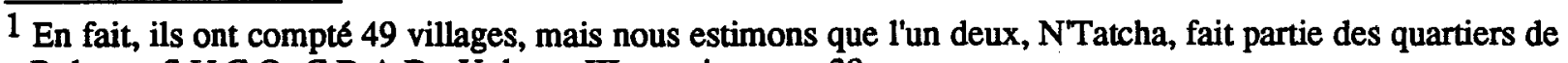
Bolama: S.U.C.O.-C.R.A.D., Volume III, op.cit., page 39. 
maraîchères avaient tendance à surestimer grandement leur production et qu'elles associaient cet exercice d'évaluation de la production à un "concours de productivité", malgré les efforts répétés de persuasion de l'équipe d'enquêteurs. ${ }^{1}$

Dans plusieurs cas, tout au long du mémoire, aurons-nous l'occasion de constater que l'absence de système de compilation et de cueillette de données en Guinée-Bissau contraint les autorités à recueillir, diffuser et à se fier à des information hypothétiques. Remarquons ainsi le recours à l'estimation pour les données sur la production agricole par exemple, que l'on recueille auprès des acteurs concernés, Ministère du Développement Rural, Service Météorologique National, responsables politiques locaux, paysans, etc., 2 ou encore la pratique à l'approximation, lors des calculs relatifs aux principaux indicateurs économiques nationaux où l'on doit considérer les secteurs monétaires et non-monétaires. ${ }^{3}$

Pour l'île de Bolama, comme pour l'ensemble du pays, nous disposons de deux sources de données en matière de population:

- le recensement national d'avril 1979, effectué sous la supervision d'un expert des Nations-Unies; et,

- le recensement annuel pour fins d'impôt qu'effectuent les comités de secteur auprès des comités de tabanca (village).

Les résultats des deux sources pour Bolama ne concordent pas, le recensement de 1979 attribue 6815 habitants à l'île alors que celui du comité de secteur, effectué moins d'un an

1 Brigitte Boies, Alain Rousseau. Enquête sur la commercialisation des produits maraîchers. Bolama, Projet de Développement Agricole Bolama-Bijagos, C.E.C.I., mai 1988. page 29.

2 Ministère du Plan, Estimation du déficit céréalier en 1988 et requête d'assistance introduite auprès de la communauté internationale, Bissau, décembre 1987, page 1.

3 Groupement Lavalin/Delcanda, Etude générale sur les transports, s.1., Banque Africaine de Développement, juin 1980, volume I, Base socio-économique, page 2-17. 
plus tard, ne fait état que de 4366 habitants. ${ }^{1}$ La différence entre ces deux sources est très importante et certains auteurs consultés n'hésitent pas à accuser la méthodologie pour expliquer cet écart. ${ }^{2}$ En effet, le recensement de 1979 , qui a bénéficié du concours du F.N.U.A.P. (Fonds des Nations-Unies pour les Activités en matière de Population) et du P.N.U.D. (Programme des Nations-Unies pour le Développement) ${ }^{3}$ et qui a eu liei du 16 au 30 avril 19794 , comptabilisait la population de fait ("de facto") et celle de droit ("de jure"). On sait que la population de fait se compose de toutes les personnes se trouvant effectivement sur le lieu de recensement lors de la visite du recenseur alors que celle de droit concerne l'ensemble des personnes y résidant, qu'elles soient présentes ou non lors du recensement. Ceci entraîne inévitablement une surestimation de la population totale, une partie de celle-ci étant comptabilisée deux fois. De ce fait, les auteurs consultés se fient-ils plus aux résultats des comités de secteur qu'à ceux du recensement, ne serait-ce que par leur caractère plus récent.

Pour notre part, nous doutons que plus de 35\% de la population recensée sur l'île de Bolama se trouvait en situation de migration temporaire ou encore était décédée pendant la période séparant les deux relevés. Sur l'avis de nombreux fonctionnaires du gouvernement (Ministère du Plan et Comité d'Etat), nous avions conclu, lors de notre séjour au pays, à une sous-estimation de la population par les enquêtes des comités de secteur. En effet, celles-ci ayant lieu dans le but principal d'établir les listes d'imposition, la mentalité villageoise

\footnotetext{
1 Marthe Côté, André Demers, op. cit., page 44.

2 Ibid. page 43; et, S.U.C.O.-C.R.A.D., Volume II, op. cit., page 30.

3 Ministerio da Coordenação Economica e Plano, Recenseamento Geral da Populacao e Habitaçao. 16 de abril de 1979: resultados provisorios (fase II), Bissau, Departamento Central de Recenseamento, fevereiro 1982, page II.

4 Ministério da Coordenação Económica e Plano, Recenseamento geral a população e da habitação. 16 de abril 1979. Bissau, Departamento Central de Recenseamento, fevereiro 1982, Volume II, Lista nominativa das localidades recenseadas.
} 
particulière de toute communauté vivant en quelque sorte en repli sur soi, nous aura porté a supposer que les comités de villages, responsables dans leurs villages respectifs de préparer la liste de leurs villageois pour la remettre aux responsables des comités de secteur, feront bien souvent tout leur possible pour soulager leurs concitoyens d'un fardeau fiscal qui leur apporte en contrepartie bien peu. L'enquête de tabanca nous aura par ailleurs permis de voir certains villages où la population avait assurément été diminuée de moitié par rapport aux résultats officiels du recensement de 1979 et où l'on prétendait qu'il y avait moins d'habitants qu'il n'y a effectivement de cases (habitations). Comme le démontre le Tableau 2.4, la différence entre les résultats du recensement de 1979 et ceux du dernier recensement de secteur disponible (1987) atteint presque 30\%. Cet écart peut-il être dû uniquement à une rébellion contre limpôt et à une surestimation de la population de la part des recenseurs de 1979? Hans Geisslhofer tient peut-être une partie de l'explication lorsqu'il souligne:

"Mais, sept ans après l'indépendance, on doit constater que l'exode rural dans ce petit pays est encore plus accéléré que dans des pays voisins, malgré toute la volonté du Parti de donner, dans le développement, la priorité à l'agriculture, maintes fois exprimée dans les différentes déclarations officielles. Environ 45\% des investissements jusqu'en 1979 ont été absorbés par l'infrastructure urbaine et inter-urbaine." 1

L'émigration des populations rurales et de celles des petites villes vers Bissau et vers d'autres villes africaines (en particulier Dakar) est une réalité bissau-guinéenne qui affecte pratiquement toutes les familles. Cet exode touche aussi l'ile de Bolama ${ }^{2}$ et se concrétise par l'absence d'hommes dans certains villages.

1 Hans Geisslhofer, Planification villageoise en Guiné-Bissau; problèmes-expériences-perspectives, Dakar, E.N.D.A., Série Etudes et Recherches, No. 70-81, octobre 1981, page 4.

2 Yves Brunet, Nancy Thède, Rapport de mission d'étude sur le projet d'étude pour la mise en place de structures régionales de développement (Guinée-Bissau), Montréal, le 5-7-79, page 6. 
TABLEAU 2.4

Population totale par village

Ile de Bolama

1979 et 1987

\begin{tabular}{|c|c|c|c|c|c|c|}
\hline \multirow{2}{*}{$\begin{array}{c}\text { Ville } \\
\text { Villoge }\end{array}$} & \multicolumn{3}{|c|}{ Recensement de 1979(1) } & \multicolumn{3}{|c|}{ Résultats de 1987(2) } \\
\hline & Honmea & Femmes & Total & Hommes & Femmes & Total \\
\hline 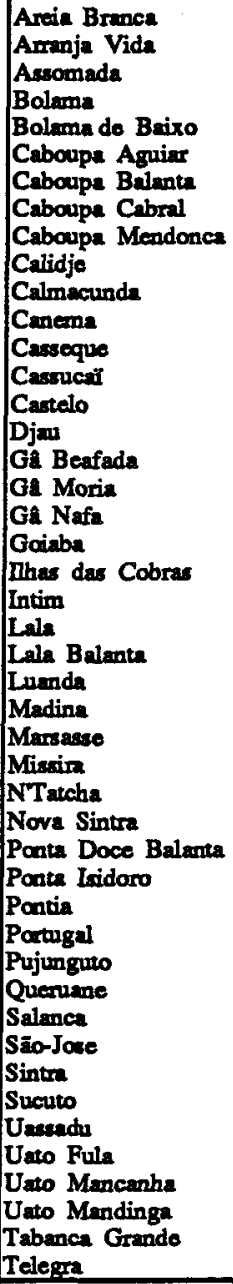 & $\begin{array}{r}9 \\
34 \\
- \\
1673 \\
53 \\
8 \\
18 \\
29 \\
22 \\
55 \\
7 \\
45 \\
66 \\
132 \\
- \\
11 \\
146 \\
86 \\
30 \\
16 \\
30 \\
12 \\
28 \\
15 \\
139 \\
54 \\
31 \\
7 \\
- \\
16 \\
38 \\
5 \\
17 \\
93 \\
18 \\
137 \\
5 \\
45 \\
20 \\
25 \\
21 \\
57 \\
28 \\
75 \\
\end{array}$ & $\begin{array}{r}6 \\
34 \\
1498 \\
50 \\
4 \\
25 \\
29 \\
26 \\
58 \\
16 \\
52 \\
83 \\
139 \\
- \\
10 \\
181 \\
72 \\
42 \\
12 \\
26 \\
10 \\
41 \\
17 \\
138 \\
45 \\
31 \\
18 \\
- \\
20 \\
35 \\
3 \\
20 \\
92 \\
23 \\
121 \\
10 \\
50 \\
35 \\
36 \\
55 \\
24 \\
\end{array}$ & $\begin{array}{r}15 \\
68 \\
- \\
3171 \\
103 \\
12 \\
43 \\
58 \\
48 \\
113 \\
23 \\
97 \\
149 \\
271 \\
- \\
21 \\
327 \\
158 \\
72 \\
28 \\
56 \\
22 \\
69 \\
32 \\
277 \\
99 \\
62 \\
25 \\
- \\
36 \\
73 \\
8 \\
37 \\
185 \\
41 \\
258 \\
15 \\
102 \\
157 \\
52 \\
57 \\
52 \\
\end{array}$ & 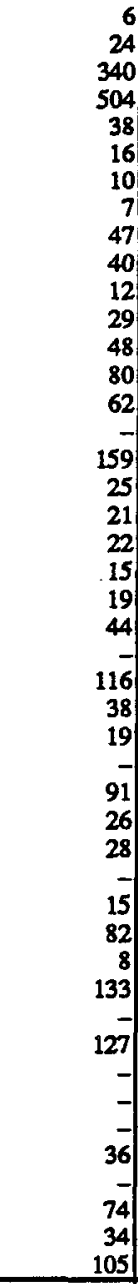 & $\begin{array}{r}6 \\
31 \\
470 \\
621 \\
43 \\
23 \\
8 \\
13 \\
43 \\
44 \\
21 \\
36 \\
86 \\
116 \\
75 \\
- \\
200 \\
24 \\
55 \\
29 \\
14 \\
17 \\
39 \\
130 \\
40 \\
14 \\
-144 \\
36 \\
21 \\
24 \\
88 \\
135 \\
55 \\
140 \\
5 \\
\end{array}$ & $\begin{array}{r}12 \\
55 \\
810 \\
1125 \\
81 \\
39 \\
18 \\
20 \\
90 \\
84 \\
33 \\
65 \\
134 \\
196 \\
137 \\
- \\
359 \\
49 \\
76 \\
51 \\
29 \\
36 \\
83 \\
246 \\
248 \\
78 \\
33 \\
- \\
235 \\
62 \\
49 \\
263 \\
39 \\
170 \\
16 \\
268 \\
- \\
267 \\
- \\
- \\
73 \\
-\end{array}$ \\
\hline Total & 3462 & 3409 & 6871 & 2500 & 3116 & 5616 \\
\hline
\end{tabular}

(1): Ministério da Coordenação Económica e Plano, Recenseamento Geral da População e Habitacão, 16 de abril de 1979; resultados provisorios (fase II), Bissau, Departamento Central de Recenseamento, fevereiro 1982, page 41.

(2): Comité de Estado do Sector de Bolama, Recenseamento de 1987, Bolama, 1987. 
Tous ces facteurs nous aurons donc incité, à l'instar de Côté et Demers et de S.U.C.O.C.R.A.D., à favoriser les résultats des recensements sectoriels plutôt que ceux du recensement général de 1979, en prenant compte des remarques suivantes:

- les résultats du recensement général sont trop élevés de par la méthodologie employée; et,

- ceux des comités de secteur sont vraisemblablement légèrement inférieurs à la réalité puisqu'ils servent avant tout à la levée d'impôt.

Il s'en suit que:

- le nombre d'habitants devrait se situer entre ces deux résultats puisqu'il faut tenir compte de l'augmentation naturelle de la population et la perte due a l'exode vers les villes du continent. ${ }^{1}$

La ville de Bolama comptait en 1979, selon le recensement, 3171 habitants. Le comité de secteur évalue ce nombre, pour 1987, à 2574 (aux résultats de la ville de Bolama, il ne faut pas oublier d'ajouter ceux des quartiers de Assomada, Castelo, N'Tatcha et São-Jose). La ville de Bolama est composée pour une bonne part, d'employés de la fonction publique (plus ou moins 715 personnes) et d'étudiants (environ 900) ${ }^{2}$ dont plusieurs n'habitent à Bolama que durant la période scolaire et donc, ne sont pas tous recensés à Bolama. Une enquête en milieu urbain menée en 1988 par le projet Iles de Paix, obtient des résultats semblables aux nôtres en ce qui a trait au total de la population de la ville de Bolama, la chiffrant à 2592 personnes. ${ }^{3}$

1 Alain Rousseau, Notas sobre o inquérito de tabanca para a ilha de Bolama e a seccão de Såo-João, Bolama, D.P.R.-Bolama-Bijagos, outubro de 1987, pages 4-5.

2 Philippe de Braconier, Elizabeth Drory, Rapport de mission de juin 1985: préétude de l'Ile de Paix de Bolama, Huy, Iles de Paix, 1985,page 6.

3 Entrevue auprès de Tina Stegemann, anthropologue, Bolama, lles de Paix, $1^{\circ}$ juin 1988. 
La présence d'un petit centre urbain sur le territoire étudié nous oblige à perpétuellement opérer une distanciation face à une société que nous avons maintes fois qualifiée de rurale. Il est aussi évident qu'on ne puisse non plus aborder la réalité bolamienne avec une grille d'analyse axée sur les phénomènes urbains. La formation sociale bolamienne nous apparaît alors , comme nous l'avons déjà souligné, sous les traits d'une société duale, alignant deux mondes différents, l'un urbain, l'autre rural, qui sont sur de nombreux plans, coupés l'un de l'autre. La spécialisation des habitants de la ville de Bolama dans des activités liées au tertiaire, en fait l'exemple type de la ville déclassée du tiers-monde, plus ou moins coupée du monde rural qui l'entoure, et dépendante de Bissau et de l'aide internationale pour nourrir sa population improductive. Hormis quelques pêcheurs, tailleurs, tisserands, et le travail de jardinage des femmes, la ville n'est que bien peu productive et ses seules richesses apparentes demeurent son histoire, sa vocation culturelle et sa diversité ethnique.

Cette dualité de la formation sociale bolamienne nous poussera à l'aborder par son monde rural, l'avenir du pays reposant avant tout dans un premier temps, à notre avis et aussi de celui des divers intervenants socio-économiques du milieu, dans un développement en accord avec les potentialités naturelles du milieu (agriculture, pêche, artisanat utilitaire). Le monde urbain sera abordé principalement sous un angle lui accordant particulièrement des tâches de mise en place des infrastructures nécessaires au développement du pays (réseaux de distribution, services à la production, formation et éducation) et de développement d'activités de production possibles en milieu urbain (production artisanale de biens de consommation et de production). Loin de vouloir éloigner la ville de nos préoccupations, nous voulons plutôt la voir comme un appui au développement d'un monde qui est avant tout rural et où l'urbain n'a souvent qu'un rôle de parasite lié au tertiaire. Nous aborderons ainsi toujours, lorsque possible, la réalité bolamienne à partir du monde rural, même si la ville fera constamment 
partie de nos préoccupations et sera tout de même toujours l'endroit où sont concentrés les services. Il ne sera donc pas étonnant de constater l'importance que l'on accordera à l'enquête de tabanca, enquête axée sur le monde rural, bien que certains villages peuvent être effectivement considérés comme des quartiers de la ville de Bolama. C'est le cas, par exemple, d'Assomada et de N'Tatcha qui sont des quartiers typiquement urbains ou encore Ga-Nafa, Luanda et Portugal qui, bien que situés à la périphérie de la ville, présentent tous les traits apparents des villages de la campagne.

Cette attitude de relativisation de l'importance du monde urbain dans le développement de Bolama n'étonne guère si l'on considère le caractère artificiel de la ville. L'important phénomène d'exode rural dont nous avons déjà traité peut nous amener (et nous amène effectivement) à redouter un surplus de main-d'oeuvre en milieu urbain, que ce soit à Bolama ou dans d'autres villes du pays. Cette main-d'oeuvre, en quittant le monde rural, le dépouille du même coup de sa force de travail. Ainsi, la population de l'île de Bolama a, en proportion, moins d'hommes que l'ensemble du pays avec $44,64 \%$ d'hommes contre $48,42 \%$ pour la Guinée-Bissau ${ }^{1}$ et assurément beaucoup plus pour la ville de Bissau si la tendance bolamienne prévaut aussi dans les autres régions. Nos intentions ne sont pas là de dénigrer l'importance de la main-d'oeuvre féminine qui, encore plus en Afrique qu'ailleurs, a un rôle de soutien familial, mais plutôt de souligner la nécessité du recours à la main-d'oeuvre masculine dans l'exécution de tâches agricoles essentielles (notamment dans la riziculture), ne serait-ce que compte tenu de la division sexuelle traditionnelle du travail que l'on remarque chez chacune des ethnies du pays. Nous pourrons parallèlement à l'avancement du mémoire, nous familiariser avec ces coutumes traditionnelles, mais il convenait néanmoins à ce stade-ci

1 Comité d'Etat du secteur de Bolama, 1987, compilation interne; et, Encyclopaedia Britannica, 1987. Britannica Book of the Year, Chicago, Encyclopaedia Britannica Inc., page 660. 


\section{GRAPHIQUE III}

Distribution de la population

selon le sexe et l'âge

Ile de Bolama

1987

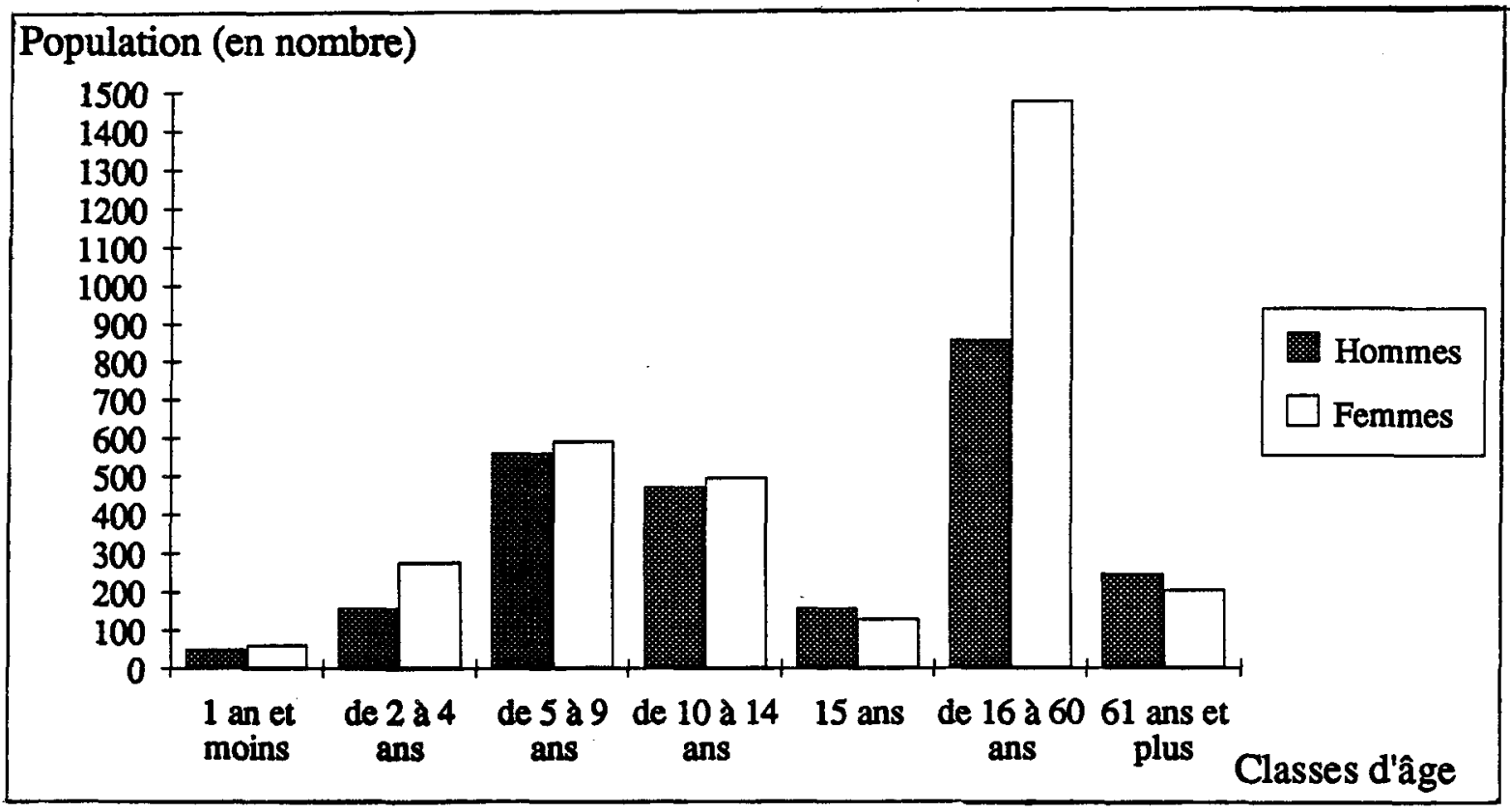

Source: Comité de Estado do Sector de Bolama, Recenseamento de 1987, Bolama, 1987, compilation interne. 
de souligner l'existence de ce fait qui occasionne dans son ensemble, une pénurie de maind'oeuvre masculine dans l'île, comme en fait foi la distribution des sexes pour la catégorie des 16 a 60 ans, illustrée dans le Graphique III.

Le manque d'hommes de cette classe d'âge frappe encore plus durement certains villages qui se voient ainsi privés d'une main-d'oeuvre essentielle à leur développement. Nous traiterons ultérieurement de la division sexuelle du travail à Bolama mais, entre-temps, notons qu'à partir des statistiques de 1987 du comité de secteur sur la population de l'île, nous avons calculé un ratio représentant la part d'hommes âgés entre 16 et 60 ans par rapport au nombre total d'habitants et ce, pour chaque village (voir Tableau 2.5). La moyenne de l'ensemble de l'île est de 0,15. La ville de Bolama affiche 0,16 alors que plusieurs villages sont aux prises avec une pénurie criante d'hommes en âge de travailler: Pontia 0,03 (un homme pour 39 habitants), Caboupa Cabral, 0,05 (un homme pour 20 habitants), Cassucaï, 0,05 (dix hommes pour 116 habitants), Calmacunda, 0,06 (deux hommes pour 33 habitants), Casséqué, 0,06 (8 hommes pour 134 habitants), etc. Ce manque de maind'oeuvre masculine aura maintes fois été soulevé par les responsables des villages lors de l'enquête de tabanca, et par les intervenants en général. Il s'ajoutera évidemment aux différentes contraintes du milieu face à son développement et s'avérera, à nos yeux, être une contrainte de nature conjoncturelle plutôt que structurelle. Ainsi, comme le souligne cet intervenant étranger en poste à Bolama, à propos de ce problème de main-d'oeuvre masculine:

"Le problème est particulièrement aigu en périphérie de Bolama: dans certains villages, en effet, on se demande tout simplement s'il y vit des hommes de la génération des 18-40 ans. Dans d'autres, où le problème est moins critique, l'on en constate pas moins pour autant un taux de masculinité anormalement faible. En fait, sur toute l'île, et encore plus près de la ville où les conditions de vie sont plus difficiles et les traditions moins bien conservées, la tendance à, et l'émigration elle-même, principalement vers la capitale Bissau, de cette catégorie de personnes sont très fortes. L'on peut s'imaginer, par exemple, l'effet de 
TABLEAU 2.5

Distribution de la population selon l'âge et le sexe De de Bolama 1987

\begin{tabular}{|c|c|c|c|c|c|c|c|c|c|c|c|c|c|c|c|c|c|c|}
\hline \multirow{2}{*}{$\begin{array}{c}\text { Vure } \\
\text { Villoge }\end{array}$} & \multicolumn{2}{|c|}{ 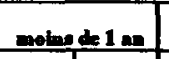 } & \multicolumn{2}{|c|}{ de2+4an } & \multicolumn{2}{|c|}{ destang an } & \multicolumn{2}{|c|}{ de 10 is $14 \mathrm{ems}$} & \multicolumn{2}{|c|}{$15, \mathrm{am}$} & \multicolumn{2}{|c|}{ de 164 ictens } & \multicolumn{2}{|c|}{ o1 am et phe } & \multicolumn{2}{|c|}{ Totel partid } & \multirow{2}{*}{ Trebal } & \multirow{2}{*}{$\begin{array}{c}\text { How } \\
\text { poput } \\
\text { lntion }\end{array}$} \\
\hline & H & P & H & p & н & $\boldsymbol{p}$ & H & $\mathbf{P}$ & H & $\mathbf{P}$ & $\mathbf{H}$ & p & | & $\mathbf{F}$ & H & $\mathbf{F}$ & & \\
\hline 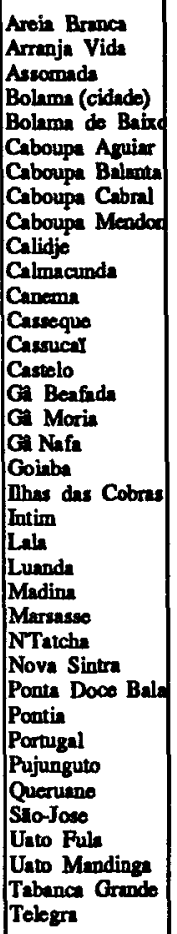 & 23 & 26 & \begin{tabular}{r|r}
2 & 15 \\
6 & \\
2 & \\
4 & \\
2 & \\
1 \\
6 \\
2 \\
5 \\
8 \\
1 \\
3 \\
1 \\
5 \\
2 \\
13 \\
5 \\
6 \\
2 \\
2 \\
4 \\
18 \\
7 \\
1 \\
7 \\
6 \\
12
\end{tabular} & 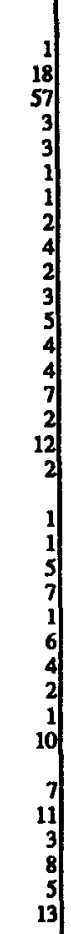 & $\begin{array}{r}1 \\
84 \\
107 \\
9 \\
5 \\
2 \\
9 \\
5 \\
3 \\
7 \\
14 \\
16 \\
15 \\
39 \\
2 \\
6 \\
6 \\
5 \\
15 \\
20 \\
9 \\
4 \\
27 \\
3 \\
3 \\
13 \\
1 \\
42 \\
30 \\
7 \\
9 \\
8 \\
28\end{array}$ & \begin{tabular}{r|}
1 \\
6 \\
93 \\
111 \\
5 \\
2 \\
1 \\
5 \\
11 \\
9 \\
2 \\
8 \\
10 \\
16 \\
20 \\
39 \\
1 \\
12 \\
4 \\
5 \\
8 \\
27 \\
4 \\
23 \\
8 \\
6 \\
9 \\
38 \\
29 \\
13 \\
13 \\
13 \\
32
\end{tabular} & \begin{tabular}{r|}
12 \\
68 \\
71 \\
6 \\
2 \\
4 \\
3 \\
7 \\
11 \\
1 \\
2 \\
9 \\
20 \\
5 \\
28 \\
4 \\
4 \\
5 \\
2 \\
7 \\
8 \\
20 \\
7 \\
4 \\
25 \\
9 \\
2 \\
8 \\
12 \\
24 \\
21 \\
6 \\
14 \\
9 \\
24
\end{tabular} & \begin{tabular}{r|r}
2 \\
61 \\
104 \\
10 \\
1 \\
4 \\
6 \\
2 \\
4 \\
4 \\
10 \\
20 \\
12 \\
37 \\
7 \\
8 \\
2 \\
3 \\
1 \\
5 \\
19 \\
6 \\
28 \\
6 \\
2 \\
4 \\
9 \\
11 \\
20 \\
12 \\
9 \\
20
\end{tabular} & \begin{tabular}{r|}
1 \\
31 \\
13 \\
2 \\
1 \\
3 \\
3 \\
2 \\
4 \\
10 \\
70 \\
10 \\
1 \\
2 \\
1 \\
1 \\
10 \\
1 \\
1 \\
4
\end{tabular} & $\begin{array}{l}1 \\
32 \\
15 \\
2\end{array}$ & \begin{tabular}{r|}
4 \\
7 \\
121 \\
210 \\
12 \\
5 \\
2 \\
1 \\
19 \\
17 \\
2 \\
9 \\
8 \\
10 \\
23 \\
47 \\
12 \\
7 \\
7 \\
4 \\
6 \\
15 \\
32 \\
12 \\
7 \\
17 \\
8 \\
17 \\
1 \\
17 \\
4 \\
40 \\
48 \\
15 \\
36 \\
6 \\
22
\end{tabular} & $\begin{array}{r}3 \\
14 \\
244 \\
275 \\
32 \\
12 \\
5 \\
2 \\
20 \\
24 \\
10 \\
19 \\
48 \\
54 \\
32 \\
96 \\
10 \\
13 \\
17 \\
5 \\
7 \\
24 \\
63 \\
17 \\
7 \\
71 \\
14 \\
17 \\
13 \\
49 \\
7 \\
59 \\
68 \\
14 \\
30 \\
19 \\
68\end{array}$ & 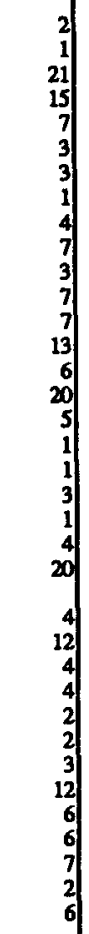 & 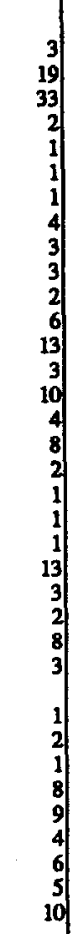 & \begin{tabular}{r|}
24 \\
240 \\
304 \\
38 \\
16 \\
10 \\
7 \\
47 \\
40 \\
12 \\
29 \\
48 \\
80 \\
62 \\
159 \\
25 \\
21 \\
22 \\
15 \\
19 \\
44 \\
116 \\
33 \\
19 \\
91 \\
26 \\
28 \\
15 \\
82 \\
8 \\
133 \\
127 \\
36 \\
74 \\
34 \\
105
\end{tabular} & \begin{tabular}{r|}
31 \\
470 \\
621 \\
43 \\
23 \\
8 \\
13 \\
43 \\
44 \\
21 \\
36 \\
86 \\
116 \\
75 \\
200 \\
24 \\
55 \\
29 \\
14 \\
17 \\
39 \\
130 \\
40 \\
14 \\
144 \\
36 \\
21 \\
24 \\
88 \\
8 \\
135 \\
140 \\
37 \\
75 \\
52 \\
158
\end{tabular} & $\begin{array}{r}12 \\
55 \\
810 \\
1125 \\
81 \\
39 \\
18 \\
20 \\
90 \\
84 \\
33 \\
65 \\
134 \\
196 \\
137 \\
359 \\
49 \\
76 \\
51 \\
29 \\
36 \\
83 \\
246 \\
78 \\
33 \\
235 \\
62 \\
49 \\
39 \\
170 \\
16 \\
268 \\
267 \\
73 \\
149 \\
86 \\
263\end{array}$ & $\begin{array}{l}0,33 \\
0,13 \\
0,14 \\
0,19 \\
0,15 \\
0,13 \\
0,11 \\
0,05 \\
0,21 \\
0,20 \\
0,06 \\
0,14 \\
0,06 \\
0,05 \\
0,17 \\
0,13 \\
0,24 \\
0,09 \\
0,14 \\
0,14 \\
0,17 \\
0,18 \\
0,13 \\
0,15 \\
0,21 \\
0,07 \\
0,13 \\
0,35 \\
0,03 \\
0,16 \\
0,25 \\
0,15 \\
0,18 \\
0,20 \\
0,24 \\
0,06 \\
0,08\end{array}$ \\
\hline Total & 52 & 60 & 156 & 277 & 559 & 592 & 473 & 495 & 156 & 128 & 859 & 1481 & 247 & 204 & 2562 & 3177 & 5739 & 0,15 \\
\hline
\end{tabular}

Source: Comité de Estado do Sector de Bolama, Recenseamento de 1987, Bolama, 1987, compilation interne. 
l'absence d'une telle force de travail sur la construction de digues dans les cas de rizières d'eau salée, sur les superficies labourées, sur toute l'agriculture en général." 1

\subsection{Ethnies}

Les analyses à caractère social de la Guinée-Bissau se doivent de considérer l'influence des nombreuses ethnies peuplant le pays. On y dénombre une quinzaine d'ethnies principales 2 dont cinq (Balante, Foula, Mandingue, Manjaque et Pepel) comptent pour plus de $80 \%$ de la population.

Ces ethnies peuvent être définies comme des collectivités caractérisées, et je cite:

..."par une même langue, une même tradition, culturelle et historique, par l'occupation d'un même territoire, par une même religion et surtout par la conscience collective d'appartenance à cette communauté..."3

Elles se situent "à mi-chemin entre la nation, dont "la dimension politique est plus marquée" et la civilisation "culturellement plus vaste""',4 et façonnent toutes, à leur manière propre, la réalité bissau-guinéenne.

Jean-Pierre Lepri distingue trois groupes sociaux principaux en Guinée-Bissau, et ce, à partir de leurs origines. 5 Le premier groupe, qu'il qualifie de celui des sociétés indigènes, est composé des ethnies originaires du pays. Il compte tant les sociétés ayant une organisation

1 Paul Rouillard, Réflexions sur le projet Bolama-Bijagos, op. cit. page 6.

2 Jean Brodeur, Guinée-Bissau, s.l., texte polycopié, septembre 1979, page 1.

3 Carlos Lopes, "Ethnie, Etat et rapports de pouvoir en Guinée-Bissau", Itinéraires, notes et travaux, No. 22, mars 1982, Première Edition, Genève, page 15.

4 Diana Lima Handem, Nature et fonctionnement du pouvoir chez les Balanta Brassa op.cit., page 47.

5 Jean-Pierre Lepri, "Contribução para a análise sociológica da Guiné-Bissau actual", Soranda:_Revista de Estudos Guineenses, No 1, Janeiro 1986, page 143. 
TABLEAU 2.6

Distribution ethnique

Région Bolama-Bijagos et ensemble du pays avril 1979

(en \%)

\begin{tabular}{|l|r|r|}
\hline \multicolumn{1}{|c|}{ Ethnie } & \multicolumn{1}{|c|}{$\begin{array}{c}\text { Bolama- } \\
\text { Bijagos }\end{array}$} & \multicolumn{1}{|c|}{$\begin{array}{c}\text { Guinée- } \\
\text { Bissau }\end{array}$} \\
\hline Balante & 3,76 & 27,20 \\
Beafada & 4,60 & 3,22 \\
Bijago & 61,13 & 2,52 \\
Foula & 2,41 & 22,92 \\
Mancagne & 9,34 & 3,33 \\
Mandingue & 3,39 & 12,21 \\
Manjaque & 2,46 & 10,62 \\
Pepel & 5,20 & 10,00 \\
Autres ethnies & 1,44 & 4,59 \\
Ethnie non-précisé & 6,27 & 3,30 \\
\hline \multicolumn{1}{|c|}{ Total } & 100 & 100 \\
\hline
\end{tabular}

Source: Ministério da Coordenação Económica e Plano, Recenseamento Geral da Populaçao e Habitacao, 16 de abril de 1979: resultados provisorios (fase II), Bissau, Departamento Central de Recenseamento, fevereiro 1982, page 133. 
relevant du mode de production communautaire (Balantes, Bijagos, Baiotes et Felupes), que celles associées au mode de production tributaire (Manjaques, Pepels, Brâmes). ${ }^{1}$

La pénétration de l'Tslam en Guinée-Bissau vers les $\mathrm{XII}^{\circ}$ et $\mathrm{XIV}^{\circ}$ siècles, déboucha sur l'existence de sociétés d'influence arabe. Ce second groupe, composé des ethnies Foulas, Beafadas et Mandingues, a comme cellule de base la famille mais qui, contrairement au groupe précédent, s'insère dans une structure hiérarchique pyramidale à caractère politicoreligieux, et est accompagné d'une structure de type professionnel. ${ }^{2}$

Le dernier groupe identifié est issu directement de cinq siècles de présence portugaise. Ce groupe, d'influence européenne, se retrouve presqu'essentiellement à Bissau et dans d'autres villes du pays et est composé des anciens "civilizados". Minorité dominante du pays, estimée à $5 \%$ de la population totale (contre $55 \%$ pour les sociétés indigènes et $40 \%$ pour celles d'influence arabe), Lepri n'hésite pas à l'identifier à une minorité dirigeante qui impose au pays ses valeurs typiquement européennes. 3

Bien que cette catégorisation de la société bissau-guinéenne nous soit d'une grande utilité dans la compréhension du pays en ce qu'elle nous permet de distinguer trois tendances culturelles au sein de cette formation sociale, nous opterons plutôt pour l'utilisation d'une autre grille privilégiant à une analyse historique (en terme d'origine), une analyse de la structure du pouvoir au sein de chaque ethnie, beaucoup plus proche des considérations propres aux modes de production. S'inspirant de la classification d'Amilcar Cabral, ${ }^{4}$ nous allons diviser la société bissau-guinéenne en sociétés verticales et horizontales, selon que le

1 Ibidem.

2 Ibid., pages 152-153.

3 Ibid., pages 144-158.

4 Amilcar Cabral, Unité et lutte, Paris, Petite Collection Maspero, 1980, pages 55-56. 
pouvoir y soit ou non centralisé, et la stratification sociale importante ou non. ${ }^{1}$ Les groupes ethniques qui seront régis par des structures sociales de type horizontal, sans structure de classes, bien qu'il puisse y avoir une certaine organisation du pouvoir, de droits ou de devoirs, selon l'état civil, l'âge ou le sexe, seront classés parmi les sociétés horizontales; ${ }^{2}$ à l'inverse, lorsqu'il y aura division de la société en classes, nous parlerons de sociěté verticale. Une relation peut être établie, dans la plupart des cas, entre mode de production communautaire et société horizontale et entre mode de production tributaire et société verticale. Nous aurons donc, d'un côté, les ethnies relevant du mode de production communautaire et que l'on pourra considérer comme des sociétés horizontales: les Balantes, les Baiotes, les Felupes et les Bijagos. ${ }^{3}$ De l'autre côté se retrouveront les sociétés verticales, relevant du mode de production tributaire, et se composant principalement des ethnies Foulas, Mandingues, Beafadas, Manjaques, Pepels et Brâmes (Mancagnes). ${ }^{4}$

Le Tableau 2.6 nous permet de constater que l'ethnie majoritaire de la région BolamaBijagos est l'ethnie bijago $(61,13 \%)$, suivie des ethnies mancagne $(9,34 \%)$, pepel $(5,2 \%)$, beafada $(4,6 \%)$ et mandingue $(3,39 \%)$. Comme nous l'avons maintes fois noté, l'ethnie bijago n'est pas la plus populeuse des ethnies habitant l'île de Bolama. Cependant, l'inexistence de données précises sur la situation ethnique de l'île de Bolama, nous aura forcé à porter une attention toute particulière sur la distribution ethnique de l'île lors de l'enquête de tabanca, puisqu'on remarque, dans les documents officiels nationaux, une tendance de plus

1 Carlos Lopes, "A Guiné-Bissau a procura de um modelo social", Soranda: Revista de Estudos Guineenses, No. 1, janeiro 1986, page 20.

2 Ministère de la Coordination Economique, du Plan et de la Coopération Internationale, La production populaire en Guinée-Bissau, Bissau, en collaboration avec OXFAM-Belgique, septembre 1985, Page 9.

3 Ibid. page 9;

Amilcar Cabral, op. cit., page 55; et,

Carlos Lopes, op.cit., page 21.

4 Ministère de la Coordination Economique, du Plan et de la Coopération Internationale, op.cit., page 9; Amilcar Cabral, op.cit., page 55; et, Jean-Pierre Lepri, op.cit., pages 152-153. 
en plus marquée à ignorer le fait ethnique et à ne considérer celui-ci que comme un vestige du passé. Cette forme de pensée est omniprésente aussi dans le discours des fonctionnaires et des membres du Parti, qui réduisent la réalité sociale du pays en une seule, la nationalité bissau-guinéenne.

L'enquête de tabanca aura donc dressé, pour les 25 villages consultés, la liste des ethnies principales et secondaires. Nous pouvons constater, au Tableau 2.7, la diversité ethnique de l'île de Bolama, diversité ne reposant pas sur quelques individus ou familles isolés, immergés dans un océan multi-ethnique, mais plutôt sur des villages à identité ethnique bien définie et qui conservent leurs modes de vie traditionnels. Des 25 villages enquêtés, 13 considèrent qu'ils sont peuplés en majorité de Mancagnes. Ils sont suivis des Balantes et des Bijagos avec trois villages chacun, des Pepels et des Foulas avec deux villages chacun, et enfin des Beafadas et des Mandingues avec un village chacun. Il importe de préciser que l'ethnie secondaire, lorsque présente dans un village, est souvent marginale en nombre et ne compte parfois qu'une ou quelques familles. A la lumière de ces quelques brèves considérations théoriques, nous nous proposons de préciser les principales caractéristiques de la plupart des ethnies présentes à Bolama, ethnies qui, en général, ne sont cependant pas originaires de l'île. Seule les Nhomincas, pêcheurs musulmans venus du Sénégal, ne seront pas traités vu leur faible importance démographique. Il n'en demeure pas moins que toutes les ethnies du pays et, par extension de Bolama aussi, présentent, au niveau de leurs modes de production, trois caractéristiques communes à toutes:

- la terre appartient à la communauté qui possède ses propres lois gérant sa distribution;

- la famille cultive la terre elle-même et n'a recours à autrui (familles ou individus) que pour les tâches requérant une main-d'oeuvre importante; et, 
TABLEAU 2.7

Ethnies principales et secondaires

selon le village

Ile de Bolama

1987

\begin{tabular}{|l|l|l|}
\hline \multicolumn{1}{|c|}{ Tabanca } & Ethnie principale & Ethnie secondaire \\
\hline Aranja Vida/Caboupa & Mancagne & Balante/Manjaque \\
\hline Balanbaï (Ponta Isidoro) & Balante & - \\
\hline Bairro Achamada & Bijago & Balante \\
\hline Bolama de Baixo & Bijago & Nhominca/Foula \\
\hline Calidje & Bijago & - \\
\hline Canema & Mancagne & Bijago \\
\hline Casseque & Mancagne & - \\
\hline Cassucaï & Mancagne & - \\
\hline Gâ Beafada & Beafada & Mandingue \\
\hline Gâ Moria & Mancagne & Bijago \\
\hline Gâ Nafa & Mancagne & Bijago/Foula \\
\hline N'Tacha & Pepel & Mancagne \\
\hline Lala & Mancagne & Balante \\
\hline Lala Balanta & Balante & Manjaque \\
\hline Luanda & Mancagne & Mandingue \\
\hline Madina & Foula & Manjaque/Bijago \\
\hline Marsasse & Mancagne & - \\
\hline Ponta Apili & Pepel & - \\
\hline Ponta Bissau & Mancagne & Balante \\
\hline Ponta Doce Balanta & Balante & \\
\hline Pontia & Mancagne & Balante \\
\hline Portugal & Mancagne & Manjaque \\
\hline Pujunguto & Mancagne & - \\
\hline Uato Fula & Foula & Bijago/Mancagne \\
\hline Uato Mandinga & Mandingue & Foula/Bijago/Mancagne \\
\hline & & \\
\hline & & \\
\hline
\end{tabular}

Source: D.P.R.-Bolama-Bijagos, Inquérito de tabanca, Bolama, avril 1987. 
- l'objectif fondamental de la production est l'autosuffisance alimentaire et, en cas d'excédent, celui-ci sera vendu ou troqué en vue de l'obtention de produits généralement non-agricoles. ${ }^{1}$

Ces trois aspects fondamentaux sont omniprésents dans chacune des ethnies, quel que soit son mode de production. On comprend que ces valeurs dictent toutes les conduites sociales ethniques et s'avèrent à la fois contrainte et atout dans le développement de ces communautés puisque:

"La connaissance des sociétés locales constitue un prérequis à toute opération de développement régional parce qu'elle permet d'identifier à la fois les valeurs propres au milieu régional et les éléments qui la rendent vulnérables au sousdéveloppement." 2

\subsection{Les sociétés horizontales}

Les deux exemples de société horizontale dont nous allons traiter correspondent adéquatement aux considérations théoriques que l'on peut trouver sur les sociétés régies par des systèmes de classes d'âge:

"Dans la catégorie générale des sociétés sans dirigeants, nombreuses sont celles dont le système politique et structurel élémentaire est fondé sur une hiérarchisation institutionnelle basée sur l'âge." 3

1 Carlos Cardoso, Carlos Rui Ribeiro, "Consideraçðes sobre as estruturas socio-económicas das sociedades agrárias e a sua evolução histórica; um estudo de caso", Soranda: Revista de Estudos Guineenses, Bissau, I.N.E.P., No. 3, janeiro 1987, page 9.

2 S.U.C.O.-C.R.A.D., Volume II, op. cit, page 40.

3 Adrian Colett, "L'autorité dans les communautés rurales africaines au Sud du Sahara", Le Courrier Afrique Caraibes Pacifique Communauté Européenne, No. 99, septembre-octobre 1986, page 69. 
A cette prémisse, l'auteur ajoute plus loin, après avoir constaté que ce type d'organisation sociale basé sur l'âge, répartit les individus en un certain nombre de classes d'âge, que:

"Dans ce genre de système politique, les décisions importantes sont prises par consensus, par les aînés et les anciens. L'autorité au jour le jour se manifeste par le respect qu'un homme d'un degré ou d'un échelon déterminé doit montrer à un autre d'un degré ou d'un échelon supérieur. Ce type d'autorité s'étend souvent en dehors des limites du village. Dans ce type de société, les femmes sont invariablement subordonnées aux hommes, l'épouse d'un aîné sera associée à l'échelon et au degré d'âge de son mari et elle aura à son tour autorité sur les épouses d'échelon inférieur. Les anciens sont souvent considérés comme ayant presque atteint le statut d'ancêtre. Ils conseillent plutôt qu'ils ne participent aux prises de décision de tous les jours. Ils peuvent aussi avoir des rôles rituels." 1

Le rôle des aînés dans les rites est une constante en Guinée-Bissau et de nombreux auteurs parlent de légitimation de l'autorité politique des vieux par la sacralisation. De ce fait, ce sont les fonctions religieuses qui permettent aux aînés de conserver et de jouir du pouvoir, celui-ci étant entouré de toute une gamme de rites, de cérémonies, d'initiations où l'individu acquiert le pouvoir et la connaissance tout au long de sa progression d'une classe d'âge à une autre, comme l'atteste le "fanadu", rite d'initiation balante:

"Le fanadu est en effet un lieu de sacralisation des autorités sociopolitiques qui est appréhendé comme le point de départ de la légitimation des charges publiques des aînés qui sera renforcée par le déploiement des fonctions religieuses qui leur seront attribuées au fil des années." 2

\subsubsection{Les Balantes}

La société balante représente l'un des extrêmes de la formation sociale bissauguinéenne, soit celui d'une société sans Etat, égalitaire, où chaque famille travaille selon ses

\footnotetext{
1 Ibid., page 69.

2 Diana Lima Handem, Nature et fonctionnement du pouvoir chez les Balanta Brassa op.cit., pages 145-146.
} 
propres besoins, sur une terre appartenant à la communauté, et où la femme participe au travail familial sur un pied d'égalité avec l'homme. ${ }^{1}$

Cette vision de la société balante reste cependant quelque peu idéaliste et la réalité est bien souvent beaucoup plus complexe que l'analyse qui ne verrait chez les Balantes, qu'une société horizontale dans laquelle "les catégories sociales seraient juxtaposées et entretiendraient des relations parfaitement égalitaires et antiautoritaires". ${ }^{2}$ En fait, chez les Balantes, on remarque une forme de stratification sociale basée sur le sexe et l'âge, où l'on passe d'une classe à l'autre et donc, d'un statut à l'autre, à mesure que l'on vieillit, chaque cadet devenant un jour aîné et pouvant ainsi jouir des privilèges relatifs à son âge. Malgré tout, une étude plus approfondie démontre que dans la société balante, des rapports d'inégalité apparaissent à partir de critères culturels faisant intervenir la parenté, le sexe et l'ancienneté de la famille en ce qui a trait à la possession de la terre. Les principales bases d'inégalité reposent sur la perception que la société se fera des individus, seuls ou en groupes, selon qu'ils soient hommes ou femmes, aînés ou cadets, maîtres de terre ou villageois, et qu'ils appartiennent ou non au lignage fondateur du village. ${ }^{3}$ Diana Lima Handem, dans son ouvrage sur les Balantes, affirme que les rapports d'inégalité prévalant chez les Balantes, dénotent un "phénomène social de différenciation institutionnalisée de trois ordres: sexuel, séniorial et segmentaire", qui varie selon qu'"il relève du domaine économique, politique, judiciaire ou législatif: 4

"Cette triple inégalité sociale traduit des relations de dépendance qui s'établissent en et hors parenté; et qui impliquent une organisation précise du travail ainsi que des pratiques sociales et politiques spécifiques. Les relations de

\footnotetext{
${ }^{1}$ Lars Rudebeck, Guinea-Bissau, A Study of Political Mobilization, Uppsala, The Scandinavian Institute of African Studies, 1974, page 10.

2 Diana Lima Handem, Lima, op. cit., page 65.

3 Ibid., pages 66-67.

4 Ibid., page 67.
} 
dépendance tissent un réseau de communication qui prend forme d'obligation et de privilège, opposant l'homme à la femme, le blufo à l'alante ndã [jeune homme non initié à l'homme initié de plus de 45 ans] et le lignage postérieur au lignage fondateur. La prépondérance de l'homme et du patrilignage dans les affaires publiques, ainsi que le prestige et les privilèges sociaux attachés à la séniorité et déterminants des fonctions sociales de balante-badā [initiés mâles] et du fan bodja - le mâ̂tre de terre, dénommé dó di tcho en créole - sont autant de prérogatives qui définissent la stratégie du réseau de dépendance propre à la société arassa et révèlent son mode de traitement de l'inégalité. Cette stratégie, qui présente diverses figures pratiques, met en jeu des relations sociales instituées légalement par des règles de prestation et de préséance, et moralement par des normes valorisantes. Elles sont par ailleurs renforcées par des signes, des symboles et des prohibitions. Le réseau des relations de dépendance confirme ainsi le statut des individus et des groupes sociaux, au sein de la famille et du lignage, dans la morasa [ [habitation] comme dans la tabanca [village].

L'inégalité qui sous-tend les relations de dépendance trouve sa justification dans deux registres idéologiques qui permettent de survaloriser, d'une part l'appartenance au sexe masculin "fort", et d'autre part, la qualité de premier par la naissance et par l'occupation territoriale.

La survalorisation au niveau idéologique de ces deux états de fait, constitue le substrat de la distribution des privilèges et du partage des charges entre les partenaires sociaux classés dans les différentes hiérarchies." 1

Malgré ces quelques réserves à l'endroit de l'égalitarisme des Balantes, il n'en demeure pas moins que ceux-ci n'ont traditionnellement ni Etat, ni chef, de telle sorte que, pour mieux les contrôler, les colonisateurs portugais leur imposèrent des chefs d'autres ethnies, notamment de l'ethnie mandingue. ${ }^{2}$ On remarquera surtout, chez les Balantes, outre leur absence de chef et leur recours au conseil des anciens pour régler toute question problématique, leur désir d'indépendance qui se traduit par le fait que chaque famille et chaque habitation est autonome, ${ }^{3}$ comme le souligne Cabral:

"Il arrive même dans ce groupe que deux habitations se trouvent côte à côte, sans que jamais il ne soit établi un quelconque échange entre elles, soit pour une affaire de terres, ou pour tout autre problème survenu dans le passé. Ils ne veulent rien attendre les uns des autres." 4

\footnotetext{
1 Ibid., pages 67-68.

2 Amilcar Cabral, Unité et lutte, op. cite, page 55.

3 Jean-Pierre Lepri, "Contribução para a análise sociológica da Guiné-Bissau actual", op. cit., page 146.

4 Amilcar Cabral, op. cit, page 55.
} 
A ce désir d'indépendance des Balantes qui d'ailleurs, notons-le, possèdent une technique de production de riz en eau salée très développée, s'ajoute le fait qu'ils n'encouragent pas la richesse individuelle, toute richesse étant faite pour être dépensée ou consommée et non accumulée, comme en fait preuve la coutume voulant qu'à la mort d'un Balante, la communauté consomme en totalité les biens du défunt (animaux, nourriture, etc.).

\subsubsection{Les Bijagos}

Comme le premier chapitre le précisait, la société bijago repose sur trois éléments clés: la famille, la classe d'âge et la religion.

Chez les Bijagos, la répartition des terres s'effectue en référence à l'appartenance à un clan. Traditionnellement, toutes les terres bijagos appartiennent à l'un ou l'autre des quatre clans matrilinéaires existants et l'individu se voit attribuer des terres en fonction du clan d'appartenance de sa mère. Au contraire, la résidence se transmet de façon patriarcale: ainsi, bien qu'un jeune homme appartienne au clan de sa mère, il habitera près de chez son père qui lui, est d'un clan différent. 1

Sur cet aspect, la société bijago correspond parfaitement au modèle de passage d'une société nomade à une société d'agriculteurs, où la femme, procréatrice, assure la postérité nécessaire à l'appropriation collective de la terre: "la maternité devient une fonction sacrée".2 Le clan s'identifie à la permanence du sol, de la terre, et ainsi prend conscience de l'importance du passé et de l'avenir, respecte et vénère ses ancêtres totémiques, reconnaît l'importance de sa descendance. 3 Apparait alors un lien profond entre la femme, la terre,

1 S.U.C.O.-C.R.A.D., Volume II, op.cit., pages 40-41.

2 Simone de Beauvoir, Le deuxième sexe, s.l., Editions Gallimard, 1949, Tome 1, page 85.

3 Ibid., pages 85-86. 
toutes deux génératrices, et le clan, qui ne se contente plus de la simple cueillette des fruits de la terre, et développe un sentiment ambiguë face aux forces de la nature, alliant magie et technique. ${ }^{1}$ D'une manière théorique donc, et ceci s'applique aussi à la société bijago:

"Très souvent les enfants appartiennent au clan de leur mère, portent son nom, participent à ses droits et particulièrement à la jouissance de la terre que le clan détient. La propriété communautaire se transmet alors par les femmes: par elles les champs et les moissons sont assurés aux membres du clan et inversement c'est par leurs mères que ceux-ci sont destinés à tel ou tel domaine. On peut donc considérer que mystiquement la terre appartient aux femmes: elles ont une emprise à la fois religieuse et légale sur la glèbe et ses fruits. Le lien qui les unit est plus étroit encore qu'une appartenance; le régime de droit maternel se caractérise par une véritable assimilation de la femme à la terre; en toutes deux s'accomplit à travers ses avatars, la permanence de la vie, la vie qui est essentiellement génération." 2

Tout comme pour les Balantes, on note chez les Bijagos l'existence de classes d'âge que l'on franchit par des rites d'initiation:

"C'est à travers les rites de l'initiation ("manratche") que les Bijagos passent d'une classe d'âge à l'autre, changement qui implique une augmentation de leurs privilèges et une diminution de leurs obligations, à mesure qu'ils avancent en âge. D'après Santos Lima (1947), l'initiation comprend chez les Bijagos trois phases principales, les deux premières pouvant être considérées comme un "noviciat" et la troisième comme l'étape qui permet d'acquérir le statut et les droits de l'adulte." 3

L'homme atteindra la seconde classe d'âge à partir d'une vingtaine d'années et ne sera considéré adulte qu'à la trentaine avancée. L'initiation chez la femme est restée assez secrète et ses modalités nous sont encore inconnues. 4

\footnotetext{
1 bid., page 88.

2 Ibid., page 87.

3 S.U.C.O.-C.R.A.D., Volume II, op. cit., page 44.

4 Ibid., pages 44-46.
} 
Tout comme la société balante, la société bijago est une gérontocratie gouvernée par un conseil des anciens au niveau des villages, qui gère les conflits et détient le pouvoir législatif et juridique. ${ }^{1}$

Les Bijagos vivent dans un système d'economie de subsistance où l'on assure, à tous les membres de la société, la subsistance par l'allocation universelle des ressources et le principe d'entraide envers toute personne dans le besoin. Parallèlement à cela, les modalités de production et de distribution des biens de subsistance reposent sur les obligations familiales, religieuses et morales de chacun. ${ }^{2}$ Il n'est donc pas étonnant de constater que la plupart des activités ont un caractère communautaires et que la prise des décisions économiques est fortement décentralisée. Il en découle que le processus de prise de décision d'ordre économique est forcément lent, de par les nombreuses négociations qu'il sous-tend, et que son caractère communautaire a pour effet de décourager et même de rejeter toute innovation individualiste qui pourrait avoir un effet sur plusieurs membres de la communauté. ${ }^{3}$ De plus, il existe une attitude de rejet de la part de la communauté face à toute initiative visant l'enrichissement matériel (monétaire). Ceci, en plus de décourager toute tentative individuelle en la matière, marginalise à un point tel les individus qui sont effectivement animés d'un désir d'enrichissement monétaire ou d'accumulation matérielle, qu'ils seront peu à peu exclus du village et iront ainsi souvent construire leur case à l'extérieur du village.

\footnotetext{
1 Ibid., page 46.

2 Ibid, page 64.

3 Ibid., page 53.
} 
Animistes comme les Balantes et en majorité monogames, ${ }^{1}$ quoique les femmes aient une certaine liberté dans le choix et la succession de leurs époux ou amants, ${ }^{2}$ les Bijagos cultivent le riz pluvial, base de leur alimentation, qu'ils additionnent d'huile de palme et de mollusques ou de poisson à l'occasion. Le régime alimentaire bijago se trouvera complété de quelques légumes et céréales cultivés (mil, haricot, patate douce, manioc, courge et pois de terre) et de fruits (mangues, cajous, oranges et papayes). ${ }^{3}$

\subsection{Les sociétés verticales}

L'étude des sociétés verticales sera abordée en deux étapes, une première traitant de trois ethnies animistes (Manjaques, Pepels et Brâmes/Mancagnes) et la seconde de trois ethnies islamisées (Foulas, Mandingues et Beafadas).

\subsubsection{Les Manjaques, Pepels et Brâmes/Mancagnes}

Nous avons regroupé ces trois ethnies parce qu'elles présentent des traits communs au niveau des habitudes de vie, des traditions, des techniques de travail et de la langue. ${ }^{4}$ Bien qu'animistes, elles se situent en quelque sorte entre les sociétés sans classe de type balante et les sociétés de classes, fortement hiérarchisées du genre de celle des Foulas. ${ }^{5}$ Cet état de fait

\footnotetext{
1 Danielle Gallois Duquette, Dynamique de l'art bidjoge (Guinée-Bissau): contribution à une anthropologie de l'art des sociétés africaines, Lisbonne, Instituto de Investigação Científica Tropical, 1983, page 20.

2 Christine Henry, Hommes seuls et femmes volages, note sur le mariage des Bijagos de Caravela, texte nonpublié, s.l., juin 1984, 28 pages.

3 S.U.C.O.-C.R.A.D., Volume II, op. cit., page 49.

4 António Carreira, A etnonímia dos povos de entre o Gâmbia e o estuário do Geba, Bissau, 1964, pages 252 253.

5 Carlos Lopes, A transicăo histórica na Guiné-Bissau: de movimento de libertacão nacional ao Estado, Genève, Mémoire présenté pour le Diplôme de Recherche en Etudes du Développement, Institut Universitaire d'Etudes du Développement, novembre 1982, page 18.
} 
explique sans doute l'ambivalence qui prévaut dans l'étude de ces sociétés, les uns les classant en partie parmi les sociétés horizontales, ${ }^{1}$ les autres les situant dans les sociétés verticales. ${ }^{2}$ La classification de ce groupe d'ethnies se révèle assez difficile et d'une ambiguitté Évidente. En effet, ces ethnies présentent des caractéristiques culturelles semblables à celles des sociétés horizontales, comme l'indique une publication du Ministère du Plan bissauguinéen en parlant de ce groupe et des sociétés horizontales:

..."les classes d'âge et les initiations (les "fanado") jouent un grand rôle dans la répartition des tâches, l'organisation du travail et la prise des décisions sociales. Leurs économies sont peu monétarisées, l'épargne se faisant souvent en bétail ou en stock de riz, et la capitalisation est faible, les richesses d'un individu étant consommées à sa mort par la communauté.

Pour cette raison, l'introduction des nouveautés, par le biais de l'initiative d'une personne seule, est souvent condamnée à l'échec. Néanmoins ces sociétés ne sont pas - comme on les présente parfois - ni conservatrices ni destinées à l'immobilisme; il y a même des changements importants qui ont été constatés, depuis la guerre de libération, au niveau de l'organisation sociale et l'accès des jeunes au pouvoir.

Pour la vulgarisation rurale et l'introduction de nouvelles technologies, il est nécessaire d'attendre, toutefois, des décisions prises en communauté, avant qu'une initiative ait une chance de réussir." 3

Ce groupe d'ethnies partage aussi une aire d'habitation commune avec les ethnies des sociétés horizontales et l'histoire nous enseigne d'ailleurs que lors de la pénétration de l'Tslam en Guinée-Bissau, à partir du XIII ${ }^{\circ}$ siècle, toutes ces ethnies ont été repoussées par l'envahisseur Foula et Mandingue vers la côte, ${ }^{4}$ si bien que de nos jours, les ethnies animistes se concentrent sur tout le littoral du pays jusqu'à la limite d'influence des marées,

\footnotetext{
1 Ibid, pages 25-29.

2 Jean-Pierre Lepri, op.cit., page 146; et, Ministère de la Coordination Economique, du Plan et de la Coopération Internationale, La production populaire en Guinée-Bissau, op.cit., page 9.

3 Ibid., page 9.

4 Lars Rudebeck, Guinea-Bissau, A Study of Political Mobilization, op. cit., page 11.
} 
alors que les peuples islamisés habitent surtout à l'intérieur des terres ${ }^{1}$ et ce, à l'exception seule des Beafadas qui vivent près de la côte, notamment dans le secteur de São-João.

Il convient d'ajouter à ces similitudes entre ce groupe et les deux ethnies relevant des sociétés horizontales que nous avons précédemment étudiées, un désir commun de résistance à l'envahisseur colonial portugais. Ainsi, on note que les Pepels ont résisté à la pacification portugaise jusqu'en 1936, et ce, aux portes mêmes de Bissau. ${ }^{2}$

La différence entre les sociétés horizontales étudiées et ces trois ethnies, apparaît lorsque l'on se penche sur leur modèle d'organisation sociale, très hiérarchisé, où règnent rois, chefs et notables. ${ }^{3}$ Le régime foncier des Pepels, par exemple, est assez complexe. La terre peut appartenir à certaines classes et de nombreux villages se retrouvent devant l'obligation de louer les terres qu'ils cultivent. ${ }^{4}$ Leur système de distribution des terres est lié aux cérémonies de mariage et aux classes d'âge, et la structure familiale est elle-même des plus variées et des plus compliquées. ${ }^{5}$ Le peuple pepel cultive, pêche et, comme les Manjaques, est un grand cueilleur du fruits de palmier à partir desquels il tire une huile, l'huile de palme. Le tissage est très développé chez cette ethnie où l'on retrouve dans chaque village un forgeron et toute une gamme d'artisans. ${ }^{6}$ La spécialisation du Pepel dans la cueillette des régimes de palme en fait un incessant nomade, allant d'une région du pays à l'autre, selon la disponibilité des palmiers. A la différence des Manjaques, les Pepels, lors de leurs migrations saisonnières, ne laissent pas leurs villages inhabités; ils y reviennent pour la culture du riz en saison des pluies. Les mouvements migratoires pepels les amènent en assez

1 Carlos Cardoso, Carlos Rui Ribeiro, "Considerações sobre as estruturas socio-económicas das sociedades agrárias e a sua evolução histórica; um estudo de caso", op. cit., page 11.

2 Lars Rudebeck, op. cit, page 11.

3 Ibidem.

4 Jean-Pierre Lepri, op cit., page 151.

5 Carlos Lopes, op cit. pages 28-29.

6 Ibid., page 29. 
grand nombre dans les îles de l'archipel bijago (hormis Bolama) qui sont très riches en palmiers. Hochet et Uldely notent cependant que depuis quelques années, les Pepels ont tendance à se défricher un champ pour la riziculture lors de leurs séjours dans l'archipel.1 Cette tendance peut présager une certaine sédentarisation si elle se poursuit. A ce titre, certains villages pepels de l'archipel semblent vouloir servir de plus en plus de villages permanents, comme c'est le cas à Ankadidja sur lî̀le de Bubaque, où l'on s'adonne au maraîchage et à la culture du riz pluvial. 2

Les Brâmes sont également connus sous le nom de Mancagnes et représentent un des 13 clans de cette ethnie. Ces clans possédaient une base totémique mais sont, de nos jours, très difficiles à distinguer les uns des autres. ${ }^{3} \mathrm{Il}$ importe de préciser que, tout au long du mémoire, nous allons privilégier l'utilisation du terme mancagne pour définir cette ethnie ainsi désignée à Bolama. Les Mancagnes pratiquent une culture itinérante et diversifiée. Leurs liens avec la terre sont importants et son allocation s'effectue sur une base familiale par les anciens du village. De par leur habitude de la culture itinérante, les Mancagnes se considèrent nomades, comme tend à la prouver leur interprétation de leur installation à Bolama qu'ils ne considèrent que passagère, ${ }^{4}$ bien qu'ils constituent l'ethnie la plus populeuse de l'île.

\subsubsection{Foulas, Mandingues et Beafadas}

L'ethnie mandingue, grâce à ses impressionnantes conquêtes lors du puissant empire du Mali au XIV ${ }^{\circ}$ siècle, s'est forgée une forte réputation en Afrique de l'Ouest. Les premiers

\footnotetext{
1 Anne-Marie Hochet, Seco Uldely, Les ex-royaumes Pepel du Tor et du Biombo: zones d'émigration temporaire: situation socio-économique, Guinée-Bissau, Commissariat à la Coordination Economique et au Plan, novembre 1980, Pages 4-6.

2 Brigitte Boies, Alain Rousseau, Guide pour l'analyse des systèmes de production: archipel Bolama-Bijagos. Guinée-Bissau, Bolama, Projet de Développement Agricole Bolama-Bijagos, C.E.C.I., mai 1988, page 22.

3 António Carreira, op.cit. page 263.

4 Carlos Lopes, op. cit., page 25.
} 
Mandingues à arriver en Guinée-Bissau furent des guerriers, dès le $\mathrm{XIII}^{\circ}$ siècle. Ils repoussèrent peu à peu les autres ethnies vers la côte et se convertirent à l'agriculture. 1

Les Foulas, descendants de la famille peul, ${ }^{2}$ sont arrivés en Guinée-Bissau à peu près en même temps que les Mandingues mais leur influence s'est fait surtout sentir aux XVIII ${ }^{\circ}$ et $\mathrm{XIX}^{\circ}$ siècles. De pasteurs qu'ils étaient à l'origine, les épidémies de trypanosomiase affectant leur bétail les ont progressivement forcés à devenir sédentaires et à adopter des pratiques agricoles semblables à celles des Mandingues. 3

Il semble que les Foulas et les Mandingues de la Guinée-Bissau actuelle, ne soient pas tous descendants directs des Foulas et Mandingues qui envahirent le pays, mais plutôt des membres d'autres ethnies, originaires de Guinée-Bissau, et qui furent assimilés par les conquérants. Ceci explique sans doute les différences notables que l'on constate entre les Foulas et les Mandingues des pays voisins et ceux de la Guinée-Bissau. D'ailleurs, on raconte que les Balantes ont refusé systématiquement de se soumettre à l'envahisseur d'où, paraît-il, viendrait la signification du nom de leur ethnie voulant dire "ceux qui résistent".4

La société foula est diamétralement opposée à celle des Balantes au niveau de sa structure sociale. Elle se caractérise par une très forte hiérarchisation où les chefs exploitent leurs paysans. La société mandingue partage une organisation sociale similaire quoique moins rigide et moins hiérarchique. 5

1 Simone Payeur,La riziculture de bas-fonds en Guinée-Bissau: contraintes et possibilités de développement dans le cadre du projet de développement rural de la Zone II. Montpellier, Centre National d'Etudes Agronomiques pour les Régions Chaudes, Ecole Supérieure d'Agronomie Tropicale, janvier 1982, page 29.

2 Jean Brodeur, Guinée-Bissau, op.cit., page 1.

3 Simone Payeur, op.cit, page 29.

4 Amilcar Cabral, Unité et lutte, op. cit., page 56.

5 Lars Rudebeck, op. cit, pages 10-11. 
Les ethnies foula, beafada et mandingue ont comme cellule de base la famille, famille qui s'insère, contrairement aux autres ethnies du pays, dans une structure pyramidale de type politico-religieuse accompagnée d'une structure de type professionnelle. ${ }^{1}$ Leurs sociétés, très hiérarchisées, sont islamisées, polygames et on y connaît la propriété privée. ${ }^{2}$ Simone Payeur dresse ainsi le portrait des ethnies foula et mandingue:

"L'organisation familiale est de type patriarcal chez les deux ethnies. Au chef de famille et à son ménage, s'ajoutent ses frères cadets (avec leurs femmes et enfants mariés et célibataires), parfois des enfants confiés à la famille. La polygamie est largement répandue. La grande famille, traditionnellement unité de base de la société, a plus ou moins éclaté sous l'effet de la guerre, de l'émancipation des cadets et des jeunes, accentuée par les revenus monétaires; elle semble néanmoins presque toujours caractérisée par l'unité de résidence. Néanmoins, au sein d'une grande famille, on pourra trouver des unités de production autonomes (UPA) qui cultivent en commun leurs champs de céréales, en stockent le contenu dans des greniers communs, possèdent leur propre bétail et disposent de certains revenus monétaires, une partie pouvant être conservée par le ménage, voire même l'individu" ... ${ }^{3}$

De la polygamie pratiquée chez ces ethnies découle que, chez les Foulas par exemple, les femmes n'ont pas de droit et, même si elles participent à la production, elles n'ont aucun droit sur le produit, en fait, les hommes considèrent les femmes comme leur propriété. 4

A cette distinction homme/femme s'ajoute une structuration hiérarchique de la société où, au sommet, dominent les chefs de village, suivis des chefs religieux. Ces deux groupes forment la classe dirigeante. Sous leur autorité on compte tous les membres des diverses

\footnotetext{
1 Jean-Pierre Lepri, "Contribução para a análise sociológica da Guiné-Bissau actual", op cit., pages 152-153.

2 Danielle Gallois Duquette, Dynamique de l'art bidjogo (Guinée-Bissau): contribution à une anthropologie de la'art des sociétés africaines, op. cit., page 20.

3 Simone Payeur, op. cit. pages 29-30.

4 Lars Rudebeck, op. cit, page 10.
} 
professions (cordonnier, forgeron, orfevre et autres artisans) et enfin la grande masse, celle des paysans qui cultivent la terre pour leurs chefs, selon la tradition. ${ }^{1}$

On peut donc comprendre, d'une manière générale, qu'il semble y avoir, en GuinéeBissau, une relation entre les structures sociales ethniques et les religions adoptées par ces mêmes ethnies. L'islam domine chez les sociétés les plus hiérarchisées alors que les sociétés horizontales sont de croyance animiste. La relation entre religion et structure sociale serait parfaite si ce n'est du groupe des Manjaques, Pepels et Mancagnes qui, bien que disposant de sociétés relativement hiérarchisées, ne sont que très peu imprégnés des croyances musulmanes. ${ }^{2}$ Quoiqu'il en soit, il peut être intéressant de soulever le fait qu'en GuinéeBissau, toutes les ethnies ont su conserver un fond de croyances traditionnelles:

"Les populations musulmanes sont au fond aussi animistes que les Balantes ou d'autres. Elles croient en Allah, mais aussi à l'Iran [esprit] et aux djambacosses [sorciers guérisseurs]. Elles ont le Coran mais elles portent des grigris." 3

Les différences inter-ethniques sont malgré tout nombreuses, tant au niveau des spécialisations de chacune qu'à celui de leurs structures sociales. La religion, les structures sociales et la culture de chaque ethnie auront façonné un pays diversifié où les différences ethniques ont su être exploitées par le colonisateur qui n'a pas craint de s'allier les ethnies fortement hiérarchisées (Foulas et Mandingues) et plus ouvertes à l'accumulation de richesses soit par l'enrichissement individuel, soit par héritage (Foulas, Mandingues et Beafadas), ${ }^{4}$ et

1 Amilcar Cabral, op.cit., pages 56-57.

2 Lars Rudebeck, op. cit, page 11.

3 Amilcar Cabral, op cit. page 79.

4 Ministère de la Coordination Economique, du Plan et de la Coopération Internationale, La production populaire en Guinée-Bissal, op cit., page 9. 
ainsi utiliser leur structure sociale traditionnelle afin d'impliquer chefs, notables et rois dans la réalisation de leurs propres intérêts colonialistes. ${ }^{1}$

Dans le prochain chapitre, nous dresserons le profil socio-économique de l'île de Bolama, et ce sera l'occasion, entre autres, de nous familiariser avec les pratiques propres à chaque ethnie sur le plan de la production.

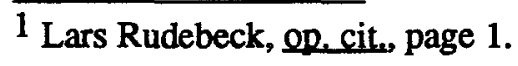




\section{CHAPITRE TROIS}

\section{LE MILIEU SOCIO-ECONOMIQUE BOLAMIEN}

Bien que l'on constate la présence d'un centre urbain sur l'île de Bolama, celle-ci a tout de même conservé le caractère rural propre au pays. Ainsi, $54,2 \%$ de la population bolamienne, ${ }^{1}$ de même qu'une proportion non négligeable de citadins s'adonnent à l'agriculture, une agriculture de subsistance, peu variée si ce n'est des spécialisations de chaque ethnie: riz d'eau salée (chez les Balantes), riz d'eau douce (Bijagos), riz pluvial (la majorité des ethnies), arachides (Mancagnes), maraîchage (Mancagnes) ou encore élevage (Foulas). ${ }^{2}$ Les activités reliées à l'administration, à l'éducation, à la santé, au commerce ou la production manufacturière se regroupent à Bolama et n'occupent et ne s'adressent qu'à une minorité. En effet, quiconque observe la ville de Bolama, est immédiatement frappé par le désoeuvrement apparent qui touche la population bolamienne et, tout particulièrement, les jeunes. Malgré qu'aucune donnée précise concernant le taux de chômage de la population n'existe, il n'en demeure pas moins que nous pouvons, par observation, affirmer que le chômage touche la très grande majorité des jeunes de moins de 25 ans et des femmes. Un rapide calcul faisant intervenir la source majeure d'emploi, la fonction publique (715

1 Ce chiffre est obtenu à partir des renseignements contenus dans le Tableau 2.4 selon lesquels, 3042 personnes sur 5616 habitaient en campagne en 1987.

2 Brigitte Boies, Alain Rousseau, Guide pour l'analyse des systèmes de production; archipel Bolama-Bijagos. Guinée-Bissau, Bolama, Projet de Développement Agricole Bolama-Bijagos, C.E.C.I., mai 1988, 26 pages + Annexes. 
personnes), qui inclut tant les employés de l'administration publique que les personnes travaillant dans les diverses entreprises étatiques, que ce soit les commerces d'Etat, le Port, l'Imprimerie Nationale, Titina Sila, le comité dEtat, etc., porte le taux des sans travail chez la population urbaine totale des 16 a 60 ans à $34,2 \%$. Une estimation, qui nous semble généreuse fait descendre ce taux sous les $30 \%$, si l'on tient compte des personnes travaillant dans le commerce privé (magasins et marché), les projets de coopération, la pêche et le travail de domestique, de même que de celles aux études mais domiciliées à Bolama qui, en fait, ne comptent que pour une infime partie des étudiants de ce niveau et de cette classe d'âge, la majorité étant plutôt composée d'individus venant d'un peu partout dans le pays. Cependant, dans la réalité, le taux réel des sans emploi doit dépasser nettement les $30 \%$ puisque la population urbaine nous semble plus importante en nombre que ce que les chiffres du recensement du comité de secteur pourraient nous faire croire. De plus, nous devons prendre en considération que le travail au champ est saisonnier (la saison des pluies), et la division sexuelle du travail laisse l'homme plus libre que la femme une bonne partie de l'année. Cette liberté permet un certain mouvement migratoire vers la ville, de personnes à la recherche d'un emploi, et qui peuvent profiter de l'étendue et de l'hospitalité de la famille africaine. Nous avons maintes fois pu observer ce phénomène, et l'on n'a qu'à se promener en ville et dans les quartiers périphériques de Bolama pour constater que la population sans travail y est apparemment nombreuse.

Ce chapitre vise avant tout à nous faire connaître le contexte socio-économique de l'île de Bolama. Nous dresserons ainsi un bref portrait des différents champs d'activité de l'île, de même que des principales conduites, individuelles ou ethniques, de la population bolamienne en regard du travail et du domaine socio-économique en général. Il convient avant tout de prendre connaissance du calendrier annuel typique du déroulement des principales activités (agriculture, pêche, cueillette) qui se pratiquent à Bolama. Les différents ouvrages faisant état 
jusqu'ici d'un calendrier des activités, ${ }^{1}$ l'ont fait pour l'ensemble de la région BolamaBijagos et, celle-ci étant peuplée en majorité de Bijagos, les modèles de calendriers proposés sont donc basés sur les activités propres à cette ethnie. La conséquence la plus évidente est, qu'à Bolama, contrairement aux autres îles, la récolte des régimes de palmistes et la production d'huile de palme, ne constituent pas des activités très courantes étant donnée la rareté des palmiers. Une étude des superficies totales couvertes par les palmiers sur les différentes îles de l'archipel, démontre en effet qu'à Bolama, la couverture de palmiers n'atteint que 3,3\% de la superficie totale de l'île alors qu'ailleurs, elle dépasse dans plusieurs cas les 50\%: João Vieira et Rubane, îles qui ne sont pas habitées à l'année, $69,4 \%$ et 54,3\%, Soga, $60,4 \%$, Caravela, $62 \%$, Cute, $60 \%$, etc.. ${ }^{2}$ Hormis cette pratique et, par extension, celle de la cueillette des coeurs de palmiers, nos observations nous ont permis de constater que le modèle de calendrier que nous reproduisons au Tableau 3.1, correspond en gros, à la réalité bolamienne.

\subsection{Agriculture}

Le mode traditionnel d'attribution des terres est encore en vigueur à Bolama bien qu'après l'indépendance, l'Etat s'appropria toutes les terres qui étaient libres. ${ }^{3}$

\footnotetext{
1 S.U.C.O.-C.R.A.D., Le développement rural intégré de la région de Bolama. République de Guinée-Bissau, Université Laval, Maîtrise en Aménagement du Territoire et Développement Régional, 1982, Volume I, page 50; Marthe Côté, André Demers, Repères pour un développement rural intégré dans la région de Bolama. GuinéeBissau, Université Laval, Maitrise en Aménagement du Territoire et en Développement Régional, novembre 1982, pages 94-95; et,

Jacques Bitard, Appui à l'aqriculture dans les îles Bijagos et développement des moyens de production agricole, s.l., F.E.N.U., GBS/85/CO3, avril 1987, page 198.

2 S.U.C.O.-C.R.A.D., Le développement rural intégré de la région de Bolama. République de Guinée-Bissau, Université Laval, Maîtrise en Aménagement du Territoire et Développement Régional, 1982, Volume II, page 185.

3 Ibid., page 121.
} 


\section{TABLEAU 3.1}

Calendrier des principales activités

Région Bolama-Bijagos (1)

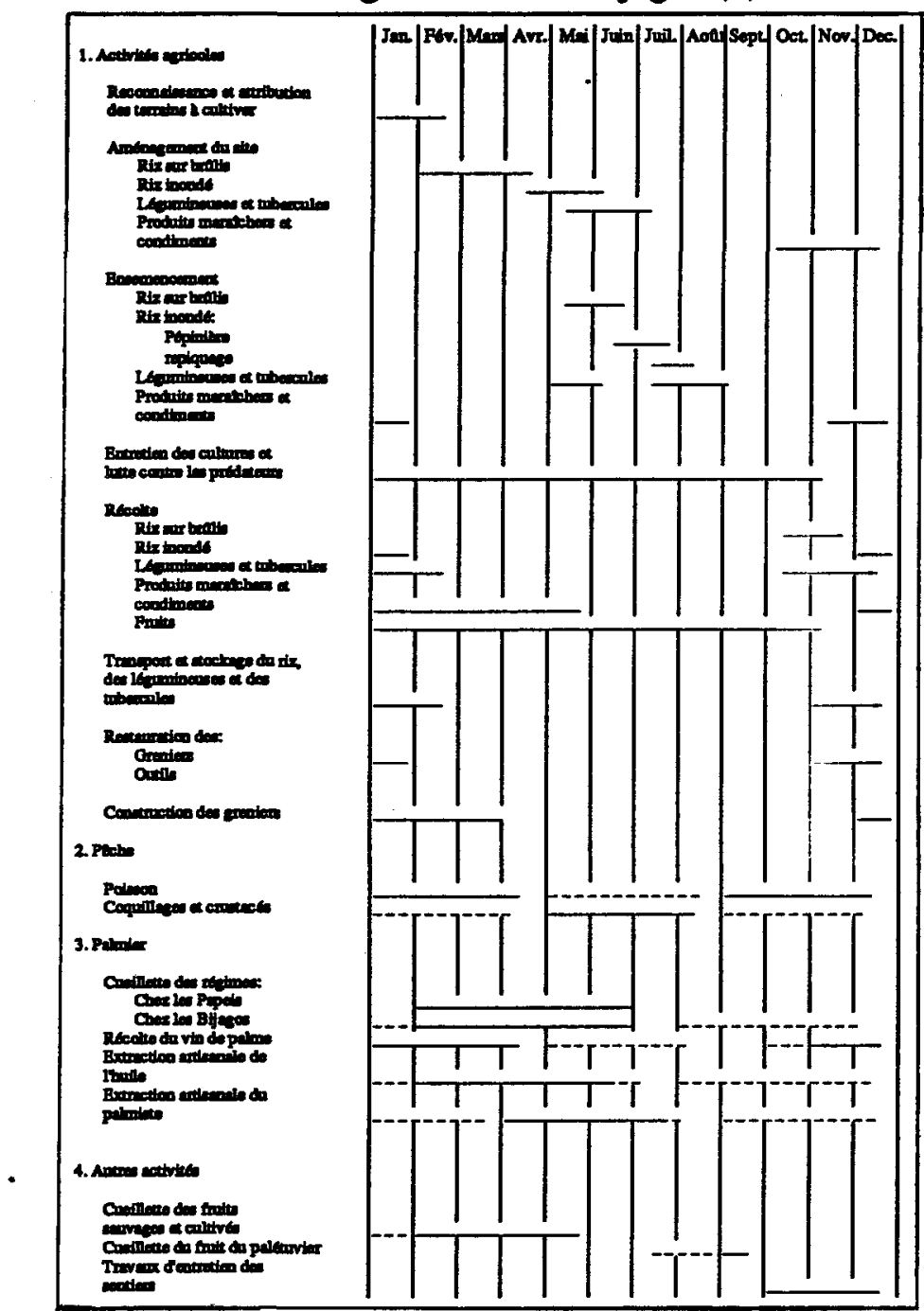

(1): Les traits continus ( $\_$) indiquent les mois où se déroule l'activité; les traits pointillés ( _ _ - ) sont utilisés lorsque l'activité est intermittente.

Source: S.U.C.O.-C.R.A.D., Le développement rural intégré de la région de Bolama, République de Guinée-Bissau, Volume I, Université Laval, Maîtrise en Aménagement du Territoire et Développement Régional, 1982, page 50; et COTE, Marthe, DEMERS, André, Repères pour un développement rural intégré dans la région de Bolama, Guinée-Bissau, Université Laval, Maîtrise en Aménagement du Territoire et en Développement Régional, novembre 1982, pages 94-95. 
A Bolama, chaque village contrôle un certain territoire sous la responsabilité du chef qui procède à une répartition égalitaire de celui-ci parmi les chefs de famille du village, selon la force de travail dont ils peuvent disposer et leurs besoins propres. Chaque paysan a le droit d'usage permanent de la terre qui lui a été attribuée et peut en faire bénéficier ses descendants après sa mort. Le chef du village demeure le responsable de la répartition du terroir et, dans le cas de litiges au sujet des terres, comme cela peut arriver pour l'administration d'une rizière par exemple, il lui revient le droit et le devoir de trancher. C'est aussi le chef, qui attribue les terres dans le cas d'étrangers convoitant une partie des terres villageoises. ${ }^{1}$

Nous avions, lors d'un précédent chapitre, fait état du processus bijago d'attribution des terres, basé sur l'appartenance clanique. Cette méthode n'est pas pratiquée sur l'île de Bolama, celle-ci se trouvant hors des zones des clans, limitées au véritable archipel bijago. A Bolama, les Bijagos effectuent donc de manière semblable aux autres ethnies, l'allocation des terres, en prenant soin de procéder au rituel adressé aux esprits qui, chez toutes les ethnies, possèdent le fond de terre, le paysan n'en réclamant que le droit d'usage. ${ }^{2}$

Ce mode traditionnel d'attribution des terres semble donner satisfaction:

"Depuis l'indépendance, tous les fonds de terre qui n'étaient pas déjà des propriétés privées dûment reconnues sont devenues propriétés de l'Etat. Mais comme le faisait remarquer une autorité politique locale, à propos d'une Éventuelle intervention du gouvernement dans la réfection et l'agrandissement de la rizière en eau salée de Lala à Bolama, les coutumes traditionnelles d'attribution des terres sont très efficaces et elles éliminent généralement les injustices par une répartition égalitaire aux paysans. C'est pourquoi l'Etat n'a pas à intervenir directement mais peut s'en remettre à la coutume, quitte à intervenir en cas de conflit." 3

1 Ibid., pages 119-121.

2 Ibidem.

3 S.U.C.O.-C.R.A.D., op cit., page 121. 
Dans les villages qui possèdent une ou plusieurs rizières, elles appartiennent généralement à l'ensemble du village, tout comme les terres ordinaires. Les travaux et les corvées de réparation, d'entretien ou de préparation des rizières sont collectifs et, souvent, impliquent plus d'un village; dans ce cas, la rizière appartient à l'ensemble de ces villages qui sont généralement peuplés de la même ethnie.

Ainsi, comme le souligne Diana Lima Handem, chez les Balantes, les moyens de production sont selon leur mode d'utilisation, soit individuels comme dans le cas des instruments qui constituent le prolongement de la main, soit collectifs comme les digues et les canaux de drainage. ${ }^{1}$ Quant à la terre, elle est collective bien qu'il importe de relever la nuance qu'elle appartient à ceux qui la cultivent bien qu'elle soit propriété collective:

"La terre, objet de travail inaliénable, appartient aux villageois et aux familles qui la travaillent. Elle ne peut être ni vendue, ni loué par un métayage ou par fermage. Ici la terre est synonyme de survie du groupe, et par là signifie son identité par rapport aux autres groupes. Elle souligne l'autorité interne de son chef et la reconnaissance de cette autorité par l'extérieur. La terre apporte la reconnaissance de la légitimité du groupe par les vivants mais aussi par les Ancêtres et les Esprits, dans la mesure où celui qui l'acquiert et la fait fructifier établit un pacte protecteur avec ces entités religieuses." 2

Signe des temps, mais aussi relativisation nécessaire à tout, il nous aura été permis de constater la présence à Bolama d'un Balante, sorte d'homme d'affaires local, qui loue l'utilisation de ses terres contre du riz aux personnes sans terre. Ce cas constitue cependant une exception à Bolama, et l'on doit se garder de généraliser.

\subsubsection{Unité familiale d'exploitation}

1 Diana Lima Handem, Nature et fonctionnement du pouvoir chez les Balanta Brassa. Bissau, I.N.E.P., 1986, page 21 .

2 Ibidem. 
Au niveau de l'exploitation de la terre, nous privilégierons au terme famille, celui plus technique d'unité familiale d'exploitation ou U.F.E., qui peut se comparer à celui d'unité de production agricole, U.P.A., ${ }^{1}$ mais qui a un sens plus large que l'usage généralement admis que les Occidentaux attribuent à la famille, et moins large que celui bissau-guinéen identifiant couramment ethnie à famille:

"Nous entendons par unité familiale d'exploitation le groupe de parents et protégés qui, sous la direction d'un chef de famille, travaillent ensemble les produits essentiels; ce groupe constitue une unité qui peut elle-même être mono ou pluricellulaire, c'est-à-dire comprendre un ou plusieurs foyers (monogames ou polygames), ou encore se partager en petits groupes rassemblés autour de chacune des femmes d'un chef de famille polygame.

L'unité familiale d'exploitation constitue la cellule économique de base. Ceci n'empêche nullement les membres actifs de ce groupe d'avoir, en plus des tâches communes, des activités à revenus monétaires personnelles et l'autonomie des dépenses relativement à ces revenus individuels." 2

L'unité familiale d'exploitation type à Bolama se compose en moyenne de 6,6 personnes, soit 3,2 hommes et 3,4 femmes. On estime qu'au sein de chaque U.F.E., 2,2 personnes sont effectivement actives, 1,0 homme et 1,2 femme. 3

1 Simone Payeur définit lU.P.A. comme le groupe de "personnes cultivant ensemble au moins un champ commun (généralement un champ de céréales) et tirant leur nourriture d'un grenier commun" (Simone Payeur, Enquête en milieu rural,.1983, document No. 1, s.1., Projet de Développement Rural Zone 2, Service Evaluation, avril 1983, page 2).

2 Anne-Marie Hochet, Paysanneries en attente: Guinée-Bissau. Dakar, Environnement Africain, Série Etudes et Recherches, No. 79-80, janvier-février 1983, pages 48-49.

3 Jacques Bitard, op. cit, page 193. 
La superficie moyenne des exploitations couvre 1,3 hectare réparti comme suit: ${ }^{1}$

- riziculture: 0,47 ha;

- maïs: 0,07 ha;

- sorgho: 0,14 ha;

- mil: 0,09 ha;

- arachide: 0,38 ha;

- manioc: 0,1 ha;

- autres: 0,05 ha.

Enfin, l'unité familiale d'exploitation moyenne possède cinq animaux et 5,3 volailles à Bolama. ${ }^{2}$

\subsubsection{La riziculture}

La principale activité agricole en Guinée-bissau et en l'occurrence à Bolama, est la céréaliculture, tout particulièrement celle du riz, base de l'alimentation bissau-guinéenne. D'ailleurs il est courant d'entendre les bissau-guinéens dire qu'un repas sans riz n'est pas un repas...

Le riz se cultive à Bolama suivant trois méthodes: ${ }^{3}$

- la riziculture sur brûlis;

- la riziculture en rizière d'eau douce sur sol hydromorphe; et,

- la riziculture en rizière d'eau salée sur sol halomorphe.

\footnotetext{
1 Ibidem.

2 Ibidem.

3 S.U.C.O.-C.R.A.D., op.cit., page 112.
} 
Sur l'île de Bolama, 50 hectares sont consacrés à la riziculture sur brûlis, 28 à la riziculture d'eau douce et 296 à celle d'eau salé. ${ }^{1}$

La riziculture sur brûlis s'effectue sur des terrains en brousse, qui ont déjà été utilisés par les mêmes unités familiales d'exploitation par le passé, et dont la jachère est arrivée à maturité. On défriche, brûle puis sème ce champ ${ }^{2}$. Cette technique de culture de riz, appelé pluvial puisque seule la pluie alimente en eau la rizière qui ne s'inonde pas et ne conserve pas ainsi son eau, est pratiqué par la majorité des ethnies à Bolama. ${ }^{3}$

La riziculture en rizières d'eau salée est une spécialité balante, ethnie qui a initié à cette méthode d'autres ethnies dont les Manjaques, les Pepels, les Mandingues et les Bijagos. ${ }^{4}$ Cette technique, aussi surnommée riziculture de mangroves, demande une importante maind'oeuvre et exige des moyens impressionnants puisque la rizière est aménagée sur une partie de l'estran que l'on protège de la marée par la construction d'une digue. ${ }^{5}$ Cette méthode de culture en est une dite "inondée", une certaine quantité d'eau recouvrant constamment la rizière. La référence à l'eau salée ne réside pas dans le fait que le riz pousse dans l'eau salée, ce qui n'est pas le cas, mais plutôt, en ce que l'on inonde d'eau salée, après quelques années de culture, la rizière afin de lutter contre les mauvaises herbes et les parasites. A Bolama, la riziculture en eau salée correspond à la plus grande superficie identifiée. Cependant, dans les faits, la majorité de la surface identifiée à ce type de riziculture est abandonnée pour diverses

${ }_{1}$ Ibid., page 117.

2 Ibid., pages 128-130.

3 Brigitte Boies, Alain Rousseau, Guide pour l'analyse des systèmes de production: archipel Bolama-Bijagos. Guinée-Bissau, op. cit, 26 pages + annexes.

4 S.U.C.O.-C.R.A.D., op. cit., page 126.

5 Ibid., pages 130-132. 
raisons dont la rupture de la digue et le manque de moyens tant techniques que humains pour la réparer. ${ }^{1}$

La riziculture en eau douce, également appelée riziculture de bas-fonds, est elle-aussi une culture inondée. On la pratique sur des bas-fonds qui ont la particularité de s'inonder en saison des pluies, la construction de petites digues permettant la rétention de l'eau de pluie. ${ }^{2}$ Cette méthode de culture n'est pas courante à Bolama et seuls quelques villages la pratiquent: Lala Mancanha (peuplé de Mancagnes), Bolama de Baixo (ethnie bijago) et Uato Mandinga (ethnie mandingue). ${ }^{3}$

En matière de production rizicole, la présence d'un centre urbain sur l'île de Bolama, combinée au caractère d'autoconsommation que l'on donne à la culture du riz dans les villages qui ne sont souvent même pas autosuffisants en la matière, font que l'île est nettement déficitaire et ne nourrit pas sa population. Ainsi, en 1980, la production totale de riz à Bolama se chiffrait à 271,4 tonnes alors que la consommation estimée était de 651,3 tonnes, ce qui représente un déficit rizicole de 439,3 tonnes, ${ }^{4}$ soit $61,9 \%$ de plus que la production totale de l'île. L'étude S.U.C.O.-C.R.A.D. ne s'étonne pas de l'existence d'un tel déficit à Bolama puisque sa population urbaine est, quantitativement parlant, importante, et qu'à l'époque de l'étude, les sites rizicoles de l'île étaient dans un état déplorable. ${ }^{5}$

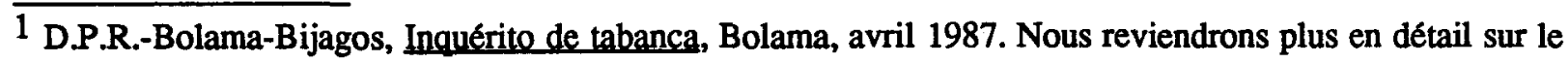
problème d'abandon des rizières au chapitre six.

2 S.U.C.O.-C.R.A.D., op. cit., page 126.

3 Brigitte Boies, Alain Rousseau, op, cit., page 21.

4 S.U.C.O.-C.R.A.D., op. cit., page 159.

5 Ibid., page 158. 
En saison des pluies, en plus de la culture du riz, on s'adonne à celle d'autres céréales comme le maïs et le sorgho, les légumineuses (arachide, haricot, pois de terre) et les tubercules (manioc et patate douce). 1

\subsubsection{Le maraîchage}

Après la saison des pluies, une fois le riz récolté, séché, vanné et entreposé, les grands travaux agricoles sont, pour les hommes, terminés, si ce n'est pour la réfection ou la préparation des rizières et des champs de riz pluvial. Les femmes, quant à elles, peuvent s'adonner, à la saison sèche, aux cultures maraîchères: laitue, tomates, oignons, aubergines, gombos, etc. Anne-Marie Hochet signale l'existence de cette activité qui, comme nous le verrons lors de l'étude de la division sexuelle des tâches, pour bien des femmes, répond d'abord à un besoin monétaire:

"C'est précisément en pays Pepel et dans les villages presque sans terroir de l'ilha de Bolama que le maraî́chage est le plus développé, mais il est également pratiqué, à moindre échelle, dans les autres régions (et pourrait l'être beaucoup plus qu'il ne l'est!). Nous notons que les femmes ont tendance à s'orienter vers le maraîchage à but lucratif, et notamment la culture de la tomate." 2

Pour le maraîchage comme pour la céréaliculture, la disponibilité et l'approvisionnement en semences sont toujours problématiques en Guinée-Bissau. C'est pourquoi, à Bolama, le Projet de Développement Agricole Bolama-Bijagos procéda à la distribution de semences et ce, tout particulièrement à partir de la saison 1987-1988. Cette distribution de semences de légumes, effectuée sous forme de vente afin de ne pas aliéner les personnes concernées, aura permis, lors d'une enquête sur la commercialisation des produits

1 ibid, pages 137-138.

2 Anne-Marie Hochet, op. cit. page 115. 
maraîchers, ${ }^{1}$ le suivi régulier d'un échantillon d'une vingtaine de maraîchères. Ce suivi aura débouché entre autres sur la préparation d'un calendrier des activités liées au maraîchage, calendrier où l'on peut voir que la saison marâichère débute peu après la saison des pluies, lorsque la période de récolte de riz commence à battre son plein (voir au Tableau 3.1). Ce chevauchement des activités de récolte de riz et de lancement de la campagne maraîchère, contraint les maraîchères à retarder la préparation de leurs jardins jusqu'à ce que la récolte du riz soit terminée, ce qui pose une contrainte de taille au maraîchage, celui-ci ne pouvant commencer que tard en saison. Ce retard a pour effet d'écourter la saison végétative, les puits s'asséchant peu à peu au fur et à mesure de l'avancement de la saison sèche, ce qui rend toute culture souvent impossible vers les mois de mars et avril. A partir de mars, la cueillette du cajou commence, et on peut comprendre que le maraîchage à Bolama est une activité complémentaire soumise à bien d'autres impératifs.

Au moment de la compilation des résultats de la distribution de semences, à la fin de décembre 1987, 16 villages et 87 personnes avaient été touchées par le programme. Ces 87 personnes étaient toutes des femmes sauf un homme, d'ethnie bijago. Les maraîchères appartenaient, pour la grande majorité d'entre elles $(85,14 \%)$, à l'ethnie mancagne, le reste se répartissant entre Manjaques, Pepels, Beafadas, Mandingues et Foulas. Quant au choix de semences, l'intérêt allait aux oignons avec $68,67 \%$ des maraîchères qui en avaient achetées, aux tomates $(50,6 \%$ des maraîchères), à la laitue $(20,48 \%)$, aux poivrons $(19,28 \%)$, aux choux $(13,25 \%)$, aux gombos $(8,43 \%)$ et aux carottes $(3,62 \%) .^{2}$

Les maraîchères se sont avérées les seules responsables de leur activité, leur mari ou d'autres hommes n'intervenant que pour la réalisation de tâches généralement masculines

\footnotetext{
1 Brigitte Boies, Alain Rousseau, Enquête sur la commercialisation des oroduits maraîchers, Bolama, Projet de Développement Agricole Bolama-Bijagos, C.E.C.I., mai 1988, 80 pages.

2 Ibid., pages 16-17.
} 
TABLEAU 3.2

Calendrier des activités maraîchères

Ile de Bolama (1)

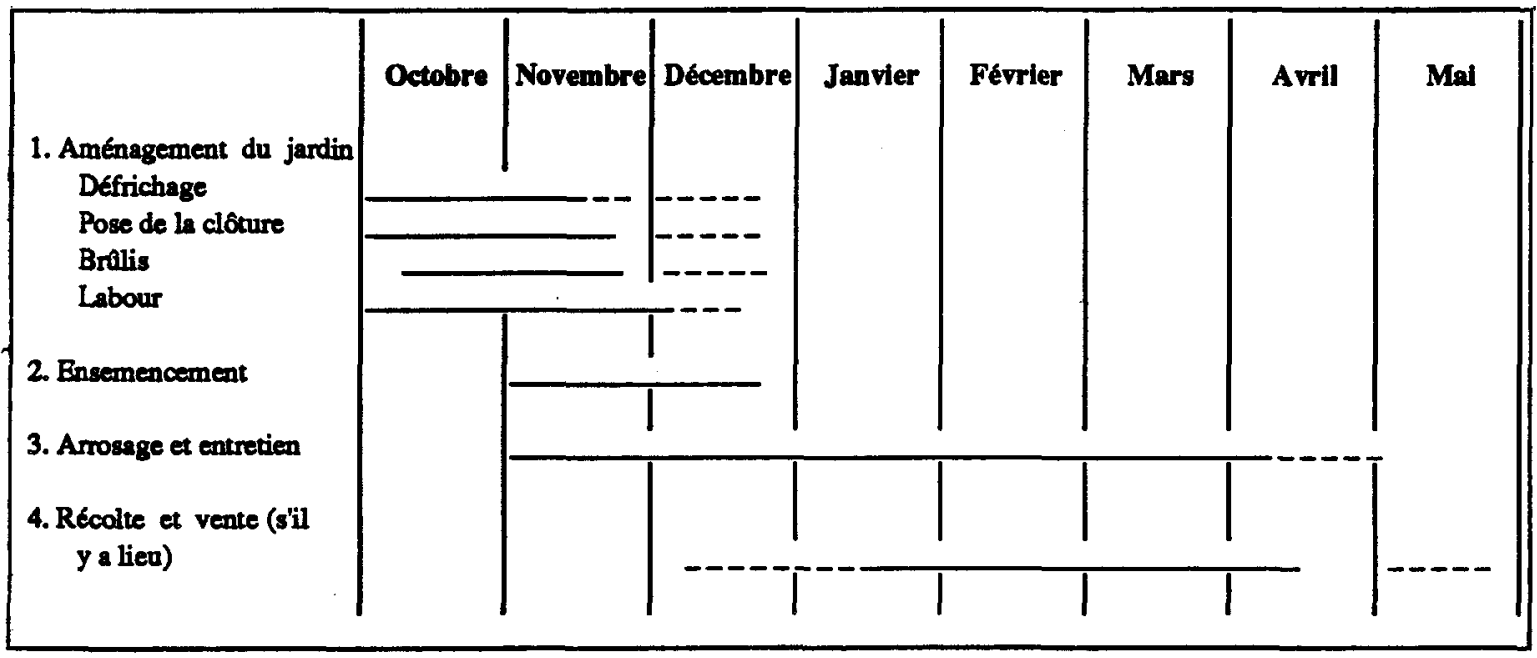

(1): Les traits continus (__ ) indiquent les mois où se déroule l'activité; les traits pointillés (_-_) sont utilisés lorsque l'activité est intermittente.

Source: BOIES, Brigitte, ROUSSEAU, Alain, Guide pour l'analyse des systèmes de production; archipel Bolama-Bijagos, Guinée-Bissau, Projet de Développement Agricole Bolama-Bijagos, Bolama, C.E.C.I., mai 1988, Annexe. 
telles qu'écimer les palmiers devant servir à construire les clôtures pour protéger le jardin des animaux domestiques ou creuser un puits, comme le montre le Tableau 3.3. Cependant, plusieurs d'entre elles partageaient leur travail et ses résultats avec une de leur (ou leur seule) "co-Épouse" (autre femme de leur mari), tout en se faisant aider par certains de leurs enfants. ${ }^{1}$

Alors que la culture du riz monopolise l'ensemble des ressources humaines de l'unité familiale d'exploitation (hommes, femmes et enfants), et que sa production vise avant tout l'autoconsommation, le maraîchage n'implique que la femme, et son but est généralement la vente de la production:

"Nous avons constaté que les revenus tirés des jardins appartiennent entièrement à la maraîchère qui l'exploite, sans que son mari n'y ait droit de regard, quelles que soient l'ethnie ou la religion, exception faite d'un homme Bijago qui, fidèle à ses traditions ethniques, doit remettre la totalité de ses revenus à sa femme." 2

Comme nous le verrons lors de considérations ultérieures sur le commerce, la culture marâichère s'adresse avant tout à fournir le marché urbain de Bolama dont, pour des raisons souvent liées aux prix pratiqués et au pouvoir d'achat des populations locales plutôt qu'à la seule insuffisance quantitative de la production, elle ne réussit pas à combler l'ensemble des besoins. Il est vrai que la principale préoccupation quotidienne de la population urbaine de Bolama, qu'elle dispose de sources de revenu ou non, concerne la recherche de nourriture. Ce souci journalier s'explique autant par l'insuffisance de l'offre de nourriture que par son coût élevé. Ainsi, la population urbaine est-elle constamment confrontée à cette difficulté de satisfaire ses besoins alimentaires et, généralement, les différents auteurs traitant du sujet n'ont abordé le problème que sous l'angle le plus facilement observable, l'absence d'offre adéquate, pour ignorer à toutes fins utiles les problèmes relatifs au faible pouvoir d'achat

\footnotetext{
${ }_{1}^{1}$ Ibid., pages 67-69.

2 Ibid, page 69.
} 
TABLEAU 3.3

Répartition des tâches maraîchères

selon l'âge et le sexe

Ile de Bolama (1)

\begin{tabular}{|l|r|}
\hline \multicolumn{1}{|c|}{ Actlvité } & Participants \\
\hline 1. Defrichage & $\mathrm{F}, \mathrm{H}$ \\
2. Confection et installation & \\
de la clotrure: & \\
$\quad$ couper le bois & $\mathrm{F}$ \\
$\quad$ trimer les palmiers du bois & $\mathrm{H}$ \\
$\quad$ installation des pieux & $\mathrm{F}$ \\
$\quad$ palmier aux pieux & $\mathrm{F}$ \\
3. Bralis & $\mathrm{H}$ \\
4. Labour & $\mathrm{F}, \mathrm{H}$ \\
5. Pose de l'engrais & $\mathrm{F}, \mathrm{H}$ \\
6. Semis et repiquage & $\mathrm{F}$ \\
7. Arrosage et entretien & $\mathrm{F}$ \\
8. Creusage des puits & $\mathrm{F}, \mathrm{E}$ \\
9. Recolte & $\mathrm{H}+\mathrm{F}$ \\
10. Séchage et transformation & $\mathrm{F}$ \\
(lorsqu'il y a lieu) & $\mathrm{F}$ \\
11. Vente et transport & $\mathrm{F}$ \\
\hline
\end{tabular}

(1): H: hommes; F: femmes; E: enfants. Le signe + signifie que les participants travaillent ensemble; la virgule (,) précédant une lettre, indique la participation circonstancielle du sujet suivant la virgule.

Source: BOIES, Brigitte, ROUSSEAU, Alain, Guide pour l'analyse des systèmes de production: archipel Bolama-Bijagos, Guinée-Bissau, Projet de Développement Agricole Bolama-Bijagos, Bolama, C.E.C.I., mai 1988, Annexe.

bolamien. Il importe cependant de relativiser cette prise de position puisque, il y a quelques années, et c'est sans doute ce qui explique le raisonnement des auteurs jusqu'ici, avant que 
l'inflation ne commence à affecter le pays, la population avait plutôt, comme principale préoccupation, non de rationaliser ses dépenses en fonction d'un budget limité, mais de trouver une façon de dépenser cet argent.

Plusieurs exemples, tout au long du mémoire, nous convaincront cependant de la complexité du problème que l'on ne peut seulement relier aux carences commerciales mais aussi aux prix élevés et aux habitudes alimentaires propres de la population qui ne possède pas un régime alimentaire varié. C'est ainsi que sa consommation de légumes, par exemple, se réduit à sa plus simple expression, héritage sans doute de l'économie de subsistance où la communauté, plutôt que de rechercher le régime alimentaire le plus équilibré et le plus diversifié possible, se contente de la consommation d'un aliment de base constituant "l'essentiel d'un régime alimentaire assez monotone mais suffisant eu égard à l'activité économique déployée par les intéressés". ${ }^{1}$ On comprendra dès lors que même une offre suffisante et diversifiée, combinée à un pouvoir d'achat adéquat, ne peuvent garantir le succès de la commercialisation d'une production maraîchère diversifiée, l'habitude de la consommation quasi-exclusive de riz agrémenté de citron, de piment, d'huile et éventuellement de poisson ou de viande étant trop profondément ancrée dans les moeurs locales, et n'étant substituée par d'autres aliments que lors d'éventuelles périodes de disettes.

Quoiqu'il en soit, la précarité alimentaire de l'île de Bolama reste toujours présente tout comme à l'époque de l'étude S.U.C.O.-C.R.A.D. qui dressait alors le bilan suivant sur la situation alimentaire de Bolama:

"Le haricot, le pois de terre, l'arachide et, en proportion plus réduite, le manioc et la patate douce servent d'aliments d'appoint durant l'année et de substitue au riz lorsque celui-ci est épuisé, sans toutefois combler toute la période de soudure. De plus, les cultures maraîchères pratiquées par les femmes à Bolama ne satisfont pas à la demande du marché local. On peut affirmer en définitive que les

$\overline{1}$ Robert Badouin, Economie nurale, Paris, Librairie Armand Colin, 1971, page 27. 
productions de légumes sont insuffisantes pour combler les besoins alimentaires de la population.

Toutefois, il faut noter que même si globalement la région est déficitaire pour sa production rizicole et que la période de soudure peut parfois s'étendre de juin à octobre, c'est surtout sur l'île de Bolama que les pénuries alimentaires sont les plus marquées. Sa population urbaine et le faible rendement des productions agricoles, qui n'arrivent même pas à combler les besoins ruraux, la rendent fortement dépendante des importations. Dans l'archipel, en période de soudure, la population Bijagos trouve dans la nature quantité d'aliments riches en protéines, vitamines et lipides pouvant assurer sa survie: fruits sylvestres, coeurs de palmiers, huile de palme, etc.." 1

\subsubsection{Les fermes d'Etat}

Afin de pallier aux déficiences alimentaires de Bolama, le Ministère du Développement Rural, le M.D.R., a converti en fermes d'Etat, d'anciennes plantations portugaises. Ayant comme objectifs l'appui aux producteurs locaux, notamment par la distribution de semences et de soins phyto-sanitaires et vétérinaires, l'approvisionnement de Bolama et de l'usine Titina Sila, la vulgarisation technique et la diversification des espèces cultivées, la ferme d'Etat de Bolama était inopérante à l'époque de l'étude S.U.C.O.-C.R.A.D. ${ }^{2}$. Lors de notre séjour, la ferme fonctionnait officiellement. Cependant, le Projet de Développement Agricole Bolama-Bijagos qui devait appuyer celle-ci, a abandonné tout espoir de la revitaliser vu les nombreuses contraintes, dont le manque d'eau douce sur le territoire de la ferme et le manque flagrant de productivité de ses employés, fonctionnaires du Ministère du Développement Rural. S.U.C.O.-C.R.A..D avait d'ailleurs déjà à l'époque, reconnu la vétusté très avancée des équipements utilisés et la détérioration relevant d'un quasi-abandon des plantations. Cependant, l'étude avait surtout été frappée par l'incompétence du personnel et la similitude entre les orientations de la ferme d'Etat et celles des colons portugais, ce qui ne pouvait

\footnotetext{
1 S.U.C.O.-C.R.A.D., op cit., page 160.

2 Ibid. pages 171-175.
} 
qu'avoir un effet négatif sur les relations entre la ferme et les populations locales. ${ }^{1}$ D'ailleurs faut-il noter qu'en 1984, on évaluait la productivité mensuelle de chaque travailleur de la ferme à 19 P.G. alors que son salaire était de 1475 P.G. ${ }^{2}$.

Notons cependant que les diverses plantations de la ferme d'Etat à Bolama, qui présentent actuellement un état d'abandon presque général, regroupent les espèces suivantes: cocotiers, kolatiers, citronniers, orangers, mandariniers, anacardiers, ananas, bananiers et légumes divers. ${ }^{3}$ Les productions sont parfois écoulées sur le marché local. Par contre, outre les cajous destinés à l'usine de compote de Bolama et les cocotiers réservés à l'exportation, de nombreux produits, surtout les produits rares tels les ananas, ne font pas état d'une commercialisation générale mais sont plutôt dirigés vers les besoins des notables locaux par des circuits de distributions souterrains hors de portée de la population...

\subsubsection{Elevage}

Soulignons enfin la présence d'élevage dans l'île. L'élevage, qui n'est pas intẹsif, répond essentiellement aux besoins d'autoconsommation des populations rurales et urbaines. Ainsi, chaque unité familiale d'exploitation possède une basse-cour composée de poules, canards, chèvres et porcs. L'élevage bovin n'est pas répandu en ville mais, en campagne, il est pratiqué couramment, surtout chez les Foulas, ethnie de tradition pastorale:

"A Bolama, la pratique de l'élevage est différente de celle de l'archipel du fait que les bovins sont gardés en troupeau et leur pâturage contrôlé. Ainsi dans le village de Uato Fula, les paysans vont conduire leurs bovins dans des champs. Le soir, ils peuvent y être gardés ou ramenés au village. L'insémination naturelle

1 Ibid., page 174.

2 Hugues Albert, Serge Blais, Marthe Côté, Pierre Thiran, Projet de développement agricole dans les îles Bolama-Bijagos. République de Guinée-Bissau, s.l., Programme Eudes et Projets de Développement Rural, C.E.C.I., novembre 1984, Tome I, Etude de base proposition de projet, page 88.

3 S.U.C.O.-C.R.A.D., op.cit, page 172. 
est contrôlée et la traite est une pratique courante et lucrative car on y vend le lait à des habitants de la ville de Bolama." 1

\subsection{La cueillette}

Les activités de cueillette sont réservées aux femmes, accompagnées des enfants, hormis la cueillette des régimes de palme, effectuée par les hommes provenant surtout des ethnies pepel et bijago.

La cueillette répond à deux besoins particuliers. Dans le cas des produits directement comestibles (crustacés, mollusques, coeurs de palmiers et pommes de cajou), la cueillette vise avant tout la satisfaction des besoins alimentaires des populations en période de soudure. Par contre, en ce qui concerne la noix de cajou, classée au rang des exportations prioritaires du pays, la cueillette est fortement stimulée par la recherche d'argent ou de riz lorsque troquée, puisque cette pratique est courante chez les commerçants privés comme publics qui "achètent" les noix à la population.

A Bolama, les activités de cueillette sont surtout axées sur les fruits de l'anacardier, la pomme et la noix de cajou. Le jus de la pomme, très riche en vitamine $\mathrm{C}$ et en sucre, a la propriété de se transformer en alcool sous le seul effet de la chaleur. Cet alcool, appelé vin de cajou, est très prisé de la population animiste. On l'obtient en pressant les pommes de cajou et en laissant fermenter le jus ainsi obtenu qui ne tarde pas, en quelques jours, à se transformer en alcool.

Parallèlement à cela, la noix de cajou est consommée pour une petite part du total récolté, grillée. La majeure partie de la cueillette de noix est réservée, comme nous l'avons

\footnotetext{
${ }^{1}$ Ibid., page 162.
} 
déjà précisé, à l'exportation. En effet, et au même titre que la noix de coco et l'arachide, la noix de cajou fait partie des produits d'exportation visant à fournir le pays en devises étrangères. Jusqu'en 1987, l'achat et l'exportation des noix de cajou étaient réservés aux deux sociétés commerciales d'Etat, la Socomin et les Armazens do Povo. A partir de cette date, les commerçants privés purent prendre part à la récolte de cajou, mais non à son exportation, encore réservée aux sociétés d'Etat. ${ }^{1}$

Cueillie vers la fin de la saison sèche (de mars à mai), en pleine période de soudure, la noix de cajou revêt une importance capitale pour une population chez qui le déficit alimentaire est chronique, à un point tel que les gens affluent de nombreux coins du pays pour participer à la récolte. ${ }^{2}$ Le gouvernement a fixé à partir de 1987 les termes du troc à deux kilos de noix contre un kilo de riz alors qu'en 1986 , les deux produits s'échangeaient à poids égal. ${ }^{3} \mathrm{Du}$ côté des prix officiels, le prix était fixé à 175 P.G. le kilo en 1987. En 1988, le gouvernement laisse jouer les lois de la concurrence entre les commerçants et leur donne la liberté de fixer les prix à partir d'un prix plancher de 200 P.G. le kilo de noix. ${ }^{4}$ On a vu, à ce moment, les prix varier à Bolama entre 250 et 275 P.G. le kilo.

Bolama exporte donc les noix de cajou qu'elle ne consomme pas et cette exportation semble être en augmentation. Ainsi, l'entreprise Socomin exportait de l'île de Bolama, en 1977, pour un peu plus de neuf tonnes. En 1978, ce chiffre baissait à quatre tonnes pour

1 Entrevue avec El Hadj Camara, directeur pour la région Bolama-Bijagos des Armazens do Povo, Bolama, 30 mai 1988.

2 Brigitte Boies, Alain Rousseau, Guide pour l'analyse des systèmes de production: archipel Bolama-Bijagos. Guinée-Bissau, op.cit., page 6.

3 Philippe de Braconier, Elizabeth Drory, Rapport de mission en Guinée-Bissau du 3 au 13 avril 1987, Huy, Iles de Paix, 12 mai 1987, page 2.

4 Entrevue avec El Hadj Camara, op. cit. 
ensuite remonter à près de 120 tonnes en $1979{ }^{1}$ et à plus de 93 tonnes en $1984 .^{2}$ Les Armazens do Povo, pour leur part, exportent de plus grandes quantités de noix de cajou. Alors que la Socomin exportait 93 tonnes de noix en 1984 par exemple, ceux-ci exportaient 256 tonnes, 3 ce qui portait le total des exportations de l'île à 349 tonnes. Au niveau national, l'exportation de la noix de cajou de la région Bolama-Bijagos comptait pour $10 \%$ du total national exporté. ${ }^{4}$ La majorité de la production régionale provient de l'île de Bolama où le cajou est grandement cultivé comme l'atteste le fait que la production exportée à partir de Bolama par la Socomin, de 1977 à 1979, représentait plus de 95\% du total de la région. 5 Enfin, la campagne de 1987, se soldait par une exportation de 550 tonnes de cajou à partir de Bolama 6 et l'on peut supposer que Bolama exporte maintenant environ 600 tonnes de noix de cajou par année. 7

L'importance que revêt la cueillette de cajou aux yeux de la population est telle que toute autre activité qui se déroulerait à la même époque serait voué à connaître un succès des plus limités. L'activité de cueillette touche tous les villages de lî̂le comme nous l'a démontré l'enquête de tabanca. La transformation du vin de cajou s'effectue, pour sa part, dans la totalité des villages de religion animiste. Lors de notre enquête, nous avons pu constater que 20 villages sur 25 produisaient du vin de cajou. Un manque chronique de récipients et, assurément, des méthodes de conservation et de contrôle bactériologique déficientes, limitent en grande partie cette activité à la consommation locale, plutôt qu'à l'exportation, bien que

1 Socomin, Service de contrôle, Bissau, 15/01/80.

2 SCET-AGRI, Etude des prix et incitations aux producteurs nuraux, République de Guinée-Bissau, MICEPCI, janvier 1986, page 75 .

3 Ibidem.

4 Ibidem.

5 Socomin, op cit., page 1, compilation interne.

6 Entrevue avec El Hadj Camara, op. cit.

7 Hannes Stegemann, Ile de Paix de Bolama rapport de mission à Bolama. 03/04/87-23/05/87, Huy, Iles de Paix, 1987, page 16. 
certains particuliers pouvant disposer de fûts, en exportent vers Bissau où le prix de vente est nettement plus élevé qu'à Bolama.

Le fruit du palmier est lui aussi cueilli mais, sa cueillette, tout comme la collecte du vin de palme, est une activité essentiellement masculine. Par contre, la transformation de ces fruits en huile, qui relève du travail de la femme et des enfants, était pratiquée, à Bolama, selon notre enquête par dix villages, en majorité situés dans la partie sud de l'île où les palmiers sont plus abondants.

Enfin, selon la période de l'année, les femmes et les enfants s'adonneront à la cueillette de fruits, de mollusques et de crustacés pour compléter la diète alimentaire quotidienne de l'unité familiale d'exploitation.

L'île de Bolama compte un certain nombre de plantations de cocotiers qui datent du temps des Portugais, et l'étude S.U.C.O.-C.R.A.D. évaluait le nombre d'arbres de ces anciennes plantations à $9887 .{ }^{1}$ Comme le cocotier n'est pas une espèce indigène au pays, la population n'est pas familière avec ses nombreuses possibilités d'exploitation. De plus, le régime foncier pratiqué pour ces plantations, celles-ci appartenant au comité d'Etat régional, ne permet pas l'exploitation populaire mais vise l'exportation. La cueillette de la noix de coco est de ce fait, marginale, bien que la population bolamienne en fasse tout de même une certaine utilisation, qui se limite à la consommation de la noix crue et à la préparation de desserts et de friandises. ${ }^{2}$

1 S.U.C.O.-C.R.A.D., op. cit, page 311.

2 Ibid., pages 307-312. 


\subsection{La pêche}

La riziculture, comme nous l'avons vu, est l'activité dominante des habitants de Bolama et elle est importante au point de voir toutes les autres occupations lui être subordonnées. La pêche n'y fait pas exception, même chez une population insulaire, entourée d'eaux riches en poissons comestibles. ${ }^{1}$ Par conséquent, à Bolama, la pêche sera pratiquée surtout sur une base artisanale, tant du point de vue des techniques et de l'équipement employés, que de la finalité de cette pêche, l'autoconsommation. ${ }^{2}$ Une ethnie musulmane originaire du Sénégal et de la Gambie, l'ethnie nhominca (appelée sérère au Sénégal), pratique cependant la pêche à un niveau professionnel et vend ses prises. Les Nhomincas, installés en Guinée-Bissau depuis les années 1930, y ont apporté leurs méthodes et leur matériel de pêche (filets pouvant être immergés à différentes profondeurs, et pirogues). D'ailleurs leurs pirogues ont été, en Guinée-Bissau, baptisées du nom de l'ethnie. Celles-ci, contrairement aux pirogues locales qui sont taillées dans un tronc d'arbre et mues à rames, sont le fruit d'un travail de menuiserie les rendant plus massives et leur permettant d'être équipées d'un moteur hors-bord. 3

\subsubsection{La pêche artisanale}

La pêche artisanale vise d'abord la satisfaction des besoins de consommation de l'unité familiale d'exploitation du pêcheur ou de son village. Les surplus qui sont rares, les sorties étant uniquement effectuées lorsque le besoin se fait sentir, sont conservés suivant les

1 Ibid., page 269.

2 En ce qui concerne la chasse, notons qu'elle est très peu pratiquée malgré la présence de gazelles, la population alléguant l'impossibilité de se procurer des munitions pour expliquer la quasi-inexistance de cette activité. Quoiqu'il en soit, il arrive, mais très rarement, que l'on rencontre des chasseurs qui concentrent leurs activités sur le continent plutôt que sur l'île.

3 Raul Mendes Fernandes, "Nhomincas e Bidjogos, da pesca de "subsistência" a pesca "comercial" ", Soranda Revista de Estudos Guineenses, No. 4, julho 1987, Bissau, I.N.E.P., page 59. 
méthodes traditionnelles de conservation (poisson séché, poisson salé-séché, poisson fumé). ${ }^{1}$

La pêche se pratique soit à partir de pirogues, soit à gué, selon le type de matériel utilisé (harpon, ligne, filet rectangulaire, filet circulaire). ${ }^{2}$ L'enquête de tabanca a recensé 15 villages où l'on s'adonne à la pêche, et un total d'une cinquantaine de pêcheurs. Le nombre de canots, comme on peut le voir au Tableau 3.4, est très limité ( 21 canots), et plusieurs villages n'en ont pas, fait qui est sans doute surprenant si l'on considère le caractère insulaire du milieu. Comme nous le soulignons à plusieurs reprises, les productions artisanales sont, à Bolama encore plus qu'ailleurs en Guinée-Bissau, très peu développées et, depuis la guerre, on ne produit presque plus rien par manque de matériel et, un peu, par fatalisme. Au niveau de la construction de pirogue, le savoir se perd peu à peu et les personnes possédant les connaissances nécessaires à la construction de pirogues sont très rares ainsi que le démontre notre enquête qui n'a trouvé que quatre villages sur 25 où l'on connaissait ces méthodes de construction. De plus, les grands arbres servant à la confection de pirogues étant protégés par le gouvernement, les formalités nécessaires à l'octroi d'un permis de coupe découragent plus d'un constructeur potentiel.

Les méthodes employées pour ce genre de pêche permettent surtout la capture du mulet (environ 90\% des prises), un poisson de quatrième catégorie, bien que les eaux entourant lîle recèlent également les espèces suivantes: barbeau, barracuda, perche de mer, sole (classés poissons de première catégorie), capitaine (deuxième catégorie) et perche des tropiques (troisième catégorie). ${ }^{3}$ La compilation des principales techniques de pêche employées dans les 15 villages identifiés au Tableau 3.4 démontre que les pêcheurs ont surtout recours à 1 S.U.C.O.-C.R.A.D., Op. cit., pages 269-274.

2 Ibid., pages 272-273.

3 Ibid., page 270. 
TABLEAU 3.4

Nombre de pêcheurs et de canots Ile de Bolama

1987

\begin{tabular}{|c|c|c|}
\hline Village & $\begin{array}{l}\text { Nombre de } \\
\text { pêcheurs }\end{array}$ & $\begin{array}{l}\text { Nombre de } \\
\text { canots }\end{array}$ \\
\hline Aranja Vida/Caboupa & 1 & 1 \\
\hline Balanbaï (Ponta Isidoro) & 1 & 0 \\
\hline Bairro Achamada & 0 & 2 \\
\hline Bolama de Baixo & 11 & 2 \\
\hline Calidje & 4 & 2 \\
\hline Gâ Beafada & 8 & 7 \\
\hline Ga Moria & 1 & 0 \\
\hline Ga Nafa & 1 & $\underline{0}$ \\
\hline Lala & 4 & $\underline{0}$ \\
\hline Lala Balanta & 4 & 0 \\
\hline Luanda & 3 & $\underline{0}$ \\
\hline Madina & 4 & $\underline{0}$ \\
\hline Ponta Apili & 1 & 0 \\
\hline Ponta Bissau & 0 & 1 \\
\hline Ponta Doce II & 6 & $\underline{0}$ \\
\hline Pujunguto & 0 & 1 \\
\hline Uato Mandinga & 9 & 5 \\
\hline
\end{tabular}

Source: D.P.R.-Bolama-Bijagos, Inquérito de tabanca, Bolama, avril 1987. 
l'utilisation de filets flottants et de filets circulaires (huit villages ayant indiqué qu'ils pratiquaient ces méthodes), technique suivie de l'emploi de la ligne (deux villages) et du filet qui emprisonne le poisson dans un bras de mer lors du retrait de la mer sous l'effet de la marée (un village).

\subsubsection{La pêche professionnelle}

Lors de notre enquête nous avons compté une soixantaine de pêcheurs sur l'île de Bolama, ce qui en soit differe peu du diagnostic du secteur du Ministère du Plan en région qui évaluait ce nombre à 49 en $1986{ }^{1}$. Les chiffres different cependant selon les sources, au niveau de l'estimation du nombre de pêcheurs professionnels, c'est-à-dire ceux dont l'activité vise avant tout le dégagement de revenus monétaires (combinés ou non de troc), leur permettant d'être leur seule activité productive ou, du moins, leur principale. Il est à noter que Côté et Demers portent ce nombre à une quarantaine ${ }^{2}$ alors que les responsables d'Tle de Paix l'évaluent à une quinzaine de personnes pêchant régulièrement, dont huit Nhomincas. ${ }^{3}$ Leur nombre dépendra ainsi, on ne peut en douter, de la définition que l'on accordera au terme professionnel.

Un relevé effectué en 1988 par le Secrétariat d'Etat aux Pêches à Bolama, PescarteBolama, dénombrait jusqu'à 160 pêcheurs professionnels dans le secteur de Bolama (Bolama, São-João, île de Gallinhas)! Ce chiffre, surprenant en soi, s'explique aisément lorsque l'on pousse plus loin l'investigation et que l'on apprend que de nombreux noms

1 Teté João Baraï, Diagnóstico do sector de Bolama, Bolama, D.P.R.-Bolama-Bijagos, julho de 1986, page 20.

2 Marthe Côté, André Demers, Repères pour un développement rural intégré dans la région de Bolama. Guinée-Bissau, op, cit, pages 127-128.

3 Philippe de Braconier, Elizabeth Drory, Rapport de mission de juin 1985: prétude de l'tle de Paix de Bolama, Huy, lles de Paix, 1985, page 18. 
inscrits appartiennent soit à des personnes fictives, soit à des personnes décédées. En fait, le phénomène se comprend aisément lorsque l'on se réfère au contexte de ce recensement, associé à la venue d'un projet de pêche à Bolama. Bien souvent, qui dit projet en GuinéeBissau, dit aussi distribution de matériel gratuitement ou à peu de frais: on comprend ainsi que les enchères n'ont pu que monter, chacun voulant être reconnu comme pêcheur et pouvoir ainsi avoir accès à du matériel de pêche si rare en Guinée-Bissau.

Le registre que possède Pescarte-Bolama, sur les pêcheurs dotés d'une pirogue motorisée et ceux sur les prises mensuelles rapportées au projet sont de loin les meilleures sources d'information dont nous pouvons disposer. Ainsi ceux-ci recensent-ils 19 pêcheurs "nationaux" avec pirogue à moteur et 15 étrangers, Nhomincas, soit 34 pêcheurs équipés de pirogue à moteur. ${ }^{1}$ On compte 50 pirogues avec ou sans moteur, et le nombre de pêcheurs ayant effectivement travaillé et livré du poisson au projet Pescarte durant les mois de janvier, février et mars 1988 , est respectivement de 26,30 et $42 .{ }^{2}$

Nous pouvons supposer que de nombreuses pirogues n'étaient pas en service lors des trois mois dont fait état le relevé de Pescarte. En effet, en plus des difficultés propres aux problèmes d'approvisionnement qui touchent le pays, Bolama ne compte aucun dépôt de carburant. Il en découle que les pêcheurs doivent se rendre soit à Bissau, soit à Bubaque auprès d'un projet de pêche, pour y acheter huile et pétrole nécessaires au fonctionnement de leurs moteurs. Ceci nous porte à croire que le nombre de pêcheurs professionnels à Bolama dépasserait la quarantaine pour atteindre peut-être même la cinquantaine. De ce nombre, plusieurs habitent São-João, les autres demeurant à Bolama et les environs. Une

\footnotetext{
1 Pescarte, Relação nominal por pescadores com canoa à motor. Bolama, Secretaria de Estado das Pescas, 12 de abril de 1988.

2 Pescarte, Captura por espécie referente aos meses de janeiro. fevereiro e març 1988, Bolama, Secretaria de Estado das Pescas, 12 de abril de 1988, pages 1-3.
} 
confrontation de ces chiffres avec ceux obtenus lors de l'enquête de tabanca et le retrait des pêcheurs professionnels selon un critère de proximité géographique par rapport à Bolama, puisque seuls les pêcheurs vivant à proximité de Bolama peuvent venir y vendre leurs captures, nous permet de remarquer encore la présence, en plus des pêcheurs professionnels travaillant effectivement dans le cadre de Pescarte, de 40 à 50 pêcheurs traditionnels villageois, selon que l'on exclue ou non de ce nombre les pêcheurs de Bolama de Baixo qui sont malgré tout professionnels, qu'ils soient Nhomincas ou fassent partie de leurs équipage. Il nous apparait donc, à la lumière de nos recherches, qu'il pourrait y avoir sur l'île de Bolama, une centaine de pêcheurs répartis également entre professionnels et artisans. Les premiers sont équipés d'embarcations et se consacrent activement à la pêche afin de dégager des revenus monétaires. Les seconds possèdent ou non une pirogue, et considèrent la pêche comme une activité d'appoint à leur régime alimentaire.

Les pêcheurs nationaux sont habituellement cultivateurs lorsqu'ils habitent en campagne. Leurs sorties en mer varieront donc selon les saison, une vingtaine par mois en saison sèche, soit huit mois par année, ${ }^{1}$ et beaucoup plus rarement sinon presque jamais en saison des pluies, temps de culture du riz. D'autres prétendent qu'elles sont de l'ordre de trois à quatre par semaines en saison sèche, avec des captures moyennes oscillant entre 200 et $300 \mathrm{~kg}$, alors qu'en saison des'pluies, elles s'espacent, diminuant à deux ou trois par semaine avec une moyenne de $100 \mathrm{~kg}$ de poisson par journée de travail. ${ }^{2}$ L'étude S.U.C.O.C.R.A.D. estime cependant la moyenne des prises à la moitié, soit environ $150 \mathrm{~kg}$ en saison sèche et $50 \mathrm{~kg}$ en saison des pluies. ${ }^{3}$ Cet écart pourrait peut-être s'expliquer par une éventuelle amélioration du matériel de pêche utilisé. On comprendra de plus, qu'en saison des

\footnotetext{
1 Philippe de Braconier, Elizabeth Drory, op. cit, page 19.

2 Teté João Barai, op.cit., page 20.

3 S.U.C.O.-C.R.A.D., op.cit. page 281.
} 
pluies, l'espacement des sorties repose dans les aléas du climat et dans les contraintes propres aux activités agricoles éventuelles du pêcheur.

En 1985, les captures totales pour Bolama furent estimées à $94040,5 \mathrm{~kg} .{ }^{1}$ Pendant les trois premiers mois de 1988, avec le fonctionnement de Pescarte, on a enregistré un total de $49153,5 \mathrm{~kg}$ de poisson, ${ }^{2}$ soit $52,3 \%$ de la production totale de 1985 . Si l'on se fie aux données de l'étude S.U.C.O.-C.R.A.D. selon lesquelles la demande de poisson est toujours supérieure à l'offre à Bolama, avec une demande quotidienne de $150 \mathrm{~kg},{ }^{3}$ il faudrait une offre annuelle de $54750 \mathrm{~kg}$, soit beaucoup moins que ce que l'on affirme pouvoir disposer. Nos observations démontrent que l'offre ne suffit toujours pas à la demande bolamienne, ce qui s'explique sans doute par l'irrégularité de l'arrivée de poisson, souvent espacée de plusieurs jours, et par les graves déficiences en matière de conservation et de distribution que connaît l'île. Parallèlement à l'irrégularité dans l'offre de poisson à Bolama, la majorité de la population urbaine ne peut se permettre à chaque jour, de consommer ce produit, de par son faible pouvoir d'achat.

Au niveau des pêcheurs, le manque de matériel est flagrant et, jusqu'à la venue du projet Pescarte en 1987, les pêcheurs ne pouvaient s'approvisionner qu'à Bubaque auprès d'un projet similaire. Cependant, les pêcheurs ne peuvent pour l'instant s'équiper en embarcation, ce qui constitue une grave entrave au développement de la pêche. Il est intéressant de noter qu'à Bolama, les pirogues sont propriétés privées mais peuvent être

1 Ministério do Desenvolvimento Rural e Pescas, Anuário estatístico 1985, Republica da Guiné-Bissau, Gabinete de Planeamento, abril de 1986, page 36.

2 PESCARTE, op cit., pages 1-3.

3 S.U.C.O.-C.R.A.D., op.cit, page 280. 
louées contre redevance en nature. ${ }^{1}$ Soulignons ainsi la pratique fréquente des Nhomincas d'avoir recours à l'engagement de salariés, souvent Bijagos, ${ }^{2}$ pour former leurs équipages.

\subsection{Les activités artisanales}

En plus des activités directement liées à l'agriculture et à la pêche, Bolama compte un secteur d'activités artisanales d'ailleurs très limité, ${ }^{3}$ comme le prouve le Tableau 3.5 donnant les résultats de l'enquête de tabanca.

Bien que l'on croit souvent que les activités de production artisanales aient été soit abandonnées, soit sérieusement ralenties à l'époque de la lutte au profit de l'effort de guerre, un rappel historique relativise cette affirmation. Comme on le sait, la Province de la GuinéeBissau n'était, aux yeux des Portugais, qu'une source de matières premières (bois, arachide, cuir, cire, etc) et qu'un marché de produits finis, ce qui fit du domaine des productions artisanales un secteur en voie de régression, nullement encouragé ou développé. ${ }^{4}$ Les premières années de l'indépendance virent, comme on le sait, le pays s'orienter vers des activités exportatrices, ce qui ne fit qu'accroître l'isolement et la marginalisation des activités artisanales face aux efforts de développement gouvernementaux. Tout ceci, combiné à un approvisionnement de plus en plus déficient en matières premières et en outils, entraîna le déclin progressif de ce type de production. 5 Ainsi, de nos jours,

\footnotetext{
1 Ibid., page 280.

2 Ibid., page 84.

3 Hannes Stegemann, Ile de Paix de Bolama, rapport de mission à Bolama. 03/04/87-23/05/87, op. cit., page 19.

4 Ministère de la Coordination Economique, du Plan et de la Coopération Internationale, La production populaire en Guinée-Bissau, Bissau, en collaboration avec OXFAM-Belgique, septembre 1985, Page 7.

5 Ibid., page 8.
} 
TABLEAU 3.5

Tableau de fréquence des activités de production selon le nombre de villages

Ile de Bolama

1987

\begin{tabular}{|l|r|}
\hline \multicolumn{1}{|c|}{ Type d'activité } & Nombre de villages \\
\hline Vin de cajou & 20 \\
\hline Sel & 15 \\
\hline Peche & 15 \\
\hline Construction de cases & 13 \\
\hline Fabrication de savon & 12 \\
\hline Vin de palme & 10 \\
\hline Huile de palme & 9 \\
\hline Fabrication de blocs & 8 \\
\hline Couture & 8 \\
\hline Vannerie & 7 \\
\hline Menuiserie & 5 \\
\hline Tissage & 4 \\
\hline Poterie & 4 \\
\hline Construction de canots & 4 \\
\hline Fumage de poisson & 2 \\
\hline Fabrication de bancs & 1 \\
\hline Artisanat du cuir & 1 \\
\hline
\end{tabular}

Source: D.P.R.-Bolama-Bijagos, Inquérito de tabanca, Bolama, avril 1987. 
"Les artisans, les producteurs et les productrices dans les villages en Guinée-Bissau souffrent d'abord et avant tout d'une pénurie générale et quasipermanente de fournitures et des moyens de production journaliers, due à la mauvaise organisation et à la faiblesse du système commercial.

A cause de ce manque de ressources, les artisans sont souvent condamnés au chômage technique, ou doivent se contenter d'une production partielle et intermittente. D'autres perdent beaucoup de temps à la recherche de la matière appropriée. Pour l'approvisionnement ils sont souvent livrés aux "Djilas", les commerçants ambulants, qui sont forcés d'appliquer des prix très élevés, conformément à la situation d'insécurité économique et financière dans laquelle ils travaillent. Certains artisans résolvent leurs problèmes en émigrant temporairement dans les pays voisins ou en y canalisant une partie de leur production pour pouvoir se procurer les outils indispensables." 1

Comme l'étude du commerce nous le démontrera, la pénurie de matières premières et d'équipement qui affecte la Guinée-Bissau, trouve, pour une bonne part, sa source dans le monopole qu'exerçait jusqu'à tout récemment le pays face à l'importation. Ce monopole est détenu par les sociétés publiques commerciales Socomin et Armazens do Povo. Un système de favoritisme en ce qui a trait à la distribution aux clients, un manque de productivité remarquable de la part des employés, et le manque de devises du pays, ont engendré cette situation de pénurie quasi-constante de tous les biens de consommation et de production.Ainsi dit-on, à propos des Armazens do Povo et de Socomin:

..."ces magasins disséminés partout dans le pays ont démontré leur faiblesse et leur vulnérabilité devant l'absence de devises, l'incompétence des gestionnaires ou la corruption. Et s'il n'est pas rare de trouver en pleine brousse des produits comme du porto ou du concentré de tomates, il est en revanche quasi impossible de se procurer les produits de première nécessité, savon, tissus, allumettes, etc., que recherchent les habitants des tabancas. Comment imaginer qu'un paysan se mette à produire pour le marché si le gain financier de sa vente ne lui permet pas d'avoir accès à des biens qui améliorent ses conditions de vie?" 2

Ce raisonnement ne s'applique pas seulement au paysan et à sa production mais aussi, on le comprend aisément, à tout producteur quel qu'il soit. De plus, comme le souligne le

1 Ministère de la Coordination Economique, du Plan et de la Coopération Internationale, op.cit. page 14.

2 Istvan Felkai, "Guinće-Bissau; tenir la promesse faite aux paysans ....", Le Monde Diplomatique avril 1983, page 15. 
Ministère du Plan, le monopole d'Etat sur la commercialisation et sur l'exportation de l'huile de palme, des noix palmistes, des noix de cajou et de coco, et de l'arachide, posait un frein certain aux activités de production artisanales puisqu'on ne pouvait ainsi ni vendre sa production, dans le cas de la production artisanale d'huile d'arachide par exemple, ni trouver les matières premières nécessaires en quantités suffisantes, pour la production de savon à partir d'huile de palme entre autres. Ajoutez à cela le manque de moyens de transport, l'absence de moyens de stockage efficients et les faiblesses techniques de la population dans l'exercice de ces activités, ${ }^{1}$ et vous obtiendrez un tableau assez évocateur de la situation du pays et de Bolama.

Le seul moyen, à ce moment, de contourner ces contraintes, reposait dans l'encadrement de ces activités de production par des projets de coopération qui pouvaient importer différents produits disponibles uniquement à l'étranger ou, au pays chez les quelques commerces opérant uniquement en devises étrangères. Avec la libéralisation du commerce permise ces dernières années, la fourniture de matériel par des projets étrangers devient de plus en plus une entrave au développement du commerce privé local, de par une certaine concurrence déloyale effectuée par ces projets qui peuvent vendre ce matériel à très bas prix. A Bolama cependant, la vente de matériel par des projets de coopération n'en est qu'à ses débuts: Ile de Paix ne fournit du matériel de menuiserie et de pêche que depuis quelques années, tout comme Pescarte avec son matériel de pêche ou le C.E.C.I. avec son petit outillage agricole disponible depuis 1986 . Tout ceci explique sans doute pour une bonne part, le faible développement des activités de production artisanales sur l'île. Quoiqu'il en soit, le mouvement de libéralisation amorcée récemment n'a, jusqu'à maintenant, eu que peu d'impact sur le développement des activités artisanales et on peut croire qu'il s'écoulera

\footnotetext{
${ }^{1}$ Ibid, page 18.
} 
encore plusieurs années avant que l'approvisionnement en matériel d'artisanat par les commerçants locaux ne soit effectif. En attendant, Bolama est dans une situation de quasiinexistence d'artisanat local.

Le travail du bois n'est pratiqué que par quelques menuisiers privés (au maximum cinq en ville) et dans cinq villages, si l'on excepte le projet Ile de Paix qui, dans son atelier, en forme une dizaine ${ }^{1}$. La fabrication de pirogues, pour sa part, n'est effectuée que dans quatre villages (Assomada, Calidje, Casseque, Luanda) où, dans l'un d'entre eux, Calidje, on fait de plus de petits bancs de bois. Quant à la sculpture sur bois, elle n'existe pas à Bolama.

Le travail du métal non plus ne se pratique pas à Bolama, que ce soit au niveau de la forge, de la ferblanterie, de la fonte d'aluminium, de l'orfevvrerie ou de l'armurerie et la construction mécanique.

Au niveau du travail du textile, on rencontre à Bolama le tissage et la couture, qui sont surtout pratiqués en ville.

En fait, on constate qu'à Bolama, les seules activités courantes sont celles qui ne nécessitent que l'utilisation de produits abondants comme la production du vin de cajou par exemple, ou encore qui répondent à un besoin de consommation essentiel (huile de palme, sel, savon, pêche, poterie, vannerie).

Quant à la construction de blocs de terre séchée, elle est nécessaire à la fabrication des cases qui sont en majorité faites à partir de matériaux locaux (terre, pisé, bois de rônier et paille) par le futur propriétaire aidé de membres de sa famille ou de sa communauté. ${ }^{2}$ Notons qu'il n'y a pas à Bolama d'entrepreneurs spécialisés dans la construction d'habitation et c'est

1 D.P.R.-Bolama-Bijagos, Inquérito dos projectos, Bolama, 9 de janeiro de 1988.

2 Ministère de la Coordination Economique, du Plan et de la Coopération Internationale, op. cit., page 6. 
donc à chacun que revient ce travail. Cependant, bien que les Foulas et les Mandingues possèdent des spécialistes en la matière ${ }^{1}$, nous n'avons pas pu le vérifier à Bolama.

\subsubsection{Spécialisations ethniques}

Nous avons maintes fois souligné l'existence de spécialités ethniques et il est important d'en tenir compte car l'expérience nous a appris que ces spécialisations revêtent souvent un caractère presqu'immunable chez ces ethnies, et que seule une prise en compte de l'héritage culturel de chacune permettra la mise en oeuvre d'un modèle de développement viable. Ainsi il convient de retenir que les Balantes sont avant tout cultivateurs de riz (spécialistes de la riziculture en eau salée), que les Bijagos cultivent le riz pluvial et le riz d'eau douce, en plus de s'adonner à la pêche et à la fabrication de l'huile de palme, que les Pepels, tout comme les Manjaques et les Beafadas, sont de grands cueilleurs de régimes de palme et qu'ils ont développé des techniques de tissage, de pêche artisanales et de forge. L'ethnie mancagne pratique le maraîchage alors que les Foulas font l'élevage et ont des castes professionnelles, tout comme les Mandingues qui sont peu enclins à la riziculture inondée mais plutôt à la culture du mil, de l'arachide et du riz pluvial. Enfin il faut noter la présence de deux ethnies plus marginales, les Nhomincas, spécialistes de la pêche, et les Diolas-Djilas, originaires du sud du Sénégal et peuple marchand. ${ }^{2}$ Cette liste, loin d'être exhaustive, doit nous permettre de comprendre qu'outre l'aspect proprement culturel de la chose, le respect et l'exploitation

\footnotetext{
1 நbidem.

2 Notons que les documents suivants traitent, à des degrés divers, des spécialisations ethniques: Diana Lima Handem, Nature et fonctionnement du pouvoir chez les Balanta Brassa. Bissau, IN.E.P., 1986, 271 pages;

Anne-Marie Hochet, Seco Uldely, Les ex-royaumes Pepel du Tor et du Biombo: zones d'émigration temporaire: situation socio-économique Guinée-Bissau, Commissariat à la Coordination Economique et au Plan, novembre 1980, 46 pages;

Ministère de la Coordination Economique, du Plan et de la Coopération Internationale, op. cit., 37 pages; et, S.U.C.O.-C.R.A.D., Volumes I et II, op.cit.
} 
en vue du développement, des spécialités de chaque ethnie, permettront de maximiser l'effet, chacune transformant ses spécialisations en une certaine forme d'art maîtrisé d'elle seule. Par exemple, est-il courant de voir des hommes pepels aller de villages en villages pour couper et récolter les régimes de palmistes en échange d'argent ou de vivres. ${ }^{1}$ Cette forme de cooperation inter-ethnique est tout de même assez répandue en Guinée-Bissau, n'avons-nous à souligner une seconde fois, par exemple, la transmission par les Balantes, de leur technique de riziculture en eau salée à d'autres ethnies. Par contre, certaines associations inter-ethniques ne sont pas possibles ou ne le sont que difficilement. Ainsi en est-il des associations entre les Foulas et les Mandingues avec les Balantes. D'un autre côté, les Foulas et les Mandingues font bon ménage avec les Bijagos qui, pour leur part, s'entendent avec les Nhomincas et les Mancagnes, ceux-ci pouvant très bien coopérer avec les Balantes. ${ }^{2}$

\subsubsection{Division sexuelle du travail}

Aux spécialisations ethniques, il convient d'ajouter celles attribuées aux sexes. Nous avions déjà remarqué une spécialisation des sexes dans des activités spécifiques lors de l'étude du maraîchage et du Tableau 3.3 notamment. Le Tableau 3.6 dresse un portrait de la répartition sexuelle des principales activités du calendrier agricole de la région, ce qui nous permet de constater que tous les membres de l'unité familiale d'exploitation, hommes, femmes et enfants, participent à la production agricole.

1 Anne-Marie Hochet, Paysanneries en attente: Guinée-Bissau, op. cit., page 86.

2 Entrevue avec Paul Rouillard, chef de projet du Projet de Développement Agricole Bolama-Bijagos du C.E.C.I., Bolama, 2 juin 1988. 
TABLEAU 3.6

Répartition des principales tâches agricoles selon l'âge et le sexe

Ile de Bolama (1)

\begin{tabular}{|c|c|}
\hline Aether & Particlpante \\
\hline \multicolumn{2}{|l|}{ Ris arbonis } \\
\hline 1. Ambaremere & $\mathbf{H}+\mathbf{B}+\mathbf{B}$ \\
\hline 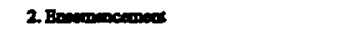 & $\mathrm{H}+\mathrm{P}, \mathrm{B}$ \\
\hline 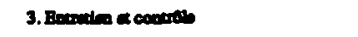 & $F+B$ \\
\hline 4. 2 resine & $F+B . B$ \\
\hline S. Tr.pose & $\mathbf{H}$ \\
\hline 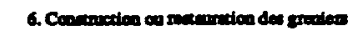 & $\mathbf{P}+\mathbf{H}$ \\
\hline \multicolumn{2}{|l|}{ 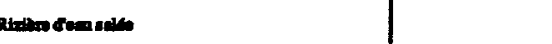 } \\
\hline 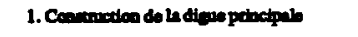 & $H$ \\
\hline 2. Deniche de la menowe & $\mathbf{H}$ \\
\hline 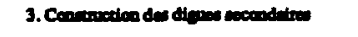 & st \\
\hline 4. Bneconescomon: & $\mathrm{H}+\mathrm{F}, \mathrm{B}$ \\
\hline S. Ropiquep & $\mathrm{H}+\mathrm{F}, \mathrm{B}$ \\
\hline 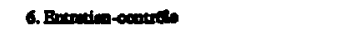 & $P+B$ \\
\hline 7. Rcoolen & $F+F+B$ \\
\hline \multicolumn{2}{|l|}{ 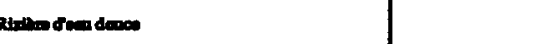 } \\
\hline 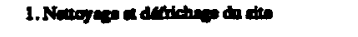 & $\mathbf{H}, \mathbf{F}$ \\
\hline 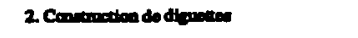 & $F$ \\
\hline 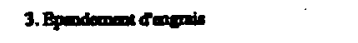 & $\mathrm{H}+\mathrm{F}$ \\
\hline 4.Lobous & Hi, polol P \\
\hline 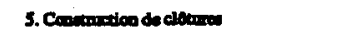 & H \\
\hline 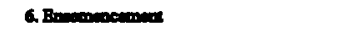 & $\mathbf{p}$ \\
\hline 7. Regranep & $\mathrm{H}+\mathrm{F}$ \\
\hline 2. Reowese & $\mathrm{H}+\mathrm{P}+\mathrm{B}$ \\
\hline Lamo & \\
\hline 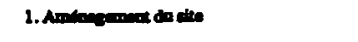 & $\mathbf{H}$ \\
\hline 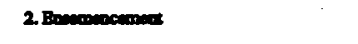 & $\mathbf{P}, \mathrm{H}$ \\
\hline 3.2100 ine & P.H \\
\hline 4.3enepe & $\mathbf{F}, \mathbf{H}$ \\
\hline S. Burepep & F,H \\
\hline
\end{tabular}

(1): H: hommes; F: femmes; E: enfants. Le signe + signifie que les participants travaillent ensemble; la virgule (,) précédant une lettre, indique la participation circonstancielle du sujet suivant la virgule.

Source: BOIES, Brigitte, ROUSSEAU, Alain, Guide pour l'analyse des systèmes de production; archipel Bolama-Bijagos, Guinée-Bissau, Projet de Développement Agricole Bolama-Bijagos, Bolama, C.E.C.I., mai 1988, Annexe. 
La contribution de la femme ne se limite cependant pas à l'agriculture et aux tâches familiales et domestiques d'ailleurs fort nombreuses telles la préparation des repas, le ramassage du bois de cuisine et de l'eau, la lessive, la fabrication de l'huile de palme, du savon, du sel, le séchage du poisson, la garde des enfants, etc.. En effet, elles pratiquent en plus toute une gamme d'activités, certaines entraînant des revenus monétaires faisant régulièrement d'elles la seule source d'entrée de revenu monétaire dans l'U.F.E., lorsque le mari ou d'autres membres de l'unité ne travaillent pas à l'extérieur. De fait, comme le souligne Anne-Marie Hochet:

"De même que l'on oublie que les femmes, elles aussi cultivent, de même on ne voit pas ou feint de ne pas voir que si les cultures étaient la seule activité économique des paysans et paysannes, la survie des populations rurales ne serait pas garantie.

Contrairement aux usages également, et à dessein, nous commencerons par l'analyse des activités économiques féminines dont les incidences sur le niveau de vie et la qualité de la vie sont extrêmement importantes, et qui ont l'avantage de présenter plus de souplesse et de variété que les activités masculines. Nous avons pu observer, en Guinée-Bissau comme dans d'autres pays de l'Afrique de l'Ouest, que, en dehors des cultures, les femmes pratiquaient des activités à revenus monétaires beaucoup plus longtemps que les hommes; certaines activités économiques sont même un peu considérées comme la spécialité des "vieilles" (fabrication de sel, teinture par exemple). Enfin, nous avons pu constater que dans certaines régions particulièrement déshéritées (ilha de Bolama, par exemple) les activités économiques féminines étaient le seul facteur permettant de freiner l'exode et d'éviter le dépeuplement.

Il nous faut encore considérer dans de nombreux cas, que la paysanne ici aussi se comporte comme un agent économique complet: elle produit, transforme, transporte et vend." 1

Les femmes ont effectivement une importance économique majeure à Bolama dans le soutien familial. Un recensement de la ville de Bolama et de ses villages et quartiers périphériques en 1988, avait constaté que près de $40 \%$ des habitations de Bolama (125 sur 327) étaient gérées et tenues par des femmes. ${ }^{2}$ Ce rôle prépondérant de la femme dans les

1 Anne-Marie Hochet, op.cit., page 113.

2 Entrevue avec Tina Stegemann, anthropologue, Ile de Paix, Bolama, 1 juin 1988. 
activités génératrices de revenus peut s'observer même dans les ethnies où les femmes jouissent le moins de liberte, ${ }^{1}$ les ethnies islamisées en l'occurrence. Chez les ethnies animistes comme les Balantes, la femme peut, en théorie, profiter de ses revenus comme elle l'entend, puisque sa qualité de procréatrice et de productrice, non reconnue au niveau politique, mais socialement admise, lui permet de jouir d'une grande liberté dans de nombreux domaines dont celui de l'économique et de la sexualité. Cette grande latitude au niveau des responsabilités économiques de la femme possède sa contrepartie chez l'homme qui détient la responsabilité et l'autorité pour subvenir aux besoins de la famille. Ainsi, tout ce que celui-ci obtient, produit ou achète doit être conservé en totalité dans le cellier familial sauf en cas d'abondance, ce qui n'est pas le cas pour la femme qui n'est, à ce point, astreinte à aucune obligation définie par la société balante. ${ }^{2}$ Dans la réalité par contre, la situation nous est apparue tout autre, et la femme s'avère bien souvent le principal soutien familial en dehors de la rizière:

"La libre disposition de son argent confirme la femme dans son rôle économique et social et la valorise; cependant le fait d'en avoir la libre disposition ne signifie pas, pour la paysanne, qu'elle utilise, dans la pratique, cet argent à son profit personnel. Nous avons constaté que les femmes ne dépensent pas leur argent seulement pour s'acheter des habits, des feuilles de tabac ou quelques objets de confort, mais qu'elles le dépensent aussi à l'achat de céréales quand l'homme ne peut plus le faire, à l'habillement des enfants, à l'amélioration des sauces. Nous voyons beaucoup de femmes acheter pour les hommes du vin de palme et du tabac, "gagner leur paradis" selon une forme de pensée Mandingue et musulmane." 3

\footnotetext{
1 Anne-Marie Hochet, op. cit., page 113.

2 Diana Lima Handem, op.cit., pages 71-72.

3 Ibid, pages 113-114.
} 
La division sexuelle type des activités de production pouvant dégager des revenus s'établit comme suit: ${ }^{1}$

-Activités féminines:

- maraîchage

- cueillette

- petit élevage

- activités de transformation: vin de cajou, huile de palme, savon, sel, etc.

- fabrication d'objets artisanaux: vannerie et poterie

- pêche et cueillette de petits poissons, crustacés et mollusques

- commerce de produits récoltés ou de plats cuisinés.

-Activités masculines:

- cultures de rente: riz, arachide, maïs, mil, sorgho

- élevage

- pêche et chasse

- tissage

- cueillette des fruits du palmier et du vin de palme

- forge, couture, vannerie

- boulangerie

- petit commerce temporaire ou occasionnel.

${ }^{1}$ Ibid. pages 114-141. 
3.4.3 Repartition saisonnière des activités

D'une manière générale, et pour permettre une certaine récapitulation de ce que l'on pourrait qualifier d'activités traditionnelles (agriculture, cueillette, pêche et artisanat), nous avons divisé l'ẹsemble des activités selon la saison où elles sont exécutées: ${ }^{1}$

-Activités de saison des pluies:

- culture:

- riz

- mil

- maiis

- arachide

- patate douce

- manioc

- pois bijago

(pois bambara)

- haricot

- autres cultures secondaires:

- melon

- citrouille

- concombre

- etc.

- pêche

1 Brigitte Boies, Alain Rousseau, Guide pour l'analyse des systèmes de production: archipel Bolama-Bijagos. Guinée-Bissau, op.cit., pages 8-9. 
- exploitation du palmier

- cueillette

- élevage.

-Activités de saison sèche:

- maraîchage

- pêche et chasse (lorsqu'il y a lieu)

- préparation des cultures de saison des pluies

- entreposage des récoltes

- cueillette

- exploitation du palmier

- cueillette et transformation du cajou

- construction et autres travaux (puits, tissage, construction de cases, réparation des toits de case, réparation des digues)

- commerce

- élevage

- cérémonies.

Parmi les activités de la saison sèche, nous avons inclus les cérémonies bien, qu'en soi, à nos yeux d'occidentaux du moins, celles-ci ne relèvent pas des activités productrices. Elles en font pourtant partie puisqu'elles s'intègrent dans la démarche normale de tout processus poursuivi par la population locale, qu'il soit d'ordre productif (agricole ou autre) ou strictement religieux. De plus avons-nous jugé pertinent de les y inscrire vu leur importance tant en matière de temps que de ressources engagées (consommation des réserves de 
nourriture). Les ethnies bolamiennes, tout comme celles des autres régions du pays, qu'elles soient animistes, musulmanes ou même catholiques, pratiquent toutes, à des degrés divers, des séances d'appel à la clémence des divinités, surnommées "Iran", qui se trouvent partout et animent chaque chose, la rizière, la mer, la maladie, etc.. Ces séances, appelées cérémonies, qui sont d'ailleurs fort nombreuses, s'adaptent à toutes les occasions (initiation, maladie, mort, préparation des cultures, récoltes, guérison, etc.) et peuvent durer de longues journées, parfois même des semaines comme le démontre le suivi les maraîchères d'un village mancagne, dont les activités ont été perturbées pendant près d'un mois lors de la mort de deux de ses habitants. 1

\subsection{Santé et éducation}

\subsubsection{Santé}

Les conditions d'hygiène déplorables que l'on rencontre dans la région Bolama-Bijagos sont directement responsables d'une situation sanitaire précaire où la mortalité infantile atteint les 200 pour mille chez les enfants de moins d'un an et les 45 pour mille pour les enfants âgés de un à cinq ans. ${ }^{2}$ Habitations en terre dont le sol est infesté de puces et de tiques, absence de toilettes décentes, sources d'eau infestées, et présence de moustiques anophèles, comptent parmi les causes les plus évidentes à la base de ce triste bilan. Une situation semblable existe à Bolama, et on peut en déduire que le taux de mortalité infantile y est aussi élevé que dans l'ensemble de la région, même si aucune étude n'en fait explicitement état.

1 Brigitte Boies, Alain Rousseau, Enquête sur la commercialisation des produits maraîchers, op. cit., pages 32-33.

2 Anne Lovell, République de Guinée-Bissau formulation du projet GBS/85/CO1. Infrastructures de santé dans les îles Bijagos, troisième partie, s.l., Fonds d'Equipement des Nations-Unies (F.E.N.U.), Bureau pour l'Exécution des Projets (B.E.P.), août 1985, page 1. 
Le régime alimentaire bolamien contribue, par un déséquilibre alimentaire certain, à l'apparition, surtout chez les enfants, de nombreux cas de malnutrition légère, ce qui rend le sujet plus sensible aux affections courantes (rougeole, pneumonie, paludisme, etc.) ${ }^{1}$ et, chez les femmes enceintes, à celle de nombreux cas d'anémie. En effet, comme nous l'avons précédemment noté, celui-ci est basé sur la consommation quasi-exclusive de riz, occasionnellement additionné d'huile de palme, de mollusques ou de poisson. La consommation de légumes et de viande est plutôt rare hormis celle de quelques légumes traditionnels (manioc, patate douce, gombo, etc.). L'huile de palme pour sa part, voit sa consommation être moins courante à Bolama que chez les populations des autres îles de l'archipel où l'abondance de cette denrée garantit une utilisation plus fréquente.

A Bolama, on compte un hôpital et un centre de santé situé à Uato Fula. L'hôpital de Bolama est d'envergure régionale. Sa construction remonte aux années $1882-1883 .^{2} \mathrm{Il}$ dispose de 76 lits, ${ }^{3}$ et est sous la supervision de deux médecins coopérants cubains venus remplacer, en 1988, l'équipe de Guinée-Conakry en poste depuis plusieurs années. Le centre de santé de Uato Fula s'avère être en fait plutôt un poste sanitaire n'ayant comme tout effectif qu'un infirmier auxiliaire et un employé d'entretien. ${ }^{4}$

L'hôpital de Bolama affiche un aspect négligé et on y note un manque flagrant de matériel. Il ne constitue pas un cas à part car tout le réseau de santé de la région présente le même portrait, comme en font foi Côté et Demers:

1 Marthe Côté, André Demers, Repères pour un développement rural intégré dans la région de Bolama. Guinée-Bissau, op. cit., page 161.

2 Gabinete do Ministro residente, Brojecto de Câmara Municipal. Bolama, Provincia do Sul, março 1988, Bolama, page 10.

3 D.P.R.-Bolama-Bijagos, Diagnostico da regiăo de Bolama-Biiagos. Bolama, 23 de agosto de 1986, page 10.

4 Anne Lovell, op.cit, pages 37-57. 
"L'infrastructure sanitaire de la région s'avère nettement insuffisante. La plupart des postes sanitaires manquent de l'équipement de base le plus élémentaire et l'état des lieux laisse franchement à désirer. Dans l'ensemble de l'archipel, un seul des postes sanitaires - celui de Canhabaque - dispose d'un réfrigérateur à pétrole pour conserver sérums et vaccins. Si les communications sont très difficiles de Bissau à l'archipel et d'une île à l'autre, elles le sont également à l'intérieur même des îles. Dans le cas où une île ne dispose que d'un seul poste sanitaire, par exemple, les malades doivent parfois marcher de longues heures sur des sentiers de brousse pour atteindre le poste; d'où la nécessité de faciliter l'accès des postes sanitaires aux malades, et, inversement aux infirmiers l'accès des "tabancas".

Quant aux médicaments qui, jusqu'à maintenant, proviennent tous de l'étranger, ils ne parviennent dans la région qu'à tous les trois mois; toutefois, d'après le médecin de Bubaque, on les reçoit en quantités qui devraient être suffisantes si on les utilisait de façon rationnelle, ce qui n'est pas toujours le cas. Ceci met en cause un problème, celui de la formation très inégale des responsables des postes sanitaires, où l'on trouve tantôt d'excellents infirmiers ou secouristes, tantôt des gens qui font preuve davantage de bonne volonté que de compétence." 1

D'après les registres de l'hôpital de Bolama, les cas les plus fréquents de consultation médicale sont dans l'ordre: le paludisme (dont la forme la plus courante est le paludisme à p. falcitarum), les parasitoses, les dermatoses, les diarrhées, l'anémie, les blessures et traumatismes des membres, et enfin les blennorragies, sans oublier, chez les enfants, les maladies infectieuses (rougeole et varicelle). De plus note-t-on la présence de l'infestation par le filaire de Bancroft (W. Bancrofti) et de l'éléphantiasis. ${ }^{2}$ Du côté des femmes enceintes, on estime à $3 \%$ celles qui meurent à l'accouchement ou de ses suites. ${ }^{3}$

Il est intéressant de remarquer, et pour corroborer nos constatations antérieures selon lesquelles lîle de Bolama est beaucoup plus liée avec la partie sud du pays qu'avec le reste de l'archipel, que l'hôpital de Bolama traite en majorité les gens de l'île de Bolama et de la région

\footnotetext{
1 Marthe Côté, André Demers, op. cit., pages 166-167.

2 Ibid., page 161.

3 Anne Lovell, op.cit., page 33.
} 
de Buba située sur le continent, alors que la population de Bubaque et de l'archipel se dirige surtout vers l'hôpital de Bubaque. ${ }^{1}$

\subsubsection{Education}

Au niveau de l'éducation, malgré l'obligation pour les enfants de fréquenter les quatre premières années d'école primaire, le taux d'analphabétisme demeure très élevé dans le pays: $90,4 \%$ de la population totale du pays ${ }^{2}$ et à Bolama, $89 \% .^{3}$

Le système d'éducation bissau-guinéen se présente ainsi: ${ }^{4}$ -Enseignement Elementaire de Base (E.B.E.): $\quad$ première, deuxième, troisième et quatrième années;

-Enseignement Complémentaire de Base (E.B.C.): cinquième et sixième années; -Enseignement Secondaire Général (E.S.G.): $\quad$ septième, huitième et neuvième années; -Enseignement Secondaire Complémentaire (E.S.C.): dixième et onzième années.

Les statistiques en matière d'éducation sont disponibles seulement pour la région Bolama-Bijagos et non pour l'île. Elles indiquent une situation alarmante qui ne peut que mener à une remise en question de cet enseignement toujours donné en portugais alors que plus de la moitié de la population du pays parle créole. ${ }^{5}$ On estime que le taux de fréquentation scolaire de la population en âge d'aller à l'école dans la région est inférieur à

1 Marthe Côté, André Demers, op. cit., page 161.

2 Guinée-Bissau, Conférence des Organisations Non Gouvernementales: document d'orientation, Bissau, 7-10 novembre 1985, page 11.

3 F.E.N.U., Construction d'infrastructures scolaires et mise en place d'un nouveau programme d'enseignement en milieu rural dans les îles Bijagos: accord de projet, s.1., GBS/85/CO2, 1985, page 6.

4 Ibid., page 5.

5 Guinée-Bissau, op cit. page 3. 
50\%. ${ }^{1}$ Le taux d'échec, c'est-à-dire le taux d'élèves redoublant leur année, est de $52 \%$ et le taux d'abandon frise les $40 \% .^{2} \mathrm{La}$ fréquentation scolaire diminue elle aussi au fur et à mesure de la progression en grade: ainsi, en 1978-1979, les écoliers en première année étaient au nombre de 558, passant à 381 en deuxième année, 234 en troisième année et 237 en quatrième année. ${ }^{3}$ Cette tendance à l'abandon est encore perceptible car, pour l'année scolaire 1987-1988, le nombre des écoliers du secteur de Bolama s'élevait à 558 en première année, pour diminuer à 502 en deuxième année, 342 en troisième année et 277 en quatrième année. ${ }^{4}$

Côté et Demers avaient remarqué la relative faiblesse de la représentation féminine au niveau de l'enseignement de base élémentaire, calculant le nombre de filles à moins de 50\% des élèves. ${ }^{5}$ Nous avons aussi constaté ce fait pour le secteur de Bolama où les filles ne représentent guère que $47,1 \%$ des écoliers en $1987-1988,{ }^{6}$ alors que la proportion de filles des catégories d'âge de cinq à neuf ans et de dix à 14 ans, par rapport aux garçons du même âge s'établit à 51,8\%.7 Ces chiffres tendent à confirmer le fait que les bolamiens accordent moins d'importance à l'éducation scolaire des filles qu'à celle des garçons, considérant probablement comme superflue l'instruction des filles dont la présence à la maison est nécessaire à la réalisation de certains travaux domestiques.

La ville de Bolama compte quatre écoles élémentaires de base, une école d'enseignement complémentaire de base, un lycée, une école normale, une école de formation de professeurs, une école pilote du Parti et un jardin d'enfants. Ainsi s'explique facilement la

\footnotetext{
1 Marthe Côté, André Demers, op. cit., page 174.

2 F.E.N.U., op. cit., page 6.

3 Marthe Côté, André Demers, op.cit., page 174.

4 Compilation effectuée à partir des données de la Délégation Régionale de l'Education, Bolama, 1988.

5 Marthe Côté, André Demers, op.cit, page 174.

6 Délégation Régionale de l'Education, op. cit.

7 Comité de Estado do Sector de Bolama, Recenseamento de 1987, Bolama, 1987, compilation interne.
} 
raison de l'octroi à la ville de Bolama, du titre de capitale culturelle de la Guinée-Bissau, vu notamment l'importante quantité d'écoles qu'elle abrite par rapport à son importance démographique. Huit autres petites écoles d'enseignement élémentaire se répartissent sur l'île ( Caboupa, Casseque, Cassucaï, Ga-Moria, Lala, Madina, Pujunguto et Uato Fula ) ${ }^{1}$ et fonctionnent durant les années où elles peuvent disposer d'un professeur.

La précarité de la situation de l'enseignement à Bolama repose sur plusieurs facteurs dont le manque de professeurs et, faut-il le constater, une certaine faiblesse académique de leur part, puisqu'ils n'ont en général qu'une quatrième année et n'ont suivi aucun cours de pédagogie. De plus, ces professeurs arrivent au village, dans le cas des écoles de village, à la fin de la saison des pluies, donc trop tard pour semer leur riz et ainsi,

..."reçoivent tant bien que mal leur subsistance d'une population locale qui les considère toujours comme des étrangers. Ils ne demeurent habituellement pas plus de trois ans et logent dans une maison qu'ils construisent eux-mêmes dans le village." 2

La réticence qu'ont les populations locales face à l'éducation scolaire est facilement perceptible et trouve ses racines au sein de phénomènes ethno-culturels plus marqués en campagne qu'en ville, les ruraux étant eux-mêmes déjà moins scolarisés que les habitants de la ville. Côté et Demers font la démonstration de ces réticences face à la scolarisation chez les Bijagos. Cette démonstration peut, à notre avis, s'appliquer dans ses grandes lignes aux autres ethnies de Bolama. Ils nous expliquent comment les valeurs traditionnelles bijagos participent, au moyen des rites initiatiques et du travail quotidien du jeune, enfant et jeune adulte, à l'éducation de celui-ci et à la reproduction de la société. En outre, l'apport économique que fournit le jeune à la survie de l'ensemble de la collectivité et, en l'occurrence, à celle des vieux, pose une contrainte de taille à la scolarisation qui, en plus de

1 D.P.R.-Bolama-Bijagos, Inquérite de tabanca, op. cit.

2 F.E.N.U., op cit. page 6 
véhiculer un ensemble de valeurs nouvelles que ne partage pas la société traditionnelle, menace la sécurité matérielle de ses membres les plus âgés puisque les jeunes travaillent tant pour leur propre bien-être et celui de leur famille, que pour celui des Anciens. Tout cela, ajouté au fait que le calendrier scolaire n'est aucunement compatible avec le calendrier agricole et les périodes de cérémonies et d'initiation, ne peut que provoquer un sentiment de rejet de la part des autorités ethniques traditionnelles (les Anciens) qui craignent, non sans raison d'ailleurs, de voir les éléments les plus dynamiques du village, les quitter pour aller chercher ailleurs ce qui peut leur paraître comme de meilleures conditions d'existence. ${ }^{1}$

Enfin, l'inefficacité du système scolaire puise sa source non seulement dans les problèmes logistiques ou dans les résistances culturelles qu'il rencontre, mais aussi dans le fait que les programmes sont issus des modèles européens et qu'ils répondent davantage aux besoins des milieux urbains qu'à ceux du monde rural. ${ }^{2}$ Sans changement notoire, ce système ne peut qu'être condamné à continuer à former des fonctionnaires plutôt que des agriculteurs ou des travailleurs productifs et utiles à leur collectivité.

\subsection{Commerce et troc}

La présence sur lîle de Bolama de trois modes de production différents se répercute, au niveau des échanges, par une situation où deux types d'échanges s'effectuent: le troc et la vente. On sait que la marchandise comporte une valeur d'usage et une valeur d'échange où la première fait référence à l'utilité que les acteurs économiques pourront en tirer, alors que la seconde, plutôt que de porter sur l'aspect qualitatif, établit un rapport quantitatif dans lequel

\footnotetext{
1 Marthe Côté, André Demers, op.cit., pages 169-170.

2 F.E.N.U., op cit., page 7.
} 
des marchandises à valeurs d'usage différentes s'échangent l'une contre l'autre via l'intervention d'un étalon, la monnaie. ${ }^{1}$ Ainsi,

"Toute marchandise est destinée à la consommation. Mais celui qui produit la marchandise ne se confond pas avec celui qui la consomme; d'où la nécessité de l'échange.

Dans les économies naturelles, les échanges s'effectuent sous forme de troc, marchandise contre marchandise; ce qui suppose des contacts personnalisés entre les différents producteurs et ce qui aussi limite considérablement le volume des échanges.

Dans les économies modernes et marchandes, les échanges sont réalisés par l'intermédiaire de la monnaie, qui se présente comme l'équivalent général pour toutes les marchandises." 2

L'économie de troc était la base naturelle des échanges de la population de l'île. Avec l'arrivée de la colonisation portugaise et, d'une manière générale, de l'ouverture ou plutôt de la pénétration du pays par le mode de production capitaliste, nous avons pu voir peu à peu apparaître le salariat et une certaine économie marchande basée sur la valeur d'échange. L'influence du mode de production capitaliste ne se fait pas sentir uniquement en ville, le monde rural en est peu à peu affecté. Ainsi, les communautés rurales, bien établies dans leur mode de vie traditionnel d'autoconsommation, s'ouvrent progressivement à l'économie marchande, surtout pour satisfaire de nouveaux besoins de consommation que ce soit pour se vêtir avec des tissus manufacturés ou fumer du tabac par exemple.

Les principaux changements à ce titre depuis la colonisation concernent l'imposition obligatoire de tout adulte de plus de 18 ans et la monétarisation des activités du travail. ${ }^{3}$ Dès 1913, d'ailleurs, un texte de la Sous-Commission du Conseil d'Outre-Mer relevait l'importance de l'imposition comme instrument de passage à l'économie monétaire:

1 Makhtar Diouf, Economie Politique, Dakar, Nouvelles Editions Africaines, 1981, Tome 2: Théorie économique nécclassique et marxiste, page 196.

2 Ibid., page 198.

3 S.U.C.O.-C.R.A.D., Volume II, op.cit., pages 57-58. 
"Les recettes de la province devraient être soutenues par la fiscalité directe, impôt de paillote et autres, plus que par tout autre moyen. Ces impôts sont en même temps une forme indirecte d'obliger les indigènes à produire, une démonstration irréfutable de l'exercice d'une occupation effective." 1

De nos jours, toute personne adulte, ne fréquentant pas l'école, qu'elle vive en ville ou en campagne, qu'elle soit salariée ou non, doit, une fois l'an, disposer d'une certaine somme d'argent pour régler sa dette à l'Etat . En 1987, l'impôt était ainsi de 5000 P.G. pour les hommes et de 2500 P.G. pour les femmes. Ceux qui ne disposent d'aucune source de revenu devront se résoudre à vendre un quelconque produit ou encore à offrir un service contre rémunération.

Traditionnellement, chez les différentes ethnies, nous avons pu constater que les relations économiques et les relations sociales forment un tout. Dans ce cadre, les corvées collectives comme la préparation d'une rizière ou la construction d'une case, qui sont fondées sur des obligations statutaires telles que le devoir des jeunes envers les aînés, par exemple, ou encore sur la simple collaboration et solidarité ethnique, remplacent ce que d'autres sociétés effectueraient sur la base du salariat ou du travail forcé. Le travail se fait donc, dans ce cas, collectivement, et en échange de nourriture, de boisson ou de services divers. Ce mode de fonctionnement est encore en vigueur à Bolama. Cependant, il semble en perte de vitesse, notamment dans le cadre d'activités dont la finalité est le dégagement de revenus monétaires ainsi que l'atteste l'embauche par les maraîchères de personnes pour effectuer certains travaux dont le creusage de puits et l'émondage des palmiers. ${ }^{2}$ Il en est de même dans le secteur de la

\footnotetext{
1 José Capela, 0 imposto de palhota e a introducăo do modo de producão capitalista nas colónias, page 7, cité dans, Carlos Lopes, "Ethnie, Etat et rapports de pouvoir en Guinée-Bissau", Itinéraires, notes et travaux, No. 22, mars 1982, Première Edition, Genève, page 48.

2 Brigitte Boies, Alain Rousseau, Enquête sur la commercialisation des produits maraîchers, op. cit, pages 67-69.
} 
pêche où les Nhomincas engagent des pêcheurs locaux pour travailler à leur service, ${ }^{1}$ ou encore dans différents secteurs d'activité en ville, où le salariat est de rigueur.

Au niveau des échanges, on comprendra que le troc aura encore sa place et visera à compenser le déficit vivrier d'un paysan ou encore jouera le rôle de substitution de production dans le cas des ethnies qui migrent (Pepels par exemple) ${ }^{2}$ ou qui se spécialisent dans certaines activités ne leur permettant pas de toujours disposer des produits nécessaires (comme les Nhomincas qui pêchent).

Les courantes pénuries de toutes sortes entraînent la population locale vers les échanges en nature plutôt que ceux basés sur la monnaie, cette dernière ne leur offrant aucune garantie d'obtenir le produit désiré. ${ }^{3}$ Il est aisé de comprendre, dans ce cadre, la persistance du troc et l'inévitable marginalisation de la monnaie. En ce sens, la mentalité des populations rurales est demeurée très conforme à la culture traditionnelle et la valeur d'usage prime largement sur la valeur d'échange. La conséquence la plus palpable en est sûrement l'absence quasi-générale de liens commerciaux entre la ville et la campagne qui, de ce fait, ne joue pas le rôle de grenier alimentaire qu'on pourrait attendre d'elle. Le faible effet incitatif de la monnaie sur la population se traduit aussi au niveau de l'Etat qui, comme nous l'avons déjà vu, n'hésite pas, afin de dynamiser la récolte de cajou, à instituer l'échange de sa noix contre du riz. De manière plus générale, quiconque veut obtenir, contre argent, un produit de la terre ou un animal, rencontrera de nombreuses difficultés qui n'auront d'équivalent que le nombre de tractations et de rencontres nécessaires à la réalisation de ses objectifs, et la nécessité d'y investir des sommes d'argent pouvant paraître, aux cours officiels du peso, relativement élevées.

\footnotetext{
1 Ibid., pages 47-48.

2 Ibid, pages 71-72.

3 Ibid. pages 95-96.
} 
Il faudra donc retenir que le monde rural bolamien, bien que basant encore ses échanges selon des méthodes traditionnelles, n'est tout de même pas complètement étranger aux échanges marchands, comme le souligne Carlos Lopes:

"Bien que le mode de production capitaliste soit actuellement prépondérant du fait de l'intégration aux structures de marché, au niveau interne et externe, il subsiste toute une organisation économique qui échappe à la logique de la société marchande. Dans le milieu rural, les ethnies dont la composante sociale majeure est paysanne, vivent en autarcie sans pour autant être isolées de l'économie monétaire." 1

L'approvisionnement nettement déficient à Bolama comme ailleurs dans le pays, trouve sa source, pour une bonne part, dans la structure même du commerce. Après l'indépendance, les deux sociétés étatiques commerciales, Socomin pour les biens d'équipement et les Armazens do Povo pour les biens de consommation, se sont vues, comme nous l'avons précédemment remarqué, attribuer le monopole de l'exportation des produits prioritaires (cajou, noix de coco et de palmiste, arachide, etc.) et de l'importation. Il en résulta l'obligation, pour les marchands privés, d'effectuer leurs achats uniquement chez ces deux grossistes nationaux. Le commerce privé, se voyant ainsi interdire le rôle de fournisseur de denrées alimentaires essentielles telles que le riz, l'huile, le sucre, etc., fut relégué peu à peu à celui de fournisseur de produits jugés non-stratégiques: allumettes, fils, tissus, chaussures, produits de toilette, etc. ${ }^{2}$. Qui plus est, les vices propres aux déficiences en matière de transport et à un manque flagrant de productivité des employés-fonctionnaires, se sont concrétisés dans la généralisation des étals vides chez les marchands privés et étatiques. De nombreuses visites et contacts avec les Armazens do Povo de Bolama, ont d'ailleurs permis de constater la présence de réseaux souterrains d'approvisionnement en différents produits pour les membres de l'élite locale. De même, le manque d'esprit d'initiative des employés et

\footnotetext{
1 Carlos Lopes, op_cit., page 90.

2 Ibidem.
} 
des cadres de ces commerces, fait que de nombreux produits traînent des mois et même des années sans jamais être mis en vente, sous prétexte que les listes de prix ne sont pas disponibles, ce qui peut, en soi, constituer un excellent moyen d'entreposer des produits destinés à une distribution restreinte. Anne-Marie Hochet résume très bien le dilemme qui affecte la population rurale de Bolama en ce qui a trait à son intégration à l'économie monétaire marchande:

"Les dépenses courantes demeurent très réduites, non pas en vertu d'une auto-consommation et d'une autarcie économique coutumière (comme le véhicule un certain mythe de la société Bijagos) mais surtout faute d'approvisionnement et en raison de la régression économique qui touche l'île. Les Bijagos de Bolama sont depuis longtemps dans le système monétaire; y sont-ils entrés à leur avantage? On doit ici tenir compte des réflexions qu'ils émettent eux-même sur leur propre sort: "on nous a conseillé de vivre autrement, de nous habiller de tissus, et de pagnes (au lieu des saya [jupes de paille] de nos femmes); maintenant nous n'arrivons plus à acheter de vêtements et les vêtements que nous avons se déchirent quand on monte au palmier, alors que les peaux étaient beaucoup plus solides..." Humour, ironie?. Réalité de la frustration aussi: "on ne peut plus continuer à être civilisés" entend-on dire par des hommes dont on a condamné jadis la personnalité et la civilisation propre"... 1

Face au manque de dynamisme du commerce étatique, le gouvernement a entrepris, à partir de 1982, une vaste réforme commerciale limitant progressivement le rôle des deux sociétés d'Etat à celui de grossistes et d'agents d'import-export et remettant peu à peu au domaine privé le commerce de détail. ${ }^{2}$ Le Premier Plan Quadriennal de Développement Economique et Social (1983-1986) en fait état, précisant l'élargissement du rôle du secteur commercial privé de détail, où les deux entreprises d'Etat conserveront le monopole des importations et de la distribution en gros des articles vitaux, dont les produits de l'aide

1 Anne-Marie Hochet, La situation économique en milieu rural dans le secteur de Bolama. Note de travail. Bissau, le 22-2-81, page 7.

2 Philippe de Braconier, Elizabeth Drory, Rapport de mission de juin 1985: prétude de l'Ile de Paix de Bolama, op. cit., page 4. 
alimentaire, et le monopole des trois produits dits traditionnels: arachide, noix de coco et de cajou. 1

Le gouvernement adopta progressivement un "Programme de Stabilisation Economique et Financière" qui lui fit reconnaître, en août 1986, la nécessité d'une restructuration profonde du commerce en stimulant l'action du commerce privé au moyen d'une majoration de sa participation dans tous les domaines de la commercialisation des produits dont l'exportation (sauf pour les trois produits stratégiques) et l'importation (sauf pour les céréales, réservées aux Armazens do Povo). Le monopole que se réservait l'Etat sur l'obtention des devises étrangères est du même coup en quelque sorte aboli puisqu'on permet, à partir de 1986, aux commerçants privés d'importer jusqu'à 50\% de la valeur F.O.B. des marchandises qu'ils ont exportées. ${ }^{2}$ Le monopole d'Etat sur les devises avait déjà été assoupli dès le début $\mathrm{du}$ Premier Plan, mais la permission accordée aux marchands privés à ce moment d'obtenir jusqu'à l'équivalent de $70 \%$ de la valeur de leurs exportations en devises, n'était que très difficilement réalisable par la Banque Nationale, vu son propre manque de disponibilité de devises. 3

Le même programme reconnaît la détermination, par les agents économiques et les mécanismes de la circulation des produits, des prix, suivant laquelle les prix, dits "libres", oscillent entre un prix maximum et un prix minimum. 4

Les conséquences de cette politique ont commencé à se faire sentir à Bolama, surtout à partir de la fin de 1987 et du début de 1988. Les cinq commerces privés de Bolama ont, peu à peu, diversifié leurs marchandises et il devenait alors possible d'y trouver des produits aussi

1 MICEPCI, Plano Quadrienal de Desenvolvimento Económico e Social 1983/1986: Plano anual 1985, Republica da Guiné-Bissau, page 16.

2 Republica da Guiné-Bissau, Boletim Oficial, No. 32, quarta-feira, 13 de agosto de 1986, décret No 22/86.

3 Philippe de Braconier, Elizabeth Drory, op. cit., page 4.

${ }^{4}$ Republica da Guiné-Bissau, Boletim Oficial, No. 32, quarta-feira, 13 de agosto de 1986, décret No 23/86. 
variés que du sucre, de l'huile, du riz, des tomates en conserve, de la pâte de tomate en conserve, du lait en poudre ou en conserve, des pommes de terre, etc., tous des produits pratiquement introuvables auparavant.

L'envers de la médaille concerne cependant la forte hausse des prix survenue à cette même époque. Cette inflation, extrêmement difficile à supporter pour la population urbaine particulièrement, dont les salaires n'ont pas été ajustés en conséquence, est comparable, dans ce pays qui a beaucoup recours à l'importation pour satisfaire ses besoins de consommation, à la dévaluation subite du peso de $250 \%$ en mai 1987 . A titre d'exemple, un passage par bateau, de Bolama à Bissau, a augmenté de 253,85\% durant cette période, ${ }^{1}$ alors qu'il avait déjà été majoré de plus de 400\% comparativement à l'année précédente (1986: 150 P.G., début 1987: 650 P.G., mai 1987: 1650 P.G.). Les seules données précises concernant l'inflation s'appliquent à 1984 , où on évaluait celle-ci à $75 \%$, parallèlement à une dévaluation du peso de l'ordre de $289,4 \%$ pour la même époque (le 23 décembre 1983, un dollar américain valait 44 P.G. alors qu'un an plus tard, le 31 décembre 1984, il en valait 127,33). ${ }^{2}$

Outre ces deux commerces publics et les cinq boutiques privées de la ville de Bolama, nous retrouvons à l'autre extrémité de lî̀le, à Bolama de Baixo, un commerce privé tenu par un Foula. On y remarque d'ailleurs la présence d'une machine à décortiquer les noix de palmistes, dont les services sont, notons-le, très prisés de la population bijago de l'île de Gallinhas. Bolama ne compte pas d'autres commerces privés hormis deux petites boulangeries qui fonctionnent au rythme des arrivages de farine, et de trois "pensões", petits restaurants des plus modestes.

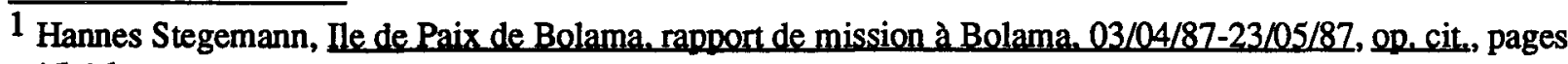
15-16.

2 Bernardino Cardoso, Mémorandum à l'intention de l'O.J.T. (Organisation Internationale du Travail): traduction résumée, cité dans, Philippe de Braconier, Elizabeth Drory, op. cit., Annexe. 
Cependant, le lieu central de toute l'activité commerciale, du moins en ce qui concerne l'aspect social de celle-ci, s'avère être le marché de Bolama, situé dans l'ancienne partie de la ville:

"L'attraction qu'exerce le marché n'est pas seulement économique, elle est aussi sociale. Nombreux sont les flâneurs qui viennent simplement aux nouvelles. La finalité du marché est double: il s'agit d'abord d'écouler la production à petite échelle et de se procurer des biens de consommation, mais aussi de se regrouper pour maintenir le contact entre les villages et les groupes sociaux ." 1

Le marché de Bolama est le principal endroit où se vendent légumes, poisson et, occasionnellement viande. A l'intérieur du marché, se trouvent huit petits kiosques où l'on peut se procurer toute une gamme d'articles (lampes de poche, piles, cola, pagnes, sandales, vêtements usagés, etc.). Au centre du marché sont alignées une quarantaine de tables de béton servant aux femmes à étaler leur marchandise composée uniquement de nourriture (laitue, tomates, oignons, bananes, noix de coco, pâte d'arachide, etc.) et de savon, selon la disponibilité et la saison. Le marché est ouvert tous les jours, et les vendeuses s'y rendent vers huit heures du matin. Règle générale, chaque vendeuse occupe toujours la même table, les meilleures places revenant aux revendeuses attitrées, celles qui achètent des produits qu'elles revendent, contrairement aux autres qui y écoulent leur propre production. ${ }^{2}$

Le marché est tenu par la comité d'Etat régional qui fixe une taxe aux commerçantes selon la nature et la quantité de leur produit. Cette taxe varie entre 150 et 400 P.G. pour une bassine de tomates, 50 et 100 P.G. pour quelques oignons... Les prix, hormis ceux de la viande et du poisson qui sont fixes bien que cela ait tendance à changer depuis la libéralisation du commerce, sont établis par les vendeuses elles-mêmes. On remarque une certaine entente

1 S.U.C.O.-C.R.A.D., Volume II, oD.cit., page 77.

2 Brigitte Boies, Alain Rousseau, Enquête sur la commercialisation des produits maraîchers, op. cit., pages 56. 
entre celles-ci et les prix sont uniformes, si ce n'est pour les produits rares ou occasionnels pour lesquels on ne constate pas toujours de consensus. Les plus forts taux de vente sont enregistrés entre huit heures et neuf heures trente du matin et, vers onze heures, il ne reste pratiquement plus de vendeuses sur place, seuls les commerçants des kiosques resteront jusqu'à la fermeture à 18 heures. ${ }^{1}$

Le suivi effectué auprès d'un échantillon de maraîchères et de vendeuses du marché pendant une saison maraîchère (décembre à avril) a permis de constater certaines particularités assez originales dans leur comportement de vente. Ainsi, on n'a pu observer de fluctuation notoire dans les prix en ce qui a trait à l'offre et à la demande, au besoin d'écouler un restant de marchandise ou encore à la volonté de réaliser une vente en gros. De plus, et est-ce là l'effet de l'habitude à de constantes pénuries, leur ignorance du calcul, ou encore la fragilité de la mentalité mercantile des vendeuses peu habituées à cette pratique, on constate qu'elles se refusent systématiquement et obstinément à rechercher la monnaie nécessaire à la conclusion d'une vente lorsque le client n'a pas la somme exacte, cette tâche revenant au client et non au commerçant! 2

Par contre, le client semble pour sa part, perméable aux fluctuations des prix, des quantités offertes et de la qualité du produit. 3

D'une manière générale, on a remarqué qu'au niveau de la vente, les ethnies mancagnes et celles de religion musulmane (essentiellement Mandingues), ont une très forte propension au commerce. ${ }^{4}$ Les Mancagnes ont ainsi semblé très enclins à vendre leur production maraîchère alors que les Mandingues vendent et revendent toutes sortes de produits.

\footnotetext{
1 Ibid, pages 6-7.

2 Ibid, pages 78-79.

3 Ibid, page 79.

4 Ibid, page 77.
} 
Soulignons enfin, et pour en quelque sorte appuyer ce que nous avons déjà noté, qu'en règle générale, tous les produits vendus à Bolama sont très dispendieux, surtout ceux provenant de l'extérieur de lî̀le et qui se vendent souvent beaucoup plus chers qu'à Bissau. Par contre, les produits locaux coûtent moins cher à Bolama qu'à Bissau et l'indice de la vie y est moins élevé. Bien que le cours du peso n'a en soi que peu de signification dans une quelconque comparaison entre les coûts de la vie dans différents pays, puisqu'il a longtemps été surévalué ${ }^{1}$ alors que le travail du bissau-guinéen a été sous-évalué, le coût de la vie est aux yeux de la population locale, prohibitif, ce qui ne peut que l'encourager à rester dans le système économique traditionnel. A preuve le fait qu'en 1988, alors que le cours du peso était de 973 P.G. pour un dollar américain, ${ }^{2}$ le salaire mensuel d'un employé du gouvernement, délégué régional du plan, était de 27283 P.G., celui d'un délégué sectoriel de 23309 P.G. 3 et celui d'un travailleur (domestique, employé du privé, etc.) pouvait osciller entre 7000,10000 et 15000 P.G.. Un oignon moyen de 75 grammes coûtait 300 P.G., soit $60 \%$ du salaire quotidien du travailleur rétribué à 10000 P.G. par mois. Le prix du riz, dépassait les 200 P.G. le kilo, ce qui, en tenant compte de la consommation annuelle moyenne de riz du guinéen qui se chiffre à 110 kilogrammes, fait monter les besoins mensuels de riz à près de 2000 P.G. par personne, un oeuf coûtait 100 P.G. et un kilo de poisson, 700 P.G. ... Tous ces prix, en vigueur au début de l'année 1988, n'ont fait que grimper au cours de l'année, diminuant d'autant plus le pouvoir d'achat de la population.

\footnotetext{
1 A titre d'exemple, les devises transigées sur le marché noir peuvent atteindre trois fois la valeur officielle.

2 Banco Nacional da Guiné-Bissau, Câmbios, Bissau, 1-02-88.

3 Departamento de Informatica, Tabela salarial 1988, Bissau, 1988.
} 
3.7 Autres activités économiques

A ce bref survol de l'activité économique bolamienne (agriculture, pêche, cueillette, commerce), il convient d'ajouter l'existence de deux entreprises d'Etat qui ont tout de même une certaine influence au niveau de Bolama, ne serait-ce que par l'emploi qu'elles procurent, Titina Sila et l'Imprimerie Nationale.

\subsubsection{Titina Sila}

L'histoire de cette entreprise de compote et de jus de fruit, construite en 1977 grâce à l'assistance de la Hollande, ne représente qu'une suite de déboires consécutifs à une mauvaise planification et à un désir de la part du gouvernement de l'époque d'entreprendre de grands et prestigieux projets de développement industriel.

Construite au coût de 2 millions de dollars américains, l'usine a dû connaître une fermeture temporaire en 1978 afin d'apporter des correctifs à certains problèmes techniques, dont la grandeur excessive de la presse par rapport aux fruits locaux et l'emballage inadéquat du jus de fruits. Malgré un investissement supplémentaire de 1,5 $\$$ millions qui a amené un certain optimisme dans les milieux intéressés, notamment chez Côté et Demers, ${ }^{1}$ force est d'admettre que l'usine représente un fiasco monumental.

L'entreprise n'a fonctionné normalement qu'en 1979 avec une production de 400000 unités, soit $80 \%$ de sa capacité. ${ }^{2}$ Il faut dire qu'aux niveaux technique et logistique, les difficultés sont énormes. Ainsi, au manque de personnel qualifié à tous les niveaux, s'ajoute la baisse des réserves d'eau douce de l'île en avril et mai, époque du cajou et des mangues,

1 Marthe Côté, André Demers, Repères pour un développement rural intégré dans la région de Bolama. Guinée-Bissau, on cit, pages 141-144.

2 V. Harris, Guinée-Bisssau: développement intégré des îles Biajgos: rapport de mission inter-agences, New York, Nations-Unies, avril 1982, page 33. 
deux des principaux fruits, avec l'orange et le citron, ayant fait partie des plans initiaux de production. ${ }^{1}$ L'approvisionnement en bocaux, en sucre et en carburant pour le bateau et la génératrice, pose aussi des problèmes de taille. L'écoulement de la production cependant, et compte tenu des contraintes déjà mentionnées, peut être envisagé sous un angle positif, car l'absence de concurrence en Guinée-Bissau comme dans les pays voisins dans la production de jus et de compote de cajou pourrait jouer en sa faveur. ${ }^{2}$

Lors d'une visite des installations en avril 1988, nous avons constaté l'état plus que précaire de cette entreprise de 36 employés où, depuis 1986, la production de jus est paralysée, faute d'argent, par le bris d'une pièce d'équipement valant 30000 escudos! 3 Quant à la production de compote, elle fonctionne tant bien que mal, l'usine étant perpétuellement dépendante de produits importés: sucre, pecticine, pots, carton pour les boîtes et pièces de rechange. En 1987, Titina Sila a reçu un financement de la Banque Nationale de Guinée-Bissau de 20 millions de pesos; à la fin de 1987, les 4 millions qui lui restaient lui ont été confisqués, tout comme son bateau et son camion, et le directeur a été démis de ses fonctions pour cause de mauvaise gestion. Titina Sila se retrouve donc en plus mauvaise posture que jamais, avec une rentabilité des plus minimes, de l'ordre de 1500 P.G. la caisse de 20 pots de compote de cajou.

\footnotetext{
1 Marthe Côté, André Demers, op cit, pages 142-143.

2 Ibid, page 142.

3 Soit 223,56 $\$$ canadiens au taux d'octobre 1989 où $1,00 \$$ canadien égale 134,19 escudos (La Presse, Montréal, samedi le 30 septembre 1989).
} 


\subsubsection{L'Imprimerie Nationale}

L'Imprimerie Nationale date de plus de 100 ans et emploie 54 personnes. ${ }^{1}$ Ses activités se bornent à la reliure et à la publication du "Boletim Oficial", organe d'information des activités de l'Assemblée Nationale Populaire, et de quelques feuillets et livrets. Sa situation financière est peu reluisante. ${ }^{2}$ De plus, l'entreprise connaît d'importants problèmes d'entreposage vu les difficultés de transport propres à l'île. Enfin, son générateur n'est pas suffisamment puissant pour permettre le fonctionnement de ses deux modules de production, l'un datant de sa fondation et l'autre ayant été transféré de Bissau en 1978. ${ }^{3}$

Les deux grandes entreprises de Bolama sont donc mal en point, mais pas autant cependant que l'usine de tissage de pagne, construite à la même époque que Titina Sila mais qui attend toujours de produire ses premiers tissus, et dont l'approvisionnement en matière première, le coton, serait dépendante des régions de l'intérieur du pays et du réseau de transport national fortement déficient.

\subsection{Les projets de coopération internationale}

L'île de Bolama est le siège de trois projets liés à la production et d'un projet d'assistance technique à la Délégation Régionale du Ministère du Plan. Ils se caractérisent tous par leur création récente et deux d'entre eux, les deux projets du C.E.C.I., n'ont pas dépassé leur première phase. On note aussi la présence d'assistance médicale dont nous ne faisons que souligner l'existence puisqu'elle ne cadre pas directement avec l'objet du mémoire. Quant à une autre intervention de type "assistance technique", celle du forage de puits, nous nous limitons à souligner sa présence, celle-ci n'étant que ponctuelle. Ainsi,

\footnotetext{
1 Teté João Barai, Diagnóstico do sector de Bolama, op. cit., page 23.

2 V. Harris, op.cit, page 34.

3 Marthe Côté, André Demers, op cit. pages 143-144.
} 
l'appui à l'hôpital régional de Bolama se poursuit toujours avec la relève fournie en 1988 par deux médecins et deux infirmières cubains, à l'équipe de médecins et infirmières de GuinéeConakry en poste depuis plus de cinq ans comme nous l'avons précédemment noté. Pour ce qui est du forage de puits couverts et de l'installation de pompes manuelles, notons que l'intervention était supervisée par la coopération hollandaise grâce à son projet basé à Buba, sur le continent. Ce projet s'est soldé par l'installation de 26 puits en 1987, répartis dans les villages de l'île.

\subsubsection{D.P.R. Bolama-Bijagos}

L'appui à la Délégation Régionale du Ministère du Plan de Bolama-Bijagos (D.P.R. Bolama-Bijagos) ${ }^{1}$ s'est concrétisé par l'envoi d'un "assistant-technique" du C.E.C.I. (Centre Canadien d'Etudes et de Coopération Internationale) de 1986 à 1988. Ayant comme objectif de faire du D.P.R. Bolama-Bijagos un "centre d'information, de coordination et de planification socio-économique sur le milieu", 2 l'intervention dut d'abord et avant tout composer avec un pressant besoin de formation des ressources humaines aux exigences de leurs postes de délégués sectoriels (un pour chacun des quatre secteurs) et de délégué régional (un seul, basé à Bolama). Les besoins en formation du personnel de la délégation (appellation locale pour "delegacia", l'équivalent du département) étaient davantage criants que le personnel ne possédait qu'une formation académique des plus limitées. Ainsi, à Bolama, par exemple, le délégué régional n'avait comme formation scolaire qu'une quatrième année du

1 D.P.R.-Bolama-Bijagos: Departamento de Planificação Regional de Bolama-bijagos (Département de Planification Régionale de Bolama-Bijagos), représentant régional du MICEPCI ("Ministério da Coordenação Económica, da Planificação e da Cooperação Intemacionale", Ministère de la Coordination Economique, de la Planification et de la Coopération Internationale) pour la région.

2 Alain Rousseau, Rapport final d'activité. Projet d'appui à la Délégation Régionale du Ministère du Plan de Bolama-Bijagos: juillet 1986 a inillet 1988. Bolama, C.E.C.I., juin 1988, page 3. 
Cours de Base Elémentaire alors que le délégué sectoriel en était à compléter sa sixième année par les cours du soir pour adultes. Les effectifs des différentes Délégations Régionales du Plan sont encore plus sous-formées que ceux des autres ministères et, chez eux comme dans l'ensemble de la fonction publique bissau-guinéenne, les postes sont souvent accordés sur la base du mérite et des services rendus à la patrie lors de la guerre de libération, plutôt que sur celle de la compétence. Il en découle que les postes clés sont régulièrement tenus, et ce surtout en région, par l'ancienne garde très peu polyvalente et formée à l'économie politique enseignée en U.R.S.S., comme le souligne Carlos Lopes qui prétend que de nombreux "départements de l'Etat sont dirigés par des personnes notoirement incompétentes".1

Ce projet de deux ans a atteint de façon satisfaisante ses objectifs compte tenu des difficultés de financement encourues et de la limitation de ses activités aux seuls secteurs de Bolama et de Bubaque, les deux autres secteurs, Uno et Formosa (Caravela), n'étant que très peu touchés. L'atteinte de l'objectif de l'intervention s'effectua par la réalisation de sept tâches principales, véritables noeuds du projet: ${ }^{2}$

- compiler les différentes données socio-économiques sur la région;

- créer et actualiser un centre de documentation ouvert aux différents intervenants nationaux et étrangers;

- assurer le suivi et la coordination des différentes activités régionales en matière de développement;

- promouvoir les initiatives locales;

- participer au processus national de planification (préparation du Deuxième Plan Quadriennal de Développement Economique et Social);

1 Carlos Lopes, "Ethnie, Etat et rapports de pouvoir en Guinée-Bissau", Itinéraires. notes et travaux, No. 22, mars 1982, Première Edition, Genève, page 67.

2 Ibidem. 
- appuyer techniquement le processus de régionalisation du système de planification national; et,

- former le personnel de la Délégation.

L'étude des structures de planification et, d'une manière plus générale, la deuxième partie du mémoire, relèvera plus en détail certaines facettes de cette intervention, le D.P.R. devant, en théorie, occuper une fonction stratégique dans le développement du pays.

\subsubsection{Le Projet de Développement Agricole Bolama-Bijagos}

Tout comme l'appui au D.P.R. Bolama-Bijagos, le Projet de Développement Agricole Bolama-Bijagos du C.E.C.I. est directement issu de la vaste étude de S.U.C.O.-C.R.A.D., "Le développement rural intégré de la région de Bolama, République de Guinée-Bissau" ${ }^{1} \mathrm{du}$ début des années 1980. Bien que le projet ait officiellement débuté en avril 1985,2 ce n'est qu'en septembre de la même année que le premier expatrié est arrivé à Bolama. ${ }^{3}$ Disposant d'un budget total de $952601,00 \$$ canadiens, ${ }^{4}$ le financement était assuré pour une période d'un peu plus de trois ans et s'étendait d'avril 1985 à septembre 1988, réparti ainsi:5

- avril 1985 à septembre 1986: 494 871,00 \$;

- octobre 1986 à septembre 1987: 197 000,00 \$; et,

1 S.U.C.O.-C.R.A.D., "Le développement rural intégré de la région de Bolama. République de GuinéeBissau", Volumes I, II et III, op.cit.

2 Juan E. Gonzales, Etienne Lamy, Projet de développement agricole dans les îles de Bolama-Bijagos. République de Guinée-Bissau: Evaluation an II. Programmation an III, s.l., Programme Etudes et Projets de Développement Rural, C.E.C.I., janvier 1988, page 4.

3 Paul Rouillard, Hannes Stegemann, Intégration du projet agricole CECI au projet Ilha de Paz, Bolama, C.E.C.I./lle de Paix, 20 avril 1988, page 2.

4 Pierre Thiran, Projet de Développement Agricole dans les Iles de Bolama-Bijagos. République de GuinéeBissau: rapport d'activités, janvier-février-mars 1986, s.l., C.E.C.I., Programme Etudes et Projets de Développement Rural, mai 1986, Annexe.

5 Ibidem. 
- octobre 1987 à septembre 1988: $260730,00 \$$.

L'objectif initial, qui était d'"améliorer la productivité agricole en général" de l'archipel "et plus particulièrement celle de la riziculture",1 se composait de deux volets principaux soit l'appui aux producteurs locaux et celui à l'encadrement technique officiel, le Ministère du Développement Rural (M.D.R.) de la région Bolama-Bijagos. Cet objectif se divisait comme suit:2

-appui aux producteurs locaux:

- accès des producteurs à la technologie appropriée;

- usage du crédit agricole en nature;

- soutien des activités non-agricoles périphériques en amont et en aval de la production agricole en général.

-appui à l'encadrement technique officiel du M.D.R. de Bolama:

- conseil technique et administratif à la restructuration des services techniques du M.D.R. de Bolama;

- formation du personnel technique de base en animation et vulgarisation agricole;

- appui technique à la structure minimale de rechercheexpérimentation agricole.

Le projet a dans un premier temps concentré ses efforts à lîle de Bolama, y affectant un agronome et un chef de projet, et à celle de Bubaque avec l'envoi d'un technologue polyvalent et un agronome. A partir de 1987-1988, le projet a étendu ses activités à l'île de

\footnotetext{
1 Hugues Albert, Serge Blais, Marthe Côté, Pierre Thiran, Projet de développement agricole dans les îles Bolama-Bijagos. République de Guinée-Bissau, op cit, page 98.

2 Ibidem.
} 
Uno avec la venue d'une agronome. A Bubaque, les efforts se sont portés vers la ferme de recherche régionale du M.D.R., la culture du riz pluvial, la lutte contre les prédateurs, principalement le "djiguindor" ou rat de Gambie, et la fabrication de l'huile de palme. A Uno, l'accent a été mis sur la culture du riz d'eau douce. A Bubaque, comme à Bolama, on note la formation d'équipes de vulgarisateurs agricoles qui, équipés de bicyclettes, doivent apporter aide, conseil et appui technique aux paysans.

A Bolama, le projet s'est attardé tout particulièrement à la vente aux paysans de petit matériel de production agricole (machettes, houes, faucilles, etc.). Au niveau de la production de riz, on note la réalisation d'une enquête agricole, l'introduction de variétés de semences à cycle plus court, la réfection des digues de deux rizières et les nombreux efforts de vulgarisation au niveau des soins aux pépinières et des techniques de repiquage. Pour sa part, la production maraîchère a surtout été marquée par la vente de semences et l'encadrement de 130 maraîchères. ${ }^{1}$

Une mission d'évaluation effectuée après la deuxième année de fonctionnement a apporté quelques modifications aux objectifs et aux différents volets du projet. Ainsi, l'objectif initial qui visait l'amélioration de la productivité agricole a été transformé en un objectif plus global aspirant à "améliorer les conditions de vie des paysans des îles de l'archipel (en particulier Bolama, Bubaque et Uno) par le renforcement des activités agricoles". ${ }^{2}$ Le nombre de volets est passé de deux à trois: l'appui aux producteurs et productrices agricoles, l'appui aux services agricoles et l'appui à l'organisation économique qui doit s'effectuer en favorisant l'introduction ou l'amélioration de techniques de transformation et de conservation des produits locaux et en soutenant, par le regroupement de

\footnotetext{
1 Paul Rouillard, Hannes Stegemann, op.cit., pages 3-4.

2 Juan E. Gonzales, Etienne Lamy, op. cit. page 5.
} 
producteurs, la commercialisation de la production. ${ }^{1}$ Ce dernier volet s'avère le complément logique de tout effort de développement agricole qui, une fois la production agricole bien engagée, doit veiller à son écoulement et à sa vente. Il trouve sa source dans les particularités mêmes de la région:

"Ce volet se concentre sur les activités situées en amont [sic] de la production agricole. Depuis de nombreuses années, l'économie de l'archipel se caractérise par un faible volume de transactions commerciales et l'usage très limité de l'argent comme moyen d'échange. Afin de suivre les changements amorcés récemment, les questions de commercialisation d'éventuels surplus agricoles, sans négliger les aspects de conservation et de transformation des productions locales, deviennent des moyens importants pour un développement harmonieux de la région." 2

Le financement du projet n'était assuré que jusqu'en septembre 1988. Compte tenu des contraintes budgétaires canadiennes et de la diminution des sommes consacrées à l'aide au développement international, ${ }^{3}$ le projet n'a pu disposer du financement pour une éventuelle seconde phase. Toute recherche de financement autre que canadien n'ayant pu aboutir, le Projet de Développement Agricole Bolama-Bijagos dut mettre fin à ses activités pour se retrouver dans ce que certains qualifient d'amorce de désengagement, ${ }^{4}$ terme qui laisse supposer que les objectifs du projet ont été atteints. Ce désengagement peut cependant paraître nettement trop hâtif. La réalisation du troisième volet, si nécessaire à un développement harmonieux de la région, aux dires mêmes des représentants de la mission d'évaluation, n'a pu être qu'en partie amorcée. Ainsi, l'appui à l'organisation paysanne pour la commercialisation de la production, et le développement de méthodes dé conservation de la

\footnotetext{
1 Ibid., pages 17 et 23.

2 Juan E. Gonzales, Etienne Lamy, op. cit, page 23

3 Notons que le budget canadien de 1989 a diminué de $360 \$$ millions par rapport à l'année précédente, les fonds fédéraux destinés à l'aide au développement international (Progrès-Dimanche, Chicoutimi, $1^{\circ}$ octobre 1989, "Projets reliés au Tiers-Monde; le dernier budget Wilson fait mal"2.

4 C.E.C.I., Rapport annuel 1988-89, Montréal, C.E.C.I., juin 1989, page 6.
} 
production agricole n'auront pu avoir lieu dans le cadre de ce projet régional. Seule l'étude sur la commercialisation des produits maraîchers aura été effectuée. ${ }^{1}$ Il en découle donc, de l'avis de certains, que les résultats obtenus jusque là auprès des paysans par le projet, peuvent être remis en question par un désengagement hâtif:

"Le projet possède un financement assuré jusqu'au 30 septembre 1988. Malheureusement, le gouvernement canadien ne peut assurer la poursuite du financement total du projet après cette date. Sur le terrain, il serait très déplorable et dommageable pour la dynamique paysannerie - projet que ce dernier cesse ou même s'interrompe pour une période de 6 ou 12 mois. C'est pourquoi nous avons pensé qu'il serait possible d'intégrer la partie du projet CECI touchant Bolama-São-João à l'intérieur du projet ONG belge "Les lles de Paix" qui a manifesté un vif intérêt pour cette éventualité." 2

Il convient de noter l'envoi de coopérants-volontaires afin de substituer à un projet d'envergure régionale, un certain nombre de petits projets locaux, qui, vu les risques de morcellement et le piège de l'isolement propre au travail dans un tel archipel, ne pourront aspirer à l'aspect global qu'un véritable projet régional pouvait assurer. Il apparait que la transformation d'un grand projet en petits projets moins coûteux constitue certes un palliatif à un manque de fonds, de même que l'assurance de l'occupation du territoire par l'organisme, ce qui ne fait que confirmer, d'un autre côté, l'état de fait d'un désengagement trop hâtif imposé par des contraintes essentiellement financières.

\subsubsection{Le projet de pêche Pescarte/Ittica Valdagri}

Initialement prévu pour s'implanter dans la région de Biombo, ce projet de pêche, encadré par une firme privée italienne spécialisée dans l'aquaculture, Ittica Valdagri Spa, a été

\footnotetext{
1 Brigitte Boies, Alain Rousseau, Enquête sur la commercialisation des produits maraîchers, on. cit., 80 pages.

2 Paul Rouillard, Hannes Stegemann, op_cit., page 4
} 
transféré à Bolama vu l'existence d'un autre projet de pêche au Biombo et la situation catastrophique de la pêche à Bolama. Le projet se retrouve en outre sous la responsabilité de Pescarte, abréviation de "Pesca Artesanal" (pêche artisanale), relevant du Secrétariat d'Etat aux Pêches et, plus spécifiquement de la Direction de la Pêche Artisanale et des Projets de Développement, dont les tâches s'appliquent au développement de la pêche nationale et de la commercialisation de ses produits. Le projet a tout d'abord reçu un financement de $1,5 \$$ millions américains pour une période de 18 mois, d'avril 1987 à septembre 1988, provenant des budgets d'aide d'urgence du ministère italien des Affaires Etrangères via le F.A.I. ("Fundo de Adjuda Italiana" Fonds d'Aide Italienne). ${ }^{1}$

Le projet avait l'ambitieux objectif de former le personnel et les cadres locaux de Pescarte-Bolama, les pêcheurs et une équipe de mécaniciens afin, qu'après 18 mois d'intervention, ceux-ci soient en mesure de faire fonctionner le projet de façon autonome. ${ }^{2}$ Les choses ne se sont pas passées aussi facilement et rapidement, et on n'a procédé à l'ouverture officielle du projet qu'en janvier $1988 .{ }^{3}$ Les premiers effets du projet n'ont commencé à se faire sentir qu'à partir de cette époque: vente de matériel aux pêcheurs, capture de poisson, etc.

L'équipe sur place compte un chef de projet, un pêcheur et un mécanicien, tous expatriés. Du budget initial de 1,5 \$ millions, plus de $45 \%$ est constitué de matériel divers pour les logements et les bureaux, et de matériel de pêche destiné à la vente. Les interventions principales du projet concernent la production de glace à partir de l'eau de mer, la mise sur pied d'un atelier mécanique et d'un atelier de menuiserie, et la vente par crédit de 30 moteurs

\footnotetext{
1 Entrevue auprès de Stefano Moretti, coordonnateur de Ittica Valdagri à Bolama, le 25 février 1988.

2 Ministério dos Negocios Estrangeiros da Républica Italiana, Sintese do Projecto Centro de Pesca Artesanal em Bolama. Guiné-Bissau, s.l., Ittica Valdagri, Serviço Especial Intervençðes Extraordinárias nos Países em vias de Desenvolvimento, pages 15-16.

3 Entrevue auprès de Stefano Moretti, op. cit.
} 
hors-bords au diesel et de matériel de pêche. On prévoit remettre aux autorités locales de Pescarte, l'ensemble des biens et du matériel importé à partir d'octobre 1988. ${ }^{1}$

Le bilan de cette première intervention en matière de pêche à Bolama a nettement démontré que l'atteinte de l'objectif de relance de la pêche n'est pas possible en un si court laps de temps et ce, compte tenu des contraintes propres au milieu, tant au niveau de l'approvisionnement et du transport qu'au niveau des pêcheurs qui s'avèrent très lents à s'intégrer dans tout processus. Peu à peu, l'objectif du projet s'est fait plus clair et a cerné les différentes facettes nécessaires au renforcement de la pêche à Bolama, en dégageant le besoin de fournir plus de moyens et d'autonomie à la pêche notamment en s'intégrant au niveau du commerce et de la distribution, ce que les intervenants étrangers ne comptaient pas faire initialement, contrairement aux cadres de Pescarte. ${ }^{2}$ Tout ceci a commandé un financement supplémentaire de $5 \$$ millions américains, qui leur fut accordé et ce, pour une période de quatre ans, c'est-à-dire jusqu'en 1992.

Les sommes investies dans le projet dénotent clairement l'esprit qui anime cette intervention, favorisant le développement de la pêche commerciale à coups d'argent et de matériel. L'opinion populaire face au projet fait état d'une certaine méfiance, à preuve ces pêcheurs artisanaux qui nous affirmaient ne pas vouloir travailler avec Pescarte, ce dernier les forçant à y vendre leurs prises et ainsi à s'intégrer dans un processus de production et de remboursement de matériel acheté dont ils ne veulent pas vraiment, de peur de perdre leurs habitudes ancestrales de culture de riz et de pêche d'autosuffisance. ${ }^{3}$ On peut d'ailleurs s'interroger sur la capacité d'absorption sans heurts, de la part de la population bolamienne,

${ }^{1}$ Hannes Stegemann, Ile de Paix de Bolama, rapport de mission à Bolama. 03/04/87-23/05/87, op. cit, page 11.

2 Entrevue auprès de Stefano Moretti, op. cit.

3 Entrevue avec deux membres du comité de tabanca du village de Madina, Colly Baldo et Bubal Baldo, le 11 novembre 1987. 
faible en nombre et retranchée dans un mode de vie traditionnel pour la majorité, d'un capital si important, en un temps si court...

\subsubsection{Ile de Paix}

L'O.N.G. belge les "Iles de Paix" 1 implanta, à partir de 1986, un projet à deux volets sur l'île de Bolama, soit l'installation d'un atelier de menuiserie et la production de quatre embarcations en bois pour le compte d'un autre projet basé à Bubaque, le P.D.I.I.B. (Projet de Développement Intégré des Iles Bijagos) du P.N.U.D. (Programme des Nations-Unies pour le Développement). Cette première phase, que l'on peut, dans le cas d'lle de Paix, qualifier de phase d'implantation, avait comme objectifs principaux l'apport à l'amélioration des communications entre les îles par la construction de bateaux, la formation d'un groupe d'artisans locaux capables de construire et de gérer la production de ce type de bateaux et, enfin, la mise en place d'une infrastructure à long terme nécessaire au développement futur du projet. Ainsi, de février 1986 à la fin de l'année 1987, Ile de Paix s'est concentrée à la réalisation de ses objectifs 2 avec une équipe d'une vingtaine d'employés nationaux composée de menuisiers, peintres et logisticiens, et de quatre coopérants étrangers, essentiellement un menuisier, un mécanicien, une anthropologue et un chef de projet. Le budget alloué à ce projet est de 253277 E.C.U. en 1986, 275427 E.C.U. en 1987 et 300000 E.C.U. en 1988, le tout financé à part égale par les Iles de Paix et la C.E.E. ${ }^{3}$.

\footnotetext{
1 Il est courant de parler de lO.N.G. au pluriel alors que l'on se réfere aux projets sur le terrain au singulier. Par conséquent, Iles de Paix désigne le siège social en Belgique alors qu'lle de Paix concerne le projet de Bolama (note de l'auteur).

2 Paul Rouillard, Hannes Stegemann, Intégration du projet agricole CECI au projet Ilha de Paz, op. cit., page 1; et, Hannes Stegemann, op.cit, pages 6-7.

3 D.P.R.-Bolama-Bijagos, Inquérito dos projectos, op. cit..
} 
Le bilan de la phase I s'avère positif, si l'on excepte le fait que les embarcations construites, dont le prix de revient est de $18985,30 \$$ américains ${ }^{1}$ auquel il convient d'ajouter 4000 américains pour le moteur, ${ }^{2}$ coûtent nettement trop cher pour la population locale. Ceci met en cause les objectifs initiaux du P.D.I.I.B. qui étaient de créer et d'équiper des équipages locaux pour le transport de marchandises et de personnes, afin de former une association de transporteurs. ${ }^{3}$ On remarque même que le fait que $91 \%$ des dépenses de construction aient été effectuées en devises, pose un problème de taille pour la prise en charge de telles constructions par des artisans locaux. Quant à la technologie employée, elle s'avère elle aussi inadaptée au contexte local car l'utilisation conjointe de la voile et du moteur est trop complexe pour les équipages de l'archipel. ${ }^{4}$ De même convient-il de souligner au plan des faiblesses de la première phase du projet, l'impossibilité, vu le rythme de travail imposant découlant d'un calendrier de production surchargé, d'avoir pu fournir les efforts nécessaires à la formation des travailleurs de l'équipe de Bolama en matière de gestion. Il en découle la difficulté de rendre les travailleurs autonomes dans leurs fonctions, objectif que l'on visait à moyen terme. 5

Cette première phase, qui en était une d'implantation, ne doit pas faire oublier les objectifs initiaux d'Ile de Paix. En effet, une première mission effectuée en 1984 avait retenu différents secteurs d'intervention possibles comme la pêche artisanale, l'agriculture, la santé et l'approvisionnement de Bolama en eau douce. En 1985, lors d'une seconde visite, on avait retenu l'idée d'une première phase d'implantation, la phase que nous avons effectivement

\footnotetext{
1 Hannes Stegemann, op cit., page 41.

2 Philippe de Braconier, Elizabeth Drory, Rapport de mission de juin 1985; prétude de l'Ile de Paix de Bolama, op. cit., page 27.

3 Hannes Stegemann, op.cit. page 5.

4 Ibid., page 8.

5 Ibid, page 7.
} 
connue. Or, il appert que cette nuance, qui fait que la construction d'embarcations ne constitue pas la finalité du projet mais seulement une phase d'implantation, n'ait pas été très bien retenue ou comprise par les différents ministères à Bissau. Ceci se concrétisa par l'apparition du projet de pêche Pescarte à Bolama, venant en quelque sorte couper l'herbe sous les pieds d'Tle de Paix pour la réalisation de sa deuxième phase qui s'orientait de plus en plus, dans l'esprit de l'organisme, vers la pêche. ${ }^{1} \mathrm{Ce}$ manque de coordination au niveau de l'octroi des interventions dans l'île est directement imputable, de l'avis de l'O.N.G., au Ministère du Plan. Quoiqu'il en soit, le désir d'exercer un certain monopole au niveau des interventions d'Hle de Paix est facilement perceptible et on comprend, qu'à l'origine, l'O.N.G. s'installait à Bolama pour un certain temps et comptait bien y diversifier ses activités 2 et ce, selon un certain monopole.

Il fut donc décidé d'entamer une seconde phase d'une durée prévue de seulement 12 mois, afin d'assurer une planification plus facile et une meilleure flexibilité pour faire face aux vices de la planification et de la concertation dans l'assistance au développement de Bolama par le gouvernement bissau-guinéen et les divers intervenants impliqués. ${ }^{3}$ Les objectifs suivants font donc oeuvre de deuxième phase:4

- consolidation et continuation de la première phase de construction de bateaux;

- installation d'un atelier de mécanique et formation de trois mécaniciens;

- installation d'un groupe de menuisiers autonomes dans les locaux d'Tle de Paix;

- recherches sur la pêche artisanale avec prise de contact avec des pêcheurs locaux et vente de petit matériel de pêche;

1 Ibid., pages 6-7.

2 Entrevue avec Philippe de Braconier, secrétaire général d'Tles de Paix, Bolama, 10 avril 1987.

3 Paul Rouillard, Hannes Stegemann, op.cit. page 2.

4 Ibid., pages 1-2. 
- implication auprès des femmes de Bolama;

- amélioration de certaines infrastructures de la ville; et,

- installation d'un point de vente à lle de Paix (outils, clous, vis, colle, etc.).

Ile de Paix aura ainsi pris la décision de s'impliquer dans la pêche artisanale malgré la présence de Pescarte. Notons que cette initiative dénote l'existence d'un certain travail conjoint entre Ile de Paix et le D.P.R. de Bolama qui, lors de l'enquête de tabanca avait identifié les besoins en matériel de pêche dans les villages de lî̀le. Du côté de Pescarte, l'implication d'Ile de Paix dans la pêche traditionnelle ne semble cependant pas poser de problème, vu la spécialisation de Pescarte dans la pêche professionnelle suivant le but d'approvisionner le marché urbain de Bolama et, éventuellement, de Bissau. Nous pouvons par contre remarquer la fragilité d'un tel équilibre, tant au niveau de deux projets géographiquement voisins et oeuvrant sur un terrain presque commun, qu'à celui de la capacité d'absorption de la population locale qui n'a peut-être pas besoin de deux projets de pêche au même endroit. Il importe donc ici de distinguer les véritables intérêts de la population et ceux de projets voulant chacun avoir leur propre sphère d'influence. 
DEUXIEME PARTIE 


\section{CHAPITRE QUATRE}

\section{LES STRUCTURES POLITICO-ADMINISTRATIVES}

Bien que l'étude du système politique bissau-guinéen ne fasse pas expressément partie des objectifs de notre mémoire, il n'en demeure pas moins important d'en expliquer brièvement la composition afin de pouvoir mieux situer le rôle des différentes structures administratives du pays.

\subsection{Le P.A.I.G.C.}

Le P.A.I.G.C., fondé comme nous l'avons vu en 1956, était d'abord et avant tout un mouvement de libération nationale et, avec l'acquisition de l'indépendance politique du pays, le P.A.I.G.C. est devenu la seule et unique force politique dirigeante. ${ }^{1}$ Lors du Troisième Congrès du Parti en 1977, premier congrès ayant eu lieu dans un contexte d'indépendance nationale, les deux premiers s'étant tenus au cours des années 1964 et 1973, le P.A.I.G.C. fit le point sur les leçons à tirer des années de lutte et des premières années d'indépendance et ce, pour être en mesure d'asseoir les bases politiques et idélogiques nécessaires à cette deuxième phase du progrès national qu'est la recherche du développement de la nation. ${ }^{2} \mathrm{Ce}$

\footnotetext{
1 Lars Rudebeck, Guinea-Bissau: difficulties and possibilities of socialist orientation,Uppsala University, Institute of Political Science ans the Working Group for the Study of Development Strategies (A.K.U.T.), january 1978, pages 1 et 5 .

2 lbid, page 8.
} 
Congrès confirma officiellement les fonctions de domination de l'Etat par le Parti, dans la réalisation du programme national économique, social, culturel et de défense et de sécurité du pays. ${ }^{1}$

Lors de l'ère de Nino Vieira, le Quatrième Congrès (novembre 1986) poursuivait toujours des objectifs similaires et confirmait indiscutablement le rôle prépondérant du Parti au sein de l'Etat. Le thème du congrès était d'ailleurs le renforcement du Parti pour le développement national ("Congresso de Reforço do Partido para o Desenvolvimento Nacional"). ${ }^{2}$ Le rôle dirigeant du P.A.I.G.C. est donc demeuré présent tout comme lors du congrès précédent ${ }^{3}$ et ce, malgré le changement de régime:

"Artesão da vitória do nosso Povo sobre o colonialismo, o PAIGC apareceu na nova etapa como o único garante dos interesses das massas trabalhadoras e de successo na luta pelo desenvolvimento, bemestar e justiça social e pela eliminação da exploração do homem pelo homem, objectivos cuja primeira e mais sublime expressão histórica foi a própria Luta de Libertaçãoo Nacional." 4

Le Parti souligne son implication auprès des masses de travailleurs. In faut se garder d'associer ces travailleurs au prolétariat industriel, générateur de plus-value. En fait, on comprendra que, dans une société avant tout rurale et agricole comme la Guinée-Bissau, on entendra par "masses travailleuses", autant les prolétaires que les paysans eux-mêmes par qui

1 Aristides Pereira, Rapport du Conseil Superieur de la Lutte du PAIGC, présenté au III Congrès du Parti. novembre 1977, cité dans, Carlos Lopes, "Ethnie, Etat et rapports de pouvoir en Guinée-Bissau", Itinéraires. notes et travaux, No. 22, mars 1982, Première Edition, Genève, Annexe, page III.

2 P.A.I.G.C., Teses para o IV Congresso do PAIGC, Bissau, Edição do Departamento de Informação, Propaganda e Cultura do Comite Central do P.A.I.G.C., julho 1986, page 7.

3 Paulette Pierson-Mathy, La naissance de lEtat par la guerre de libération nationale: le cas de la GuinéeBissau, Paris, U.N.E.S.C.O., 1980, page 26.

4 P.A.I.G.C., op. cit., page 7.

"Artisan de la victoire de notre Peuple sur le colonialisme, le PAIGC apparaît, dans la nouvelle étape, comme le seul garant des intérêts des masses travailleuses et de la lutte pour le développement, le bien-être et la justice sociale et pour l'élimination de l'exploitation de l'homme par l'homme, objectifs dont la première et plus sublime expression historique fut la propre Lutte de Libération Nationale" (traduction de l'auteur). 
le succès de la lutte anti-colonialiste a été possible. Ainsi peut-on questionner le fait que certains auteurs portent la critique d'une façon des plus ambivalentes face au rôle et à la définition des travailleurs:

"Ideologically, there is no ambivalence. The PAIGC represents the working people, i.e. the masses of peasants and workers, and all others committed to the goal of national liberation and progress. Theorically, this gives the working class a major role in a long term pespective. But in practice, the working class of Guinea-Bissau is still so small that it can hardly play a decisive political role alone." 1

Pour notre part, la démarche adoptée ne sera pas quantitative. Par conséquent, plutôt que de la faire reposer sur le nombre effectif de prolétaires, nous baserons notre analyse sur la participation ou non des travailleurs (qu'ils soient paysans ou travailleurs-prolétaires) au but final du P.A.I.G.C., le développement d'une société juste, équitable, et à la recherche du bien-être de tous. Nous ne pourrons, du moins l'espérons-nous, porter jugement qu'une fois toutes les données du problème bien en mains, ce que nous nous efforcerons de réaliser dans cette partie du mémoire.

A la recherche d'une société libérée de l'exploitation de l'homme par l'homme, le P.A.I.G.C. poursuit, sur le plan international, une politique anti-impérialiste le poussant à appuyer les diverses luttes de libération dans le monde. De même adhère-t-il à une politique de non-alignement l'entraînant à entretenir une volonté d'établir des liens de coopération avec tous les Etats. I affirme d'ailleurs être dirigé par les principes du centralisme démocratique, de l'autocritique et de la direction collective. ${ }^{2}$ Ces principes, énoncés lors du Troisième

1 Lars Rudebeck, op.cit.page 19.

"Idéologiquement, il n'y a pas d'ambivalence. Le P.A.I.G.C. représente la classe laborieuse, i.e. les masses de paysans et de travailleurs, et tous ceux engagés dans le but de la libération nationale et du progrès. Théoriquement, ceci donne à la classe laborieuse un rôle majeur dans une perspective à long terme. Mais en pratique, la classe laborieuse de Guinée-Bissau est encore trop petite et ne peut que difficilement avoir seule un rôle politique décisif" (traduction de l'auteur).

2 Paulette Pierson-Mathy, op. cit., page 26. 
Congrès sont toujours en vigueur, et il faut leur ajouter, sur le plan interne, l'adoption, ou plutôt la confirmation, au Quatrième Congrès, d'une économie mixte. ${ }^{1}$

Il pourrait être intéressant de considérer ou même d'associer l'objectif du P.A.I.G.C., d'abolir l'exploitation de l'homme par l'homme, aux buts socialistes, malgré que ce terme n'apparaisse clairement dans les déclarations et écrits des membres du Parti que très rarement. En fait, on note qu'Amilcar Cabral lui-même était distant face à l'idée d'associer son mouvement au socialisme, au marxisme ou même au marxisme-léninisme, puisqu'il ne percevait la Guinée-Bissau que comme un pays très peu avancé aux points de vue économique et technologique et, de ce fait, très loin de la recherche immédiate du socialisme. Dans ce cas précis, souligne Rudebeck, l'adoption du langage marxiste par la critique bissauguinéenne relève donc plutôt de considérations propres au besoin de l'emploi d'une grille d'analyse critique à la recherche de l'émancipation de l'homme, ${ }^{2}$ que d'une idéologie révolutionnaire devant aboutir à l'abolition de la propriété privée des moyens de production, à la disparition des classes sociales ou encore à toute autre phase propre à un socialisme précurseur du communisme.

Quoiqu'il en soit, le P.A.I.G.C. véhicule des conceptions propres à tous les mouvements de tendance progressiste, influencés par le mouvement révolutionnaire marxiste et les principes léninistes du Parti. ${ }^{3}$ Ainsi en est-il du rôle dirigeant du Parti sur les structures de l'Etat grâce à un exercice constitutionnel des plus remarquable. Officiellement, en GuinéeBissau, Parti et Etat sont séparés. Dans la pratique, le caractère supérieur du Parti, qui affirme que la répartition du pouvoir qu'il a su effectuer parmi les différentes couches de la société

1 P.A.I.G.C., op.cit., page 38.

2 Lars Rudebeck, op. cil., pages 5-6.

3 Carlos Lopes, A transicão histórica na Guiné-Bissau: do movimento de libertacão nacional ao Estado, Genève, Mémoire présenté pour le Diplôme de Recherche en Etudes du Développement, Institut Universitaire d'Etudes du Développement, novembre 1982, page 169. 
bissau-guinéenne ${ }^{1}$ lui assure la justesse et la représentativité populaire, se traduit par le fait que le P.A.I.G.C. veille à ce que l'Etat exécute ses propres politiques. L'Etat, à l'aide de ses différents organes, joue alors le rôle d'instrument d'exécution des vues du Parti puisque ce dernier ne peut réaliser toutes les étapes techniques, administratives ou autres, nécessaires à la concrétisation des ses objectifs politiques. ${ }^{2}$

\subsection{Structures politiques et administratives}

Les structures politiques et administratives de la Guinée-Bissau correspondent à un modèle théorique des plus intéressants au point de vue de la décentralisation. Ainsi, les structures du Parti partent-elles de la base, les villages ou les quartiers, pour se rendre dans les sections, puis ensuite les secteurs, suivis des régions, pour enfin aboutir au niveau central national, à Bissau, avec le Comité Central (Conseil Supérieur de la Lutte), le Bureau Politique (Conseil Exécutif de la Lutte) et, enfin, la Commission Permanente. ${ }^{3}$

\subsubsection{Les comités de tabanca}

Les structures de base sont appelées comités de tabanca ou encore comités de village ou de base. Ils représentent un village, un quartier ou, lorsque le nombre d'habitants ne le justifie pas, un groupe de villages ou de quartiers. La population élit, en son sein, les cinq membres du comité, par ailleurs membres du Parti, dont au moins un se doit d'être une femme, selon la composition suivante: ${ }^{4}$

\footnotetext{
1 Ibid, page 167.

2 Ibid. page 169.

3 Carlos Lopes, "Ethnie, Etat et rapports de pouvoir en Guinée-Bissau", op. cit., page 65.

4 Anne-Marie Hochet, Paysanneries en attente: Guinée-Bissau, Dakar, Environnement Africain, Série Etudes et Recherches, No. 79-80, janvier-février 1983, page 46.
} 
- un président;

- un vice-president;

- un trésorier,

- un responsable de la santé;

- un responsable de la propreté du village, qui est habituellement en charge de la J.A.A.C. (Jeunesses Africaines Amilcar Cabral, mouvement de jeunes du Parti); et,

- un(e) responsable des femmes.

Ces comités, au nombre de 25 sur l'île de Bolama, correspondent, soit dit en passant, aux villages et quartiers enquêtés lors de l'enquête de tabanca, et sont regroupés en sections. Bolama compte deux sections, celle de Bolama et celle de Bolama de Baixo. ${ }^{1}$

Il importe de comprendre que les comités de tabanca, de par leur caractère artificiel par rapport aux us locaux, occupent une place ambiguë au sein des communautés en ce qui a trait à leur rôle vis à vịs des autorités traditionnelles villageoises. Les avis sont partagés quant à leur rôle et à leur efficacité. Certains perçoivent un esprit de concurrence entre les comités de tabanca et les autorités traditionnelles dans l'exercice de leurs responsabilités respectives. ${ }^{2}$ D'autres pensent que la population a le choix d'élire au sein de ces comités, ceux qui, de toute façon, détiennent traditionnellement le pouvoir, sans quoi le comité, soit n'a aucun pouvoir, soit divise la population entre lui-même et le pouvoir traditionnel. ${ }^{3}$ D'autres enfin, conçoivent l'efficacité et limplication du comité comme dépendant du dynamisme propre de ses membres, car ces comités, éléments extérieurs au village en matière de conception et de

1 D.P.R., Lista nominal das seccōes por sectores administrativos, Bissau, 25 de fevereiro de 1987, page 1.

2 Marthe Côté, André Demers, Repères pour un développement rural intégré dans la région de Bolama. Guinée-Bissau, Université Laval, Maîtrise en Aménagement du Territoire et en Développement Régional, novembre 1982, page 201.

3 Jean Brodeur, Guinée-Bissau, s.l., texte polycopié, septembre 1979, page 4. 
fonctionnement, peuvent être un atout pour le village qui saura y trouver un interlocuteur fiable dans la transmission de ses doléances auprès des autorités gouvernementales: 1

"Dans une optique de développement,[...], nous pensons que les comités de tabanca sont les seules instances locales aptes à prendre des initiatives concrètes et efficaces. Nous espérons encore, en dépit des premières erreurs commises après l'indépendance, que la Guinée-Bissau s'évitera la situation des pays voisins où l'on paie l'erreur d'avoir placé tout de suite un encadrement rural rigide et non formé au lieu de tirer profit des tendances organisationnelles villageoises qui s'expriment à travers les comités de tabanca." 2

Notre position se situe à la croisée de ces trois chemins si l'on peut dire. Il ne fait aucun doute à nos yeux que l'efficience d'un comité et son acceptation de la part des villageois reposeront dans son dynamisme et, celui-ci, comme nous l'aurons maintes fois remarqué, viendra souvent du fait que des membres du comité sont en étroite relation avec l'autorité traditionnelle du village, ou même font partie des autorités traditionnelles: le cas des villages mandingues et foulas à Bolama (Uato Mandinga et Madina) est éloquent à ce sujet. ${ }^{3}$ Dans les autres cas, cependant, les comités de tabanca éprouvent des difficultés certaines, soit par manque de dynamisme, soit par leur faible influence auprès de leur population, et souvent ne savent tout simplement pas ce qu'ils doivent faire, par manque d'encadrement et de formation. Quoiqu'il en soit, nous ne croyons pas judicieux et viable l'opinion de certains fonctionnaires qui voient en ces comités, un organe en pleine ascension, appelé à supplanter inévitablement le pouvoir traditionnel. ${ }^{4}$ Il demeure évident qu'à court terme du moins, vu les graves lacunes en matière de reconnaissance villageoise qu'ils peuvent parfois connaître

1 S.U.C.O.-C.R.A.D., Le développement rural intégré de la région de Bolama. République de Guinée-Bissau, Université Laval, Maîtrise en Aménagement du Territoire et Développement Régional, 1982, Volume I, page 144.

2 Anne-Marie Hochet, op cit. page 48.

3 Entrevue avec Paul Rouillard, chef du Projet de Développement Agricole Bolama-Bijagos, Bolama, le 2 juin 1988.

${ }^{4}$ Entrevue avec Luis Napoleão dos Reis, délégué régional, D.P.R.-Bolama-Bijagos, Bolama, le 25 mai 1988. 
lorsqu'ils ne sont pas directement associés au pouvoir traditionnel, les comités de village ne peuvent aspirer à jouer un rôle véritablement moteur dans leur communauté:

"Mais le comité de "tabanca" n'a pas réussi à ce jour à s'intégrer dans le milieu de façon convaincante; il n'a pas l'autorité suffisante pour imposer des consignes d'action. Ainsi, en 1980, il a été difficile aux comités de Bubaque de faire comprendre le bien-fondé de calculer les productions de riz sur le champ et de mesurer les superficies. Les paysans craignaient que ce calcul serve à prélever un impôt sur la production. En somme c'est plus souvent par obligation et sans conviction que les comités de "tabanca" sont écoutés et leur influence est d'autant plus faible qu'ils n'apportent pas grand chose d'autre que l'imposition des règlements. Jusqu'à maintenant, les comités de base ont été contrôlés par le haut, les comités de secteur étant les véritables lieux de transmission de l'autorité et des doléances des villages. Seuls des projets ou services gouvernementaux organisés et contrôlés à l'échelon local pourraient permettre d'augmenter la crédibilité des comités de base." 1

Les comités de tabanca sont intégrés au pouvoir politique du pays comme le montre le Tableau 4.1, et les cinq représentants sont élus pour une durée de quatre ans. Les fonctions des comités de tabanca vont de la transmission et de l'exécution dans leur village respectif, des consignes politiques et administratives émanant de paliers supérieurs (régionaux ou nationaux), au règlement de la justice dans le village ${ }^{2}$ et à toute tâche d'encadrement, d'organisation ou de mobilisation nécessaire à un quelconque travail. On comprend clairement ici que les comités de tabanca, organes politiques de base du Parti, s'ils ne visent pas à se substituer aux autorités traditionnelles, aspirent tout de même à doter chaque village du pays de structures politiques semblables au service du Parti. Ainsi, comme le souligne Anne-Marie Hochet:

1 S.U.C.O.-C.R.A.D., op.cit., page 144.

2 Il ne s'agit ici en fait que de la justice s'appliquant aux affaires internes du village (conflits de familles, litiges fonciers, rixes, vols, etc.); on ne fait appel à la justice officielle que lorsque, par exemple, un problème ne peut être résolu, car la population a recours le moins possible à la justice extérieure au village ( Anne-Marie Hochet, op cit., pages 46-47). 
TABLEAU 4.1

Structures politico-administratives

Guinée-Bissau

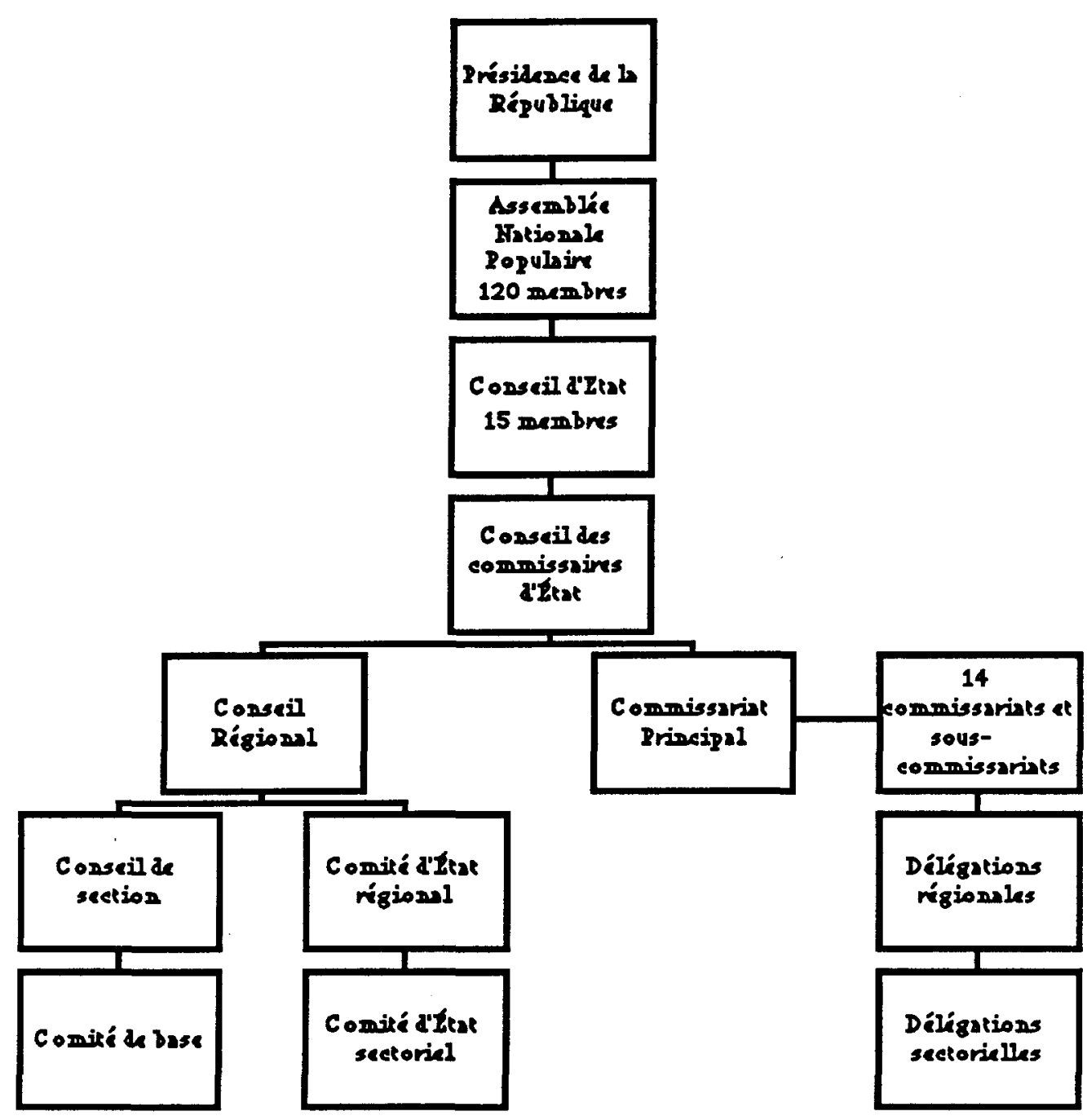

Source: Carlos Lopes, A transicão histórica na Guiné-Bissau: do movimento de libertação nacional ao Estado, Mémoire présenté pour le Diplôme de Recherche en Etudes du Développement, Genève,Institut Universitaire d'Etudes du Développement, novembre 1982, page 97;

Paulette Pierson-Mathy, La naissance de l'Etat par la guerre de libération nationale: le cas de la Guinée-Bissau, Paris, U.N.E.S.C.O., 1980, page 140;

Lars Rudebeck, Guinea-Bissau: difficulties and possibilities of socialist orientation, Institute of Political Science ans the Working Group for the Study of Development Strategies (A.K.U.T.), Uppsala University, january 1978, page 18; et, Ajout par l'auteur des délégations régionales et sectorielles. 
"Il nous semble donc que ces comités de tabanca, tels qu'ils sont et surtout tels qu'ils fonctionnent, reflêtent le village et ses tendances organisationnelles, tout en assurant à l'administration un relais concret entre elle-même et le village." 1

A partir des comités de tabanca regroupés en sections, la population élit, à tous les quatre ans, un député (membre d'un comité) par section pour siéger au conseil régional. ${ }^{2}$

\subsubsection{Le pouvoir régional}

La loi électorale du pays accorde le droit de vote à toute personne, des deux sexes, de 18 ans et plus, exempte de démence mentale et n'ayant commis aucun crime. ${ }^{3}$ La population ne fait preuve que d'un intérêt mitigé face à l'exercice de son droit de vote, comme le prouve le faible taux de participation à l'échelle du pays. En 1976, par exemple, le taux de participation était à peine de $50 \% .^{4}$

Le conseil régional constitue le dernier palier où s'appliquent les principes du suffrage universel. Son rôle est d'approuver le programme annuel de développement de la région, dont la préparation incombe au comité d'Etat régional, de veiller à la réalisation des activités régionales faisant partie du plan national, d'approuver le budget régional et de prendre connaissance de l'état des comptes régionaux..$^{5}$

L'organe exécutif est le comité d'Etat régional qui a son équivalent au niveau des secteurs avec le comité d'Etat sectoriel. Le comité d'Etat régional est composé d'un président,

\footnotetext{
1 Anne-Marie Hochet, op. cit, page 48.

2 Entrevue avec Luis Napoleão dos Reis, op. cit..

${ }^{3}$ Republica da Guiné-Bissau, Boletim Oficial, No. 48, quarta-feira, $1^{\circ}$ de dezembro de 1976.

${ }^{4}$ Lars Rudebeck, Guinea-Bissau: difficulties and possibilities of socialist orientation, op.cit., page 19.

5 Isabel Sarmento, Análise do sub-sistema de planeamento e da administracão regional da Republica da GuinéBissau, s.l., 1985, page 10.
} 
nommé par le Conseil des Ministres, de deux représentants du conseil régional élus parmi ses membres, de trois citoyens élus par les comités de base, et des délégués des ministères en région. Le comité d'Etat du secteur est, pour sa part, composé d'un président, d'un viceprésident, de quatre délégués des ministères et de deux citoyens élus par les comités de base. ${ }^{1}$

Le comité d'Etat régional a pour principales fonctions d'exécuter les résolutions adoptées par le conseil régional, de préparer le programme annuel de développement de la région, de diriger les services administratifs, de contrôler les entreprises locales et d'appuyer les activités des diverses entreprises et services en région. ${ }^{2}$

Il fut établi, dans la nouvelle constitution du 16 mai 1984, que le conseil régional et le comité d'Etat régional composaient les organes du pouvoir local, ${ }^{3}$ et que les décisions des conseils régionaux primeraient sur toutes les institutions, collectivités ou citoyens de la région. ${ }^{4}$ En pratique cependant, on note la prédominance du comité d'Etat au sein de la région car, outre son pouvoir exécutif, son président, gouverneur administratif et chef des organisations du Parti en région, ${ }^{5}$ s'avère en fait être l'autorité suprême régionale, comme nous le verrons à plusieurs reprises.

\subsubsection{Le pouvoir administratif}

En Guinée-Bissau, la terminologie employée au niveau administratif, tout comme les pouvoirs dévolus aux différents paliers du gouvernement, ont tendance à changer souvent, au

\footnotetext{
1 Ibid., pages 10-13.

2 Ibid., page 10.

3 Ibid, page 9.

4 Paulette Pierson-Mathy, La naissance de l'Etat par la guerre de libération nationale: le cas de la GuinéeBissau, op. cit., page 135.

5 Lars Rudebeck, op, cit., page 18.
} 
gré apparent de nombreuses et diverses réorientations. Ainsi a-t-on vu l'appellation des ministères passer de commissariat et sous-commissariat d'Etat au début de la République, à commissariat d'Etat en 1975, puis à secrétariat d'Etat au début des années 1980, pour se diviser, au milieu des années 1980 , en ministère et secrétariat d'Etat. ${ }^{1}$ Ces nombreux changements obligent à constamment relativiser et réviser le langage employé et les fonctions et attributions de chaque niveau de gouvernement. Il ne faut donc pas trop s'attarder et s'étonner des quelques différences et nuances que l'on pourrait constater au long de cette partie du mémoire, en ce qui a trait aux structures étatiques. De même ne faudra-t-il pas faire cas de l'appellation donnée aux ministères, comme par exemple le Ministère du Plan, que l'on désignera sous cette dénomination plutôt que toute autre (C.E.C.E.P., MICEP, MICEPCI, etc.), si ce n'est celle de délégation pour identifier le pendant régional ou sectoriel de tout ministère.

Ainsi, les commissariats et sous-commissariats sont en fait les différents ministères et secrétariats actuels et ils ont, pour la plupart, des ramifications dans les régions et les secteurs, les délégations régionales et sectorielles. Bolama, chef-lieu de la région BolamaBijagos et du secteur de Bolama (Bolama, São-João et Gallinhas), a donc des représentants des délégations régionales et des délégations sectorielles. Les délégations régionales ont un rôle de coordination et d'encadrement de leurs délégations sectorielles, en plus d'assurer le lien entre celles-ci, elles-mêmes et Bissau. Les différentes délégations régionales à Bolama, ont généralement sous leur responsabilité, quatre délégations sectorielles qui correspondent chacune à un secteur de la région. A Bolama, on remarque la présence de huit délégations représentant les ministères suivants:

- Ministère du Développement Rural;

1 Republica da Guiné-Bissau, Boletim Oficial, No. 1, 4 de janeiro de 1975, No. 28, 12 de julho de 1975, No. 27, 11 de julho de 1986. 
- Ministère du Plan;

- Ministère de l'Education;

- Ministère des Finances;

- Ministère du Commerce (Armazens do Povo);

- Ministère des Forêts;

- Ministère de la Santé Publique; et,

- Ministère des Ressources Naturelles.

\subsubsection{Le pouvoir national}

L'organe suprême du pouvoir d'Etat est l'Assemblée Nationale Populaire (A.N.P.). ${ }^{1}$ Nommée par les députés des conseils régionaux pour une période de quatre ans, l'Assemblée Nationale Populaire approuve les lois et les résolutions, "délibère et décide des questions fondamentales touchant la politique intérieure et extérieure de l'Etat, et contrôle l'application de la ligne politique et économique décidée par le P.A.I.G.C.". ${ }^{2}$ Elle est dirigée par un président. ${ }^{3}$

Le Conseil d'Etat, élu pour quatre ans par les députés de l'A.N.P., voit ses attributions s'appliquer tant aux questions d'ordre interne qu'aux politiques internationales et son influence est prépondérante. En effet, son président, élu en son sein, représente dans les relations internationales l'Etat bissau-guinéen, dont il est le Président. ${ }^{4}$ Il est de plus le

\footnotetext{
1 Paulette Pierson-Mathy, op.cit, page 134.

2 Ibidem.

3 Groupement Lavalin/Delcanda, Etude générale sur les transports. s.1., Banque Africaine de Développement, juin 1980, volume I, Base socio-économique, page 2-6.

4 Ibidem.
} 
commandant suprême des F.A.R.P. (Forces Armées Révolutionnaires du Peuple), ${ }^{1}$ ces F.A.R.P. dont Carlos Lopes dit d'elles:

"Le centre de gravité du pouvoir politique en Guinée-Bissau pèse sans aucun doute sur les FARP. Pour aboutir, toute stratégie politique doit trouver un appui tactique au sein des FARP. Il faut souligner que, pour suivre la terminologie de J. Nyerere, les FARP ne sont pas des mercenaires. Au contraire, il s'agit de la force politique la plus militante et la mieux organisée du PAIGC. Les soldats sont les plus conscients des enjeux politiques et idéologiques et ils constituent le seul groupe totalement alphabétisé (Le succès de l'alphabétisation au sein des FARP est dû à leur discipline car les autres campagnes d'alphabétisation furent des échecs). Le capital d'organisation et d'expériences collectives au sein des FARP en fait l'une des forces de défense et de réalisation des acquis de la lutte de libération nationale". 2

Enfin, le Conseil des Commissaires d'Etat, actuel Conseil des Ministres, ${ }^{3}$ base son activité sur les lois et les résolutions de l'A.N.P. ainsi que sur les décisions du Conseil d'Etat. Ses attributions consistent à veiller à la réalisation du programme politique, économique, social et culturel de l'Etat, en plus de la défense et de la sécurité nationale. Il est en outre responsable de le coordination, du contrôle et de la direction des activités des commissariats et des secrétariats d'Etat, ainsi que des comités d'Etat régionaux et sectoriels. ${ }^{4}$

1 Paulette Pierson-Mathy, op, cit., page 138.

2 Carlos Lopes, Ethnie, Etat et rapports de pouvoir en Guinée-Bissau, op. cit., page 59.

3 Lars Rudebeck, "Problème de pouvoir populaire et de développement", Research Report, No. 63, 1982, chapitre IV, Le coup d'Etat militaire du 14 novembre 1980.

4 Paulette Pierson-Mathy, op.cit, page 139. 


\subsubsection{Les provinces}

Le décret ministériel numéro 3/87 de mai 1987,1 consacre les fonctions d'une nouvelle entité administrative supra-régionale, la province, dont l'existence était déjà un fait accompli. Au nombre de trois (Provinces du Sud, du Nord et de l'Est), les provinces s'avèrent être un nouvel intermédiaire administratif entre les régions et le centre, chacune étant sous l'autorité d'un ministre dénommé ministre résident. En ayant à s'impliquer activement dans le développement des régions qui lui sont assignées, chaque cabinet provincial doit veiller à l'élaboration et au suivi de projets de développement dans leur province. ${ }^{2}$ De même il se doit de participer au processus national de préparation et d'exécution des plans nationaux de développement. L'implication des cabinets provinciaux dans le Deuxième Plan Quadriennal de Développement pendant les années 1986 à 1988, les auront confirmé dans leurs nouvelles fonctions auxquelles elles ont impliqué les comités d'Etat régionaux de leur province. Pour l'instant, les cabinets des ministres résidents semblent dominer sur les comités d'Etat régionaux de leur province avec qui ils collaborent et grâce auxquels ils établissent peu à peu une relation administrative avec les organes régionaux qui dépendaient jusque là des comités. On peut croire, de l'avis de plusieurs, que les cabinets des ministres résidents remplaceront progressivement les comités d'Etat régionaux. ${ }^{3}$

\footnotetext{
1 Republica da Guiné-Bissau, Boletim Oficial, No. 13, 4 de maio de 1987.

2 Ibidem.

3 Entrevue avec Alfredo Medina, Secrétaire du Comité d'Etat Régional, Bolama, février 1988.
} 


\section{TABLEAU 4.2}

Province du Sud

Division administrative

1987

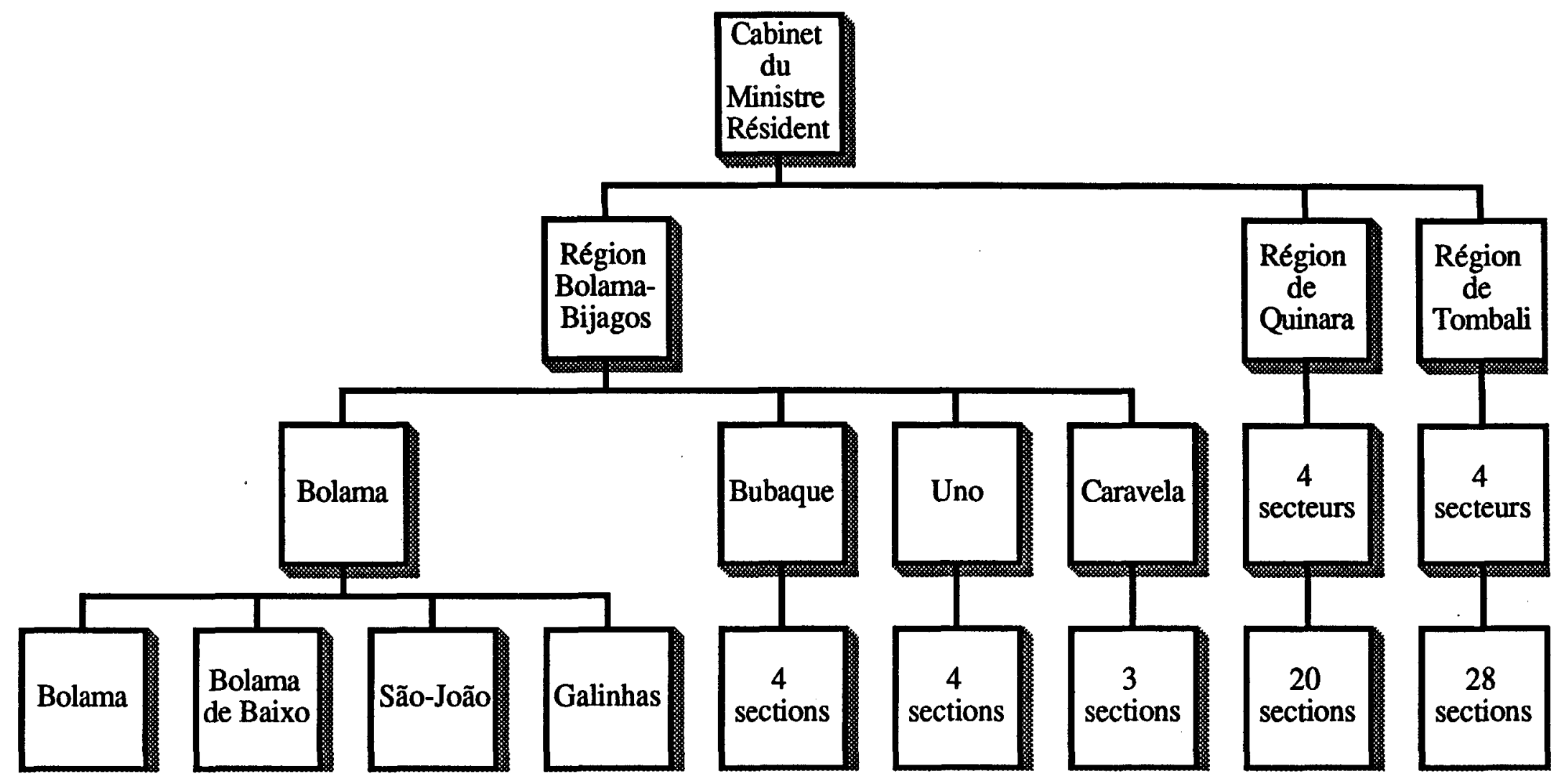

Source: D.P.R., Lista nominal das secções por sectores administrativos, Bissau, 25 de fevereiro de 1987;

Carlos Lopes, "Ethnie, Etat et rapports de pouvoir en Guinée-Bissau", Itinéraires, notes et travaux, No. 22, mars 1982, Première Edition, Genève, page 58. 
Cette restructuration qui affecte les régions et qui, vraisemblablement, fera disparaître les comités d'Etat régionaux, peut provenir d'un désir de rationalisation des dépenses étatiques de même que d'une recherche d'un meilleur encadrement dans l'exécution des tâches administratives. Comme nous le remarquerons à quelques reprises, l'efficacité et la compétence des membres des comités d'Etat régionaux dans les tâches administratives plus complexes qui leur reviennent, telles que celles liées au développement de leur région, sont souvent des plus douteuses. Une centralisation au niveau des provinces, dans l'éventualité d'un encadrement efficace de celles-ci, pourrait avoir un effet bénéfique en ce sens. Nous ignorons cependant si les actuelles structures ministérielles régionales et sectorielles continueront d'agir comme elles le font encore, mais la disparition des comités d'Etat régionaux, qui ne concerne d'ailleurs pas les comités sectoriels, peut laisser croire que les délégations régionales seront abolies, contrairement aux délégations sectorielles qui se retrouveraient sous l'autorité de la province. Une telle centralisation peut, par contre, nous faire craindre que les administrations provinciales n'héritent aussi des problèmes qui affectaient jusque là les comités d'Etat régionaux dont principalement ceux liés à la logistique et à la communication avec l'ensemble de leur territoire. Dans ces cas précis, on peut même s'inquiéter de voir les provinces hériter de territoires encore plus étendus que ceux qu'avaient leurs prédécesseurs.

Cette tendance à l'abolition des comités d'Etat régionaux est facilement perceptible dans la création de chambres municipales dans les sièges des provinces et des régions qui, aux fonctions directement reliées à l'administration municipale (levée des impôts, budgétisation, ordre public, entretien, etc.) se verraient accordées celles du développement de la ville. ${ }^{1}$ Toutes ces tâches relevaient jusqu'ici en grande partie des comités d'Etat régionaux.

1 Gabinete do Ministro Residente, Projecto de Câmara Municipal. Bolama, Bolama, Provincia do Sul, março 1988, page 3. 
Cette centralisation administrative ne semble pas devoir déborder ce cadre pour affecter, du moins à court terme, l'organisation politique. Le lien très étroit que nous avons constaté, tout au long de ce chapitre, entre le Parti et l'Etat, gardera donc toujours son caractère particulier où apparemment ces deux entités sont séparées, du moins au niveau des structures. En fait, le P.A.I.G.C., à travers une pratique qui ne peut que nous rappeler l'importante école léniniste de l'infiltration des appareils d'Etat, a su soumettre les organes publics à ses vues, sans qu'officiellement, administration et politique ne soient liées et que, comme le souligne Lopes, il ne soit taxé de "Parti-Etat". ${ }^{1}$ Les liens très étroits entre le Parti et l'Etat s'expliquent aisément par la présence des mêmes personnes aux postes dirigeants du Parti et de l'Etat. ${ }^{2}$ D'une façon plus spécifique, et pour illustrer cette affirmation, la composition du Cabinet du Ministre Résident de la Province du Sud confirme cette constatation et il est d'un certain intérêt de prendre connaissance de l'énumération des principales fonctions que cumulent les membres de ce cabinet: ${ }^{3}$

- Président du Cabinet: membre du Comité Central du P.A.I.G.C. et Ministre Résident;

- Premier Vice-Président: membre du Comité Central du P.A.I.G.C. et Président du Comité d'Etat Régional de BolamaBijagos;

- Deuxième Vice-Président: membre du Comité Central du P.A.I.G.C. et Président du Comité d'Etat Régional de Quinara;

1 Carlos Lopes, A transição histórica na Guiné-Bissau: de movimento de libertação nacional ao Estado op. cit., page 169.

2 Paulette Pierson-Mathy, op_cit, page 140.

3 Província do Sul, Acta: primeira reunião do Conselho Directivo Provincial Alargado aos responsaveis regionais, Bolama, novembro de 1986, page 1. 
- Troisième Vice-Président: membre du Comité Central du P.A.I.G.C. et Président du Comité d'Etat Régional de Tombali; et,

- Secrétaire: membre du Comité Central du P.A.I.G.C. et Secretaire de l'Organisation du Parti de Tombali.

Il nous semble que le refus que les dirigeants bissau-guinéens opèrent face à l'identification de leur Parti au marxisme-léninisme relève de deux optiques bien différentes. La première, que l'on pourrait associer au leader idéologique du P.A.I.G.C., Amilcar Cabral, consiste à refuser d'identifier le mouvement bissau-guinéen à ce mode de pensée pour de pures raisons philosophiques. D'après lui, le marxisme-léninisme et plus généralement le socialisme, ne correspondent pas à la réalité du pays. La seconde tendance, celle des dirigeants actuels et nationaux, n'est peut-être que stratégique, afin de ne pas se mettre à dos les alliés occidentaux qui sont nécessaires au financement du développement de la nation, et en plus de conserver une ligne internationale officielle de non-alignement. Le P.A.I.G.C. nous semble effectivement grandement inspiré du modèle léniniste du parti, la conscience éclairée ${ }^{1}$ de la classe révolutionnaire, son détachement d'avant-garde, ${ }^{2}$ son détachement organisé, ${ }^{3}$ sa forme suprême d'organisation ${ }^{4}$ devant mener le peuple à une société juste et équitable. ${ }^{5} \mathrm{Au}$ niveau de son organisation même, le P.A.I.G.C. démontre une très nette propension à l'infiltration des divers paliers de l'organisation sociale bissau-guinéenne, exception faite des structures traditionnelles qu'il tente cependant de contourner par les

1 Lénine, Que faire?, s.l., Editions du Seuil, 1966, page 135.

2 J. Staline, Des principes du léninisme: conférences faites à l'Université Sverdlov Pékin, Editions en Langues Etrangères, 1970, page 104.

3 Ibid., page 107.

4 Ibid., page 110.

5 P.A.I.G.C., Teses para o IV Congresse de PAIGC, op. cit., page 7. 
comités de tabanca et la création d'une unité nationale basée sur la négation des particularités ethniques. Lénine lui-même tire d'ailleurs comme enseignement de son expérience révolutionnaire:

"De là deux conclusions pratiques d'une grande importance: la première, c'est que la classe révolutionnaire, pour remplir sa tâche, doit savoir prendre possession de toutes les formes et de tous les côtés, sans la moindre exception, de l'activité sociale (quitte à compléter, après la conquête du pouvoir politique et parfois au prix d'un grand risque et d'un danger énorme, ce qu'elle n'aura pas terminé avant cette conquête); la seconde, c'est que la classe révolutionnaire doit se tenir prête à remplacer vite et brusquement une forme par une autre." 1

1 Lénine, La maladie infantile du communisme (le "gauchisme"), Pékin, Editions en Langues Etrangères, 1970, page 99. 


\section{CHAPITRE CINQ}

\section{LE MODELE DE DEVELOPPEMENT BISSAU-GUINEEN}

5.1 Planification et plans quadriennaux

Les premières années d'indépendance de la Guinée-Bissau, celles précédant le "Mouvement de réajustement du 14 novembre" 1 (1974-1980), furent marquées par la nécessité de procéder à l'adaptation d'un appareil d'Etat forgé durant la lutte armée selon les principes du P.A.I.G.C., aux réalités de l'héritage colonial. 2

Le III' Congrès du P.A.I.G.C. de 1977 établit pour une première fois, la stratégie de développement économique et social du pays, sur les bases des principes politiques du Parti, centralisme démocratique et direction collective, ${ }^{3}$ orientant l'économie nationale vers l'indépendance, la planification et l'harmonie. En fait, le $\mathrm{III}^{\circ}$ Congrès insiste sur la nécessité d'accorder à l'agriculture un rôle fondamental puisque l'on identifie ce secteur à l'activité de base de la population, seule activité permettant d'ailleurs une accumulation de la richesse nationale rapide et efficace. Ce Congrès souligne l'importance de procéder à un

\footnotetext{
1 République de la Guinée-Bissau, Texte présenté par la République de la Guinée-Bissau à la Conférence des Pays les Moins Avancés, s.l.n.d., page 23.

2 Guinée-Bissau, Conférence des Organisations Non Gouvernementales: document d'orientation, Bissau, 7-10 novembre 1985, page 15.

3 Ibid., page 16.
} 
développement intégré où agriculture, industrie et secteur tertiaire uniront leurs efforts pour que l'industrie puisse jouer un rôle moteur et catalyseur dans le développement du secteur primaire, en accord avec le principe de la recherche de la maximalisation et de la valorisation des ressources naturelles et humaines du pays au profit du peuple. ${ }^{1}$

Toutefois, la ligne édictée par le Parti ne fut pas suivie et, plutôt que d'accorder la priorité à l'agriculture, les dirigeants de l'époque privilégièrent le secteur industriel selon un développement irréaliste où l'on concentra dans la capitale des projets de trop grande envergure par rapport aux capacités réelles du pays comme de ses besoins: technologie trop poussée, manque de ressources financières, incapacité de gestion et d'entretien.2 Parallèlement à cela, les entreprises commerciales d'Etat ont adopté des comportements contraires aux intérêts des paysans producteurs en ignorant complètement toute mesure propre à stimuler la production ou à approvisionner les campagnes par la distribution et la vente, tant des biens de consommation et de production que de la production rurale ellemême. Ceci ne fit que replier de plus en plus le monde rural sur lui-même, dans ses systèmes traditionnels d'autoconsommation. ${ }^{3}$

Toutes ces attitudes de la part des autorités, combinées à l'irrégularité climatique qui affecta le pays durant cette période et à la conjoncture internationale défavorable aux pays du tiers-monde, furent interprétées, après le coup d'Etat de 1980, comme responsables de la grave crise économique et financière qui frappa le pays. Les conséquences les plus palpables de cette crise se traduisirent par l'épuisement des réserves en devises étrangères, une inflation élevée, la désorganisation des circuits internes de commercialisation et de distribution, et la

\footnotetext{
1 Ibid., page 18.

2 Ibid., page 19.

3 République de la Guinée-Bissau, op.cit., pages 23-24.
} 
baisse de la production agricole. ${ }^{1}$ L'explication habituelle que l'on attribue à ce phénomène de déviation par rapport aux politiques du Parti, incombe à l'héritage du passé:

"Les causes de cette déviation sont nombreuses mais, en ce qui concerne la politique économique, elles peuvent être résumées ainsi: l'immaturité de l'appareil d'Etat et l'acceptation de bon nombre de structures administratives héritées du colonialisme ont entraîné une incapacité réelle au niveau de la direction de l'activité économique et une absence presque totale de coordination centrale des décisions prises dans le domaine de la politique économique. Chaque ministère, chaque département d'Etat a pu agir d'une façon relativement indépendante par rapport aux autres, surtout en ce qui concerne l'exécution de projets d'investissement." 2

Le gouvernement mis en place à partir de 1980, s'est efforcé de baser ses opérations de développement en conformité avec les vues du Parti. Pour ce faire, il fut décidé d'adopter une stratégie de développement à court et à moyen terme. A court terme, il s'agissait de régulariser la conjoncture économique du pays et d'assurer les conditions nécessaires au démarrage du processus de développement prévu par le P.A.I.G.C. et ce, afin de pouvoir, à moyen terme, le mettre en branle. Le pays initia ainsi un programme économique biennal pour les années 1981 et 1982. Ses objectifs principaux étaient de diminuer le déficit courant des finances publiques, de réviser le système de change en vigueur, de réorganiser les entreprises d'Etat spécialisées dans le commerce en remettant la distribution au détail au commerce privé, de redéfinir la politique des prix pratiqués aux producteurs afin de stimuler la production agricole, et d'initier un mécanisme central de contrôle des importations dans le cadre des priorités définies en tenant compte du budget du pays. ${ }^{3}$

Ce programme, qui ne constituait en fait qu'une étape de prise en main, par la nouvelle administration, d'une économie au bord du gouffre, trouve sa justification dans le rôle de passage qu'il sait se donner, entre une administration anarchique du développement et la mise

1 Ibid., page 24.

2 République de la Guinée-Bissau, op.cit., page 23.

3 Ibid., pages 24-25. 
en place et la préparation d'un moyen rigide d'exécution des objectifs nationaux en matière de développement économique et social. Ainsi, ce programme a-t-il vu le gouvernement adopter, en 1982, par la Conseil de la Révolution, la planification comme pierre angulaire de la construction d'une économie nationale indépendante, et ainsi pousser les différents organes et acteurs du pays, à conjuguer leurs efforts pour l'élaboration et la réalisation du Premier Plan Quadriennal de Développement Economique et Social (1983-1986). ${ }^{1}$ Dès lors, les bases sont jetées pour le fonctionnement d'une économie planifiée sous l'égide du Parti comme en fait foi l'introduction au Premier Plan Quadriennal:

"Os congressos sucessivos du Partido indicaram claramente que o nosso desenvolvimento económico é planificado e que a direç̧ão planificada da economia é regida pelo principio do centralismo democrático. Por consequinte, o Plano só pode ser imperativo: isto significa que os diferentes Ministérios e organismos públicos, as empresas públicas e mistas devem orientar as suas acções no sentido da realização do Plano e prestar contas dos esforços efectuados e dos seus resultados." 2

\subsubsection{Le Premier Plan Quadriennal de Développement Economique et Social,} $1983-1986$

Pour faire suite aux efforts de relance économique entrepris lors du programme biennal de 1981-1982 et assurer ainsi les conditions minimales nécessaires à l'exécution du Premier Plan Quadriennal, d'importantes mesures d'assainissement furent entreprises lors de la

1 Republica da Guiné-Bissau, Boletim Oficial, No. 6, 6 de fevereiro de 1982.

2 S.E.P.C.I., Primeiro Plano Quadrienal de Desenvolvimento Economico e Social 1983-1986, Bissau, s.d., Volume 1. Relatorio Geral, page 1;

"Les congrès successifs du Parti indiquent clairement que notre développement économique est planifié et que la direction planifiée de l'économie est régie par le principe du centralisme démocratique. Par conséquence, seul le Plan peut être impératif: ceci signifie que les différents Ministères et organismes publics, les entreprises publiques et mixtes doivent orienter leurs actions dans le sens de la réalisation du Plan et rendre compte des efforts effectués et de leurs résultats" (traduction de l'auteur). 
première phase du plan (1983-1984). Connues sous le nom de Programme de Stabilisation Economique et Financière (P.E.E.F.), ces mesures s'appuient sur quatre axes principaux: ${ }^{1}$

- assainissement de la situation économique et financière interne et externe;

- réorganisation des circuits économiques et commerciaux;

- utilisation judicieuse de l'aide externe; et,

- contrôle de l'économie.

Le P.E.E.F. s'est traduit concrètement par la libéralisation du commerce. Les mesures de dévaluation de la monnaie ont aussi eu des effets très probants au niveau interne, tout comme l'encouragement au recours à l'aide externe qui, au plan régional, s'est traduit par l'apparition de nombreux projets de coopération. Il est évident que toutes ces mesures eurent leur contrepartie sur le plan macro-économique national, mais leur étude déborderait largement le cadre régional que nous avons fixé à notre mémoire.

Le Plan Quadriennal en tant que tel poursuit des objectifs visant à contrer le plus possible le déficit extérieur, à satisfaire les besoins essentiels de la population, notamment en parvenant à l'auto-suffisance alimentaire en ce qui a trait aux produits de base, et à mettre sur pied un développement économique et social équilibré.

Pour ce faire, le Plan a été divisé en deux parties. Les deux premières années forment un tronc commun où l'on recherche l'implantation et la poursuite du programme de stabilisation. A partir de 1985, on adopte deux scénarios de financement et de réalisation des activités, une variante supérieure et une variante inférieure. La variante inférieure repose sur l'incertitude des autorités gouvernementales face entre autres, à la réaction de l'économie du pays par rapport à l'implantation du Programme de Stabilisation, à l'appui qu'accordera la

1 Guinée-Bissau, op.cit., pages 23-24.

2 République de la Guinée-Bissau, op. cit., page 27. 
communauté internationale en terme de financement et d'assistance technique à la réalisation du Plan, ${ }^{1}$ et à la fragilité des appareils économiques d'Etat qui pourraient ne pas être en mesure de réaliser entièrement les prévisions. ${ }^{2}$

Le Plan s'articule autour de quatre axes: un Bloc Prioritaire, un Bloc d'Appui Principal, un axe des Secteurs Sociaux et enfin un axe composé des Autres Secteurs. ${ }^{3}$

Le Bloc Prioritaire regroupe les secteurs de l'agriculture, des pêches, des forêts et de la transformation du bois, et des mines.

Le Bloc d'Appui Principal comprend les secteurs des transports, du commerce, des postes et télécommunications, de l'hydraulique et de l'administration. Son rôle, tout aussi trivial que cela puisse paraître, est d'appuyer ou de compléter les secteurs du Bloc Prioritaire. 4

Le groupe des Secteurs Sociaux est composé de la santé, de l'urbanisme, de l'hygiène et l'habitation, de l'information et de la culture, de l'éducation, de la jeunesse et des Anciens Combattants.

En ce qui a trair aux Autres Secteurs, notons qu'ils incluent l'industrie, l'énergie, la construction, les travaux publics et le tourisme.

$\overline{1}$ Lors de la publication du Plan, en 1983, le financement pour la variante supérieure n'était assuré qu'à 60,5\%, soit $6 \%$ par la Guinée-bissau et $54,5 \%$ par le financement externe (S.E.P.C.I., op.cit. page 91). Les dépenses d'investissement nécessaires à la réalisation de la variante supérieure étaient estimées à 16424,4 millions de P.G. (soit environ $400 \$$ millions américains en prix constants de 1983); celles de la variante inférieure se chiffraient à 25\% plus bas, soit à environ 12318,3 millions de P.G., environ $300 \$$ millions américains (Ministério du Plano, Quarta reunião do Conselho Consultivo do Ministério de Plano: primeira fase do II Plano Ouadrienal, Republica da Guiné-Bissau, fevereiro de 1988, pages 8-9).

2 S.E.P.C.I., op cit., pages 6-7.

3 Ibid, page 91.

4 Bernardino Cardoso, "Mémorandum à l'intention de l'O.I.T. (Organisation Internationale du Travail)", cité dans, Philippe de Braconier, Elizabeth Drory, Rapport de mission de juin 1985: préétude de l'Tle de Paix de Bolama, Huy, Iles de Paix, 1985, Annexe. 
Cette répartition des différents axes correspond à la variante supérieure et se distribue de la façon présentée au Tableau 5.1. La variante inférieure, pour sa part, est formée à partir d'un "noyau central" regroupant le Bloc Prioritaire et le Bloc d'Appui Principal.1

Quant à la part des régions dans ce Plan, nous verrons qu'elle se limite à l'exécution des projets de développement régionaux tels qu'identifiés par les autorités régionales compétentes soit, essentiellement, les Cabinets Régionaux de Planification.

Tel que présenté dans le rapport général du Ministère du Plan, ${ }^{2}$ le Premier Plan Quadriennal du pays apparaît comme un document ne faisant état que de la situation actuelle des différents secteurs de l'économie bissau-guinéenne en fonction de la place qu'ils occupent au sein des quatre axes, et des politiques à entreprendre pour l'atteinte les objectifs sectoriels poursuivis. En aucun cas, ces démonstrations ne font référence aux étapes exactes, en termes d'investissements précis ou de mesures concrètes applicables à court terme par les différents ministères concernés. Ainsi, les objectifs sont formulés pour chacun des secteurs, les budgets sont distribués par région ou, plus exactement, par zone. L'aspect pratique de la gestion fait par contre admirablement défaut et la mise en opération du Plan revêtira toujours estime-t-on un défi de taille pour les membres des organes concernés, ministères, secrétariats et organismes, dont le manque de formation et d'encadrement du personnel et ce, à leurs différents paliers, s'avéreront un obstacle majeur à la concrétisation des objectifs du Plan.

\footnotetext{
1 S.E.P.C.I., op. cit., page 92.

2 Ibid, 205 pages.
} 
TABLEAU 5.1

Premier Plan Quadriennal dépenses de développement 1983-1986

(en millions de PG)

\begin{tabular}{|l|r|r|r|r|r|r|r|r|r|r|}
\hline \multirow{2}{*}{ Axe } & \multicolumn{2}{|c|}{1983} & \multicolumn{2}{|c|}{1984} & \multicolumn{2}{c|}{1985} & \multicolumn{2}{c|}{1986} & \multicolumn{2}{c|}{ Somme totale } \\
\cline { 2 - 11 } & Montant & $\%$ & Montant & $\%$ & Montant & $\%$ & Montant & $\%$ & Montant & $\%$ \\
\hline Bloc Prioritaire & 776,1 & 36,4 & 1281,2 & 33 & 1202,2 & 22,2 & 1208,4 & 24,2 & 4467,9 & 27,2 \\
Bloc d'Appui Principal & 659,0 & 30,9 & 803,1 & 20,7 & 1291,3 & 23,8 & 947,9 & 19,0 & 3701,3 & 22,5 \\
Secteurs Sociaux & 303,9 & 14,3 & 534,7 & 13,8 & 1073,7 & 19,8 & 916,5 & 18,4 & 2828,8 & 17,2 \\
Autres Secteurs & 390,8 & 18,4 & 1263,5 & 32,5 & 1853,6 & 34,2 & 1918,5 & 38,4 & 5426,4 & 33,1 \\
\hline \multicolumn{1}{|c|}{ Total } & 2129,8 & 100 & 3882,5 & 100 & 5420,8 & 100 & 4991,3 & 100 & 16424,4 & 100 \\
\hline
\end{tabular}

Source: S.E.P.C.I., Primeiro Plano Quadrienal de Desenvolvimento Economico e Social 1983-1986. Volume 1. Relatorio Geral, Bissau, page 91. 
L'analyse du secteur du développement rural qui, comme on le sait, constitue en fait le secteur privilégié en matière de développement dans le discours officiel, est assez éloquente à cet égard. ${ }^{1}$ On procède tout d'abord dans le Plan, au constat de la situation actuelle en soulignant limportance de ce secteur qui occupe la très grande majorité de la population du pays. Vient ensuite l'énumération des principaux facteurs limitatifs, tels l'approvisionnement insuffisant en biens de consommation et l'importante émigration vers l'étranger. Le survol des contraintes du secteur agricole est suivi d'un aperçu de ses potentialités dont l'abondance de terres arables et les atouts climatiques. On procède alors à l'établissement des orientations de base et à l'énumération des actions à entreprendre pour leur réalisation. Ainsi apprend-t-on que pour atteindre l'autosuffisance alimentaire, limiter l'exode rural et éviter les déséquilibres régionaux, le pays devra, d'une part, assurer un approvisionnement suffisant des campagnes en moyens de production, d'autre part encourager la céréaliculture et l'élevage grâce à un programme de multiplication de semences améliorées et de "développement de la production bovine dans un esprit d'intégration agriculture-élevage" 2 et enfin, appuyer les institutions agricoles gouvernementales dans leurs programmes de formation et de planification. Le plan de développement du secteur agricole se termine par une liste des principaux projets à entreprendre dont un recensement agricole, l'amélioration des rizières et la réalisation de projets agricoles importants. Le tout se termine par des prévisions concrètes en matière de production agricole pour les principaux produits pour la fin de la période que couvre le Plan.

Un document plus précis, préparé par un groupe de travail sur l'agriculture, l'élevage et les forêts, ${ }^{3}$ pousse plus à fond cette analyse et énonce certaines politiques précises mais tout

1 Ibid., pages 95-99.

2 Ibid, page 97.

3 MICEPCI. Primeiro Plano Quadrienal de Desenvolvimento Económico e Social 1983-1986. Bissau, Grupo de Trabalho Sectorial (Agricultura, Pecúaria e Florestas), abril de 1982. A: Relatório da situação actual, pages 47-104. 
aussi théoriques. L'atteinte de l'autosuffisance alimentaire du pays avant les années 1990 en matière de riz ${ }^{1}$ et, d'une manière plus générale, le développement harmonieux du secteur agricole, doivent passer par l'organisation du monde rural. Aussi veut-on développer et mobiliser la paysannerie en lui fournissant l'opportunité de s'unir en organisations, associations ou coopératives de producteurs. ${ }^{2} \mathrm{Ce}$ premier pas vers une organisation des paysans est original et vise certes à remettre à la base une tâche que les appareils gouvernementaux n'ont pas jusqu'ici réussi à réaliser, soit une véritable relance de la production agricole. Le Ministère du Développement Rural n'aurait, dans cette optique, qu'un rôle de formation et d'encadrement logistique du producteur agricole et devrait concentrer ses activités dans la mobilisation des masses rurales, par l'intermédiaire des comités de tabanca, en vue de la prise en charge par les paysans de leur propre développement et la mise en place d'embryons de futures cooperatives. ${ }^{3}$

Pendant la période du Plan, pour la région Bolama-Bijagos, on ne constate aucune forme de mobilisation rurale de la part du Ministère du Développement Rural. Nous pouvons vraisemblablement attribuer cet état de fait aussi bien à l'absence d'efforts concrets de la part du ministère qui ne semble pas disposer d'une politique bien établie en la matière, qu'aux réticences évidentes qu'émettent les villageois face aux autorités gouvernementales.

En ce qui concerne les autres secteurs du Bloc Prioritaire, les actions proposées par le Plan sont sensiblement de même nature et se caractérisent toutes par les besoins de renforcement des structures déjà existantes, et par ceux de formation du personnel de l'Etat. Enfin, on remarque la nécessité de voir se développer un système de commercialisation plus

\footnotetext{
1 Ibid., page 56.

2 Ibid., page 50.

3 Ibid., page 63.
} 
adéquat assurant l'approvisionnement de la population du pays en moyens de production nécessaires, parallèlement à l'assurance d'un écoulement efficace de la production. ${ }^{1}$

Le Bloc d'Appui Principal 2 qui, comme son nom l'indique, doit veiller à fournir l'encadrement logistique nécessaire au développement du pays, a besoin d'un renforcement et d'une amélioration de son réseau (hydraulique, transport, télécommunications), de même que d'une formation plus adéquate de sa main-d'oeuvre. Au niveau de l'administration publique, aux besoins de formation du personnel s'ajouteront des efforts de rationalisation des dépenses et d'amélioration de la productivité. Enfin, le secteur du commerce devra, parallèlement à sa libéralisation, veiller à développer son réseau de distribution, conjointement à la création d'entreprises de transport à l'échelle du pays.

Le bloc des Secteurs Sociaux, ${ }^{3}$ quant à lui, devra porter ses efforts sur la décentralisation de ses services, la construction de nouvelles unités et la formation de son personnel.

Quant aux Autres Secteưrs, ${ }^{4}$ ils présentent tous les mêmes besoins que les trois axes précédents. La formation, le développement de leurs réseaux et l'amélioration des services de distribution et d'écoulement constituent les grandes lignes que leur dresse le Plan.

\footnotetext{
1 S.E.P.C.I.., 0D. cit., pages 104-117.

2 Ibid, pages 119-136.

3 Ibid, pages 139-161.

4 Ibid., pages 165-181.
} 


\section{TABLEAU 5.2}

Distribution des investissements

Premier Plan Quadriennal

Région Bolama-Bijagos et ensemble du pays

1983-1986

(en millions de P.G.)

\begin{tabular}{|c|c|c|c|c|c|c|}
\hline \multirow{2}{*}{ Secteur } & \multicolumn{2}{|c|}{ Région Bolama-Bijagos } & \multicolumn{2}{|c|}{ Administration nationale } & \multicolumn{2}{|c|}{ Total Guinée-Bissau } \\
\hline & Montant & $\%$ & Montant & $\%$ & Montant & $\%$ \\
\hline $\begin{array}{l}\text { Développement rural } \\
\text { Forêt } \\
\text { PÉche } \\
\text { Hydraulique } \\
\text { Mines } \\
\text { Transports } \\
\text { Telecommunications } \\
\text { Commerce } \\
\text { Construction } \\
\text { Energie } \\
\text { Industrie } \\
\text { Tourisme } \\
\text { Education } \\
\text { Santé } \\
\text { Jeunesse et Sport } \\
\text { Information et Culture } \\
\text { Combattants de la Patrie } \\
\text { Administration Publique } \\
\text { Projets régionaux }\end{array}$ & $\begin{array}{r}15,6 \\
1,3 \\
111,9 \\
- \\
- \\
7,6 \\
- \\
- \\
6,0 \\
7,4 \\
-\overline{1} \\
28,0 \\
-\overline{1} \\
21,6 \\
- \\
- \\
- \\
4,6\end{array}$ & $\begin{array}{r}0,5 \\
0,2 \\
15,2 \\
- \\
- \\
0,3 \\
- \\
- \\
0,1 \\
1,5 \\
- \\
18 \\
- \\
2 \\
- \\
- \\
- \\
- \\
2\end{array}$ & $\begin{array}{r}867,5 \\
74,2 \\
442,8 \\
178,1 \\
222,8 \\
525,4 \\
242,0 \\
79,2 \\
1425,1 \\
410,2 \\
582,2 \\
- \\
469,0 \\
365,4 \\
32,4 \\
100,00 \\
34,0 \\
486,7 \\
120,0\end{array}$ & $\begin{array}{r}29,5 \\
13,4 \\
60,3 \\
30 \\
87 \\
23 \\
93 \\
100 \\
36 \\
81 \\
56 \\
- \\
67 \\
37 \\
7 \\
100,00 \\
62 \\
100 \\
41\end{array}$ & $\begin{array}{r}2920,4 \\
555,6 \\
735,1 \\
598,0 \\
256,8 \\
2277,4 \\
260 \\
79,2 \\
3992,4 \\
506,1 \\
1037,2 \\
152,6 \\
698,5 \\
991,6 \\
432,4 \\
100,00 \\
54,4 \\
486,7 \\
290,0\end{array}$ & $\begin{array}{l}100 \\
100 \\
100 \\
100 \\
100 \\
100 \\
100 \\
100 \\
100 \\
100 \\
100 \\
100 \\
100 \\
100 \\
100 \\
100 \\
100 \\
100 \\
100\end{array}$ \\
\hline Total & 204,0 & 1 & 6657,0 & 40 & 16424,4 & 100 \\
\hline
\end{tabular}

Source: S.E.P.C.I., Primeiro Plano Quadrienal de Desenvolvimento Economico e Social 1983-1986. Volume 1, Relatorio Geral, Bissau, page 201. 
La place que l'on accorde au développement régional est somme toute relative malgré l'existence de structures de planification régionale, les Cabinets Régionaux de Planification. Chaque ministère ou organe en région, se voit attribuer, par les autorités concernées, les fonds nécessaires à la réalisation du plan national sur son territoire. ${ }^{1}$ La région BolamaBijagos, qui représente $3 \%$ de la population du pays, se voit ainsi octroyer $1 \%$ du total national consacré au Plan (voir Tableau 5.2), soit 204000 contos (milliers de pesos), ce qui représente $0,5 \%$ du total national consacré au développement rural, $0,2 \%$ du total du domaine des forêts, $15,2 \%$ des pêches, $1,5 \%$ de l'énergie et $2 \%$ de la santé, 2 distribués tel qu'indiqué au Tableau 5.3.

Le domaine de la pêche compte pour la majeure partie des investissement régionaux. L'intervention du Secrétariat d'Etat aux Pêches dans le projet Pescarte à Bolama, est la concrétisation, à ce titre, des politiques du Plan sur le développement de la pêche. ${ }^{3}$

Le tourisme qui, des dires mêmes des responsables du Plan, n'est pas une priorité, puisqu'il est classé dans les Autres Secteurs, occupe cependant le deuxième rang du budget régional avec le projet de préparation d'un plan de développement balnéaire sur l'île de Caravela et l'amélioration du réseau hôtelier national, ${ }^{4}$ ce qui touche directement la station balnéaire de Bubaque.

La santé vient au troisième rang, ce qui peut s'expliquer par les désirs de décentralisation des services de santé dont fait état le Plan.5

\footnotetext{
1 Ibid, page 93.

2 Ibid., page 203.

3 Ibid, pages 110-111.

4 Ibjd, pages 180-181.

5 Ibid, pages 139-140.
} 
TABLEAU 5.3

Répartition des sommes consacrées au

Premier Plan Quadriennal

Région Bolama-Bijagos

1983-1986

(en millions de contos)

\begin{tabular}{|l|r|r|}
\hline \multicolumn{1}{|c|}{ Secteur } & \multicolumn{1}{|c|}{ Valeur } & Pourcentage \\
\hline Développement rural & 15,6 & 7,65 \\
Forêt & 1,3 & 0,64 \\
Péche & 111,9 & 54,85 \\
Transport & 7,6 & 3,72 \\
Construction & 6,0 & 2,94 \\
Energie & 7,4 & 3,63 \\
Tourisme $\quad$ & 28,0 & 13,72 \\
Santé & 21,6 & 10,59 \\
Projets régionaux & 4,6 & 2,25 \\
\hline \multicolumn{1}{|c|}{ Total } & 204 & 100 \\
\hline
\end{tabular}

Source: S.E.P.C.I., Primeiro Plano Quadrienal de Desenvolvimento Economico e Social 1983-1986, Volume 1. Relatorio Geral, Bissau, page 199. 
L'analyse de l'attribution régionale des fonds consacrés à la réalisation du Plan nous montre un net déséquilibre entre la pêche et le tourisme qui monopolisent plus de $68 \%$ du budget, et les autres secteurs qui ne disposent que de fonds marginaux. Il n'est d'ailleurs pas étonnant d'apprendre que les autorités régionales n'ont eu qu'une participation somme toute relative à l'élaboration de ce plan. ${ }^{1}$ Ainsi, leur seule participation s'est limitée, par l'entremise des Cabinets Régionaux de Planification, à préparer une liste des projets jugés prioritaires pour le développement de leur région. La liste des projets régionaux du Cabinet Régional de Planification de Bolama-Bijagos, tout comme celle de l'ensemble des autres régions, ne résiste guère à une analyse critique en terme d'impacts réels sur le développement de la région. En effet, la liste des sept projets jugés prioritaires pour la période du Plan, ${ }^{2}$ atteste que ceux-ci se concentrent quasi-exclusivement à la ville de Bolama, pour ignorer entièrement le reste de l'archipel:

1) création d'une cellule de transport à Bolama;

2) fourniture de récipients pour le transport de marchandises;

3) fourniture de bicyclettes pour les fonctionnaires;

4) creation d'un centre d'apprentissage artisanal;

5) transport pour appuyer le programme de santé de base;

6) appui à la commercialisation du poisson à Bolama; et,

7) transport collectif Bolama-São-João et île de Bolama.

La lecture du Premier Plan Quadriennal de la Guinée-Bissau nous démontre que le pays a porté l'emphase sur la mise sur pied des conditions nécessaires à assurer un minimum de cohésion dans l'appareil économique du pays. Ainsi, distribution et approvisionnement en

1 D.P.R., Proposition de projet d'appui à la DPR-MICEPCI (Divisão de Planeamento Regional): République de Guinée-Bissau, Bissau, Novembre 1985, page 13.

2 S.E.P.C.I., ep.cit., page 198. 
biens de consommation et de production, de même que libéralisation commerciale et politique financière plus rationnelle, seront apparus comme nécessaires lors de cette première phase. Le bilan macro-économique de l'Etat est malgré tout des plus modestes. La balance des paiements est déficitaire, le déficit annuel global est passé de 58 \$ millions américains en 1981 à 67 \$ millions en 1986, pour une dette externe de 320 \$ millions américains le 31 décembre 1987, alors qu'elle était de 250 \$ millions en 1983. ${ }^{1}$ La valeur des importations après la période du Plan est de cinq fois supérieure à celle des exportations, ${ }^{2}$ alors que la Plan prévoyait, pour sa variante supérieure, que les importations ne dépasseraient pas de 2,5 fois les exportations en $1986 .{ }^{3} \mathrm{La}$ consommation des particuliers devait augmenter de $27,6 \%$ entre 1984 et 1986 pour la variante supérieure ${ }^{4}$ et de $12,97 \%$ pour la variante inférieure. ${ }^{5}$ Elle s'est cependant maintenue durant cette période et est demeurée stable à 7400 P.G. per capita annuellement. ${ }^{6}$ Le niveau de réalisation des investissements pendant la période couverte par le Plan a été d'environ 8 800,1 millions de P.G. en P.G. constants de 1983, soit environ $27 \%$ de moins que ce que l'on avait prévu pour la variante inférieure. ${ }^{7}$ Il faut cependant à ce chapitre, différencier les investissements consentis par l'Etat de ceux effectivement réalisés. Ainsi, bien que les sommes accordées le furent pour 29,5\% plutôt que

1 Ministère de la Coordination Economique, du Plan et de la Coopération Internationale, La production populaire en Guinée-Bissan, Bissau, en collaboration avec OXFAM-Belgique, septembre 1985, page 10.

2 Ministério du Plano, Quarta reunião do Conselhe Consultivo do Ministério do Plano: primeira fase do II Plane Quadrienal, on.cit. page 6.

3 S.E.P.C.I., op_cit., page 78.

4 Ibid., page 72. Calculé à partir des dépenses familiales en 1984 et 1986, divisées par la population pour ces deux mêmes années (1984: 852000 personnes et 1986: 888000 personnes (Secretaria de Estado da Presidência para os Assuntos Economicos e Cooperação Internacional, Guine-Bissau: sintese da situacão económico-financeira e perspectivas de médio prazo, Bissau, fevereiro de 1987, Anexo II), ce qui nous donne une consommation annuelle per capita de 7 376,7 P.G. en 1984 et de 9 414,4 P.G. en 1986.

5 S.E.P.C.I., op. cit., page 79. En employant la même méthode que précédemment, on constate que la consommation annuelle per capita aurait dû passer de 7 376,7 P.G. en 1984 à 8 333,3 P.G. en 1986.

6 Ministério do Plano, op.cit., page 6.

7 Ibid, page 9. 
27,2\% au Bloc Prioritaire, 29,4\% plutôt que 22,5\% au Bloc d'Appui Principal, ${ }^{1}$ 18,2\% au lieu de 17,2\% pour les Secteurs Sociaux et 22,9\% aux Autres Secteurs plutôt que 33,1\%, les investissements effectivement réalisés se rapprochèrent plus des proportions des prévisions originales avec 27,03\% dans le Bloc Prioritaire, 28,84\% dans le Bloc d'Appui Principal, $17,47 \%$ dans les Secteurs Sociaux et $26,67 \%$ pour les Autres Secteurs, ainsi que le montre le Tableau 5.4.

Au niveau des sommes monétaires, les prévisions initiales du Plan accordaient la priorité au secteur de la construction et des travaux publics, suivi de celui du développement rural et, enfin, des transports. Les investissements effectivement réalisés changent peu l'ordre des choses, si ce n'est pour le secteur de la construction et des travaux publics qui a été amputé de plus de $76 \%$ de son budget initial et qui se retrouve ainsi troisième derrière le développement rural et les transports.

Le secteur de la construction et des travaux publics n'est certes pas celui qui a été le plus touché par la baisse dans les sommes investies car les secteurs de la forêt et de l'Information et Culture le précèdent. A l'inverse, les secteurs les moins frappés par la baisse des investissement s'avèrent être l'énergie, le commerce, l'administration, la Jeunesses et les Sports, et la sante; le tourisme et l'hydraulique (approvisionnement en eau), qui ont vu leurs sommes consacrées augmenter de façon substantielle et ce, tout particulièrement pour le tourisme. Le recours à l'aide externe pour le financement des projets nécessaires à la réalisation du Plan, qui devait compter pour plus de $95 \%$ des besoins monétaires, ${ }^{2}$ aura

\footnotetext{
1 I Ibidem.

2 S.E.P.C.I., op. cit., page 77.
} 


\section{TABLEAU 5.4}

Taux de réalisation des investissements par secteurs 1

Pour le Premier Plan Quadriennal

1983-1986

(en millions de P.G.)

(prix constants de 1983)

\begin{tabular}{|c|c|c|c|c|c|c|c|c|c|}
\hline \multirow[b]{2}{*}{ Secteur } & \multicolumn{2}{|c|}{ Prévisions } & \multicolumn{3}{|c|}{ Sommes disponibles } & \multicolumn{4}{|c|}{ Investissements réalisés } \\
\hline & Montent & $\%$ & Montant & $\%$ & $\begin{array}{l}\% \text { par rap- } \\
\text { port aux } \\
\text { previ- } \\
\text { sions }\end{array}$ & Montant & $\%$ & $\begin{array}{l}\text { \% por rap- } \\
\text { port aux } \\
\text { sommes } \\
\text { disponi- } \\
\text { bles }\end{array}$ & $\begin{array}{l}\text { \% par rap- } \\
\text { port aux } \\
\text { prévi- } \\
\text { sions }\end{array}$ \\
\hline BLOC PRIORTAARE & 4467,9 & 27,20 & 3306,3 & 29,46 & 74,00 & 2378,4 & 27,03 & 71,94 & 53,23 \\
\hline $\begin{array}{l}\text { Developpement Rural } \\
\text { Fore } \\
\text { Peche } \\
\text { Mines }\end{array}$ & $\begin{array}{r}2920,4 \\
555,6 \\
735,1 \\
256,8\end{array}$ & $\begin{array}{r}17,78 \\
3,38 \\
4,48 \\
1,56\end{array}$ & $\begin{array}{r}2511,2 \\
167,4 \\
470,7 \\
157\end{array}$ & $\begin{array}{r}22,38 \\
1,49 \\
4,19 \\
1,40\end{array}$ & $\begin{array}{l}85,99 \\
30,13 \\
64,03 \\
61,14\end{array}$ & $\begin{array}{r}1754,5 \\
75 \\
416,3 \\
132,6\end{array}$ & $\begin{array}{r}19,94 \\
0,85 \\
4,73 \\
1,51\end{array}$ & $\begin{array}{l}69,87 \\
44,80 \\
88,44 \\
84,46\end{array}$ & $\begin{array}{l}60,08 \\
13,50 \\
56,63 \\
51,64\end{array}$ \\
\hline BLOC D'APPUI PRINCIPAL & 3701,3 & 22,54 & 3297,9 & 29,39 & 89,10 & 2538,3 & 28,84 & 76,97 & 68,58 \\
\hline $\begin{array}{l}\text { Hydraulique } \\
\text { Trensports } \\
\text { TElecommunications } \\
\text { Commence } \\
\text { Administration }\end{array}$ & $\begin{array}{r}598 \\
2277,4 \\
260 \\
79,2 \\
486,7\end{array}$ & $\begin{array}{r}3,64 \\
13,87 \\
1,58 \\
0,48 \\
2,96\end{array}$ & $\begin{array}{r}701,2 \\
1803,6 \\
77,5 \\
58,6 \\
657\end{array}$ & $\begin{array}{r}6,25 \\
16,07 \\
0,69 \\
0,52 \\
5,85\end{array}$ & $\begin{array}{r}117,26 \\
79,20 \\
29,81 \\
73,99 \\
134,99\end{array}$ & $\begin{array}{r}782,9 \\
1225,8 \\
127,1 \\
56,8 \\
345,7\end{array}$ & $\begin{array}{r}8,90 \\
13,93 \\
1,44 \\
0,65 \\
3,93\end{array}$ & $\begin{array}{r}111,65 \\
67,96 \\
164,00 \\
96,93 \\
52,62\end{array}$ & $\begin{array}{r}130,92 \\
53,82 \\
48,88 \\
71,72 \\
71,03\end{array}$ \\
\hline SECTEURS SOCIAUX & 2828,8 & 17,22 & 2041,5 & 18,19 & 72,17 & 1537,4 & 17,47 & 75,31 & 54,35 \\
\hline $\begin{array}{l}\text { Education } \\
\text { Sante et Affaires Sociales } \\
\text { Jeunesse et Sports } \\
\text { Combattents de la Parrie } \\
\text { Information et Culure }\end{array}$ & $\begin{array}{r}698,5 \\
991,6 \\
432,4 \\
54,4 \\
100\end{array}$ & $\begin{array}{l}4,25 \\
6,04 \\
2,63 \\
0,33 \\
0,61\end{array}$ & $\begin{array}{r}606,2 \\
756,6 \\
334,8 \\
75 \\
36,8\end{array}$ & $\begin{array}{l}5,40 \\
6,74 \\
2,98 \\
0,67 \\
0,33\end{array}$ & $\begin{array}{r}86,79 \\
76,30 \\
77,43 \\
137,87 \\
36,80\end{array}$ & $\begin{array}{r}402,4 \\
642,3 \\
286,1 \\
23,5 \\
17,9\end{array}$ & $\begin{array}{l}4,57 \\
7,30 \\
3,25 \\
0,27 \\
0,20\end{array}$ & $\begin{array}{l}66,38 \\
84,89 \\
85,45 \\
31,33 \\
48,64\end{array}$ & $\begin{array}{l}57,61 \\
64,77 \\
66,17 \\
43,20 \\
17,90\end{array}$ \\
\hline $\begin{array}{l}\text { Habitat, Urbanisme } \\
\text { et Senté }\end{array}$ & 551,9 & 3,36 & 223,1 & 1,99 & 40,42 & 165,2 & 1,88 & 74,05 & 29,93 \\
\hline AUTRES SECTEURS & 5426,4 & 33,04 & 2577,2 & 22,96 & 47,49 & 2346,9 & 26,67 & 91,06 & 43,25 \\
\hline $\begin{array}{l}\text { Construction et Traveux } \\
\text { Publics } \\
\text { Energie } \\
\text { Industrie } \\
\text { Tourisme }\end{array}$ & $\begin{array}{r}3463,5 \\
506,1 \\
1014,2 \\
152,6\end{array}$ & $\begin{array}{r}21,09 \\
3,08 \\
6,17 \\
0,93\end{array}$ & $\begin{array}{l}917,3 \\
374,5 \\
693,7 \\
591,7\end{array}$ & $\begin{array}{l}8,17 \\
3,34 \\
6,18 \\
5,27\end{array}$ & $\begin{array}{r}26,48 \\
74,00 \\
68,40 \\
387,75\end{array}$ & $\begin{array}{r}820,8 \\
457 \\
494,2 \\
574,9\end{array}$ & $\begin{array}{l}9,33 \\
5,19 \\
5,62 \\
6,53\end{array}$ & $\begin{array}{r}89,48 \\
122,03 \\
71,24 \\
97,16\end{array}$ & $\begin{array}{r}23,70 \\
90,30 \\
48,73 \\
376,74\end{array}$ \\
\hline Total & 16424,4 & 100,00 & 11222,9 & 100,00 & 68,33 & 8800,1 & 100,00 & 78,41 & 53,58 \\
\hline
\end{tabular}

Source: Ministério du Plano, Quarta reunião do Conselho Consultivo do Ministério do Plano: primeira fase do II Plano Ouadrienal, Republica da Guiné-Bissau, fevereiro de 1988, 16 pages, Tableau I;

Compilation interne pour les pourcentages.

1 On constate que pour le grand axe "Autres Secteurs", il manque 290 millions de P.G. réservés aux régions; ces renseignements ne figurent pas sur le tableau original. 
vraisemblablement été responsable des quelques modifications notées entre prévisions et réalisations. L'exécution des projets prévus dans le cadre du Premier Plan Quadriennal n'est, en outre, pas seulement dépendante de l'extérieur au niveau financier, mais aussi dans le domaine des ressources humaines où la formation de la main-d'oeuvre nationale et, faut-il en convenir, la réalisation des projets, passent souvent par l'utilisation massive de l'assistance technique des organismes d'aide bi ou multi-latérale étrangers et des O.N.G.1.

En outre, comme le notent les autorités bissau-guinéennes, le choix de projets retenus pour appuyer les politiques de chacun des secteurs d'activité dans le cadre du Premier Plan, reposait dans l'existence à l'époque de l'élaboration du Plan, de ces mêmes projets ou encore, dans leur mise en branle prochaine. ${ }^{2}$ On comprendra, qu'une telle inversion dans la méthodologie d'élaboration du Plan, en plus de dénoter la constante contrainte que les problèmes d'ordre financier imposent aux autorités ainsi dépendantes des bailleurs de fonds étrangers, ${ }^{3}$ exprime la présence de malaises évidents en matière de ressources humaines dans les différents ministères et organismes impliqués. En ce sens, le manque de rigueur méthodologique, l'absence de politique concrète, ou encore les moult difficultés rencontrées dans l'élaboration et l'exécution du Plan par les différents intervenants concernés, dépendront pour une large part bien plus de problèmes liés à la formation du personnel qu'à un manque de volonté politique. Vue sous cet angle, la planification bissau-guinéenne ne se démarque guère des principales critiques formulées à l'endroit de la planification dans les pays du tiersmonde.

\section{Ibid., page 84.}

2 Ministério da Coordenação Económica, Plano e Cooperação Internacional, Linhas directrizes e grandes orientacões do II Plano de Desenvolvimento Económico e Social, Bissau, 20 de setembro de 1985, page 2.

3 Isabel Sarmento, Análise do sub-sistema de planeamento e da administracão regional da Republica da GuinéBissau, s.1., 1985, page 80 . 
Ainsi, aux réserves déjà exprimées en ce qui a trait au manque de cadres et de personnel en général, adéquatement formés, il faut ajouter les graves lacunes que connaissent ces pays en matière de données statistiques de base sur leur vie économique et sociale. ${ }^{1}$ Le Ministère du Plan de la Guinée-Bissau reconnaît d'ailleurs ce problème car il souligne la fragilité de la base statistique du pays et la faiblesse des appareils de planification et, d'une manière générale, des appareils économiques. ${ }^{2}$ La critique de Pierre Jacquemot face aux plans des pays du tiers-monde, peut s'appliquer à la Guinée-Bissau, à la lumière de ce que nous avons vu:

"Les plans observés ne sont souvent que des catalogues de projets, mal évalués au niveau des coûts directs et des charges récurrentes, non hiérarchisés l'un par rapport à l'autre et présentés en ordre dispersé dans des politiques sectorielles qui ne révèlent aucun véritable choix stratégique. Les financements des projets inscrits sont en outre la plupart du temps aléatoires. Le cadrage macroéconomique et macrofinancier est absent ou, au mieux, à peine esquissé et ne résulte pas d'une recherche systématique des cohérences entre projets et moyens disponibles. Dans le même ordre d'idées, il est souvent reproché au planificateur de preférer les grands projets publics et les objectifs ambitieux sans souci de réalisme et sans s'inquiéter de l'emploi optimal des ressources." 3

Dans un ordre d'idées théorique, nous pouvons classifier les plans selon qu'ils soient impératifs, c'est-à-dire qu'ils imposent un système de régulation précis, centralisé, et qu'ils s'effectuent à priori de la production, ou encore qu'ils soient indicatifs parce qu'ils ne forment en fait qu'une série de mesures incitatives et non-autoritaires. ${ }^{4}$ Le Ministère du Plan de la Guinée-Bissau affirme et souligne pour sa part, le caractère impératif de sa planification. ${ }^{5}$ La pratique ne sera certes pas convaincante en la matière. N'avons-nous à ce

\footnotetext{
1 Pierre Jacquemot, "Crise et renouveau de la planification du développement", Revue Tiers-Monde, Tome XXV, No. 98, avril-juin 1984, page 253.

2 Ministério da Coordenação Económica, Plano e Cooperação Internacional, op. cit., page 3.

3 Pierre Jacquemot, op. cit., page 253.

4 P. Hugon, O. Sudrie, "La crise de la planification africaine, diagnostic et remedes", Revue Tiers-Monde, Tome XXVIII, No. 110, avril-juin 1987, page 411.

5 S.E.P.C.I., op cit. page 7.
} 
titre qu'à faire référence aux méthodes d'élaboration et d'exécution du Premier Plan, et à sa grande dépendance face à l'étranger, ou encore à l'ignorance, de la part de la très grande majorité des fonctionnaires rencontrés, des différentes facettes du Plan concernant leur ministère et les modalités d'exécution qui s'y rattachent. En ce sens, la planification bissauguinéenne correspond assez fidèlement aux principales critiques que l'on adresse à la planification de l'ensemble du continent:

"Conçus de manière technocratique, les plans africains ne jouent ni le rôle incitatif et concertatif de la planification indicative ni le rôle impératif et structurant de la planification centralisée. Ils constituent le plus souvent, soit des catalogues de projets extérieurs sans véritable coordination (cas des pays à libéralisme planifié) soit des objectifs cohérents sans véritables projets (cas des économies se réclamant du marxisme-léninisme).

Dans la pratique, peu de plans africains respectent les trois principes d'optimisation, de cohérence et de régulation:

- le principe d'optimation n'est pas retenu; les modèles utilisés sont prévisionnels ou décisionnels et non d'optimation. De nombreux plans ne posent pas la question de l'allocation intemporelle des ressources (choix d'actualisation, gestion des ressources non-renouvelables) ou privilégient des choix interrégionaux pour des raisons sociopolitiques sans en évaluer les coûts économiques et financiers; les objectifs sont définis sans hiérarchie ni priorités;

- le principe de cohérence est souvent absent, soit parce que les projets disponibles ne cadrent pas avec des cohérences macroéconomiques, soit parce que le pays ne dispose pas de documents permettant le cadrage macroéconomique (absence de TES, de comptabilité nationale, de modèles macroéconomiques et financiers);

- le principe de régulation est rarement présent: les instances de planification sont moins des lieux de pilotage pour maintenir le cap que des traducteurs de changements de cap, compte tenu des instabilités sociopolitiques internes et des fluctuations extérieures." 1

Considéré sous cet angle, le plan bissau-guinéen pourrait être placé à mi-chemin entre l'indicatif et l'impératif, celui-ci conservant son caractère a priori et centralisé, mais, faute de moyens, ne pouvant faire force de loi, si ce n'est dans les livres officiels. Il n'en constitue pas moins un excellent indicateur des politiques que favorisent l'Etat et le Parti et, en ce sens, devrait être reconnu à sa juste valeur comme garant (du moins en principe) des actions que

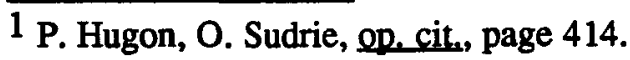


tout intervenant en matière de développement pourrait entreprendre à sa suite. Les renseignements qu'offre le Plan sur les avenues possible de développement du pays et de ses régions, peuvent s'avérer des alliés indiscutables si on ne le remise pas aux oubliettes et si on ne fait pas trop de cas de son manque de rigueur. Il importe donc de le considérer comme un indicateur de la politique officielle du pays et des objectifs de développement que celui-ci entend poursuivre ou, du moins, vers lesquels il tend.

Il peut être intéressant de retourner au bilan du Premier Plan Quadriennal pour remarquer que l'objectif d'atteinte de l'autosuffisance alimentaire du pays peut sembler être en bonne voie de réalisation. Effectivement, les estimations du Ministère du Développement Rural indiquent l'atteinte des 120000 tonnes de riz comme production pour $1986,{ }^{1}$ objectif que se fixait le Plan 2 qui espérait ainsi voir les besoins en matière d'aide alimentaire être inférieurs en 1986 à ceux de $1984 .{ }^{3}$ Sur ce point aussi, le Plan peut avoir atteint ses objectifs, la balance alimentaire nationale en riz et en autres céréales n'accusant qu'un déficit de 28022 tonnes en 1986 alors qu'en 1984, il s'elevait à 53470 tonnes. $^{4}$

Pour ce qui est des sept projets prévus pour la région de Bolama, seulement deux auront été mis en branle: la commercialisation du poisson est entre les mains de Pescarte, et un traversier effectue la liaison Bolama-São-João. Les autre projets n'ont cependant pas été réalisés et nous aurons l'occasion de revenir sur le sujet lors du sixième chapitre. Quoiqu'il en soit, l'attribution à chaque région d'une enveloppe régionale destinée à l'exécution de son propre plan régional de développement, représente en soi un effort notable de régionalisation

1 Secretaria de Estado da Presidência para os Assuntos Economicos e Cooperação Internacional, op.cit., Anexo II.

2 S.E.P.C.I., op.cit., page 99.

3 Ibid, page 77.

${ }^{4}$ Secretaria de Estado da Presidência para os Assuntos Economicos e Cooperação Internacional, Guine-Bissau: sintese da situação económico-financeira e perspectivas de médio prazo, op.cit, Anexo II. 
du système de planification national d'après Isabel Sarmento qui s'est livré à une analyse de ce système de planification. 1

Le Premier Plan concentra donc ses objectifs vers les domaines de la production, de la distribution et des finances, pour ignorer presqu'entièrement les problèmes sociaux du pays, que ce soit au niveau de la santé, de l'éducation, de l'exode rural ou encore du chômage dans les villes. Cependant, le Deuxième Plan Quadriennal doit avoir une philosophie différente du Premier Plan qui recherchait l'autosuffisance alimentaire, la satisfaction des besoins du marché intérieur et une plus grande stabilité économique. Le Deuxième Plan devrait correspondre, dit-on, à une phase de construction nationale et de développement autonome où l'on pourra porter davantage l'emphase sur les secteurs sociaux. ${ }^{2}$

5.1.2 Le Deuxième Plan Quadriennal de Développement Economique et Social

\subsubsection{Prévisions}

Dans les prévisions initiales du gouvernement, un second plan devait suivre le premier pour les années 1987 à 1990.3 En février 1988, le Second Plan Quadriennal n'était toujours pas prêt et, de ce fait, il est prévu qu'il s'étalera des années 1989 à 1992. ${ }^{4}$ Ainsi, des trois

1 Isabel Sarmento, op. cit., pages 69-70.

2 Savane Landing, BNA-Guinée-Bissau, rapport final, s.1., 1983, pages 26-29.

3 Ibid., page 29.

4 Secrétariat d'Etat à la Présidence pour les Affaires Economiques et la Coopération Internationale, Etude préliminaire sur l'amélioration de l'organisation et du fonctionnement de SOLIDAMI. Document de travail. s.l., $1^{\circ}$ juin 1988 , version No. 2 , page 5. 
grandes étapes nécessaires à l'élaboration du deuxième plan, le gouvernement n'en était-il qu'à la seconde, la première étant terminée et la dernière restant toujours à faire: ${ }^{1}$

- élaboration d'un rapport général d'encadrement;

- préparation d'un rapport relatif à la planification en tant que telle; et,

- élaboration du document final du Second Plan Quadriennal devant être soumis à l'approbation et aux critiques de l'Assemblée Nationale Populaire.

A l'époque de la réalisation des recherches relatives au mémoire (1986 à 1988), bien que le Second Plan n'était pas encore prêt, les principales orientations étaient connues puisqu'un document sur ses grandes lignes directrices paraissait à la fin de 1985.2

Soulignant une fois de plus la nécessité du Second Plan, ${ }^{3}$ le document relève ce qu'il considère comme constituer les deux principales caractéristiques du pays, sa forte dépendance face à l'extérieur que ce soit dans les domaines économiques, financiers ou techniques, et la fragilité de ses appareils statistiques et économiques. ${ }^{4}$ De là découle l'objectif principal de ce plan, qui sera non seulement de consolider les mesures de stabilisation déjà entreprises et de rechercher l'équilibre dans la balance des opérations courantes, mais surtout de viser l'augmentation de la production du pays grâce à une politique axée principalement sur le développement des zones rurales. ${ }^{5}$

Le Premier Plan nous avait déjà mis sur la piste d'un effort nécessaire de développement du monde rural; le Deuxième Plan ne fait que confirmer l'importance du

\footnotetext{
1 Ministério du Plano, Quarta reunião de Conselho Consultive de Ministério de Plano: primeira fase do II Plano Ouadrienal, op cit., page 2.

2 Ministério da Coordenação Económica, Plano e Cooperação Internacional, op. cit., 41 pages.

3 Ibid., pages 3-4.

4 Ibid., page 3.

5 Ibid., page 6.
} 
monde rural. En fait, la Guinée-Bissau espère pouvoir éviter les échecs des politiques de planification entreprises dans les divers pays africains du sud du Sahara ces 25 dernières années, qui ne se sont soldées que par: ${ }^{1}$

- une baisse dramatique et continue de la production agricole de subsistance qui s'explique soit par la primauté accordée par ces Etats aux cultures d'exportation, seules capables de fournir en devises ces pays, soit par des politiques de prix tenus artificiellement bas afin de faciliter l'approvisionnement des milieux urbains; et,

- une urbanisation disproportionnée due à un exode rural massif de population ayant perdu leurs systèmes de production traditionnels au profit des secteurs secondaire et tertiaire très peu productifs.

Le Ministère du Plan souligne que la Guinée-Bissau conserve encore des atouts majeurs qui pourraient lui permettre d'échapper à ce sort puisqu'on note la survie en campagne des modes de production traditionnels et l'urbanisation du pays somme toute assez limitée. ${ }^{2} \mathrm{~A}$ partir de ce raisonnement, le rôle majeur que l'on compte attribuer au monde rural s'explique, et l'orientation que l'on se fixe pour ce Deuxième Plan se résume à la "consolidação da estabilização e obtenção do equilíbrio das operações correntes, no quadro de uma política principalmente centrada no desenvolvimento das zonas rurais". 3

Faire du développement rural le centre de la politique de développement poursuivi dans le cadre du Bloc Prioritaire, le Deuxième Plan étant composé des mêmes axes que le

\footnotetext{
1 Ibid, page 7.

2 Ibidem.

3 Ibid, pages 7-8: "consolidation de la stabilisation et obtention de l'équilibre des opérations courantes, dans le cadre d'une politique principalement centrée dans le développement des zones rurales" (traduction de l'auteur).
} 
Premier, ${ }^{1}$ trouve son fondement dans le fait que malgré les discours officiels, depuis le début du Premier Plan Quadriennal, les dépenses de développement de ce secteur n'ont fait que diminuer au profit des zones urbaines. ${ }^{2}$ Bien que nous ne disposons d'aucune donnée précise sur l'affectation réelle des investissements de développement effectués en régions, il n'en demeure pas moins qu'une analyse des prévisions du Premier Plan démontre que les quatre zones du pays, qui comptent pour $86 \%$ de la population, ne jouissent que de $36 \%$ des investissements prévus alors que le Secteur Autonome de Bissau, avec les $14 \%$ de la population totale du pays, recueille $24 \%$ des investissements. Autre fait intéressant à souligner, les sommes prévues pour ce que l'on appelle "Ambito Nacional" (intégralement "Sphère Nationale") et que l'on peut associer à l'administration du pays, cumulent $40 \%$ des investissements prévus. ${ }^{3}$ On sait pertinemment que ces sommes auront des retombées directes sur les différents centres administratifs urbains et vraisemblablement bien peu à la campagne.

Le Deuxième Plan innove par rapport au Premier, en incitant le désengagement de l'Etat de certains secteurs d'activité où son intervention n'est pas prioritaire. Il compte, de cette façon, céder la gestion d'unités de ces secteurs au secteur privé, national ou étranger et ce, de préférence, sous forme de sociétés mixtes. ${ }^{4}$ Ce désengagement, animé par le désir de rentabiliser ces activités, du moins de façon minimale, ${ }^{5}$ prend sa source dans l'autocritique de l'Etat que sait effectuer une de ses branches, le Minsitère du Plan:

\footnotetext{
1 Ibid., page 18.

2 Ibid., pages 18-19.

3 S.E.P.C.I., op cit., page 203.

${ }^{4}$ Ministério da Coordenação Económica, Plano e Cooperação Internacional, op. cit, page 9.

5 Ibidem.
} 
"A semelhança de muitos outros países do nosso Continente, o aparelho de Estado é uma das causas principais das nossas dificuldades actuais: a sua hipertrofia, a sua inadaptação as necessidades do desenvolvimento, a tendência para escolher as soluçōes expontaneamente, indo os projectos ao encontro dos seus interesses. Se estas causas não caracterizam menos - a níveis diferentes diversos sectores do aparelho de Estado.

Se queremos evitar que esta situação hipotéque seriamente o nosso desenvolvimento, devemo-nos comprometer a fazer uma profunda reforma, com vista a transformar a Administração Pública num instrumento ao serviço da colectividade, do seu bem estar e do seu progresso." 1

Outre l'adoption de nouvelles règles de conduite administratives face aux salariés de l'Etat et la recherche de l'augmentation de leur productivité, cette transformation de l'appareil d'Etat doit passer, aux dires mêmes du Ministère du Plan, par un renforcement de la prise en considération des réalités régionales et locales. ${ }^{2}$

La régionalisation du développement, qui est intégrée à ce stade dans l'élaboration du Deuxième Plan, passe par le renforcement et l'émancipation des organes du pouvoir local. ${ }^{3} \mathrm{~A}$ ce chapitre, nous pouvons comprendre la création des provinces qui, grâce à leurs cabinets de ministre résident remplaceront efficacement espère-t-on les comités d'Etat régionaux, trop préoccupés, comme nous le verrons, à préserver et défendre leurs intérêts locaux, sans aucune véritable vision régionale du développement. L'intégration des régions au processus national d'élaboration du Deuxième Plan Quadriennal s'est faite dans les années 1987-1988, sous la forme de réunions spéciales dans les cabinets des ministres résidents qui portaient sur

\footnotetext{
1 Ministério da Coordenação Económica, Plano e Cooperação Internacional, op.cit, pages 10-11:

"A l'image de nombreux autres pays de notre continent, l'appareil d'Etat est l'une des principales causes de nos difficultés actuelles: son hypertrophie, son inadaptation aux besoins du développement, sa tendance à choisir les solutions spontanément, entraînent l'apparition de projets à l'encontre de ses intérêts. Si ces causes ne sont pas générales, elle n'en caractérisent pas moins - à différents niveaux - les divers secteurs de l'appareil d'Etat.

Si nous voulons éviter que cette situation n'hypothèque sérieusement notre développement, nous devons nous engager dans une profonde réforme ayant pour but de transformer l'Administration Publique en un instrument au service de la collectivité, de son bien-être et de son progrès" (traduction de l'auteur).

2 Ibidem.

3 Ibidem.
} 
les différents projets régionaux à inclure dans le Plan. Le sixième chapitre du mémoire reviendra d'ailleurs sur ces événements pour la Province du Sud.

Certes, l'un des points les plus originaux de ce plan, concerne l'emphase que l'on espère accorder aux formes collectives ou associatives, notamment au niveau de la production agricole, des échanges (commerce et transport) et, plus tard, des activités de transformation. ${ }^{1}$ Les autorités escomptent cependant éviter un développement incontrôlé du secteur privé qui pourrait provoquer, de leurs dires mêmes, une augmentation excessive des inégalités qui se traduiraient principalement par une déstructuration de l'économie rurale. ${ }^{2}$ C'est pourquoi l'Etat veut assumer un certain contrôle du passage du privé au public et en plus garder le monopole de certains secteurs stratégiques essentiels comme les activités bancaires, les postes et télécommunications, l'énergie, le transport aérien, le commerce externe, les ressources naturelles et surtout le contrôle de la gestion de l'économie nationale. ${ }^{3}$

Au niveau macro-économique, le Deuxième Plan prévoit la continuation des efforts d'assainissement entrepris lors du Premier Plan: limitation des dépenses étatiques, augmentation des recettes de l'Etat, assainissement du secteur public et limitation du financement étatique des projets au profit de l'aide externe. ${ }^{4}$

Le Deuxième Plan Quadriennal, se distingue donc du plan précédent par l'attention particulière qu'il entend porter aux nécessités élémentaires de la population (santé, éducation, énergie, approvisionnement en eau et habitation), ${ }^{5}$ et par le développement des associations

\footnotetext{
1 Ibid, page 10.

2 Ibidem.

3 Ibid, page 9.

4 Ibid., pages 14 et 17 .

5 Ibid., page 16.
} 
afin d'engager la population dans l'économie monétaire, ${ }^{1}$ pour ainsi mieux intégrer la campagne et les villes dans des liens d'échange réciproques garantissant le plus possible l'absence de marges de profits excessives que pourrait entraîner par exemple un système commercial typiquement prive. ${ }^{2}$ De même $1^{\prime E}$ tat aspire-t-il à partager certains coûts nécessaires à la réalisation de ses objectifs avec la population où, par exemple dans les domaines des services de base, les associations de village pourraient coopérer en fournissant main-d'oeuvre, appui logistique au personnel de l'Etat, etc. ${ }^{3}$.

\subsubsection{La production populaire}

L'augmentation de la production en Guinée-Bissau se heurte à plusieurs contraintes dont la technologie peu développée et dépendante et la difficulté d'approvisionnement des producteurs en matière première et en biens de production. Cette carence en matière d'approvisionnement découle tant du monopole qu'exerçait jusqu'à récemment l'Etat sur les devises étrangères que des carences propres au système de commercialisation et de distribution. Cette pénurie, qui affecte l'ensemble du pays, pose un problème de taille à quiconque, individu, association ou projet, se propose d'oeuvrer dans les domaines de la production, qu'elle soit artisanale ou industrielle.

Dans les grandes lignes directrices du Deuxième Plan Quadriennal, il est souvent fait mention, au même titre que les associations de producteurs, d'un secteur de l'économie bissau-guinéenne, que l'on appelle production populaire. Terme typiquement bissau-guinéen, on pourrait associer la production populaire à ce que l'on appelle dans d'autres pays,

\footnotetext{
1 Ibid., page 19.

2 Ibid, page 28.

3 Ibid., page 31.
} 
"économie populaire" ou "économie informelle" qui, outre les activités reliées directement à la production en elle-même, s'inscrit dans celles plus larges de la commercialisation des produits ainsi obtenus. La production populaire en Guinée-Bissau comprend aussi les activités situées en aval et en amont de la production puisqu'elles sont souvent prises en charge par le producteur même. ${ }^{1}$

Le développement de ce secteur dépendait de l'accueil que lui ferait le Parti. ${ }^{2}$ Lors du Quatrième Congrès du P.A.I.G.C. de novembre 1986, on reconnut au secteur de la production populaire un rôle majeur dans le développement du pays, comme le précisent les Résolutions générales du Congrès:

..."O IV ${ }^{\circ}$ Congresso do PAIGC:

1. decide que o desenvolvimento económico e social do país se faça no quadro de uma economia mixta, onde participarão todos os agentes económicos.

2. estabelece em matéria de desenvolvimento económico as seguintes directivas gerais a serem levadas à prática pelos orgãos executivos do Estado:

a. a criação de condições para um aumento sustentado e contínuo da produção e das colheitas agrícolas;

b. atracção dessa produção para os circuitos oficiais de comercialização, tanto os voltados para o abastecimento e consumo interno, como os ligados a exportação; c. incentivar outras actividades locais tais como a pescatoria, a produçã̃o popular e artesanal;

d. garantir um abastecimento normal e regular do sector produtivo moderno em matéria prima, em energia, em sobressalentes e outros;

e. fomentar a pesquisa aplicada como factor inovador e dinamizador de desenvolvimento económico e social;

f. desenvolver a produção, com particular atenção a produção popular." 3

1 Ministère de la Coordination Economique, du Plan et de la Cooperation Internationale, La production populaire en Guinée-Bissau, op cit page 3.

2 Ibid., pages 6 et 9.

3 Grupo Dinamizador, "Algumas conclusð̃es do PAIGC sobre a produção popular no IV ${ }^{\circ}$ Congresso de Novembro de 1986", Produção Popular. Boletim de Informação, No. 1, março de 1987, page 15:

..."Le IV ${ }^{\circ}$ Congrès du P.A.I.G.C.:

1. décide que le développement économique et social du pays se fait dans le cadre d'une économie mixte, où participent tous les agents économiques.

2. établit, en matière de développement économique, que les directives générales suivantes doivent être mises en pratique par les organes exécutifs de l'Etat:

a. la création des conditions pour une augmentation substantielle et continue de la production et de la cueillette agricole; 
La production populaire devient ainsi la pierre angulaire de la politique bissauguinéenne de développement de la production. Elle se caractérise par certaines particularités. ${ }^{1}$ Tout d'abord, elle exige peu de capital et généralement une importante main-d'oeuvre. Elle s'exerce ensuite habituellement à partir de techniques et d'instruments traditionnels dont le rendement est régulièrement faible.

Enfin, puisque les différentes productions populaires sont plus ou moins d'ordre traditionnel, elles relèvent souvent de la culture même des ethnies qui la pratiquent. Leurs modalités (méthodes de production, méthodes d'écoulement ou de commercialisation de la production, etc.) sont relativement rigides et ne se transmettent pas facilement d'un groupe social, d'une ethnie ou encore d'un sexe à l'autre.

La production populaire regroupe les classes d'activité suivantes: ${ }^{2}$

- la transformation des produits agricoles;

- les diverses formes d'artisanat;

- la construction;

- la mâtrise de l'eau et de l'énergie;

- la pêche artisanale; et,

- les transports.

b. la destination de cette production vers les circuits officiels de commercialisation tant orientés vers la fourniture et la consommation interne que l'exportation;

c. stimuler les autres activités locales comme la pêche, la production populaire et artisanale;

d. garantir un approvisionnement normal et régulier du secteur productif moderne en matière première, en énergie, en pièces de rechange et autres;

e. favoriser la recherche appliquée comme facteur d'innovation et de dynamisation du développement économique et social;

f. développer la production en portant une attention particulière à la production populaire" (traduction de l'auteur).

1 Ibidem.

2 Ibidem. 
A la lumière de ces quelques explications sur la production populaire, nous pouvons la définir en Guinée-Bissau, de la manière suivante:

"A produção popular é a transformação de produtos agrícolas, pesqueros e animais, a fabricaçã̃o de meios de produção - principalmente para a agricultura e a pesca - e de outros bens de consumo de primeira necessidade, destinados às famílias, associações de tabanca e mercados locais, graças à exploração dos recursos naturais e humanos do país, na base de tecnologias tradicionais, eventualmente melhoradas, cujo benefício se destina individualmente ou colectivamente aos próprios produtores. E desencadeada pelas próprias forças produtivas ao nível da tabanca ou do bairro urbano e implica a forma de propriedade e organização de produção tradicional, uma dependência reduzida do material importado e um domínio das tecnologias utilizadas pelos produtores." 1

Bien que la production populaire n'apparaisse officiellement en Guinée-Bissau que lors de l'élaboration du Deuxième Plan Quadriennal, on peut dire que depuis le début des années 1980, une certaine dynamique s'est peu à peu développée en sa faveur. Plusieurs projets se sont depuis lors intéressés à la production populaire et ce, à trois niveaux principaux, soit l'approvisionnement en matières premières et en outillage, l'amélioration des technologies existantes et, enfin, l'introduction de nouvelles technologies. ${ }^{2}$

De l'avis de responsables de plusieurs de ces projets, il appert qu'ils connaissent souvent des problèmes immenses au niveau de l'écoulement de leur production, faute de réseaux de transport ou de circuits de commercialisation. En outre, les projets concernés font parfois face à une concurrence majeure de la part de produits semblables, importés ou fournis

\footnotetext{
1 Grupo Dinamizador, op. cit., page 3:

"La production populaire est la transformation de produits agricoles, de la pêche ou animaux, la fabrication de moyens de production - principalement pour l'agriculture et la pêche - et d'autres biens de consommation de première nécessité, destinés aux familles, associations de village et marchés locaux, grâce à l'exploitation des ressources matérielles et humaines du pays, sur la base de technologies traditionnelles, éventuellement améliorées, dont le bénéfice est destiné imdividuellement ou collectivement aux producteurs mêmes. Elle est déclenchée à partir des forces productives du village ou du quartier urbain et implique les formes de propriété et d'organisation de la production traditionnelle, une dépendance réduite en matériel importé et des technologies familières aux producteurs" (traduction de l'auteur).

2 Ibid., page 20.
} 
au pays à titre de dons, mais dont le prix de vente est nettement inférieur au prix de revient des produits fabriqués sur place.

Il n'en demeure pas moins que l'ouverture de l'Etat bissau-guinéen à la production populaire et, plus largement au droit de libre association pour fins de production, de distribution ou de commercialisation, marque le début d'une ère qu'il importe d'exploiter à sa juste valeur. A ce titre, la production populaire constitue à notre avis une alternative intéressante à l'augmentation de la production du pays et à l'intégration de la population dans le circuit économique national par rapport à la politique de développement par pôles de croissance industriels que poursuivait le gouvernement de Luis Cabral.

\subsection{Les structures régionales de planification}

Le Ministère du Plan de la Guinée-Bissau est responsable de l'élaboration et de l'exécution du plan national de développement. Son rôle déborde cependant cette fonction de préparation, de coordination et de contrôle de son exécution, pour s'appliquer aussi à la supervision dans la préparation et l'exécution de programmes multi-sectoriels, à la définition du plan d'information statistique et de l'exécution des opérations relatives au système statistique national et, enfin, à l'approbation du programme statistique national. ${ }^{1}$ Pour ce faire, le Ministère du Plan utilise deux des structures de son organigramme, la Direction Générale de Planification (Direcção Geral de Planeamento, D.G.P.) et la Direction Générale de la Statistique (Direcção Geral da Estatistica, D.G.E.). ${ }^{2}$

\footnotetext{
1 Isabel Sarmento, Análise do sub-sistema de planeamento e da administração regional da Republica da GuinéBissau, op. cit., page 17.

2 Ibid., pages 18 et 21 .
} 
Seule la Direction Générale de la Planification possède des ramifications régionales grâce à la Division de Planification Régionale, de Programmation et Projets, et de Planification Sectorielle (Divisão de Planificação Regional de Programação e Projectos e de Planificação Sectorial) qui, dans le cadre de la Division de Planification Régionale (Divisão de Planificação Regional, D.P.R.) et de la Division de Planification Sectorielle (Divisão de Planeamento Sectorial, D.P.S.), oeuvre dans les différents secteurs et régions du pays. 1 Notre objectif étant avant tout d'ordre régional, nous allons nous attarder plus particulièrement sur le cas des D.P.R..

\subsubsection{La Division de Planification Régionale (D.P.R.)}

Les structures régionales de planification furent formées en 1979 à partir des anciennes structures régionales du Commissariat d'Etat à la Coordination Economique et au Plan, qui avait, à l'époque, essentiellement pour fonction la cueillette des impôts et le travail de recensement ${ }^{2}$ dont celui de la population dans le cadre du Recensement Général d'avril 1979 et celui du bétail de $1976 .{ }^{3}$ Les D.P.R., qui s'appelaient à l'époque D.D.R. (Departamento de Desenvolvimento Regional, Département de Développement Régional), héritèrent ainsi en régions, de tout le personnel de l'ancien C.E.C.E.P. ${ }^{4}$.

La création des D.P.R. marque un changement majeur dans la politique de développement jusque là monopolisée par la présence de grands projets centralisés à Bissau. 5

\footnotetext{
1 Ibid., pages 18-20.

2 Ibic., page 40.

3 Renaldo de Araujo Lima, Elementos para uma programacão deso trabalhos do sistema de planificacão regional. 1981/1982, Bissau, Ministério da Coordenação Económica e do Plano, Departamento de Desenvolvimento Regional, 1981, page 4.

4 Ibidem.

5 Isabel Sarmento, op. cit., page 40.
} 
Elle vise à "permettre aux instances politico-administratives régionales (aux niveaux des tabancas, secteurs et régions) de mieux exprimer leurs besoins, leurs nécessités, et leurs souhaits spécifiques de développement". 1

La structure régionale du Ministère du Plan est ainsi composée d'une Délégation de Planification Régionale (D.P.R.) sous la responsabilité d'un délégué régional par région, et d'autant de Délégations de Planification Sectorielle et de délégués sectoriels que la région compte de secteurs. Dans le cas de la région Bolama-Bijagos, on note la présence d'un délégué régional à Bolama et de quatre délégués sectoriels (Bolama, Bubaque, Uno, Caravela).

Les fonctions officielles des D.P.R. consistent à: ${ }^{2}$

- mâ̂triser la connaissance de la réalité régionale au moyen de collecte de données, d'études et analyses socio-économiques;

- identifier les potentialités de développement de leur région et de leurs secteurs administratifs;

- rechercher les points d'étranglement du développement de leur région et de ses secteurs;

- proposer aux instances supérieures des moyens d'appuyer les potentialités ou encore d'éliminer les points d'étranglement du développement de leur région et de ses secteurs;

- proposer aux instances supérieures des moyens de doter les populations de conditions techniques et financières nécessaires à la promotion de leur développement;

1 Ibidem.

2 Renaldo de Araujo Lima, op.cit., pages 4-5. 
- proposer aux agents du gouvernement des moyens et des instruments favorisant l'intégration des politiques de développement national au développement régional;

- présenter au gouvernement des propositions de plans, de programmes et de projets de développement régional;

- fournir au Ministère du Plan les informations de base et les études socioéconomiques indispensables à la coordination économique, au développement et à la préparation des Plans nationaux;

- aider les différents ministères à s'intégrer à la politique et aux activités régionales de développement;

- assurer l'élaboration, le contrôle de l'exécution et l'évaluation des différents projets de développement régional; et,

- prêter assistance à l'administration régionale en matière de planification régionale.

A ces fonctions importantes des D.P.R., s'ajoutent celles propres au travail des délégués régionaux qui, en plus d'avoir à coordonner et à veiller sur les activités des délégués sectoriels de leur région, doivent assumer le rôle de secrétaire du cabinet régional de planification. ${ }^{1}$ De plus, comme tout membre d'organe régional, ils doivent pouvoir répondre de leurs activités aux comités d'Etat régionaux. ${ }^{2}$

Afin d'assurer les conditions nécessaires à la mise en place de ces structures de planification régionale, le Ministère du Plan a pu bénéficier de l'appui d'un projet du gouvernement hollandais qui, de 1979 à 1983, a permis l'installation et la fourniture en

1 Isabel Sarmento, op.cit., pages 26-27.

2 D.D.R., Desenvolvimento regional: Programa Bienal de Desenvolvimento 1980/1981, Bissau, Commissariado de Estado da Coordenação Económica e Plano, maio de 1980, page 4. 
équipement de huit départements dans les régions, l'acquisition de moyens de transports dans les régions, et la création d'un centre de documentation à Bissau qui recueille et diffuse diverses informations d'ordre économique. De plus, le projet s'est soldé par l'envoi d'un certain nombre d'assistants techniques au sein des régions afin, entre autres, de remédier aux carences notables du personnel en matière de formation. ${ }^{1}$

Une fois l'assistance hollandaise parvenue à son terme, les D.P.R. en régions se sont retrouvées dans une situation de paralysie presque complète malgré l'équipement dont elles pouvaient disposer vu l'absence de financement national pour leurs besoins de fonctionnement notamment en combustible ou en pièces de rechange pour les véhicules. ${ }^{2}$

Il n'y a pas qu'au niveau physique que la paralysie affecte les D.P.R.. En effet, l'influence des assistants techniques en région a été positive, du moins pour le temps que dura leur intervention. On souligne qu'un seul des quatre délégués régionaux qui a joui de l'appui d'un assistant technique a pu être en mesure de poursuivre ses fonctions de façon autonome mais a, depuis lors, quitté son poste pour oeuvrer au sein d'un projet de développement. $^{3}$

La période suivant l'aide hollandaise aura donc été marquée d'une forte chute des capacités de la part de la D.P.R. en général, d'effectuer ses tâches. Ainsi, et pour affecter encore plus le travail des régions, on constate le manque d'orientation et d'information entre la D.P.R. centrale à Bissau et les D.P.R. régionales, le tout combiné à une absence totale d'encadrement des tâches. ${ }^{4}$ Tout ceci fait dire à Isabel Sarmento que, depuis la fin de l'aide hollandaise:

\footnotetext{
1 Isabel Sarmento, op. cit., pages 43-46.

2 Ibid, page 45.

3 Ibid, page 57.

4 Ibid., page 58.
} 
"E pois uma situação de letargia que subsiste a partir dessa data no DDR (actual DPR) situação minimizada pela iniciativa dos seus elementos activos, mas cujo esforço, acaba por ser limitado por toda a falta de apoio, enquadramento e definição de funções." 1

Le bas niveau de formation des membres régionaux des D.P.R. ne fait qu'ajouter aux difficultés de fonctionnement de ces structures et l'on perçoit même un sentiment d'exclusion de ses cadres de la part de leurs collègues des autres ministères, qui n'y trouvent pas là d'interlocuteurs valables à leurs yeux. ${ }^{2}$

La région de Bolama ne fait pas exception à la situation de léthargie qui affecte l'ensemble des D.P.R. du pays: absence d'encadrement de la part de Bissau, personnel très peu formé, manque de dynamisme et de motivation, absence de moyens de transport ou de budget de transport, tous ces maux forment le lot du sort quotidien de la D.P.R. BolamaBijagos. On ajoute à cela des communications difficiles entre Bolama et les trois autres secteurs (à Uno et Caravela, il n'y pas de réseau téléphonique), et on a une bonne idée de la situation précaire qui prévaut à Bolama. ${ }^{3}$ Vu sous cet angle, et sans bonification majeure des Délégations de Planification Régionale et de leur coordination à Bissau, on ne peut que penser que la régionalisation du système de planification bissau-guinéen ne pourra être véritablement à la hauteur de la tâche technique qu'exige la planification, et ce, avant longtemps.

1 Ibid., page 45:

"Il s'agit donc d'une situation de léthargie qui subsiste depuis cette date à la D.D.R.(actuel D.P.R.), situation minimisée par l'initiative de ses éléments actifs, mais dont l'effort a fini par être limité par le manque d'appui, d'encadrement et de définition de fonctions"(traduction de l'auteur).

2 Ibid, pages 112-113.

3 Alain Rousseau, Rapport final d'activité. Projet d'appui à la Délégation Régionale du Ministère du Plan de Bolama-Bijagos: juillet 1986 à juillet 1988. Bolama, C.E.C.I., juin 1988, 13 pages + Annexes. 


\subsubsection{Les cabinets régionaux de planification (G.R.P.)}

Les cabinets régionaux de planification ("Gabinete Regional de Planificação", G.R.P.) furent créés à partir de 1980 et constituent les organes régionaux de planification de premier plan. Fonctionnant en théorie sur la base de rencontres régulières, ces cabinets réunissent les autorités régionales (président et secrétaire du comité d'Etat régional), le délégué régional du Plan, les délégués régionaux des différents ministères et, éventuellement, les représentants d'autres organismes ou projets régionaux, tous sous la présidence du président du comité d'Etat de la région. ${ }^{1}$

Initialement créés pour appuyer la préparation du Premier Plan Quadriennal en produisant un diagnostic de leur région, ${ }^{2}$ les G.R.P. doivent maintenant en principe délibérer sur les différents sujets relatifs à la stratégie de développement de la région, dans son ensemble et pour chacun des organes locaux de l'Etat, afin de déboucher sur un Plan Régional dont ils devront veiller à l'exécution, tout comme ils devront favoriser l'émergence de différents petits projets de développement. ${ }^{3}$

Dans la pratique, une fois les travaux du Premier Plan complétés, les G.R.P. se sont retrouvés sans appui technique de la part de Bissau. Il en découla d'importantes différences entre les régions au niveau du fonctionnement et de l'efficacité de ces cabinets qui ne reposèrent plus, à partir de ce moment, que dans le dynamisme de leur président ou dans celui du représentant du Ministère du Plan. Dès lors, les cabinets des différentes régions espacèrent de plus en plus leurs réunions et ne purent plus tenir les réunions mensuelles qu'on avait initialement prévues. 4

1 Isabel Sarmento, op. cit. page 24.

2 Ibid., page 59.

3 Ibid., pages 24-25.

4 Ibid., pages 59-60. 
Ainsi, à titre d'exemple, le G.R.P. de Bolama, créé en septembre 1982, ne s'est-il réuni que cinq fois de 1982 à 1987 (septembre 1982, octobre 1982, septembre 1985, octobre 1986 et mars 1987) et aucune de ces rencontres n'a mobilisé l'ensemble des délégués ministériels de la région. 1

Isabel Sarmento souligne que les cabinets régionaux de planification sont aussi affectés par le manque de formation de leurs membres et l'absence de liens entre ceux-ci et les représentants des projets en région. Ainsi, les Présidents se retrouvent-ils dans l'impossibilité de traiter des sujets relatifs au développement de leur région, les délégués de D.P.R. qui devraient compiler les renseignements nécessaires, identifier les goulots d'étranglement et la problématique régionale de développement, se trouvant dans l'incapacité de leur fournir l'appui nécessaire. S'ajoute à cela la grande diversité des membres des G.R.P. tant en matière de formation qu'en ce qui a trait à leurs champs d'intérêt, ce qui ne fait qu'aviver des conflits. Ainsi, par exemple, les délégués de la santé et du développement rural, qui sont généralement beaucoup plus formés que les autres membres du cabinet, acceptent peu le bien-fondé de ces réunions qu'ils ne considèrent que comme une tribune au service du Ministère du Plan. ${ }^{2}$

Il en découle que les G.R.P. s'éloignent de plus en plus de leur rôle de lieu de rencontre, d'échange et d'orientation sur les problèmes de développement local, pour ne s'occuper qu'à traiter de problèmes ponctuels, de l'ordre de ceux qui affectent personnellement les membres, et qu'à aborder les projets régionaux selon les seules informations dont ils peuvent disposer. Ces informations sont d'ailleurs généralement fort limitées vu la faible participation des délégués régionaux ou encore l'absence de lien d'information entre Bissau où se prennent les décisions et la région. De plus, lorsqu'il s'agit

1 D.P.R.-Bolama-Bijagos, Pasta \# 9: Actas, Bolama.

2 Isabel Sarmento, op. cit. pages 62-64. 
d'identifier des projets d'envergure régionale, les G.R.P. ne considèrent que ceux d'ordre local, et assurent leur financement, soit grâce aux enveloppes régionales dont ils disposent, soit, lorsque le projet est de dimension plus imposante, par l'entremise du ou des ministères concernés qui peuvent ainsi l'exécuter. Enfin, dans le cas des projets liés à des activités de production en tant que telles et impliquant plus spécifiquement les populations locales, on remarque que leur élaboration ne se fait pas au sein des G.R.P., mais plutôt dans diverses O.N.G., ambassades ou encore dans le cadre du travail d'assistants-techniques du D.P.R.; dans ces cas, le G.R.P. ne tient lieu que de réunion d'information. ${ }^{1}$

La formulation des priorités régionales par les G.R.P. relève donc des intérêts propres de ses membres et nous aurons l'occasion de nous en apercevoir plus en détail avec l'exemple pratique que constituera l'analyse du G.R.P. Bolama-Bijagos. Tout ceci ne fera que confirmer les allégations selon lesquelles, sans un meilleur encadrement, les G.R.P. sont condamnés à demeurer les tribunes d'une poignée de fonctionnaires. Ainsi note-t-on à propos de ces cabinets:

"Il apparaît une certaine contradiction entre les objectifs formulés par exemple, dans les plans de développement nationaux et favorisés par les donateurs (spécialement les ONG), et les besoins spécifiques des autorités régionales.

Les premières donnent la priorité aux projets directement productifs ou à une meilleure participation de la population au niveau des tabancas, les dernières pensent plutôt à un aménagement de l'infrastructure, spécialement dans la capitale régionale: construction de maisons pour les fonctionnaires ou d'un club pour la JAAC, un terrain de football, etc.." 2

L'échec actuel de cette structure de planification régionale est évidente. Seul un organe véritablement représentatif des structures locales pourrait s'avérer plus efficace. Pour ce faire,

${ }^{1}$ Ibid., pages 62-63.

2 D.P.R., Proposition de projet d'appui à la DPR-MICEPCI (Divisão de Planeamento Regional): République de Guinée-Bissau, op. cit., pages 12-13. 
il faudrait donc procéder à la création de G.R.P. élargis où s'ajouteraient aux autorités locales et aux représentants des délégations, des représentants d'organisation de masses, ce qui se fait déjà à l'occasion, de même que des membres de projets ou d'entreprises importantes, sans omettre de laisser une place aux comités ou associations de tabancas pour y faire connaître leurs besoins. Isabel Sarmento souligne cette nécessité et attribuerait à ces nouveaux cabinets les fonctions suivantes: ${ }^{1}$

- proposer et formuler les résolutions de plan régional et les soumettre au conseil régional;

- formuler les orientations de base pour la stratégie à observer dans le cadre du développement de la région;

- assurer la cohérence des activités des différents ministères en région; et,

- assurer le suivi de l'exécution du plan régional et des projets existants.

Il n'en demeure pas moins que toute tentative d'intégration des cabinets régionaux de planification dans le volet régional de la planification du pays, en plus de s'attaquer aux lacunes strictement techniques, doit tenir compte de la réalité politique du pays. En effet, l'association qui prévaut à tous les niveaux administratifs entre le Parti et l'Etat, se retrouve aussi dans ces structures régionales, le président du cabinet étant président du comité d'Etat régional et membre du Parti. Cette constatation, loin de constituer une critique, implique néanmoins qu'au sein des G.R.P., les présidents jouissent d'un statut particulier obligeant respect des membres face à celui que l'on nomme le "gouverneur". L'expérience aura démontré que cette reconnaissance de ce rôle suprême du président en région pose une entrave certaine au libre jeu de la discussion et des négociations entre les membres. Ce

\footnotetext{
1 Ibid., page 122.
} 
monopole de pensée et d'action dans ces structures de développement régional biaise effectivement le jeu des forces en présence.

\subsection{SOLIDAMI, création d'une O.N.G. bissau-guinéenne}

Les activités de coopération internationale sont courantes en Guinée-Bissau. Elles regroupent autant les formes d'aide bi et multilatérales, que les actions menées par les nombreuses O.N.G. disséminées sur son territoire. Alors que les premières se spécialisent dans les projets de grande envergure impliquant le recours à d'importants budgets, les secondes se spécialisent dans des...

..."projets de petite et de moyenne dimension, à investissement réduit et avec une gestion légère, où la participation populaire est une composante indispensable, raison pour laquelle ce genre de coopération répond d'une forme adéquate et directe aux besoins fondamentaux des populations." 1

Si l'on prend en compte le fait que le gouvernement a clairement signifié ses politiques de recours massif à l'aide externe pour l'appuyer dans ses efforts de développement, on comprend aisément les désirs de coordination et de supervision qu'il entend effectuer auprès de cette cinquantaine d'O.N.G. oeuvrant dans le pays. ${ }^{2}$ Plusieurs ne fournissent d'ailleurs qu'un appui trop ponctuel ne permettant que le démarrage du projet et non sa consolidation et sa poursuite, comme ce fut le cas du Projet de Développement Agricole Bolama-Bijagos, alors que d'autres vivent dans un isolement des plus complets 3 où aucune coordination n'existe avec d'éventuels projets voisins ou encore avec leur ministère de tutelle. C'est la

1 Guinée-Bissau, Conférence des Organisations Non Gouvernementales: document d'orientation, op. cit.,, page 51.

2 Tbid., page 36.

3 Ibid., page 45. 
raison pour laquelle on a créé, en avril 1984, le SOLIDAMI (Solidariedade e Amizade) chargé de la "coordination des aides extérieures non gouvernementales".1

SOLIDAMI qui, aux dires mêmes de ses créateurs, doit constituer "l'embryon d'une future ONG guinéenne", 2 est pour le moment lié au Secrétariat d'Etat à la Présidence pour les Affaires Economiques et la Coopération Internationale (S.E.P.A.E.C.I.). Ce secrétariat d'Etat est apparu en 1986 suite au retrait du Ministère de la Coordination Economique, du Plan et de la Coopération Internationale (MICEPCI) des tâches de coordination des aides externes qui lui étaient jusque là dévolues, pour se voir confirmer dans son rôle de planificateur du développement économique national sous le nom de Ministère du Plan. ${ }^{3}$

Depuis 1986, SOLIDAMI est composé de deux cellules, l'une chargée des études et des projets regroupant les attributions relatives à la coordination de l'aide non-gouvernementale, et l'autre chargée de la gestion administrative et financière de l'institution. ${ }^{4}$ SOLIDAMI n'a pas pour but de remplacer les différents départements d'Etat dans le rôle de coopération avec les différentes O.N.G. qu'ils effectuent déjà (cas des ministères de tutelle avec lesquels chaque projet doit collaborer) mais compte plutôt jouer un rôle de soutien et de promotion. 5 En fait, ses principales fonctions sont: 6

- d'assurer l'étude et le suivi des projets;

- de fournir l'appui à la mise en oeuvre de projets;

1 Secrétariat d'Etat à la Présidence pour les Affaires Economiques et la Coopération Internationale, Etude préliminaire sur l'amélioration de l'organisation et du fonctionnement de SQLIDAMI. Document de travail.op.cit., page 3.

2 Guinée-Bissau, op.cit., page 52.

3 Secrétariat d'Etat à la Présidence pour les Affaires Economiques et la Coopération Internationale, op.cit., page 5.

4 Ibid, page 6.

5 Guinée-Bissau, op. cit., page 55.

6 Secrétariat d'Etat à la Présidence pour les Affaires Economiques et la Coopération Internationale, op. cit., pages 8-10. 
- d'effectuer la prospection, la promotion, la mobilisation et le suivi des O.N.G.; et,

- d'effectuer la diffusion de la documentation et de son traitement informatique.

SOLIDAMI semble bien engagé dans le processus d'étude et de suivi des différentes interventions des O.N.G. sur le territoire national comme en fait foi l'importante somme de données qu'il a recueillies sur le terrain. Dans les cas de mise en oeuvre de nouveaux projets ou encore de recherche de sources de financement, il est difficile, faute de données ou d'exemples précis, d'en évaluer la véritable portée. Cependant, au niveau de la coordination des activités des O.N.G., le cas de la région Bolama-Bijagos entre autres, confirme qu'aucun effort notoire n'a pu être mené à terme. Il faut dire à ce sujet que la coordination des O.N.G. et de leurs actions ne peut être envisagée sans "l'adhésion consciente et enthousiaste des ONG". ${ }^{1}$ On peut craindre que nombre d'O.N.G. n'émettent des réserves face à tout effort de coordination externe à leurs services et l'exemple de Bolama aura démontré que les O.N.G., qui n'ont bien souvent de véritables liens de communication qu'entre leur siège social et leur projet sur le terrain, s'isolent peu à peu des autórités locales et nationales. Ce repli sur soi s'explique soit par un sentiment d'incompréhension de leurs activités par les structures administratives nationales, soit, tout simplement, par méfiance envers ces structures et esprit de compétition avec les projets des autres O.N.G.. Ainsi, les responsables d'lle de Paix interprètent-ils les activités de SOLIDAMI:

"Il semblerait que la volonté politique du Secrétariat d'Etat à la Coopération Internationale soit d'en faire plutôt un organe de contrôle qu'un organe de coordination, à travers une ONG nationale. Malgré l'existence du SOLIDAMI, le danger de voir certains programmes se superposer ou s'organiser à partir de logiques contradictoires reste présent." 2

1 Guinée-Bissau, op.cit., pages 65-66.

2 Philippe de Braconier, Elizabeth Drory, Rapport de mission de juin 1985: préétude de l'lle de Paix de Bolama, op. cit., page 5. 
On ne peut douter qu'une telle perception du rôle de SOLIDAMI, si elle est courante chez les O.N.G., ne fasse que marginaliser son travail et poser de graves contraintes aux quelconques efforts de coordination qu'il pourrait entreprendre, s'il ne sait imposer impérativement son autorité. 


\section{CHAPITRE SIX}

\section{POPULATION LOCALE ET DEVELOPPEMENT PLANIFIE}

Les deux derniers chapitres avaient pour but d'une part de nous familiariser avec les structures administratives du pays et leur fonctionnement et, d'autre part, d'exposer les orientations nationales en matière de politique de développement socio-économique. Pour ce faire, nous avons dû nous éloigner de notre terrain d'étude régional, l'île de Bolama, pour focaliser notre attention sur l'ensemble du pays. Il est maintenant temps de revenir au terrain concerné afin qu'à la lumière des considérations évoquées dans cette deuxième partie du mémoire, nous puissions évaluer la portée des pratiques de l'Etat et des différents projets de coopération en regard des populations locales, de leurs besoins et de leurs aspirations. Ainsi seulement, une fois que nous aurons pu identifier ou, du moins, pris connaissance des divergences (si divergences il y a) entre les perceptions des différents partis, pourrons-nous évaluer les modalités d'un développement valable. On ne souviendra certes que nous avions associé développement à prise en charge par une population de la satisfaction de ses intérêts et de ses besoins. Cette capacité d'une société de se prendre en main, qui ne peut que passer par une implication générale dans le processus et l'identification des besoins de la collectivité, ne pourra, comme nous avons pu nous en apercevoir, se refléter dans la seule croissance quantitative des revenus et de la consommation, et la généralisation de l'économie monétaire 
qui ignoreraient les particularités ethno-culturelles de la population ou, pis encore, les nivelleraient dans un moule commun.

Amilcar Cabral, définissait la culture comme

..."la synthèse dynamique, au niveau de la conscience de lindividu ou de la collectivité, de la réalité historique, matérielle ou spirituelle, d'une société ou d'un groupe humain, des relations prévalant aussi bien entre l'homme et la nature qu'entre les hommes et entre les catégories sociales. Les manifestations culturelles sont les différentes formes par lesquelles cette synthèse s'exprime, individuellement ou collectivement, à chaque étape de l'évolution de la société ou du groupe humain en question." 1

II soulignait qu'en Afrique, le temps de la colonisation n'avait pas été suffisant pour détruire de façon significative les "éléments essentiels de la culture et des traditions" 2 des peuples colonisés. De là constatait-il l'importance qu'avait pu jouer, dans l'affirmation de la lutte pour son identité qu'a mené le peuple bissau-guinéen contre la domination étrangère, le support principal de la culture, la structure sociale. ${ }^{3}$ Il nous apparaît dès lors évident que le rôle actif que la culture a eu au sein de la lutte de libération nationale prouve, à notre avis, hors de tout doute qu'elle doive continuer de s'inscrire dans cette deuxième phase de la lutte qu'est la reconstruction nationale, recherche d'un développement harmonieux.

Une prise en compte des réalités culturelles ethniques doit donc primer, tout comme la reconnaissance du caractère dual de Bolama où deux mondes coexistent sans vraiment entrer en contact. Tout modèle de développement devra, à ce niveau, refléter les intérêts tant du monde urbain que du monde rural, sans privilégier l'un au détriment de l'autre. De même, ce modèle doit se baser sur le respect des habitudes et des conduites propres aux sociétés verticales comme horizontales. Une société comme la société balante sera-t-elle rébarbative au concept de chef? Très bien, il n'en tient alors qu'au modèle de développement de s'adapter et

\footnotetext{
1 Amilcar Cabral, Unité et lutte, Paris, Petite Collection Maspero, 1980, page 186.

2 Ibid., page 175.

3 Ibid., page 181.
} 
de trouver comment composer avec cette réticence à l'autorité. De la même manière devra-t-il pouvoir canaliser l'habitude d'organisation et de hiérarchisation des sociétés verticales. Le véritable défi, en ce sens, sera évidemment de concilier ces réalités, tout en respectant les structures et les formes de pensée traditionnelles propres à chaque ethnie et en harmonie avec le milieu.

\subsection{Les aspirations locales}

Lors de notre enquête de tabanca, nous nous enquérions auprès des membres des comités de tabanca, de leurs vues en matière de développement de leurs communautés villageoises. Les résultats obtenus nous parurent concluants et dénotent, à nos yeux, une connaissance réaliste de la part des comités de tabanca des besoins réels des villages. En effet, en aucun cas nous n'avons eu affaire à des demandes portant sur des actions ayant très peu d'incidence sur l'ensemble du village pour ne concerner qu'une minorité.

Comme le démontre le Tableau 6.1, les demandes les plus fréquentes touchent l'aménagement de puits et le développement de la pêche. A l'époque où furent menées les enquêtes, l'installation de puits fermés dans les villages ne faisait que commencer. Depuis lors cependant, l'ensemble de lîle a pu bénéficier de ce programme. Il reste par contre à vérifier, à plus long terme, quelle sera la durabilité des mécanismes de pompage manuel utilisés et quelles seront les modalités relatives à la fourniture des pièces de rechange éventuellement nécessaires et au recours aux réparateurs. 
TABLEAU 6.1

Tableau de fréquence des principales demandes de développement selon le nombre de villages

Enquête de tabanca Ile de Bolama 1987

\begin{tabular}{|l|r|}
\hline \multicolumn{1}{|c|}{ Nature de la demande } & Nombre de villages \\
\hline Aménagement du puit & 15 \\
\hline Développement de la pêche & 9 \\
\hline Concasseur de noix de palme & 5 \\
\hline Réparation de la rizière & 5 \\
\hline Pharmacie ou poste de sante & 5 \\
\hline Presse à huile & 3 \\
\hline Matériel agricole et semences & 3 \\
\hline Ecole & 3 \\
\hline Traction animale & 2 \\
\hline Amélioration de la route & 2 \\
\hline Radio V.H.S. & 2 \\
\hline Transport & 2 \\
\hline Lutte contre les prédateurs & 1 \\
\hline
\end{tabular}

Source: D.P.R.-Bolama-Bijagos, Inquérito de tabanca, Bolama, avril 1987. 
En deuxième lieu vinrent les demandes concernant le développement de la pêche. Dans ces cas précis, la fourniture de matériel (filets, lignes, hamę̧ons) importait tout autant, aux yeux des responsables des comités de tabanca, que l'encadrement, par un projet de pêche, des activités de pêches elles-mêmes. Au moment de l'enquête, le projet de Pescarte-Bolama n'avait pas encore commencé à fonctionner pour appuyer les pêcheurs professionnels. Quant à l'initiative de fourniture de matériel et d'encadrement de la pêche artisanale dans les villages par Ile de Paix, elle non plus n'avait pas encore débuté. La demande et le besoin de petit matériel de pêche sont cependant importants à Bolama, comme l'atteste le cas de ce village mandingue, Uato Mandinga, où huit pêcheurs se retrouvaient en chômage technique, faute de matériel.

$\mathrm{Au}$ troisième rang des demandes villageoises figurait le besoin de concasseur de noix de palme. Cette demande provenait des villages situés à l'extrémité sud de l'île, une zone où les palmiers sont relativement abondants puisqu'on y retrouve deux anciennes plantations (Gâ Moria et Uato Fula) dont l'exploitation plus intensive nécessiterait certes un travail d'amélioration. Cinq villages en ont exprimé le besoin. Il est intéressant de noter qu'utilisées conjointement avẹc les presses à huile, dont le besoin a été identifié dans trois villages de la même zone, les concasseurs de noix peuvent permettre de maximiser l'extraction d'huile. ${ }^{1}$ Bien qu'à Bolama, l'exploitation du palmier ne jouisse d'un potentiel moins élevé que dans d'autres parties du pays dont l'archipel bijago en tant que tel, il n'en demeure pas moins qu'une certaine bonification, tant des méthodes que des moyens de travail peut avoir sa place,

\footnotetext{
1 L'extraction de l'huile de palme peut s'effectuer en deux étapes. La première étape consiste à séparer les noix palmistes de leur enveloppe de laquelle on obtient une huile rouge, de consommation très courante dans les zones riches en palmiers. Cette huile, est fabriquée par les femmes aidées des enfants, peut être produite avec ou sans presse à huile, le pressage de la pulpe pouvant s'effectuer avec les mains. Les noix palmistes doivent être cassées si l'on veut utiliser leur amande pour obtenir une huile claire, de meilleure qualité, et que l'on appelle "huile blanche"; pour ce faire, l'utilisation d'une concasseuse est nécessaire.
} 


\section{TABLEAU 6.2}

Répartition des principales demandes

selon le village

Enquête de tabanca

Ile de Bolama

1987

\begin{tabular}{|c|c|c|c|c|c|}
\hline Nature de la demande & Villag & s concernés & Nature de la demande & Vullaq & 3 concernés \\
\hline \multirow[t]{3}{*}{ Probleme de puits } & \multirow{3}{*}{$\begin{array}{l}\text { Arenja Vida } \\
\text { Balanbar } \\
\text { Bairro Assomada } \\
\text { Bolama de Baixo } \\
\text { Calidje } \\
\text { Canema } \\
\text { Ga Nafa } \\
\text { Lala Balmenta }\end{array}$} & \multirow{3}{*}{$\begin{array}{l}\text { Madina } \\
\text { NTatcha } \\
\text { Ponta Apili } \\
\text { Ponta Bissau } \\
\text { Ponta Doce } \\
\text { Pontia } \\
\text { Parmgal } \\
\text { Uato Fula } \\
\end{array}$} & $\begin{array}{l}\text { Pharmacie ou poste } \\
\text { de santc }\end{array}$ & $\begin{array}{l}\text { G\& Moria } \\
\text { Madina } \\
\text { Marsasse } \\
\end{array}$ & $\begin{array}{l}\text { Ponta Bissau } \\
\text { Ponta Doce }\end{array}$ \\
\hline & & & Presse a huile & $\begin{array}{l}\text { Bolama de Baixo } \\
\text { Calidje }\end{array}$ & Ponta Doce \\
\hline & & & & & \\
\hline \multirow[t]{3}{*}{$\begin{array}{l}\text { Développement de la } \\
\text { peche }\end{array}$} & \multirow{3}{*}{$\begin{array}{l}\text { Bolama de Baixo } \\
\text { Calidje } \\
\text { Casseque } \\
\text { Ga Beafada } \\
\text { Lala }\end{array}$} & \multirow{3}{*}{$\begin{array}{l}\text { Madina } \\
\text { Ponta Apili } \\
\text { Ponta Doce } \\
\text { Pujumguto } \\
\text { Uato Mandinga }\end{array}$} & $\begin{array}{l}\text { Materiel agricole et } \\
\text { semences }\end{array}$ & $\begin{array}{l}\text { Cassucal } \\
\text { Ponta Bissau } \\
\end{array}$ & Pontia \\
\hline & & & Ecole & $\begin{array}{l}\text { Bolama de Baixo } \\
\text { Casseque }\end{array}$ & Marsasse \\
\hline & & & & & \\
\hline \multirow{2}{*}{$\begin{array}{l}\text { Concasseuse de noix } \\
\text { de paime }\end{array}$} & \multirow[b]{2}{*}{$\begin{array}{l}\text { Bolama de Baixo } \\
\text { Calidje } \\
\text { Madina }\end{array}$} & \multirow[b]{2}{*}{$\begin{array}{l}\text { Ponta Doce } \\
\text { Uato Fula }\end{array}$} & Traction animale & $\begin{array}{l}\text { Luanda } \\
\text { Madina } \\
\end{array}$ & Marsasse \\
\hline & & & $\begin{array}{l}\text { Amelioration de } \\
\text { la roure }\end{array}$ & Balanbar & Lala \\
\hline \multirow{3}{*}{$\begin{array}{l}\text { Reparation de la } \\
\text { rizitere }\end{array}$} & \multirow{3}{*}{$\begin{array}{l}\text { Conemn } \\
\text { Lals Balenta } \\
\text { Luonda }\end{array}$} & \multirow{3}{*}{$\begin{array}{l}\text { Madina } \\
\text { Ponta Doce }\end{array}$} & Radio VHS & Madina & Ponta Doce \\
\hline & & & Transport & G8 Moria & Pontia \\
\hline & & & $\begin{array}{l}\text { Lutte contre les } \\
\text { prédateurs }\end{array}$ & Aranja Vida & \\
\hline
\end{tabular}

Source: D.P.R.-Bolama-Bijagos, Inquérito de tabanca, Bolama, avril 1987. 
ne serait-ce que pour faciliter le travail des villageois et éventuellement assurer un certain approvisionnement à la ville de Bolama.

La réparation des rizières, qui arrive ex aequo dans les demandes villageoises avec le besoin de concasseur de noix de palme, constitue, tout comme la fourniture en matériel agricole et en semences, un besoin que l'on peut qualifier de fondamental, vu l'importance que revêt l'agriculture dans le mode de vie de la population locale. D'ailleurs faut-il le remarquer une fois de plus, le rôle majeur que la population accorde au riz dans son régime alimentaire implique que le développement de cette culture revêt à ses yeux une importance primordiale. Vu sous un angle économiste, l'autosuffisance alimentaire en riz ne perd en rien de son caractère impératif, la dépendance du pays envers l'aide alimentaire internationale étant notoire. Par exemple, le pays reçoit d'importantes quantités d'aliments soit gratuitement à titre de don, soit commercialement, à prix très "compétitif". Il revend en partie ces aliments par la suite, afin de payer les coûts de transport et de manutention d'une part, et de renflouer les caisses de l'Etat d'autre part. Le Programme Alimentaire Mondial, le P.A.M., fait parvenir à la Guinée-Bissau le nécessaire pour assurer une ration familiale mensuelle de $45 \mathrm{~kg}$ de riz, 12,5 $\mathrm{kg}$ de sorgho, $5 \mathrm{~kg}$ de poisson en boîte et de 3,5 litres d'huile. ${ }^{1}$ En pratique, le programme ne s'applique qu'aux fonctionnaires qui peuvent acheter riz et occasionnellement huile, par l'intermédiaire du comité d'Etat. Le reste semble être vendu dans les différents commerces et marchés du pays. Cependant, aucune donnée sur le sujet n'a été trouvée. On comprendra que cette aide, qui présente certains caractères la rapprochant au "dumping", rendue nécessaire par le déficit alimentaire du pays, s'avère être une arme à double tranchant qui cause une concurrence déloyale à la production locale en tuant dans l'oeuf toute tentative de relance de la production agricole. Une entente contractée entre lle de Paix et le P.A.M.

1 Hannes Stegemann, Ile de Paix de Bolama rapport de mission à Bolama. 03/04/87-23/05/87, Huy, Iles de Paix, 1987, page 17. 
assurait jusqu'en 1988, l'approvisionnement gratuit des employés du projet en ces différents produits. ${ }^{1}$ Avec la libéralisation du commerce et le meilleur approvisionnement des marchés locaux qui s'en est suivi, l'arrêt de cette distribution alimentaire gratuite par les dirigeants du projet, bien qu'ayant dans un premier temps, on s'en doute, créé un certain mécontentement chez les employés-bénéficiaires, représente une action positive dans le sens du développement de la production et de la distribution locales.

La faveur populaire en matière de développement ou d'appui à l'agriculture se traduit par le fait que cinq villages expriment clairement leurs désirs de voir effectuer les travaux nécessaires à la réfection de leurs rizières endommagées et que trois villages soulignent leur manque de matériel agricole (essentiellement du petit matériel) et de semences de riz, d'arachides et de jardin. Il peut sembler paradoxal de constater le faible nombre de villages faisant état de leur besoin de faire réparer leur rizière, si l'on tient compte que l'enquête aura permis l'identification de 12 villages possédant des rizières abandonnées (voir Tableau 6.3). Dans ces cas, les causes d'abandon relevaient pour $57,1 \%$ des raisons, à des bris de digue entraînant l'invasion de l'eau salée, pour $21,4 \%$, à la sécheresse, le reste se divisant également entre le manque de moyen (manque de ressources humaines), la guerre 2 et les insectes.

Le Projet de Développement Agricole Bolama-Bijagos aura concentré ses efforts sur ces deux problèmes de réfection des rizières et de fourniture de matériel et de semences. Cependant, la réparation de rizières n'aura pas touché tous les villages qui en avaient exprimé le besoin car, souvent, le manque de main-d'oeuvre disponible au village, conséquence de l'exode rural, ou encore le peu de dynamisme évident de certains villages, freinent toute

1 Ibid., page 30.

2 La guerre aura, dans bien des cas, forcé la population, par manque de main-d'oeuvre, à abandonner la culture des rizières, ce qui a entraîné un abandon qui de provisoire est devenu permanent. 
tentative de travail. Un village même, Ponta Doce, ayant mobilisé les efforts du projet dans la réfection d'une de ses digues, a préféré opter pour une méthode relevant de l'expertise traditionnelle vis-à-vis d'un problème technique particulier, plutôt que de se fier à celle du projet. A la suite d'une forte marée, la digue se rompit et l'eau salée anéantit ainsi les efforts de toute une saison de travail. L'année suivante, des conflits familiaux au sujet de la somme de travail de chaque famille, et un certain fatalisme face aux mauvais esprits, vinrent perturber et paralyser les tentatives de réparation de cette digue. Seuls quelques paysans purent alors récolter du riz. 1

La nécessité pour certains villages de se voir doter d'un poste de santé revêt la même importance aux yeux des villageois que la présence d'un concasseur de noix dans le village ou la réparation de la rizière. L'importance qu'accordent les villageois à l'existence d'un poste de santé et, dans une moindre mesure, à celle d'une école, n'étonne guère lorsque l'on connaît les distances que les villageois ont à parcourir pour avoir recours à ces services, tout comme l'ont attesté leurs demandes de moyens de transport et d'amélioration de la route. Le besoin de développement des transports ne fait de doute. Les deux villages qui ont formulé cette proposition se trouvent de l'autre côté de l'île par rapport à la ville de Bolama, seul endroit où ils peuvent écouler leur production maraîchère pour Pontia et celle de fruits de cocotiers et de palmiers pour Gâ Moria. Quant à l'amélioration de la route, problème qui va de pair avec le précédent, les deux villages requérants sont d'accès routier des plus difficiles. Ainsi on ne peut accéder à Balanbai que par un sentier impraticable par voiture alors que la route menant à Lala est parsemée de nombreuses et importantes trappes de sable.

1 Entrevue avec Paul Rouillard, chef du Projet de Développement Agricole Bolama-Bijagos, Bolama, 2 juin 1988. 
TABLEAU 6.3

Causes d'abandon des rizières

par village

Ile de Bolama

1987

\begin{tabular}{|l|l|}
\hline \multicolumn{1}{|c|}{ Nom du village } & \multicolumn{1}{|c|}{ Cause(s) d'abandon } \\
\hline Aranja Vida & Invasion de l'eau salée \\
Bairto Assomada & Guerre \\
Bolama de Baixo & Invasion de l'eau salée \\
Calidje & $\begin{array}{l}\text { Sécheresse } \\
\text { Insectes }\end{array}$ \\
Canema & Invasion de l'eau salée \\
Lala Balanta & Invasion de l'eau salé \\
Madina & $\begin{array}{l}\text { Invasion de l'eau salé } \\
\text { Manque de moyens }\end{array}$ \\
Marsasse & Invasion de l'eau salé \\
Ponta Doce & Invasion de l'eau salée \\
Pontia & Invasion de l'eau salée \\
Uato Fula & Sécheresse \\
Uato Mandinga & Sécheresse \\
\hline
\end{tabular}

Source: D.P.R.-Bolama-Bijagos, Inquérito de tabanca, Bolama, avril 1987. 
Le désir de voir se développer la traction animale s'explique facilement, hormis par les services évidents que ce mode de traction peut offrir au niveau des transports et de l'agriculture, par la composition ethnique de deux des villages qui ont formulé la demande, Uato Fula et Madina. En effet, les Foulas sont, comme nous l'avons noté, traditionnellement éleveurs. Lors de nos visites au village de Madina, le président du comité de tabanca nous a appris que du temps des Portugais, le village avait déjà été l'objet d'un projet de traction animale. Malheureusement pour les villageois, les deux boeufs affectés par le projet leur ont été volés, dit-on, par des Balantes des villages voisins. Il est intéressant de noter à ce titre que les Balantes jouissent d'une réputation de voleurs qui n'est d'ailleurs certes pas étrangère au fait que lors de leurs épreuves d'initiation, les jeunes Balantes se doivent de commettre des larcins pour prouver leur intelligence, leur habilité et leur courage.

La requête exprimée par deux villages de se voir doter d'une radio V.H.S., repose cependant sur des considérations qui pourraient nous apparaître moins pertinentes, et exprime clairement une certaine convoitise que ces villages entretiennent avec celui de Uato Fula où le Projet de Développement Intégré des Iles Bijagos (P.D.I.I.B.) de Bubaque a installé l'un des deux postes émetteurs-récepteurs de l'île (l'autre étant situé à Bolama) visant à relier les îles de l'archipel par un réseau de télécommunication.

Enfin, pour ce qui est de la lutte contre les prédateurs et les insectes, il est remarquable qu'elle ne préoccupe qu'un seul village étant donné le manque évident de produits phytosanitaires dans le pays et la présence de nombreux prédateurs. 


\subsection{Les propositions du G.R.P.}

Si les demandes villageoises nous ont semblé pertinentes dans l'ensemble puisqu'elles sous-tendaient un impact général au niveau du village, dans les domaines de l'hygiène et de la santé (cas des demandes de puits et de postes de santé), dans ceux de la production alimentaire (développement de la pêche, de l'agriculture, de l'exploitation du palmier, de la lutte contre les prédateurs), des transports (traction animale, amélioration de la route, développement des transports) ou encore de l'éducation, nous ne pouvons en dire autant des propositions faites par le Cabinet Régional de Planification de Bolama-Bijagos depuis sa fondation, et plus encore des projets qu'il a effectivement mis en branle.

En 1982, lors de sa première réunion, le G.R.P. de la région Bolama-Bijagos a adopté des statuts lui conférant les responsabilités suivantes: ${ }^{1}$

- formuler un plan régional de développement faisant partie du plan quadriennal national;

- étudier et proposer les orientations de base de la stratégie régionale de développement;

- assurer la cohérence et la coordination des actions régionales et locales des programmes des différents ministères;

- gérer l'exécution du plan régional; et,

- proposer et formuler des projets de développement dans la région et tout particulièrement des projets d'assistance aux villages.

Afin de rendre opérationnelles ses actions, le G.R.P. dispose d'une somme d'argent réservée à l'exécution des priorités régionales qu'il a lui-même identifiées. Ce fonds, le

1 Gabinete Regional de Planificação da Região de Bolama-Bijagos, Estatutos do Gabinete Regional de Planificação de Bolama-Bijagos, Bolama, 5 de outubro de 1982, page 2. 
F.N.I.("Fundo Nacional de Investimento", Fonds National d'Investissement) est attribué par le Ministère du Plan par l'intermédiaire du Ministère des Finances ${ }^{1}$ et est géré par le Comité d'Etat Régional, 2 qui l'administre selon les priorités qu'il a su dégager.

Nous avons déjà pris connaissance des priorités régionales identifiées dans le cadre du Premier Plan Quadriennal au Chapitre cinq, et constaté qu'hormis celles dont l'exécution relevait du niveau central à Bissau, aucune n'avait été réalisée. Il convient d'ajouter dans le cas de Bolama, que malgré l'identification de balises en matière de développement régional au sein du plan quadriennal, le G.R.P. dresse une liste de projets qu'il juge prioritaire au développement de la région et que cette liste ne s'avère aucunement en accord avec celle du Premier Plan Quadriennal. De cette liste, et en fonction des sommes reçues, le G.R.P. et le Comité d'Etat Régional veillent à la réalisation de ce qui leur semble le plus urgent. L'expérience nous aura appris, ayant assisté à quelques réunions du G.R.P. de Bolama, qu'en fait, le Président décide unilatéralement et sans aucune consultation, de l'affectation de ces sommes.

Un relevé des différents rapports relatifs à l'utilisation du F.N.I. dans la région de Bolama-Bijagos, nous renseigne sur les quelques faits suivants: 3

- 12 novembre 1984, réception de 750000 P..G pour la réparation de la piscine municipale de Bolama (vestige de l'époque coloniale);

- 22 février 1985, attribution de la moitié des fonds nécessaires à la réparation du cinéma de Bolama ("Clube" de Bolama) (3 783 124 P.G.) et du "Jardim Público Infantil "(1 908000 P.G.) (Jardin d'enfants);

\footnotetext{
1 D.P.R.-Bolama-Bijagos, Pasta \#16:FNI, Bolama, 29 de janeiro de 1985.

2 Luis Napoleão dos Reis, Relatório da Delegacia do Plano da regiăo de Bolama-Bijagos do ano de 1987. Bolama, D.P.R.-Bolama-Bijagos, 1987, page 2.

3 D.P.R.-Bolama-Bijagos, on.cit.
} 
- 25 mai 1985, déblocage de 2000000 P.G. pour le "Clube" de Bolama et le "Jardim Público Infantil";

- 16 juillet 1987, réception de 8850000 P.G. pour les travaux relatifs à la réparation de la maison des visiteurs (logement pour fonctionnaires, membres du Parti et autres invités officiels), du siège du Parti et du Secrétariat du Comité d'Etat de Bolama;

- 17 novembre 1987, sollicitation de fonds supplémentaires pour la réparation du siège du Comité d'Etat de Bubaque; et,

- sollicitation, le 10 janvier 1988, du besoin de 332636 325,80 P.G., dont 50\% en devises, pour la réalisation des travaux suivants:

1) achèvement des travaux du siège du Parti de Bolama,

2) achèvement des huit logements pour fonctionnaire à Bubaque;

3) réparation et fourniture d'équipement du Secrétariat et de la résidence du Comité d'Etat du secteur de Formosa (Caravela), et

4) réparation et fourniture d'équipement du Secrétariat et de la résidence du Comité d'Etat du secteur de Uno.

Bien que financés de façon très irrégulière, ces projets sont, soit terminés, soit en voie de réalisation. ${ }^{1}$ Le trait le plus frappant de tous ces projets, jugés prioritaires par les instances politico-administratives régionales, est qu'ils n'auront somme toute que peu d'impact concret sur la région. En fait, la prise en compte de ces projets et des listes de propositions de

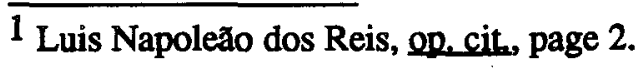


développement issues du G.R.P.-Bolama-Bijagos pour les années 1986, 1987 et 1988, (voir les tableaux 6.4, 6.5 et 6.6) tend à confirmer l'isolement dans lequel évoluent les membres du Parti affectés à des postes administratifs, par rapport à la population en général pour qui l'amélioration des conditions de vie compte assurément plus que le fait, par exemple, de savoir qu'il y a une piscine à Bolama, condamnée de toutes façons, par les nombreuses contraintes techniques propres au pays, à ne fonctionner que sporadiquement.

Pour 1988 (voir Tableau 6.7), la liste des projets est moins importante que celle des années antérieures et ceci s'explique par le fait qu'elle a été tirée de celle des projets jugés prioritaires pour l'exercice du Second Plan Quadriennal. Certaines propositions pour le Second Plan sembleront cependant plus valables en ce qui a trait aux retombées envisageables comme, par exemple, la proposition d'installer un dépôt de carburant à Bolama, la réparation des ports, ou encore, l'acquisition d'embarcations pour le transport entre les îles. Par contre, le rang qu'on leur accorde peut faire craindre que leur réalisation soit quelque peu improbable, du moins par les autorités régionales qui savent très bien différencier les projets qu'elles peuvent elles-mêmes exécuter, de ceux plus facilement acceptables par le financement étranger. D'autres ne sont que la sempiternelle répétition des propositions du G.R.P. pour l'amélioration des infrastructures au service des employés de l'administration. La dernière, quant à elle, n'est qu'une duplication à peu de chose près, des activités du Projet de Développement Agricole Bolama-Bijagos (proposition numéro 15 qui ajoute au projet 
TABLEAU 6.4

Liste des propositions de projets pour 1986

G.R.P.-Bolama-Bijagos

1.Préparation des résidences des présidents des Comités d'Etat de la région et du secteur.

2.Achèvement de l'édifice du comité d'Etat de Bubaque et construction d'une résidence pour fonctionnaires.

3.Construction d'une résidence pour les fonctionnaires à Bolama.

4.Réparation de l'hôpital à Bolama (portes, fenêtres, etc.).

5.Réparation de la maison de la plage d'Ofir.

6.Récupération de l'ancien édifice du Comité d'Etat de São-João.

7.Réparation du Comité d'Etat Régional à Bolama.

8.Achèvement du Comité de Parti de la région à Bolama.

9.Achèvement du nouveau lycée de Bolama.

10.Réparation des écoles primaires.

11.Acquisition d'une vedette rapide pour la "Capitainerie" afin de contrôler la fuite de produits vers l'extérieur et d'assurer le contrôle des ports autour de la ville de Bolama.

12.Construction d'une maison pré-fabriquée pour le responsable de la "Capitainerie" afin de lui permettre un meilleur contrôle du port.

13.Transport terrestre entre Bolama et Bolama de Baixo et São-João et Entchude.

14.Réparation du futur siège du Parti et du Club des Pompiers de Bolama.

Source: PEREIRA, Albino, Mendes, Relatório de actividades, Bolama, 20 de novembro de 1985. 


\section{TABLEAU 6.5}

Liste des propositions de projets pour 1987

G.R.P.-Bolama-Bijagos

1.Achèvement d'une résidence pour les visiteurs à Bolama.

2.Réparation de l'édifice du Comité d'Etat de Bolama.

3.Réparation du "Clube" de Bolama.

4.Réparation et finition du futur siège du Parti à Bolama.

5.Construction de nouvelles résidences pour le président et le secrétaire du secteur de Bubaque.

6.Réparation des rues périphériques de Bolama.

7.Construction de maisons pour fonctionnaires à Bolama.

8.Achèvement de huit maisons pour fonctionnaires à Bubaque.

9.Réparation du cinéma de Bolama.

10.Récupération de la ferme d'Etat de Gâ Moria.

Source: D.P.R.-Bolama-Bijagos, Pasta \#9: Actas, Bolama, 10 de março de 1987. 


\section{TABLEAU 6.6}

Liste des projets prioritaires pour 1988

GRP-Bolama-Bijagos

1.Achèvement du futur siège du Parti à Bolama.

2.Achèvement des huit résidences de Bubaque.

3.Réparation et fourniture d'équipement du secrétariat et de la résidence du

Comité d'Etat du secteur de Formosa.

4. Réparation et fourniture d'équipement du secrétariat et de la résidence du

Comité d'Etat du secteur de Uno.

5.Projet de Chambre Municipale de Bolama.

Source: G.R.P.-Bolama-Bijagos, Lista dos projectos regionais escolhidos pelo Gabinete Regional de Planificacão (GRP) para o Segundo Plano Ouadrienal por executar para o ano de 1988, Bolama, 1988.

agricole l'intervention sur l'ìle de Formosa). Cependant, il peut être en un certain sens notable, le seul fait de la présence de telles propositions qui ne sont certes pas étrangères au fait que les projets régionaux à intégrer au Second Plan ont été préparés par le Cabinet du Ministre Résident de la Province du Sud et non par le seul Cabinet Régional de Planification Bolama-Bijagos. En ce sens, on peut croire que la nouvelle structure provinciale jouit d'un meilleur encadrement et d'une meilleure perception de la nécessité de procéder à des investissements moteurs que les G.R.P.. Par contre, l'orientation même des priorités régionales à réaliser lors du Second Plan Quadriennal ne diffère guère de celle des années précédentes et l'on constate toujours l'emphase portée aux besoins particuliers des 


\section{TABLEAU 6.7}

Liste des projets prioritaires pour le Second Plan Quadriennal

Région Bolama-Bijagos

1.Achèvement d'une résidence pour les visiteurs à Bolama.

2.Réparation du "Clube" de Bolama.

3.Réparation et amélioration du siège de l'administration de secteur de Bubaque.

4.Réparation de l'édifice du secrétariat du Comité d'Etat Régional à Bolama.

5.Réparation des routes périphériques de Bolama.

6.Construction d'habitations pour les fonctionnaires.

7.Réparation et fourniture de matériel pour le cinéma de Bolama.

8.Récupération de la ferme d'Etat de Gâ Moria.

9.Construction de maisons pour les visiteurs à Uno et Formosa.

10.Construction et réparation des ports de Bubaque, Caravela et Uno.

11.Installation d'un dépôt de carburant à Bolama.

12.Réparation de la clinique vétérinaire de Bolama.

13.Installation de centrales électriques à Formosa et Uno.

14.Achèvement du lycée José Marti de Bolama.

15.Projet d'appui aux paysans dans la production de riz, de maraîchage et dans

la fourniture de petit matériel à Bolama, Bubaque, Uno et Formosa.

16.Réparation de l'édifice des douanes de Bolama.

17.Amélioration du port de Bolama.

18.Acquisition d'une embarcation pour permettre le contrôle et la liaison entre les îles.

19.Construction d'une rampe d'accès pour le traversier entre Bolama et SãoJoão.

Source: G.R.P.-Bolama-Bijagos, Lista dos projectos regionais escolhidos pelo Gabinete Regional de Planificacão (GRP) para o Segunde Plano Quadrienal por executar para o ano de 1988. Bolama, 1988. 
fonctionnaires de la ville de Bolama dans le sens de l'amélioration de leur cadre de vie. Il est intéressant de remarquer que l'ensemble des propositions concerne la remise à neuf des édifices et équipements hérités de l'époque coloniale portugaise, et que le discours officiel des responsables politico-administratifs justifie cette orientation en plaidant pour la préservation de ce patrimoine d'une ruine certaine. La situation générale de Bolama et de l'archipel commande cependant une priorisation des besoins dans un tout autre sens afin d'axer les investissements sur des activités plus productives ou, du moins, dont les effets se répercuteront sur l'ensemble de la population.

Cette brève analyse du travail pratique d'un Cabinet Régional de Planification aura permis de constater que ses actions se retrouvent en effet très éloignées de ce qu'elles devraient être pour assurer ou, du moins, favoriser ce que nous entendons par un véritable développement régional. Aux responsabilités de formulation d'un plan régional de développement qui lui incombent, nous avons pu constater que sa perception du plan se limite à l'énumération d'une suite de projets de construction et de réfection d'édifices et de biens publics, sans aucun lien entre eux, si ce n'est qu'ils s'adressent tous aux mêmes bénéficiaires. Par manque de formation dans le domaine, mais aussi sûrement par manque de volonté, aucune stratégie de développement régional ne voit jour aux réunions du G.R.P., que ce soit en formulant des mesures propres à favoriser et développer une prise en charge par la population de ses moyens de production ou encore en recherchant l'amélioration de son bien-être en général. Tout au contraire, semble-t-on se décharger de toute responsabilité de développement des collectivités pour laisser le terrain entièrement libre à d'autres intervenants. En plus de l'absence de préparation de projets populaires en ville ou en campagne, aptes à être financés ou opérés par des organismes externes, le G.R.P. ne 
s'occupe pas non plus d'assurer un rôle de coordination et de concertation des activités régionales des différents ministères et organismes dont les O.N.G.. Il s'en suit qu'il faut formuler d'importantes réserves face au rôle des Cabinets Régionaux de Planification dans le processus de régionalisation du système de planification bissau-guinéen:

"A un processus de planification économique et administratif en théorie décentralisé (v.g. GRP, divisions régionales et sectorielles, comités de base et de tabanca), se substitue en pratique un appareil centralisé où des communications difficiles entre Bissau et le reste du pays, ne font qu'amplifier la coupure ville/campagne. Dans un même ordre d'idées, les GRP, et d'une manière plus générale tout le travail des délégations régionales, sont victimes de la même coupure urbain/rural ou plutôt mode de vie de fonctionnaires urbains versus mode de vie traditionnel. Le processus de décentralisation/régionalisation réalisé en théorie ne fonctionne pas dans la réalité, de par la coupure très nette entre les vues à court terme de ses membres exécutants et décisionnels, et les intérêts à moyen et long terme du développement de la région. La juxtaposition sur ce plan d'effectifs politiques dont les postes ne sont aucunement en jeu et de fonctionnaires ayant une sécurité d'emploi presque tout aussi évidente, n'est pas pour dynamiser le processus de régionalisation mis en branle. Bien que le DPRBolama initie la mise sur pied de GRP sectoriels, la contradiction fondamentale entre les intérêts urbains et les intérêts ruraux reste tout de même présente. Quant à l'élaboration de GRP élargis (où des représentants de la base siégeraient ), elle n'aurait sans doute eu qu'un aspect consultatif, le vrai pouvoir décisionnel revenant de facto à l'autorité suprême régionale, le Président du Comité d'Etat." 1

\subsection{Les orientations de projets}

Quiconque considère objectivement les projets de coopération en place à Bolama, est immédiatement frappé par l'importance que ceux-ci prennent aux yeux de la population. Seules sources d'emploi hormis l'Etat et les quelques commerces de lîle, ces projets doivent bien se garder de créer des effets pervers de l'ordre de l'attentisme tant chez les populations que chez les dirigeants.

1 Alain Rousseau, Rapport final d'activité. Projet d'appui à la Délégation Régionale du Ministère du Plan de Bolama-Bijagos: juillet 1986 à juillet 1988. Bolama, C.E.C.I., juin 1988, pages 7-8. 
En effet il est fréquent de constater que la population, maintes fois, remet son sort entre les mains des projets existants ou éventuels, et qu'ainsi, n'entreprend pas des travaux qui seraient pourtant nécessaires. Elle espère plutôt les voir effectuer par l'assistance d'un projet, comme le prouvent ces quelques villages visités lors de l'enquête de tabanca et qui, depuis la guerre, n'avaient engagé aucun effort de récupération de leurs rizières. Is attendaient un quelconque appui du Ministère du Développement Rural ou d'un projet. Ils ne font d'ailleurs encore qu'attendre à ce niveau. Cette conduite attentiste se traduit concrètement par une baisse de l'attitude responsable de la population face à sa propre prise en main, ce qui avait déjà été constaté par Côté et Demers quelques années plus tôt:

"Par ailleurs, en matière de développement rural, l'île est plus sousdéveloppée que le reste de la région; sa population est plus attentiste, démobilisée et dépendante d'approvisionnements extérieurs. Pourtant le potentiel agricole et piscicole existe et pourrait faire l'objet d'une exploitation efficace répondant aux besoins locaux." 1

Cette attitude de délégation aux projets de tâches essentielles se rencontre aussi chez les autorités politico-administratives et ce, à tous les niveaux. Ainsi, au niveau national, on compte sur l'appui externe pour financer les dépenses de développement. Au niveau régional, la voie est laissée libre à l'aide étrangère pour la réalisation de quelque activité productive que ce soit, puisque les G.R.P. ne s'occupent que du financement et de l'exécution de projets d'amélioration d'infrastructures habituellement urbaines.

Le comportement des autorités régionales dépasse même le cadre de la simple délégation de responsabilités en matière de développement aux projets étrangers, en considérant ceux-ci comme tout autre organe local soumis à leur autorité. Ainsi, le Comité d'Etat n'hésitera pas à tenter de monopoliser, réquisitionner, pour employer le terme local, les

1 Marthe Côté, André Demers, Repères pour un développement nural intégré dans la région de Bolama. Guinée-Bissau, Université Laval, Maîtrise en Aménagement du Territoire et en Développement Régional, novembre 1982, page 209. 
ressources humaines ou matérielles des différents projets pour la satisfaction de besoins particuliers des dirigeants locaux. Par exemple, les projets qui possèdent voitures et bateaux devront constamment composer de diplomatie et de fermeté afin de ne pas se voir affectés à des tâches clairement subordonnées aux besoins particuliers de l'élite locale.

Ces quelques faits qui, notons-le, frisent l'anecdote, n'en demeurent pas moins fondés et constituent certes des facteurs à prendre en considération lors de l'élaboration de tout projet en matière de développement à Bolama.La pratique fera que localement, que ce soit chez la population ou chez ses dirigeants, l'attentisme face aux interventions étrangères sera une attitude omniprésente qui, bien souvent, pourra prendre l'allure d'une entrave sérieuse à une prise en main locale du processus de développement.

D'une manière pratique cependant, si l'on se penche sur les projets étrangers impliqués à Bolama, l'absence de planification tant nationale que régionale qui affecte le système administratif bissau-guinéen, se traduit par la présence, dans une même île, de deux projets ayant des intérêts communs, la pêche, mais semblant partager des points de vue différents. Alors que Pescarte compte développer une pêche que nous qualifierons de professionnelle, axée sur la fourniture d'équipement dispendieux, la formation, le recours massif au crédit, le stockage et la conservation de poisson, l'approvisionnement du marché bolamien tant urbain que rural et, éventuellement l'exportation vers Bissau, lle de Paix, situé de l'autre côté de la rue, escompte élargir ses activités à la pêche traditionnelle, une pêche caractérisée par l'utilisation d'embarcations traditionnelles, d'équipement modeste et l'approvisionnement du marché local. Cette divergence de vue, qui relève de la philosophie même des deux organismes impliqués, l'un, O.N.G., l'autre, entreprise privée, bien que fort compréhensive vue sous cet angle, n'empêche tout de même pas que les conséquences des actions 
entreprises, tant positives que négatives, ne seront à supporter que par la population seule, qui voit son sort être mis en jeu par deux projets bien ancrés dans leurs positions respectives.

On peut éventuellement comprendre l'intérêt pécuniaire qui peut animer l'entreprise privée qui s'est vu octroyer un territoire vierge de toute intervention en matière de pêche et qui, dit-on n'est pas uniquement d'une richesse impressionnante en poissons, mais aussi en crustacés et mollusques. On s'explique moins bien cependant l'implication que compte se donner Ile de Paix dans la pêche traditionnelle, si ce n'est selon une philosophie de préservation et d'exploitation des potentialités propres au mode de vie traditionnel rencontré sur l'̂̀le, en opposition à la philosophie de marché de Pescarte-Bolama. Quoiqu'il en soit, il n'en demeure pas moins qu'lle de Paix, lors de sa première mission de définition de projet en 1984, avait cerné quatre secteurs cibles (pêche, agriculture, santé et approvisionnement en eau) et, qu'avec la venue de Pescarte et du projet agricole du C.E.C.I., ses créneaux originaux d'intervention se sont vus peu à peu diminuer. Pour demeurer en accord avec son orientation habituelle qui est de réaliser "des projets de développement intégré en collaboration avec les populations locales, qui mettent l'accent sur l'autogestion et l'autofinancement éventuel des projets", 1 I'O.N.G. belge se doit, de l'avis même de son coordonnateur à Bolama dans un document destiné au ministère de tutelle du projet, le Ministère du Plan, d'effectuer un certain effort de recul et de distanciation prenant en compte tant la capacité d'absorption en matière d'assistance de la région, que la nature, l'approche et le fonctionnement de l'organisme Iles de Paix: 2

"Discrepâncias na coordenação das intervenções de assistência, ao nivel do arquipélago e na ilha de Bolama, poem em questão a continuação do projecto Ilha de Paz Bolama na direcção prevista (construção de barcos, assistência a pesca).

$\overline{1}$ Martha Jaureguiberry, Ann Lovell, Mission de formulation du projet GBS/85/C01: infrastructures de santé dans la région Bolama-Bijagos, s.1., F.E.N.U./O.P.E., octobre 1985, Annexe XII.

2 Hannes Stegemann, Relatório de actividades da Ilha da Paz de Bolama. 1987, Bolama, Ilha de Paz, 8-12-87. page 31 
Uma duplicação das actividades não se justifica no ponto de vista das instituções e publico europeu que nos financia." 1

Nous ne considérons pas que la construction de bateau ait constitué une des bases de l'intervention d'lle de Paix à Bolama, mais a plutôt été un excellent moyen stratégique de s'impliquer dans la formation d'une équipe de travailleurs. L'abandon de cette phase pour l'immédiat ne dépend pas à notre avis, de la venue de Pescarte, mais plutôt de l'inadaptation des embarcations construites par rapport aux capacités locales et ce, comme il a été établi au troisième chapitre. La remise en cause de l'implication de l'O.N.G. en matière de pêche serait cependant nécessaire et, espérons-le, pourrait être constructive. En attendant, Ile de Paix de Bolama entend s'impliquer plus spécifiquement au niveau urbain ce qui, en soi, est intéressant vu le peu d'intérêt que les interventions étrangères portent généralement au monde urbain. Il peut, d'un côté s'avérer regrettable que l'on ait, semble-t-il, délaissé les domaines de la santé pour l'ensemble de l'île de Bolama, espérant sans doute que la construction projetée par le P.D.I.I.B. d'un centre de santé à Uato Mandinga soit doublée de certaines initiatives en matière de santé. Paradoxalement, l'implication que compte se donner le P.D.I.I.B. dans la mise sur pied d'un projet de fumage de poisson à Bolama de Baixo, ${ }^{2}$ ne semble pas avoir eu d'effets sur les activités de pêche envisagées à Bolama par les deux projets voisins.

Le projet agricole du C.E.C.I., pour sa part, aura consacré ses énergies à la production rizicole et au maraîchage, suivant une optique d'amélioration du niveau de vie des paysans. Il est possible d'affirmer que l'apport apparent du projet sur le développement de l'agriculture a

\footnotetext{
1 Ibid., page 2:

"Des divergences dans la coordination des interventions d'assistance au niveau de l'archipel et de lîle de Bolama, mettent en question la continuité du projet lle de Paix-Bolama suivant la direction prévue (construction de bateaux, assistance à la pêche).

Une duplication des activités ne se justifie pas du point de vue des institutions et du public européen qui nous financent"(traduction de l'auteur).

2 Keil, Jean-Pierre, Avant-projet de développement de l'agriculture dans les îles bijagos, Bubaque, P.D.I.I.B., P.N.U.D., 1988, 8 pages.
} 
été positif. ${ }^{1} \mathrm{La}$ seule ombre au tableau concerne la commercialisation des produits maraîchers produits localement, qui n'a pu bénéficier d'un encadrement basé sur une connaissance approfondie du marché bolamien. En effet, les recherches en la matière ne furent entreprises qu'une fois un nombre imposant de maraîchères impliquées dans la production et, malheureusement, stimulées intensivement par l'appât du gain qu'on leur faisait miroiter. Ainsi leur a-t-on fait réaliser que, compte-tenu du prix élevé des oignons sur le marché local de Bolama, la production et la vente de ceux-ci ne pourraient s'avérer que très lucratives (de 250 à 300 P.G. pour un oignon moyen 2). Bien que 68,7\% des maraîchères de Bolama (57 sur 83) aient acheté des semences d'oignon, ${ }^{3}$ ce qui représente le plus fort pourcentage de semences vendues (tomates $50,6 \%$, chou, $13,2 \%$, poivron, $19,3 \%$, laitue, $20,5 \%$, carotte, $8,4 \%$, gombo, $8,4 \%$ et persil, $1,2 \%), 4$ elles n'ont pu faire augmenter la moyenne quotidienne de vente d'oignons au marché de Bolama, qui est demeuré de l'ordre de un demi à un kilo par jour. ${ }^{5}$ Ainsi, à défaut de pouvoir exporter efficacement vers Bissau leur récolte d'oignons, faute d'encadrement et de planification, de nombreuses maraîchères durent se résoudre à leur réserver un autre usage. Cette expérience, en plus de dénoter l'importance d'assurer un encadrement adéquat à une population somme toute peu férue des pratiques commerciales, confirma la nécessité de voir se spécialiser les actions ultérieures en matière agricole dans des produits déjà connus de la population et bien implantés dans son régime alimentaire (gombo, tomates, laitue):

"Cependant, l'accent mis sur la commercialisation de produits tels l'oignon, le chou, la carotte, l'aubergine et le poivron, ne doit en aucun cas supplanter celui que l'on devrait porter a des produits plus traditionnels et plus prometteurs. En ce

1 Paul Rouillard, Hannes Stegemann, Intégration du projet agricole CECI au projet Iha de Paz, Bolama, C.E.C.I./le de Paix, 20 avril 1988, 13 pages.

2 Brigitte Boies, Alain Rousseau, Enquête sur la commercialisation des produits maraîchers, Bolama, Projet de Développement Agricole Bolama-Bijagos, C.E.C.I., mai 1988, page 61.

3 Ibid., page 17.

4 Ibidem.

5 Ibid., page 73. 
sens, la priorité devrait être accordée aux produits traditionnels même si ceux-ci ne semblent pas, à première vue, très payants; leur force réside cependant dans leur bonne implantation dans les habitudes alimentaires locales. Il importe donc, à notre avis, d'en tenir compte, le consommateur bolamien étant à ce titre celui qui aura le dernier mot." 1

Le projet d'appui à la Délégation Régionale du Ministère du Plan de Bolama sera la seule de ces interventions non reliée directement à une amélioration des conditions de production de lî̀le, pour plutôt constituer une tentative d'appui aux structures régionales de planification. Cette intervention a été rapidement confrontée à l'immobilisme des G.R.P. et à leur manque de vision globale du développement, ce qui lui convia rapidement une fonction reliée à la formation du personnel de la délégation pour la réalisation de son plan de travail.

Initialement prévu comme devant être instigateur d'une véritable dynamique de concertation et de coordination entre les différents intervenants et autorités régionales, ce projet dut composer avec la méfiance certaine et le peu d'empressement des intervenants étrangers confrontés aux moeurs administratives locales selon lesquelles toute tentative de concertation et de coordination doit rénir les différents acteurs politiques et administratifs locaux, que ce soit le Président du Comité d'Etat, le représentant de la police ou encore le responsable des jeunesses du Parti, la J.A.A.C.. Le peu d'implication de la part des autorités régionales dans l'élaboration d'un processus véritable de développement, leur perception de leurs propres intérêts comme étant ceux de la région toute entière, et leur manque d'implication au niveau de la base populaire, ne passent certes pas inaperçus aux intervenants étrangers, qui ne peuvent ainsi que douter de la pertinence à les voir s'immiscer dans la coordination de leurs activités respectives. De plus, la faible crédibilité dont jouissent les membres du Ministère du Plan au sein des Cabinets Régionaux de Planification se rencontre aussi dans l'esprit des intervenants étrangers qui ne voient dans le Ministère du Plan, non

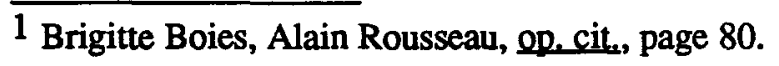


plus que dans SOLIDAMI, la plus récente tentative gouvernementale de mettre au point une structure de concertation, que des organes gouvernementaux de contrôle. Il n'est donc pas étonnant, dans ce cadre, de constater qu'en pratique, les projets vivent souvent dans un magnifique isolement face aux autorités gouvernementales. Sauf lorsque leur collaboration avec les autorités régionales est nécessaire de par les statuts mêmes du projet, les intervenants étrangers ne coopèrent qu'avec le niveau central à Bissau, en ignorant plus ou moins complètement les structures régionales. L'absence de lien de communication efficace entre les ministères à Bissau et leurs délégations régionales, font que les délégués régionaux peuvent être totalement ignorants de ce qu'un projet concernant leur ministère peut entreprendre dans leur région, comme le prouve le témoignage du délégué régional du Ministère de l'Education qui, lors d'un G.R..P, se plaignait de ne posséder aucun détail sur les modalités de construction d'écoles du projet P.D.I.I.B. sur l'île de Uno. ${ }^{1}$

Au niveau de la coordination, dans le cadre des structures actuelles, notons qu'elles est difficile à atteindre:

\begin{abstract}
"Tout d'abord, et il est regrettable de le constater, la majorité des projets ont une méfiance certaine envers les autorités nationales (dont le Ministère du Plan) et n'aiment pas exposer leurs problèmes devant tout le monde. En outre, toute réunion convoquée par le Cabinet Régional de Planification (GRP) doit rassembler les diverses autorités politiques locales, et leur compétence en la matière peut souvent sembler douteuse aux intervenants étrangers. Ce désir d'indépendance et d'autonomie face à l'Etat de la part des projets n'est pas reconnue officiellement; il n'en demeure pas moins présent et s'étend même aux projets entre eux. De plus, l'expérience nous aura maintes fois démontrée que certains projets ont un autonomie toute relative face à leur siège social; la coordination entre les autres projets en est donc difficilement atteignable. La seule solution efficace réside dans de réunions non-officielles (lire sans la présence des autorités nationales) alignant les projets concernés." 2
\end{abstract}

1 Gabinete do Plano da Região de Bolama-Bijagos, Segunda reunião do Gabinete Regional de Plano de Bolama-Bijagos. Bolama, 25 de outubro de 1986, page 2.

2 Alain Rousseau, Rapport final d'activité. Projet d'appui à la Délégation Régionale du Ministère du Plan de Bolama-Bijagos: iuillet 1986 à juillet 1988, op cit., page 6. 
Vu sous cet angle, les besoins tant nécessaires de coordination des activités de développement actuelles et futures, malgré leur urgence afin d'éviter un dédoublement d'activités tel que celui connu à Bolama au niveau de la pêche, semblent difficiles à satisfaire. Il apparaît néanmoins que les structures actuelles (G.R.P. et Ministère du Plan), tout comme les liens entre Bissau et les régions, de même qu'entre les différents ministères à Bissau, se révèlent absolument inadéquates et inopérantes. Faute de voir affecter les activités de coordination et de planification des interventions à venir aux structures régionales et nationales actuelles, tout comme aux seuls projets, il semble de plus en plus évident que le gouvernement bissau-guinéen, s'il désire assurer un développement harmonieux de ses régions, devra mettre de l'avant un organe efficace et disposant de prérogatives certaines en la matière. Cet organe pourrait être SOLIDAMI et il importe donc au gouvernement de le doter des pouvoirs de coordination, de concertation et de planification nécessaires à des activités de développement au niveau des régions, en lui fournissant des liens tant avec les O.N.G. et autres organismes de cooperation au pays, qu'avec les projets sur le terrain et les différents ministères à Bissau comme en région. Dans ce cadre, il conviendra tout autant de définir le rôle des provinces et de les replacer vis-à-vis des régions et de leurs organes.

Retirer les tâches de coordination qui incombent aux G.R.P., revient à les séparer effectivement d'une importante responsabilité liée étroitement à l'élaboration et à l'exécution du plan régional de développement. Cependant, leur inaptitude à définir un plan régional réellement moteur et leur semblant de fonctionnement démocratique et consultatif, démontrent, dans leur forme actuelle, leur inefficacité et, de là, leur inutilité. C'est sans doute suite à un raisonnement semblable que sont apparues les provinces et il faudra se garder de les voir prendre la même avenue que les comités d'Etat et leur G.R.P.. Ceci peut paraître incertain puisqu'entre les régions et les provinces, la seule différence notable en est une de 
niveau et non de contenu, les mêmes personnes se regroupant pour discuter des mêmes problèmes. Seule l'influence du ministre résident pourra éventuellement compenser pour le manque dont font preuve les présidents de régions. L'avenir reste donc à ce stade, le seul garant des succès ou des insuccès des autorités provinciales mais on peut douter qu'elles réussissent, sans l'intervention de changements majeurs, là où les régions ont échoué.

D'une manière générale donc, ce qui différenciera à Bolama les actions des projets financés par l'étranger de celles impliquant les structures politico-administratives régionales, aura été l'omniprésence chez les premiers de leur implication auprès des populations, contrairement aux autorités locales pour qui l'amélioration et la consolidation de leur mode de vie font figure de priorité régionale. De son côté, l'appui à la Délégation Régionale du Ministère du Plan, bien qu'imbriqué au sein des structures officielles régionales, constituait certes une tentative d'organisation des bases nécessaires à la mise sur pied d'un système de planification régional, soit l'organisation d'une méthode permanente de cueillette, la compilation et la diffusion des données socio-économiques régionales et la formation du personnel aux tâches de la fonction. Une fois un minimum de résultats atteints en ce sens, la délégation put alors déborder ce rôle uniquement bureaucratique pour, en conformité avec les orientations nationales, se pencher plus particulièrement sur la concrétisation des aspirations locales d'organisation et de développement des activités de production et de distribution. Encore à ses premiers balbutiements, la Délégation Régionale du Plan a tenté d'initier les premières bases de ce travail d'agent catalyseur des besoins populaires en se faisant relais entre la population et les organismes financiers ou exécutants. Ainsi, suite à l'enquête de tabanca, la délégation à cherché à répondre aux besoins de matériel de pêche artisanale de la population rurale de l'île. Bien que le rôle de la délégation ne soit pas à ce titre d'encadrer l'expérience et que, dans l'éventualité de la découverte d'un financier potentiel, celui-ci peut 
s'avérer plus à même d'assurer le fonctionnement harmonieux du projet, il n'en demeure pas moins que cet organe de l'administration bissau-guinéenne peut veiller au respect des aspirations. locales et de leur désir de préserver leurs structures et leur mode de vie traditionnel, en adressant et en formulant sa demande d'assistance à un organisme qui reconnaît et respecte ces mêmes aspirations. La possibilité des autorités locales de veiller au développement des collectivités locales selon leurs propres aspirations, a été démontrée clairement à ce moment, par le démarrage de négociations d'entente entre Ile de Paix et Pescarte pour la satisfaction par ce premier, des demandes villageoises de petit matériel de pêche, plutôt que par Pescarte qui, de par son orientation vers la pêche professionnelle et l'intégration au sein d'un encadrement rigide des pêcheurs par un système assurant l'écoulement de la production, et une politique de crédit financier impliquant la dépendance financière des pêcheurs envers l'organisme pendant de plus ou moins longues périodes, ne correspondait pas aux volontés villageoises. Il faut constater par cette attitude villageoise, le désir de conserver mode de vie et structures traditionnels le plus possible. In peut sembler évident que les villageois, très imbriqués dans leur culture traditionnelle, entendent d'une certaine façon préserver le plus possible lintégrité de cette culture et de ce mode de vie. De ce fait ils semblent plus enclins à perpétuer la pratique de leurs activités de production traditionnelles où le pêcheur n'est qu'un pêcheur d'appoint, étant avant tout riziculteur et producteur de tout ce que son unité familiale d'exploitation requiert. Il apparaît de plus en plus clairement donc, que cette attitude de refus ou, du moins, de distanciation par certains habitants ruraux, fait montre d'un sentiment, qu'il soit pleinement conscient ou non, de rejet d'un modèle de production qui imposerait une spécialisation plus ou moins importante de leur part dans une seule activité productive afin de rencontrer des responsabilités financières. Par contre, l'exemple des maraîchères qui se seront orientées massivement vers la production 
d'un légume non-traditionnel que la population ne consomme pas ou peu, en l'occurrence l'oignon, représentera par contre le pendant de cette conduite "éclairée".

$\mathrm{Vu}$ sous cette lunette, il est difficile d'ignorer que potentiellement, si l'encadrement et la formation des membres des structures officielles comme le G.R.P. étaient adéquats et que ces structures adoptaient des prises de position plus étroitement liées à la base, celles-ci pourraient s'avérer un instrument de choix dans la concrétisation des aspirations locales, et la coordination et la planification des activités de développement de la région. 


\section{CONCLUSION}

Portrait bolamien

L'étude du milieu socio-économique bolamien a nettement démontré le caractère dual de l'île.

Le monde rural, peuplé de nombreuses ethnies, vit plus ou moins en autarcie, sous un modèle d'économie de subsistance. Son agriculture, sa pêche et ses activités de cueillette ne visent à satisfaire que des besoins simples et limités. En campagne, la vie est encore traditionnelle quoiqu'à des degrés divers selon l'ethnie et l'éloignement de la ville de Bolama. Ainsi peut-on dire que ce mode de vie ancestral se caractérise par une monétarisation des échanges plus ou moins marginale et un niveau de consommation des plus modestes.

De même, la diversité ethnique des habitants de Bolama est remarquable. Cette diversité se traduit par une richesse culturelle évidente. Qui plus est, l'existence d'ethnies régies par une organisation et un mode de fonctionnement de type horizontal, parallèlement à la présence d'ethnies nettement hiérarchisées que nous avons qualifiées de verticales, impliquent l'impossibilité de tirer des généralisations précises pour un monde pourtant de si faible dimension géographique et si peu peuplé. Cette diversité fait apparaître toute une gamme de conduites précises. Chaque ethnie peut être considérée comme spécialiste d'une 
activité particulière, que ce soit la pêche, la culture du riz ou encore le maraîchage ou la cueillette des fruits du palmier. A cette division ethnique du travail se greffe celle des sexes où hommes et femmes possèdent chacun leurs champs d'intervention propre. Changer de spécialisation peut paraître impensable voire même impossible aux yeux de l'individu. Cependant, la collaboration est de mise, qu'elle s'effectue au sein d'une même ethnie dans un même village ou non, entre personnes de sexes différents, d'habilités ou de statuts divers, ou encore entre ethnies différentes. Vu sous cet angle, ce retranchement ethnique et sexuel dans des activités précises nettement arrêtées, loin de constituer une entrave au développement, doit plutôt être considéré comme une particularité à être exploitée. En fait, cette particularité peut aisément devenir une force lorsque l'on considère le professionnalisme souvent mystique qui anime ces peuples dans l'exercice de leurs activités. Parallèlement à cela, il faudra néanmoins garder à l'esprit que la culture de chaque ethnie sera à prendre en compte lorsque l'on considérera l'ouverture de chaque ethnie au changement. Nous avons vu, à ce sujet, que les sociétés verticales, habituées à évoluer dans un cadre précis, plus ou moins rigide, sont plus enclines à accepter l'encadrement nécessaire à la mise sur pied de structures de production et d'échange. Ainsi, les ethnies islamisées se sont démontrées à Bolama, plus ouvertes à la pratique du commerce. Par contre, dans les sociétés où l'enrichissement individuel n'est pas toléré et l'autorité autre que traditionnelle refusée, l'ouverture au changement sera plus limitée. A ce titre, nous n'avons qu'à nous référer aux nombreux essais d'implantation de la traction attelée chez les Bijagos d'autres îles, pour bien saisir l'aspect particulier de cette contrainte. En effet, chez l'ethnie bijago, on considère comme farfelu le fait de vouloir faire travailler un animal, celui-ci n'étant pas fait pour le travail. D'une manière plus globale, l'exemple des Balantes qui ignorent le concept de chef nous oblige aussi à relativiser nos méthodes d'organisation et d'encadrement, tout comme la présence de 
nombreuses cérémonies et le respect du calendrier des activités imposent la souplesse à tout programme d'activité.

La ville de Bolama par contre, affiche un caractère fort différent du reste de l'île. Le salariat et la monétarisation des échanges y sont généralisés et la population qui y habite perd peu à peu contact avec ses racines traditionnelles et les modes de production communautaire et tributaire qui règnent dans le monde traditionnel. Nous constatons qu'à Bolama, outre les services gouvernementaux, les projets de coopération, les commerces et la pêche, les sources d'emploi sont peu nombreuses. Il en découle qu'un fort taux de personnes est constamment à la recherche d'emploi.En fait, en ville, la population est coupée de la terre traditionnellement fournisseuse de nourriture. L'absence d'approvisionnement adéquat du commerce, le prix élevé des biens de consommation et le faible pouvoir d'achat de la population urbaine, la rendent dépendante de l'aide internationale puisque la campagne, qui ignore plus ou moins l'économie marchande, n'engage que très peu de liens commerciaux avec la ville. Il faut par contre se garder de conclure que la monétarisation de l'économie de l'île ne concerne que la ville puisqu'à la campagne, le pouvoir de l'argent n'est pas complètement méconnu de la population. Cependant, jusqu'à tout récemment, le producteur rural ne pouvait d'une part, trouver son compte dans la rétribution de son labeur pour des raisons strictement monétaires, le prix de sa production n'étant pas reconnu à sa juste valeur et, d'autre part, avoir accès à une gamme de produits et services variés. Tout ceci contribue à la dualisation tant économique que humaine de l'île. Cette dualisation ne se traduit pas par l'absence totale de liens entre la ville et la campagne. A ce titre, il est nécessaire de constater que la ville exerce un attrait certain sur les populations rurales comme le démontre l'important exode rural affectant l'île et se concretisant par l'absence pratiquement totale d'hommes dans certains villages. 
L'aspect parasitaire de l'urbain est évident, considéré sous l'angle relatif à la recherche de la satisfaction de ses besoins et à sa monopolisation du pouvoir. La ville de Bolama n'est, pour le milieu environnant, qu'un monde étranger, branché sur l'extérieur, dévoreur des ressources financières du pays. De plus, conjointement avec les autres villes du pays, il est évident que par leur seule présence, ils sont responsables pour une bonne part de la dépendance du pays face aux bailleurs de fonds internationaux et l'aide alimentaire mondiale.

Dans un autre ordre d'idées, il est essentiel de noter que le gouvernement tente d'entraîner la population rurale vers une monétarisation de ses activités courantes. Cette tentative n'est pas d'ailleurs sans trouver l'appui de celle-ci qui, malgré son confinement dans l'autoconsommation et le bas niveau actuel de sa consommation marchande par personne, reste tout de même perméable à l'attrait de l'argent, en autant que les circuits de vente et de distribution lui permettent d'utiliser cet argent pour une consommation variée et intéressante. Ainsi nous retrouvons-nous à devoir composer avec les deux côtés d'une même médaille. D'un côté, nous avons un monde traditionnel où une population rurale, vivant pratiquement en circuit fermé, n'est aucunement encline à produire de surplus dont elle ne saurait que faire, et vaque à ses activités quotidiennes selon un ordre ancestral. De l'autre côté, on note une plus grande ouverture aux innovations suite à l'effet de stimulus qu'exerce la monétarisation de l'économie à travers la généralisation de rapports marchands rendue possible par une restructuration du circuit commercial national et une promotion des activités de production populaire et d'association. 
Le système de planification

Du point de vue administratif, nous avons constaté la décentralisation régionale des divers organes gouvernementaux. Cependant, dans la pratique, la réalité apparaît d'une façon tout à fait différente et l'absence de liens efficaces entre Bissau et les régions pose une entrave de taille au bon fonctionnement de cette décentralisation.

Au niveau local, l'organisation, fondée sur les comités de village, correspond à un effort intéressant de décentralisation, de dynamisation et d'implication de la population dans le processus de développement de la nation. Là encore, dans la pratique, les comités de village semblent paralysés et n'ont pu s'introduire dans la mentalité de la population, nettement plus orientée vers le respect de ses structures traditionnelles de fonctionnement.

Outre la formation des ressources humaines dans les organes gouvernementaux qui est déficiente et les difficultés de communications notoires dans le pays, l'échec de la décentralisation du système bissau-guinéen peut reposer sur la présence d'une entité dominatrice et centralisatrice à tous les niveaux, le Parti. En effet, que ce soit l'Etat ou les comités de village, tous sont infiltrés et suivent la ligne édictée par le Parti. Considérée sous cet angle, l'association Parti-Etat et Parti-structures de base, annihile tout effort dissident de fonctionnement. L'implantation dans les villages de ces structures politiques faut-il le constater en parlant des comités de base, s'est donc avérée un échec malgré l'important potentiel mobilisateur qu'elles possédaient.

En ce qui concerne la planification du développement, la Guinée-Bissau s'est dotée d'un ministère en la matière, le Ministère du Plan, chargé de veiller à l'élaboration et à 
l'exécution de la politique nationale de développement, exposée dans le cadre de plans quadriennaux.

Le volet régional de ce système de planification est assuré par la Délégation Régionale de Planification qui représente le Ministère du Plan en région. La D.P.R. est assistée dans sa tâche par le Cabinet Régional de Planification, organe décisionnel composé des membres des différents ministères en région, et présidé par le gouverneur de la région, le Président du Comité d'Etat Régional, organe administratif régional. Dans la pratique, les activités du cabinet ne se seront pas révélées à la hauteur de la mission de développement et de planification qui lui est attribuée. Le Cabinet Régional de Planification ne sera apparu que sous le jour d'un organe voué à la promotion des intérêts de ses membres, fonctionnaires de l'Etat, sans aucune vision globale du développement régional.

A ce niveau, les propositions formulées par les membres des comités de village lors de l'enquête de "tabanca" sont nettement plus intéressantes. Malgré l'absence chez ceux-ci de connaissances théoriques ou pratiques du développement, il semble que les propositions émanant du milieu soient plus pertinentes en matière de retombées pour les communautés et d'amélioration de leur bien-être en général. On peut clairement à partir de là, dresser le constat que, bien que les comités de villages puissent connaître des problèmes de légitimité face à leurs concitoyens plus proches des structures traditionnelles que de ces organes, il n'en demeure pas moins qu'ils possèdent une excellente connaissance de la réalité villageoise. Par la pertinence de leurs propositions, qui compense largement pour leur manque de formation et d'encadrement ils figurent sur de nomhreux noints. mieux aue les autorités régionales en 
Les nouvelles structures devant peu à peu remplacer les autorités régionales, les cabinets provinciaux, ont encore tout à prouver. Il n'en demeure pas moins qu'ils ont joué un rôle important dans l'élaboration du Second Plan Quadriennal et que leurs propositions en matière de développement régional paraissent plus prometteuses que celles du précédant plan, issues des seuls cabinets régionaux. Il importe à cet égard de souligner les différences entre le Premier et le Second Plan. Ainsi, alors que le Premier Plan a porté l'emphase sur la mise sur pied des conditions nécessaires à assurer un minimum de cohésion de l'appareil économique du pays et que la régionalisation du développement s'est traduite par l'octroi d'enveloppes régionales, le Second Plan, tout en poursuivant les efforts de reconstruction nationale, insiste sur la nécessité d'un certain désengagement de l'Etat. Privatisation de certains secteurs d'activité, libéralisation sans cesse accrue du commerce, et développement des organisations de producteurs et de la production populaire, sont au rang des nouvelles orientations véhiculées par le Second Plan.

Malgré que l'expérience bissau-guinéenne de planification régionale soit apparue comme un échec, nous considérons la planification comme une méthode en soi essentielle:

"L'outil privilégié pour réaliser une stratégie de développement est la planification. Celle-ci est une démarche d'orientation du développement par la détermination de priorités et l'agencement dans le temps de moyens suivant une rationalité idéologique, politique ou pratique spécifique." 1

L'élaboration des deux plans quadriennaux a démontré la faiblesse générale des appareils d'Etat du pays, que les retards dans le dépôt du Second Plan et l'absence de rigueur dans l'application du Premier Plan, ne font que corroborer. Il demeure par ailleurs évident que l'existence d'une philosophie ou,du moins, d'une ligne idéologique bien précise favorisant la recherche de l'autosuffisance alimentaire, l'organisation populaire et, d'une

1 Marthe Côté, André Demers, Repères pour un développement rural intégré dans la région de Bolama. Guinée-Bissau, op. cit, Annexe 5, document préparatoire. 
manière plus générale quoique très lapidaire, l'ambition de créer une société libre de l'exploitation de l'homme par l'homme, constitue un point positif à ne pas négliger, même si la pratique ne peut s'avérer à la hauteur des objectifs tracés. Au niveau central par contre, les analystes remarquent l'existence de trois tendances contradictoires sur bien des points, ${ }^{1}$ ce qui peut porter préjudice au courant de libéralisation amorcé:

- un courant progressiste considérant la libéralisation comme essentielle à la dynamisation de l'économie;

- une branche favorisant le renforcement des structures et du rôle de l'Etat; et,

- l'influence d'organismes internationaux tels la Banque Mondiale et le F.M.I., voulant intégrer la Guinée-Bissau au marché international et veillant à la rationalisation financière de son appareil étatique.

Ainsi donc, officiellement du moins, la Guinée-Bissau ouvre ses portes à la réalisation d'un développement en accord avec les aspirations et la culture de son peuple. Ce refus d'un modèle de développement importé et en désaccord avec les structures traditionnelles, offre la possibilité immense aux intervenants nationaux et étrangers de s'impliquer dans un développement à partir de la base. En contrepartie, la quête incessante de la stabilité financière et de l'équilibre budgétaire, ajoutée à la présence d'organismes internationaux garants de la préservation des liens financiers internationaux, et l'existence d'une contradiction évidente entre la population et ses dirigeants et exécutants membres du Parti, jouent un rôle de contrepoids à ces tendances de développement "au ras du sol". L'accent que porte actuellement le gouvernement, du moins dans ses prises de position officielles, au développement des initiatives locales et à l'exploitation des volontés locales d'organisation, de production et de distribution, a donc imposé une remise en question fondamentale d'un

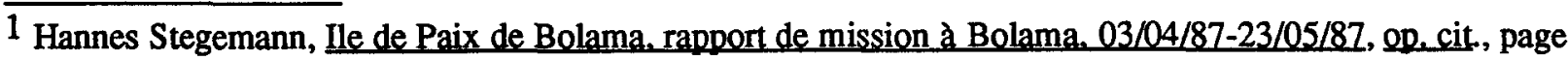
15. 
modèle de développement basé sur la primauté de l'économique en tant que source de progrès social, valeur que véhiculait en pratique le régime de Luis Cabral. En fait, l'étude du milieu bolamien n'aura fait que confirmer que le développement ne peut et ne doit s'effectuer qu'en conformité avec le respect des valeurs locales et la participation de la population. Il devient dès lors évident que le développement n'est pas tant quantitatif que qualitatif:

"Le développement est une capacité généralisée d'une société de se prendre en charge. C'est le processus par lequel cette société devient capable de mobiliser ses forces productives dans la transformation de son milieu en vue d'améliorer ses conditions de vie et le bien-être de ses membres. Il lui faut donc considérer la totalité des aspects de la vie collective: l'environnement, les activités de production et d'échange, les structures de décision, les comportements de consommation, la culture, etc.." 1

Il apparaît clairement que les interventions des projets de coopération à Bolama sont plus en conformité avec cette vision du développement que peuvent l'être les actions et propositions des organes gouvernementaux régionaux. Aucun effort de mobilisation concrète de la population paysanne n'a, par exemple, été mis sur pied par le Ministère du Développement Rural. Le Cabinet Régional non plus ne s'est aucunement impliqué dans des activités concernant la population locale. En fait, analysée sous cet angle, la totalité des interventions des projets de coopération à Bolama s'implique, de près ou de loin, auprès de la population et celle-ci semble répondre positivement par sa participation. On s'aperçoit clairement que les O.N.G. semblent animées par cette vision du développement passant par la participation et l'implication de la population locale et qui présuppose du même coup, la formation en vue de cette population autonome dans la réalisation de ses activités. Le Projet de Développement Agricole Bolama-Bijagos avec son appui à la production de riz, au maraîchage et à la formation d'équipes de vulgarisateurs agricoles, correspond à ce cadre par

1 S.U.C.O.-C.R.A.D., Le développement rural intégré de la région de Bolama, République de Guinée-Bissau, Université Laval, Maîtrise en Aménagement du Territoire et Développement Régional, 1982, Volume I, page 113. 
l'implication qu'il sait donner aux paysans. Le développement de la pêche traditionnelle par Ile de Paix, que l'on entend combiner à un volet de formation des pêcheurs, entre aussi dans cette catégorie. De même la formation de menuisiers et d'artisans est tout aussi conforme à cette vision du développement. Seul le projet de pêche de Pescarte n'adhère pas à cette définition en important une grande quantité de matériel moderne et en tentant d'impliquer les pêcheurs dans les rouages de l'utilisation du crédit et de l'approvisionnement de marchés urbains. La réponse de la population face aux interventions en matière de développement, que nous pouvons considérer autant comme un indicateur de l'acceptation par le milieu d'un projet que comme le fondement de notre conception du développement, est très éloquente. Ainsi la recherche aura nettement démontré que la population préfère et de loin, les petits projets de peu d'envergure financière et technique, lui permettant de satisfaire ses préoccupations quotidiennes, sans amener de bouleversement des structures traditionnelles de fonctionnement et de production, aux interventions et aux projets trop ambitieux. De même, le peu d'implication de la part des autorités gouvernementales dans les communautés villageoises se traduit dans la pratique par le peu de cas que les ruraux portent aux organes officiels et par la méfiance qu'ils éprouvent parfois même envers ceux-ci.

Sur un autre ordre d'idées, le découpage administratif national unissant l'île de Bolama, la section continentale de São-João et l'archipel bijagos pour former une seule région, a démontré clairement qu'il ne correspondait pas à la réalité. La recherche aura entraîné la différenciation et la séparation de lî̀le de Bolama du reste de la région, tant pour des raisons ethniques qu'historiques ou sociales. Il faut souligner à ce sujet, la profonde différence qu'entraîne l'existence, de l'avis de tous les intervenants, à Bolama d'un dynamisme et d'une plus grande ouverture d'esprit aux innovations de la part des différentes ethnies de l'île, 
contrairement à l'ethnie bijago qui reste fortement ancrée dans son mode de pensée traditionnelle et qui reste réfractaire à plusieurs innovations.

Bolama, île à caractère multi-ethnique, apparaît clairement comme ne faisant pas partie de l'archipel bijago. Bolama, capitale administrative régionale, n'a aucun lien avec les différentes îles de l'archipel, tous les réseaux d'échange ou de migration traditionnels unissant l'archipel peuplé presqu'essentiellement de Bijago, à Bubaque plutôt qu'à Bolama où le peuplement, postérieur à celui de l'archipel, s'est effectué par toute une gamme d'ethnies provenant du continent et y important ainsi leurs activités et leur culture traditionnelle. De ce fait, Bolama, de par sa population, compose une entité particulière unique malgré ses liens évidents avec le continent. Tout ceci fait que, pour les fins du mémoire, l'île de Bolama a été considérée comme une région en soi puisqu'après tout, elle possède les particularités propres à toute région, soit de former un espace homogène, distinct et original par rapport aux autres territoires du pays. Ce choix n'aura certes pas été sans faire apparaître de nombreuses contradictions entre la théorie et la pratique, et une certaine ambivalence dans la démarche adoptée. Il ressort clairement que Bolama, en tant que capitale régionale, devrait avoir un certain rôle moteur au sein de la région; il n'en est rien. La réalité politico-administrative bolamienne aura été éloquente sur le réductionnisme pratiqué par les membres des organes gouvernementaux qui, par leur méconnaissance du milieu bijago de l'archipel, par leur chauvinisme ethnique les menant à associer l'ethnie bijago à un simple peuple de cueilleurs et, bien sûr, par leur désir de voir mettre en valeur leur sort d'urbains, ne prendront compte que de leur réalité dans la définition des besoins régionaux. Confronté à ce découpage artificiel où une bonne partie de son destin est liée à des acteurs ne lui appartenant effectivement pas, l'archipel des Bijagos ne peut être que le grand perdant puisqu'il ne possède pas les clés de son développement. 
Le modèle de développement

Considérant les caractéristiques propres au milieu bolamien et les aspirations de la population locale qui n'espère que mieux consolider ses activités courantes et non changer de mode de vie et entrer dans une ère de développement industriel la forçant à se prolétariser, nous privilégierons, à l'instar de nombreux auteurs, un développement endogène. Ce type de développement implique pour une population la prise en compte des ressources tant humaines que matérielles du milieu, et "leur mise en oeuvre et en valeur cohérente",1 et intégré. Ce développement est qualifié d'intégré non seulement parce qu'il intègre une multiplicité de secteurs et qu'il en assure la cohésion, mais parce qu'il émane directement des populations concernées:

"Mais un modèle de développement, même idéal, reste abstrait s'il est défini de l'extérieur et étranger aux préoccupations des populations. Le développement intégré authentique réside moins dans un résultat attendu que dans un ensemble d'activités réalisées selon les attentes des collectivités impliquées, avec leur accord et leur participation active. Ce n'est pas la planification, mais le processus de développement lui-même qui s'intègre aux caractères propres des collectivités locales. Les interventions et projets de développement intégré impliquent donc la participation des collectivités locales à la prise de décision autant qu'à la réalisation des activités de développement." 2

Il faut donc constater qu'à Bolama, le développement ne doit pas se limiter à l'agriculture, mais déborder largement ce cadre pour toucher tant la santé, l'éducation et les communications que la pêche, l'artisanat et la production populaire. Ainsi le développement impose-t-il une remise en question complète de nombreux sujets ayant pu jusque là paraître intouchables. L'éducation par exemple, dans le cadre d'une société africaine impliquée dans

\footnotetext{
1 François Perroux, Pour une philosophie du nouveau développement, Paris, Les Presses de 1'U.N.E.S.C.O., 1981, page 31.

2 S.U.C.O.-C.R.A.D., op. cit., pages 114-115.
} 
son développement devrait-elle se contenter de former de futurs fonctionnaires imbus de valeurs intellectuelles et culturelles typiquement occidentales ou des individus polyvalents connaissant les techniques pratiques relatives à l'agriculture, à la pêche, à la santé ou encore à l'organisation populaire de la production? Il en est de même de la nécessaire remise en question du calendrier scolaire aucunement en accord avec le calendrier agricole qui nécessite la présence des jeunes à plusieurs moments de l'année.

L'importance d'assurer la concordance entre les actions entreprises ou proposées et les aspirations locales doit être garante d'un développement à la mesure et à l'écoute du milieu. On comprendra que sous cet angle, toute initiative d'investissement financier et matériel massif à Bolama, de par sa disproportion, n'apparaîtra guère viable. L'exemple de Pescarte peut paraître à ce titre évocateur avec son budget de 6,5 millions de dollars américains pour une période de cinq ans et demi. La méfiance des populations rurales face à cette affaire de gros sous se conçoit facilement lorsque l'on considère le fait que tout crédit important implique un enchaînement nécessaire face au financier se traduisant ici, comme nous l'avons précédemment noté, par une dépendance évidente au système de Pescarte. Ceci démontre en outre l'attachement des populations rurales à leurs modes de production traditionnels, cellesci n'étant pas prêtes à les abandonner et à se consacrer à un travail régulier les coupant de leurs liens avec la terre.

Cette implication fondamentale entre les activités à développer et la population, met au premier plan la nécessité d'existence de circuits entre la base et les organes exécutifs du développement. Au niveau politique, le lien théorique unissant les structures supérieures du Parti et donc de l'Etat, aux structures de base, les comités de village, de quartier et de base, n'a pas de contrepartie dans la pratique car les G.R.P. et les orientations générales prises par les comités d'Etat régionaux, les agents nationaux du développement, démontrent plutôt le 
contraire. Il devient donc évident, comme le texte du mémoire le souligne à maintes reprises, qu'un réajustement des structures politico-administratives est nécessaire dans un sens d'un meilleur flux de la base vers le sommet et d'un effort plus notable de coordination, de concertation et de planification des activités des divers intervenants socio-économiques. Qui plus est, l'implication de la population dans son propre développement permettra d'éviter l'apparition de mentalités d'assistés sociaux où la population n'a plus de dynamisme et remet son sort entièrement entre les mains de tiers. A ce propos, les populations rurales ont semblé moins défaitistes et plus aptes à prendre en main et à assurer leur développement que les populations urbaines. Ainsi, dans le cadre de l'enquête de village, on remarque que les comités des quartiers urbains de Bolama font état d'une moindre implication en matière de développement, à croire qu'il n'y a rien à améliorer en ces lieux. A titre d'exemple, seulement deux propositions émanent des comités en ville, soit l'aménagement d'un puits et une proposition de traction animale, alors qu'à la campagne, les propositions abondent. L'explication de cette différence entre rural et urbain peut soit s'expliquer par la moins bonne implication populaire des comités en ville qu'en campagne, soit par un certain désintéressement vis-à-vis des activités productives de la part des citadins. Dans un cas comme dans l'autre, cela pourrait indiquer une attitude plus attentiste de la part du milieu urbain.

Le respect de la voix populaire en matière de développement permettra seul d'assurer un développement harmonieux utilisant les potentialités et les modes de fonctionnement traditionnels de ces sociétés. Notre perception du développement, trop souvent dépendante de contraintes temporelles, est en opposition directe avec les moeurs locales. L'échec relatif de plusieurs expériences en matière d'appui au développement trouve sa cause dans la trop grande attention que nous portons au facteur temps et, les projets, toujours soumis dans leur 
calendrier et leur budget à des contraintes temporelles, n'effectuent bien souvent que les premiers balbutiements nécessaires à la mise en place du processus. Leur retrait provoque l'abandon des opérations et, à la longue, la démobilisation d'une population de plus en plus blasée de voir se succéder une suite de projets qui, calqués sur le rythme de réalisation occidental, n'ont à peine le temps de s'implanter dans l'esprit des Africains habitués à vivre depuis des millénaires au rythme de la succession des saisons et des périodes de croissance de la végétation. A Bolama, Pescarte prévoyait pouvoir assurer une pêche autonome et rentable en deça de 18 mois, temps que le projet a effectivement nécessité pour se tailler un semblant de réputation, avec le résultat qu'il s'est vu contraint de façon plus réaliste de prolonger le projet de quatre ans. Le projet de développement agricole du C.E.C.I., faute de financement, se retrouve dans un processus hâtif et prématuré de désengagement, à la grande déception des intervenants du projets et de la population qui sait que les résultats ne faisaient que commencer à apparaître.

Cette particularité de lenteur relative dans le changements chez les sociétés traditionnelles que l'on devient à même de mesurer lorsque l'on engage un développement par la base, constitue une contrainte majeure qu'ignore trop souvent l'intervenant étranger, tout comme son fournisseur financier, trop pressés de voir se concrétiser en l'espace de quelques mois les fruits du travail. En Afrique et à Bolama en l'occurrence, l'acceptation du changement par une collectivité ne peut passer que par un processus lent de discussion et de réflexion, où la dynamique de fonctionnement traditionnel de la société entre en scène et impose ses vues de prudence, perceptibles dans de longues négociations. ${ }^{1}$ Tout projet qui

1 Abdul Gadri Djalo, Introduction de technologies appropriées en vue d'augmenter la production de l'huile de palme dans la région de Bolama-Bijagos (Guinée-Bissau), Université Laval, Travail du cours E.R.U.-13 338, Planification de Programmes, 1986, page 16;

Hugues Dupriez, Paysans d'Afrique Noire, Dakar, E.N.D.A., Editions Terres et Vie, 1982, pages 46-47; et, S.U.C.O.-C.R.A.D., op.cit., page 115. 
oeuvrera à Bolama et tout le travail relatif à la planification du développement devront faire leur cette contrainte temporelle ne permettant pas l'obtention de résultats rapides, probants et immédiats, mais demeurant tout de même essentielle et fondamentale au développement intégré. A ce niveau, le succès du processus dépendra tant des capacités de l'interlocuteur local, comité de village ou conseil traditionnel, que de celle de l'intervenant, national ou étranger, public ou privé, tous conscients des limites et contraintes qui les touchent, dont le fait que la palabre ne prend pas l'allure d'une assemblée démocratique au sens où on l'entend, où chacun exprime librement son opinion:

"Il convient également de remarquer que les individus qui interviennent dans la palabre expriment les groupes dont ils émanent et les forces sociales que ces groupes représentent. Le consensus auquel la palabre conduit, cette unanimité qui a impressionné tant d'animateurs communautaires, le consensus n'est pas fondé sur les individus, mais sur l'équilibre des forces sociales. En ce sens, le consensus traditionnel est très différent du consensus moderne parce qu'il ne résulte pas de délibérations, mais de cet équilibre de forces inégales. Dans ces conditions, respecter l'équilibre comme déclare le vouloir l'animation rurale, c'est conserver l'organisation traditionnelle et ses inégalités. Une telle idée se trouve aussi à la base du développement communautaire et de son souci d'éviter toute tension (une tension étant le résultat d'un déséquilibre). On comprend dès lors que ces approches participationnistes aient été accusées de travailler au maintien du statu quo."1

Ce processus de participation en accord avec les structures locales de la population n'est pas le seul à être en quelque sorte calqué sur la réalité africaine. Ainsi, face aux conditions de vie particulièrement rustres de Bolama et à l'impossibilité d'y adapter de technologies modernes de production, tant d'organisation et de gestion de la production que de technologie en soi, il faut comprendre la nécessité de baser toute action sur de petits projets de production populaire, peu consommateurs d'energie et d'outillages complexes, au risque même de se voir accuser d'affubler ces populations d'un développement arriéré. Il paraîtra inopportun de se demander pour le moment, à l'exemple de Hans Geisslhoeer, si, en orientant le

1 Albert Meister, La participation pour le développement. Paris, Editions Economie et Humanisme, Les Editions Ouvrières, 1977, pages 49-50. 
développement du pays sur le monde rural, on ne tente pas ainsi de persuader le pays à "renoncer à l'industrialisation et à la construction d'une société moderne". ${ }^{1}$ De même ne doiton pas chercher à savoir si le recours, par la production populaire, à une technologie peu consommatrice de capital et peu innovatrice technologiquement parlant, ne "fixe" pas le sousdéveloppement et la misère en limitant "la quantité de produits disponibles pour la population, donc le bien-être social" et en ne fournissant pas à la population un "raccourci technologique" en la tenant éloignée des techniques de production plus avancées et aussi plus performantes. ${ }^{2}$

En fait, pour le moment présent, il nous semble évident que Bolama ne peut trouver son compte que dans ce type de développement:

"La stratégie de développement proposée pour la région exclut, à court et à moyen terme, l'option d'un développement axé sur l'implantation d'industries de transformation, notamment de produits agricoles. D'une part les données sociologiques, écologiques et économiques évoquées précédemment permettent de comprendre la difficulté pratique d'une telle option et son impact globalement négatif sur le développement des populations; d'autre part, il serait bien mal indiqué de concentrer les efforts sur un type de développement qui répondrait à des besoins de consommation externes sans avoir au préalable solutionné les problèmes relatifs à la production des principaux aliments de base de la région. L'option industrielle créerait peu d'emplois, diminuerait la main-d'oeuvre agricole active dans la production traditionnelle et rendrait le travail dépendant de l'extérieur." 3

A l'instar de Côté et Demers nous voyons le développement de Bolama dans la recherche de la satisfaction des besoins réels de consommation de la population et de l'amélioration de leur bien-être en général. ${ }^{4}$ In nous semble nécessaire, pour ce faire, d'augmenter la production locale et ce, que ce soit dans l'agriculture, la pêche ou les

1 Hans Geisslhofer, Planification villageoise en Guinée-Bissau; problèmes-expériences-perspectives, Dakar, E.N.D.A., Série Etudes et Recherches, No. 70-81, octobre 1981, page 5.

2 Arghiri, Emmanuel; Celso, Furtado; Hartmut, Elsenhans; Technologie appropriée ou technologie sousdéveloppée?, Paris, P.U.F., Collection Perspective Multinationale, 1981, page 5.

3 Marthe Côté, André Demers, Repères pour un développement rural intégré dans la région de Bolama. Guinée-Bissau, op. cit., page 208.

4 Ibid, pages 208-209. 
productions artisanales telles celles du savon, du sel, de l'huile de palme, etc., ou de la transformation de la production comme par exemple le développement des techniques de conservation des aliments. Cette amélioration tant quantitative que qualitative de la production locale doit mener la population à dépasser le simple niveau de la subsistance, pour ainsi lui permettre une certaine accumulation. De même est-il nécessaire de réviser les structures politico-administratives locales dans le sens d'une meilleure prise en charge de ces appareils par la population et de viser l'amélioration des circuits d'échange. ${ }^{1}$ Anne-Marie Hochet insiste d'ailleurs sur le fait que l'on doive avant tout tenir compte des dimensions humaines et matérielles du pays pour nous amener à prioriser les initiatives assimilables et réalisables par les cellules économiques de base (unités familiales d'exploitation, groupements de producteurs, villages, etc.), en accord avec l'exploitation des ressources existantes et une plus grande responsabilisation populaire face au modèle poursuivi. ${ }^{2}$ De cette manière, "situer correctement le développement [...] à la base, ce n'est pas en rabaisser les ambitions, c'est seulement lui donner le moyen d'exister et d'aller plus loin".3

L'amélioration des structures d'appui au développement doit toucher aussi bien les structures de base que les structures régionales dans le sens déjà indiqué et, d'une manière générale, toute cette démarche nécessitera le recours à l'organisation des paysans, producteurs divers et population en général dans tout un système d'associations et de regroupements, lesquels ne doivent pas servir de moyen de contrôle de la part de l'Etat ou encore correspondre à des modèles associatifs étrangers aucunement en accord avec la réalité locale, comme nous l'enseigne l'expérience coopérative sénégalaise:

1 S.U.C.O.-C.R.A.D., Volume I, op.cit., pages 116-117.

2 Anne-Marie Hochet, Paysanneries en attente: Guinée-Bissau. Dakar, Environnement Africain, Série Etudes et Recherches, No. 79-80, janvier-février 1983, page 174.

3 Ibidem. 
"Issus de l'extérieur, la structure et les statuts de la coopérative ne sont pas adaptés au contexte socio-économique du milieu. Les zones d'incertitude de cette nouvelle organisation sont rapidement contrôlées par l'élite locale qui détient les ressources et économiques et techniques du village. Elle contrôle l'information, limitant ainsi l'accessibilité de la masse aux organes décisionnels de la coopérative. Cette situation transpose l'organigramme social au sein de la coopérative, ce qui permet à l'élite locale de contrôler la destinée des paysans sur le plan social et organisationnel." 1

Nouvelle orientation du régime, l'octroi aux populations du libre droit d'association peut devenir l'axe central de l'effort national de développement socio-économique, du moins auprès des intervenants et participants impliqués au niveau de la production et de la distribution. Les modalités d'association (notamment les modalités légales) restent cependant à définir et l'on ne peut douter que seule la pratique permettra d'évaluer la portée de cette ouverture de l'Etat face à l'initiative collective. Pour Bolama, confiné à l'isolement qu'entraîne son statut insulaire, il ne fait de doute que cette orientation privilégiant les actions de la base pourrait lui permettre peu à peu d'améliorer son sort et de s'intégrer à l'effort national entrepris en ce sens. Seule l'union des efforts urbains et ruraux de développement, combinée à une interaction réciproque de ces deux mondes où chacun pourra jouir de la place qui lui revient, exempt des préjugés souvent véhiculés par une majorité d'intervenants à propos du caractère marginal et inutile de la ville, grâce à une réorientation de celle-ci vers la production populaire artisanale et le soutien des activités rurales, pourra porter fruit. Ainsi, l'île de Bolama pourrait intégrer sa campagne et sa ville dans un effort commun de prise en charge de son destin, en conformité avec les besoins réels de sa population, de sa culture et de ses aspirations.

1 Jacques Gingras, Les coopératives au Sénégal; bilan et perspectives, s.l., C.E.C.I., avril 1986, page 21. 


\section{BIBLIOGRAPHIE}

\section{Ouvrages généraux}

\subsection{Publications officielles}

Araujo Lima, Renaldo (de). Elementos para uma programação deso trabalhos do sistema de planificacão regional, 1981/1982. Bissau, Ministério da Coordenação Económica e do Plano, Departamento de Desenvolvimento Regional, 1981. 12 pages.

Banco Nacional da Guiné-Bissau. Câmbios. Bissau, 4/05/87.

Banco Nacional da Guiné-Bissau. Câmbios. Bissau, 1/02/88.

Baraï, Teté, João. Diagnóstico do sector de Bolama. Bolama, D.P.R.-Bolama-Bijagos, julho de 1986. 25 pages.

C.E.C.E.P.. Assistance au Commissariat d'Etat à la Coordination Economique et au Plan: document de projet. s.1., décembre 1979. 23 pages.

Comité de Estado do Sector de Bolama. Recenseamento de 1987. Bolama, 1987.

D.D.R.. Circulaire. s.l., mars 1985. 1 page.

D.D.R.. Desenvolvimento regional: Programa Bienal de Desenvolvimento 1980/1981. Bissau, Commissariado de Estado da Coordenação Económica e Plano, maio de 1980. 9 pages.

D.D.R.. Relatório da missão do Secretário de Estado as regiōes de Tombali. Bolama-Bijagos e Quínara, de 17 a 23 de setembro de 1982. Bissau, Secretaria de Estado do Plano e Cooperação Internacional, setembro de 1982.9 pages. 
D.D.R.-Bolama-Bijagos. Preparacão do Plano Quadrienal 1983-1986; diagnóstico da região administrativa de Bolama-Bijagos. Bolama, Ministerio da Coordenação Economica e Plano, maio de 1982. 61 pages.

Departamento de Informatica. Tabela salarial 1988. Bissau, 1988. 1 page.

D.P.R.. Lista nominal das seccões por sectores administrativos. Bissau, 25 de fevereiro de 1987. 1 page.

D.P.R.. Proposition de projet d'appui à la DPR-MICEPCI (Divisão de Planeamento Regional): République de Guinée-Bissau. Bissau, Novembre 1985.19 pages.

D.P.R.-Bolama-Bijagos. Colecção estatística. Bolama, 1988. 1 page.

D.P.R.-Bolama-Bijagos. Diagnostico da região de Bolama-Bijagos. Bolama, 23 de agosto de 1986. 13 pages.

D.P.R.-Bolama-Bijagos. Inquérito de tabanca. Bolama, avril 1987.

D.P.R.-Bolama-Bijagos. Inquérito dos projectos. Bolama, 9 de janeiro de 1988.

D.P.R.-Bolama-Bijagos. Pasta \#9;Actas. Bolama, s.d..

D.P.R.-Bolama-Bijagos. Pasta \#16: FNI. Bolama, s.d..

Encyclopaedia Britannica. 1987. Britannica Book of the Year. Chicago, Encyclopaedia Britannica Inc., 1987. page 660.

Gabinete de Planificação da Região de Bolama-Bijagos. Proposta de projectos a serem executados durante o período do Plano na Região de Bolama-Bijagos. Bolama, D.P.R.-Bolama-Bijagos, 12 de outubro de 1983. 1 page.

Gabinete do Ministro Residente. Projecto de Câmara Municipal, Bolama. Bolama, Provincia do Sul, março 1988. 15 pages.

Gabinete do Plano da Região de Bolama-Bijagos. Acta da primeira reunião do Gabinete Regional do Plano de Bolama-Bijagos. Bolama, Ministerio do Plano, 10 de março de 1987. 4 pages.

Gabinete do Plano da Região de Bolama-Bijagos. Acta da reunião. Bolama, Ministerio do Plano, 1982. 1 page.

Gabinete do Plano da Região de Bolama-Bijagos. Aviso, ordem do dia. Bolama, D.P.R.Bolama-Bijagos, 26 de fevereiro de 1987. 1 page.

Gabinete do Plano da Região de Bolama-Bijagos. Lista dos projectos regionais escolhidos pelo Gabinete Regional de Planificação (GRP) para o Segundo Plano Quadrienal, a serem financiados pelo orcamento do investimento (F.N.I.) para o desenvolvimento da região de Bolama-Bijagos. Bolama, D.P.R.-Bolama-Bijagos, 4-1-88. 2 pages. 
Gabinete do Plano da Região de Bolama-Bijagos. Lista dos projectos regionais escolhidos pelo Gabinete Regional de Planificação (GRP) para o Segundo Plano Ouadrienal por executar para o ano de 1988. Bolama, D.P.R.-Bolama-Bijagos, 1988. 1 page.

Gabinete do Plano da Região de Bolama-Bijagos. Propostas de projectos para o $2^{\circ} \mathrm{Plano}$ Quadrienal de 1988. Bolama, D.P.R.-Bolama-Bijagos, 4 de janeiro de 1988. 1 page.

Gabinete do Plano da Região de Bolama-Bijagos. Relacão identificativo dos projectos a serem executados anualmente na Região de Bolama-Bijagos. Bolama, D.P.R.-BolamaBijagos, 13 de março de 1987. 1 page.

Gabinete do Plano da Região de Bolama-Bijagos. Relatório. Bolama, Ministerio do Plano, 1987. 5 pages.

Gabinete do Plano da Região de Bolama-Bijagos. Segunda reunião do Gabinete Regional do Plano de Bolama-Bijagos. Bissau, 25 de outubro de 1986. 3 pages.

Gabinete Regional de Planificação da Região de Bolama-Bijagos. Acta de reunião tida neste Gabinete. Bolama, D.P.R.-Bolama-Bijagos, 13 de outubro de 1982. 1 page.

Gabinete Regional de Planificação da Região de Bolama-Bijagos. Estatutos do Gabinete Regional de Planificacão de Bolama-Bijagos. Bolama, D.P.R.-Bolama-Bijagos, 5 de outubro de 1982. 3 pages.

Gabinete Regional de Planificação de Bolama-Bijagos. Projecto a apresentar a organização CECI para o seu possível financiamento. Bolama, D.P.R.-Bolama-Bijagos, 2 de julho de 1984. 1 page.

Gabinete Regional de Planificação de Bolama-Bijagos. Acta da reunião. Bolaam, D.P.R.Bolama-Bijagos, 28 de setembro de 1985. 5 pages.

Guinée-Bissau. Conférence des Organisations Non Gouvernementales: document d'orientation. Bissau, 7-10 novembre 1985.68 pages.

Hochet, Anne-Marie. Etude des habitudes de consommation et des besoins en produits d'importation des populations rurales de Guinée-Bissau. Bissau, MICEPCI, mars 1981. pages 117-123.

Hochet, Anne-Marie; Uldely, Seco. Les ex-royaumes Pepel du Tor et du Biombo: zones d'émigration temporaire: situation socio-économique.Guinée-Bissau, Commissariat à la Coordination Economique et au Plan, novembre 1980. 46 pages.

International Monetary Fund. Yearbook 1988. Washington D.C., Direction of Trade Statistics, 1988, page 209.

MICEPCI. Plano Quadrienal de Desenvolvimento Económico e Social 1983/1986; Plano anual 1985. Republica da Guiné-Bissau, s.d.. 56 pages + annexes. 
MICEPCI. Primeiro Plano Ouadrienal de Desenvolvimento Económico e Social 1983-1986. Bissau, Grupo de Trabalho Sectorial (Agricultura, Pecúaria e Florestas), abril de 1982. A: Relatório da situacão actual, 45 pages.

MICEPCI. Primeiro Plano de Desenvolvimento Económico e Social 1983-1986. Bissau, Grupo de Trabalho Sectorial (Agricultura, Pecúaria e Florestas), setembro de 1982. B: Relatório de estrategia e objectivos, meios necessários e programação, pages 47-104.

Ministère de la Coordination Economique, du Plan et de la Coopération Internationale. La production populaire en Guinée-Bissau. Bissau, en collaboration avec OXFAMBelgique, septembre 1985. 37 pages.

Ministère de la Main-d'Oeuvre et de la Sécurité du Revenu. Concepts et éléments d'analyse du marché du travail. s.l., Service des Etudes Régionales et de l'Analyse Conjoncturelle, Direction de la Recherche, janvier 1985. 104 pages.

Ministère du Plan. Estimation du déficit céréalier en 1988 et requête d'assistance introduite auprès de la communauté internationale. Bissau, décembre 1987.5 pages.

Ministério da Coordenação Económica, Plano e Cooperação Internacional. Linhas directrizes e grandes orientacões do II Plano de Desenvolvimento Económico e Social. Bissau, 20 de setembro de 1985.41 pages.

Ministério da Coordenação Económica e Plano. Recenseamento Geral da Populaçao e Habitacao, 16 de abril de 1979: resultados provisorios (fase II). Bissau, Departamento Central de Recenseamento, fevereiro 1982. 241 pages + annexes.

Ministério da Coordenação Económica e Plano. Recenseamento geral a população e da habitação, 16 de abril 1979. Bissau, Departamento Central de Recenseamento, fevereiro 1982. Volume II Lista nominativa das localidades recenseadas, 93 pages.

Ministério do Desenvolvimento Rural e Pescas. Anuário estatístico 1985. Republica da Guiné-Bissau, Gabinete de Planeamento, abril de 1986. 69 pages.

Ministério do Plano. Quarta reunião do Conselho Consultivo do Ministério do Plano: primeira fase do II Plano Ouadrienal. Republica da Guiné-Bissau, fevereiro de 1988. 16 pages.

Nations-Unies. Annuaire statistique du commerce international. 1981. New York, Publication des Nations-Unies, 1983. Volume I, Commerce par pays, 1301 pages.

Organisation des Nations-Unies pour l'Alimentation et l'Agriculture. Assistance d'urgence pour l'archipel de Bijagos en vue de la relance de la production vivrière: conclusions et recommandations du projet. Rome, OSRO/GBS/101, 1983. 37 pages.

P.A.I.G.C.. Teses para o IV Congresso do PAIGC. Bissau, Edição do Departamento de Informação, Propaganda e Cultura do Comite Central do P.A.I.G.C., julho 1986. 40 pages. 
P.A.M.. Développement rural à buts multiples. Rome, WFP/CFA:17/13-A(WPMA), 1984. 19 pages.

Pereira, Albino, Mendes. Relatório de actividades. Bolama, 20 de novembro de 1985.1 page.

Província do Sul. Acta: primeira reunião do Conselho Directivo Provincial Alargado aos responsaveis regionais. Bolama, novembro de 1986.13 pages.

Reis, Luis, Napoleão (dos). Comités do Partido da Região de Bolama-Bijagos, Comités do Partido das seccōes de Bolama. Bolama, D.P.R.-Bolama-Bijagos, 6 de dezembro de 1986. 9 pages.

Reis, Luis, Napoleão (dos). Relatório da Delegacia do Plano da região de Bolama-Bijagos do ano de 1987, Bolama, D.P.R.-Bolama-Bijagos, 1987. 5 pages.

République de la Guinée-Bissau. Texte présenté par la République de la Guinée-Bissau à la Conférence des Pays les Moins Avancés. s.l.n.d., pages 23-77.

Rousseau, Alain. Notas sobre o inquérito de tabanca para a ilha de Bolama e a secção de São-João. Bolama, D.P.R.-Bolama-Bijagos, outubro de 1987. 16 pages + annexes.

SCET-AGRI. Etude des prix et incitations aux producteurs ruraux. République de GuinéeBissau, MICEPCI, janvier 1986. 154 pages.

Secretaria de Estado da Presidência para os Assuntos Economicos e Cooperação Internacional. Guine-Bissau; sintese da situação económico-financeira e perspectivas de médio prazo. Bissau, fevereiro de 1987.30 pages + annexes.

Secrétariat d'Etat à la Présidence pour les Affaires Economiques et la Coopération Internationale. Etude préliminaire sur l'amélioration de l'organisation et du fonctionnement de SOLIDAMI, Document de travail. s.l., $1^{\circ}$ juin 1988, version No. 2. 13 pages.

S.E.P.C.I.. Primeiro Plano Quadrienal de Desenvolvimento Economico e Social 1983-1986. Bissau, s.d.. Volume 1, Relatorio Geral, 205 pages.

Serviço Meteorologico Nacional. Estação Meteorologica de Bolama. Republica da GuinéBissau, s.d.. 1 page.

Socomin. Serviço de contrôlo. Bissau, 15/01/80. 1 page. 


\subsection{Livres}

Andrade, Mario (de). Amilcar Cabral; essai de biographie politique. Paris, Librairie François Maspero, 1980. 169 pages.

Cabral, Amilcar. Unité et lutte. Paris, Petite Collection Maspero, 1980. 329 pages.

Carreira, António. A etnonímia dos povos de entre o Gâmbia e o estuário do Geba. Bissau, 1964. pages 233-275.

C.E.C.I.. Guinée-Bissau, dossier pays, CECI. s.1., Programme de formation, décembre 1984. 84 pages.

Côté, Marthe; Demers, André. Repères pour un développement rural intégré dans la région de Bolama, Guinée-Bissau. Université Laval, Maîtrise en Aménagement du Territoire et en Développement Régional, novembre 1982. 284 pages.

Duarte, Fausto. E assim nasceram cidades e vilas. s.1., Imprensa Nacional da Guiné, 1946. pages 41-57.

Duquette, Danielle, Gallois. Dynamique de l'art bidjogo (Guinée-Bissau); contribution à une anthropologie de l'art des sociétés africaines. Lisbonne, Instituto de Investigação Científica Tropical, 1983.261 pages.

Geisslhofer, Hans. Planification villageoise en Guinée-Bissau; problèmes-expériencesperspectives. Dakar, E.N.D.A., No. 70-81, octobre 1981. 25 pages. (Coll. Série Etudes et Recherches).

Groupement Lavalin/Delcanda. Etude générale sur les transports. s.l. Banque Africaine de Développement, juin 1980. volume I, Base socio-économique.

Handem, Diana, Lima. Nature et fonctionnement du pouvoir chez les Balanta Brassa. Bissau, I.N.E.P., 1986. 271 pages.

Hochet, Anne-Marie. Paysanneries en attente: Guinée-Bissau. Dakar, Environnement Africain, No. 79-80, janvier-février 1983. 174 pages. (Série Etudes et Recherches).

L'État du monde, édition 1991, annuaire économique et géopolitique mondial. Éditions La Découverte, Editions du Boréal, Montréal, 1990. 631 pages.

Lopes, Carlos. A transição histórica na Guiné-Bissau; do movimento de libertação nacional ao Estado. Genève, Mémoire présenté pour le Diplôme de Recherche en Etudes du Développement, Institut Universitaire d'Etudes du Développement, novembre 1982. 393 pages.

Lopes, Carlos. "Ethnie, Etat et rapports de pouvoir en Guinée-Bissau", Itinéraires, notes et travaux, No. 22, mars 1982, Première Edition, Genève, 117 pages.

Pierson-Mathy, Paulette. La naissance de l'Etat par la guerre de libération nationale; le cas de la Guinée-Bissau. Paris, U.N.E.S.C.O., 1980. 155 pages. 
Republica da Guiné-Bissau. Potentialités agricoles, forestières et pastorales. s.l., SCETInternational, 1978. Volume I.

Rudebeck, Lars. Guinea-Bissau. A Study of Political Mobilization. Uppsala, The Scandinavian Institute of African Studies, 1974. 277 pages.

Rudebeck, Lars. Guinea-Bissau: difficulties and possibilities of socialist orientation.Uppsala University, Institute of Political Science ans the Working Group for the Study of Development Strategies (A.K.U.T.), january 1978. 37 pages.

Rudebeck, Lars. "Problème de pouvoir populaire et de développement", Research Report, No. 63, 1982, chapitre IV, Le coup d'Etat militaire du 14 novembre 1980, cité dans, C.E.C.I. Guinée-Bissau, dossier pays, CECI. s.l., Programme de formation, décembre 1984. pages 52-56.

Santos Lima, A. J.. Organizacão económica e social dos Bijagos. Lisboa, Centro de Estudos da Guiné Portuguesa, No. 2, Soc. Ind. de Tipografia, 1947. 143 pages.

Sarmento, Isabel. Análise do sub-sistema de planeamento e da administracão regional da Republica da Guiné-Bissau. s.l., 1985. 137 pages.

S.U.C.O.. La pensée polique d'Amilcar Cabral. Dossier S.U.C.O. Montréal, Secteur Education, mars 1977.76 pages.

S.U.C.O.-C.R.A.D.. Le développement rural intégré de la région de Bolama. République de Guinée-Bissau. Université Laval, Maîtrise en Aménagement du Territoire et Développement Régional, 1982. Volume I, 287 pages.

S.U.C.O.-C.R.A.D.. Le développement rural intégré de la région de Bolama. République de Guinée-Bissau. Université Laval, Maîtrise en Aménagement du Territoire et Développement Régional, 1982. Volume II, 329 pages.

S.U.C.O.-C.R.A.D.. Le développement rural intégré de la région de Bolama, République de Guinée-Bissau. Université Laval, Maîtrise en Aménagement du Territoire et Développement Régional, 1982. Volume III, Carte d'occupation des sols, 54 pages.

1.3 Revues, périodiques et journaux

Benoit, J. R. (de). "La Guinée-Bissau: le démarrage du nouvel Etat", Afrique Contemporaine, No. 83, janvier-février 1976, pages 11-17.

Cardoso, Carlos; Ribeiro, Carlos, Rui. "Considerações sobre as estruturas socioeconómicas das sociedades agrárias e a sua evolução histórica; um estudo de caso", Soranda: Revista de Estudos Guineenses, Bissau, I.N.E.P., No. 3, janeiro 1987, pages 5-20. 
Dianoux, H. J.. "La Guinée-Bissau et les îles du Cap-Vert",Afrique Contemporaine, No. 107, janvier-février 1976, pages 1-6.

Felkai, Istvan. "Guinée-Bissau; tenir la promesse faite aux paysans ...", Le Monde Diplomatique, avril 1983, pages 13-15.

Grupo Dinamizador. Produção Popular: Boletim de Informação. No. 1, março de 1987, 19 pages.

Hair, P. E. H.."Beaver on Bulama", Boletim Cultural da Guiné Portuguesa, No. 58, an XV, Bissau, 1960, pages 360-383.

Lepri, Jean-Pierre. "Contribução para a análise sociológica da Guiné-Bissau actual", Soranda; Revista de Estudos Guineenses, No 1, Janeiro 1986, pages 143-168.

Lopes, Carlos. "A Guiné-Bissau a procura de um modelo social", Soranda; Revista de Estudos Guineenses, No. 1, janeiro 1986, pages 5-38.

Republica da Guiné-Bissau. Boletim Oficial, No. 1, 4 de janeiro de 1975, No. 28, 12 de julho de 1975, No. 9, 28 de fevereiro de 1976,No. 48, quarta-feira, $1^{\circ}$ de dezembro de 1976, No. 5, 29 de janeiro de 1977, No. 8, 19 de feveiro de 1977, No. 40, 8 de outubro de 1979,No. 6, 6 de fevereiro de 1982, No. 27, 11 de julho de 1986, No. 32, quarta-feira, 13 de agosto de 1986, No. 14, 4 de abril de 1987, No. 13, 4 de maio de 1987.

Santos, Americo, Ramos (dos). "Estabilização e subdesenvolvimento: lições da GuinéBissau", Economia e Socialismo, No. 60, janeiro-março de 1984, pages 45-58.

Santos Lima, A. J.. "Os Bijagos e o regime do matriarcado", Boletim Cultural da Guiné Portuguesa, Lisboa, vol. II, No. 7, julho, 1947, pages 593-617.

1.4 Documents de projets

Albert, Hugues; Blais, Serge; Côté, Marthe; Thiran, Pierre. Projet de développement agricole dans les îles Bolama-Bijagos, République de Guinée-Bissau. s.l., Programme Etudes et Projets de Développement Rural, C.E.C.I., novembre 1984. Tome I, Etude de base, proposition de projet, 138 pages.

Bitard, Jacques. Appui à l'agriculture dans les îles Bijagos et développement des movens de production agricole. s.1., F.E.N.U., GBS/85/CO3, avril 1987. 208 pages.

Boies, Brigitte; Rousseau, Alain. Enquête sur la commercialisation des produits maraîchers. Bolama, Projet de Développement Agricole Bolama-Bijagos, C.E.C.I., mai 1988. 80 pages. 
Boies, Brigitte; Rousseau, Alain. Guide pour l'analyse des systèmes de production; archipel Bolama-Bijagos, Guinée-Bissau. Bolama, Projet de Développement Agricole BolamaBijagos, C.E.C.I., mai 1988. 26 pages + Annexes.

Braconier, Philippe (de); Drory, Elizabeth. Rapport de mission de juin 1985: prétude de l'Ile de Paix de Bolama. Huy, Iles de Paix, 1985. 40 pages + Annexes.

Braconier, Philippe (de); Drory, Elizabeth. Rapport de mission en Guinée-Bissau du 3 au 13 avril 1987. Huy, lles de Paix, 12 mai 1987. 4 pages.

Brunet, Yves; Thede, Nancy. Rapport de mission d'étude sur le projet d'étude pour la mise en place de structures régionales de développement (Guinée-Bissau). Montréal, le 5-779. 22 pages + annexes.

F.E.N.U.. Construction d'infrastructures scolaires et mise en place d'un nouveau programme d'enseignement en milieu rural dans les îles Bijagos; accord de projet. s.l., GBS/85/CO2, 1985. 27 pages + annexes.

Fernandes, Raul, Mendes. "Nhomincas e Bidjogos, da pesca de "subsistência" a pesca "comercial"", Soranda, Revista de Estudos Guineenses, No. 4, julho 1987, Bissau, I.N.E.P., pages 58-94.

Gonzales, Juan, E.; Lamy, Etienne. Projet de développement agricole dans les îles de Bolama-Bijagos, République de Guinée-Bissau; Evaluation an II. Programmation an III. s.l., Programme Etudes et Projets de Développement Rural, C.E.C.I., janvier 1988. 31 pages + annexes.

Harris, V.. Guinée-Bisssau: développement intégré des îles Biajgos: rapport de mission inter-agences. New York, Nations-Unies, avril 1982. 58 pages + annexes.

Jaureguiberry, Martha; Lovell, Ann. Mission de formulation du projet GBS/85/C01: infrastructures de santé dans la région Bolama-Bijagos. s.l., F.E.N.U./O.P.E., octobre 1985.88 pages + annexes.

Keil, Jean-Pierre. Avant-projet de développement de l'agriculture dans les îles Bijagos. Bubaque, P.D.I.I.B., P.N.U.D., 1988. 8 pages.

Landing, Savane. BNA-Guinée-Bissau, rapport final, s.1., 1983. 129 pages.

Lovell, Anne. République de Guinée-Bissau, formulation du projet GBS/85/CO1. Infrastructures de santé dans les îles Bijagos, troisième partie. s.l., Fonds d'Equipement des Nations-Unies (F.E.N.U.), Bureau pour l'Exécution des Projets (B.E.P.), août 1985.95 pages + annexes.

Ministério dos Negocios Estrangeiros da Républica Italiana. Sintese do Projecto Centro de Pesca Artesanal em Bolama, Guiné-Bissau. s.l., Ittica Valdagri, Serviço Especial Intervenções Extraordinárias nos Países em vias de Desenvolvimento, s.d.. 29 pages. 
Payeur, Simone. Enquête en milieu rural, 1983, document No. 1. s.l., Projet de Développement Rural Zone 2, Service Evaluation, avril 1983. 104 pages.

Payeur, Simone. La riziculture de bas-fonds en Guinée-Bissau: contraintes et possibilités de développement dans le cadre du projet de développement rural de la Zone II. Montpellier, Centre National d'Etudes Agronomiques pour les Régions Chaudes, Ecole Supérieure d'Agronomie Tropicale, janvier 1982. 104 pages.

Pescarte. Captura por espécie referente aos meses de janeiro, fevereiro e marce 1988. Bolama, Secretaria de Estado das Pescas, 12 de abril de 1988.

Pescarte. Relação nominal por pescadores com canoa à motor. Bolama, Secretaria de Estado das Pescas, 12 de abril de 1988.

P.D.I.I.B..Budget couvrant la contribution PNUD (en dollars Etats-Unis); Développement Intégré des Iles Bijagos. s.l.n.d., 2 pages.

P.D.I.I.B..Budget couvrant la contribution FENU (en \$ EU), 1986-1988: Construction d'infrastructures scolaires et mise en place d'un nouveau programme d'enseignement en milieu rural dans les îles Bijagos. s.l.n.d.. 2 pages.

Rouillard, Paul. Programmation du projet CECI-Bolama-Bijagos (octobre 86 à septembre 87). Bolama, C.E.C.I.-Bolama-Bijagos, 1986.7 pages.

Rouillard, Paul. Réflexions sur le projet Bolama-Bijagos. Bolama, Projet de Développement Agricole Bolama-Bijagos, C.E.C.I., août 1987. 33 pages.

Rouillard, Paul; Stegemann, Hannes. Intégration du projet agricole CECI au projet Tha de Paz. Bolama, C.E.C.I./lle de Paix, 20 avril 1988. 13 pages.

Rousseau, Alain. Rapport final d'activité, Projet d'appui à la Délégation Régionale du Ministère du Plan de Bolama-Bijagos: juillet 1986 à juillet 1988. Bolama, C.E.C.I., juin 1988. 13 pages + Annexes.

Simard, Carole. Rapport d'activités. Bissau, C.E.C.I., 1986. 30 pages + annexes.

Stegemann, Hannes. De de Paix de Bolama, rapport de mission à Bolama, 03/04/8723/05/87. Huy, lles de Paix, 1987. 53 pages.

Stegemann, Hannes. Relatorio de actividades da Tha da Paz de Bolama, 1987. Bolama, Itha de Paz, 8-12-87. 3 pages.

Thiran, Pierre. Caractéristiques du crédit agricole du projet de développement agricole Bolama-Bijagos du MDR-CECI. Montréal, EPDR-CECI, s.d.. 2 pages.

Thiran, Pierre. Projet de Développement Agricole dans les Iles de Bolama-Bijagos, République de Guinée-Bissau: rapport d'activités, janvier-février-mars 1986. s.1., C.E.C.I., Programme Etudes et Projets de Développement Rural, mai 1986. 43 pages + annexes. 


\subsection{Documents photocopiés}

Brodeur, Jean. Guinée-Bissau. s.l., texte polycopié, septembre 1979. 5 pages.

Djalo, Abdul Gadri. Introduction de technologies appropriées en vue d'augmenter la production de l'huile de palme dans la région de Bolama-Bijagos (Guinée-Bissau). Université Laval, Travail du cours E.R.U.-13 338, Planification de Programmes, 1986. 23 pages.

François, Pierre. La Guinée-Bissau après le coup d'Etat de novembre 1980. s.l., mai 1981. 28 pages.

Henry, Christine. Hommes seuls et femmes volages, note sur le mariage des Bijagos de Caravela. s.l., texte non-publié, juin 1984, 28 pages.

Hochet, Anne-Marie. La situation économique en milieu rural dans le secteur de Bolama, Note de travail. Bissau, le 22-2-81, 14 pages.

\subsection{Entrevues}

Entrevue avec Alfredo Medina, Secrétaire du Comité d'Etat Régional, Bolama, février 1988.

Entrevue avec deux membres du comité de tabanca du village de Madina, Colly Baldo et Bubal Baldo, le 11 novembre 1987.

Entrevue avec El Hadj Camara, directeur pour la région Bolama-Bijagos des Armazens do Povo, Bolama, 30 mai 1988.

Entrevue avec Luis Napoleão dos Reis, délégué régional, D.P.R.-Bolama-Bijagos, Bolama, le 25 mai 1988.

Entrevue avec Paul Rouillard, chef du Projet de Développement Agricole Bolama-Bijagos, Bolama, le 2 juin 1988.

Entrevue avec Philippe de Braconier, secrétaire général d'Tles de Paix, Bolama, 10 avril 1987.

Entrevue avec Stefano Moretti, coordonnateur de Ittica Valdagri à Bolama, le 25 février 1988.

Entrevue avec Tina Stegemann, anthropologue, Bolama, lles de Paix, $1^{\circ}$ juin 1988. 


\subsection{Cartes}

Institut Géographique National-France. Guinée-Bissau. 1: 500 000. Paris, 1981. couleur.

Junta das Missões Geográficas e de Investigações do Ultramar. Bolamạ. 1: 50 000. Lisboa, Ministério do Ultramar, 1952. couleur.

SCET-International. Guinée-Bissau, Zone 4: Région de Bolama. 1: 500 000. s.1., 1978. Volume III.

2. Ouvrages complémentaires

2.1 Publications officielles

Commission Economique pour l'Afrique. Stratégies, priorités et programmes pour les pays africains les moins développés. Addis-Abéba, Nations-unies, Conseil Economique et Social, Quinzième Session/Sixième Réunion de la Conférence des Ministres, 9-12 avril 1980. 8 pages.

\subsection{Livres}

Albertini, J.-M.. Les mécanismes du sous-développement. Paris, Les Editions Ouvrières, 1967. 349 pages.

Amin, Samir. Classe et nation dans l'histoire et la crise contemporaine. Paris, Les Editions de Minuit, 1979. 263 pages.

Amin, Samir. L'Afrique de l'Ouest bloquée: l'économie politique de la colonisation 18801970. Paris, Les Editions de Minuit, 1971. 322 pages.

Amin, Samir. Le développement inégal; essai sur les formes sociales du capitalisme périphérique. Paris, Les Editions de Minuit, 1973. 365 pages.

Badouin, Robert. Economie rurale. Paris, Librairie Armand Colin, 1971. 598 pages.

Beauvoir, Simone (de). Le deuxième sexe. s.1., Editions Gallimard, 1949. Tome 1, 510 pages. 
C.E.C.I. Rapport annuel 1988-89. Montreal, C.E.C.I., juin 1989. 20 pages.

Delfaud, Pierre; Lacour, Claude; Lajugie, Joseph. Espace régional et aménagement du territoire. Paris, Précis Dalloz, 1979. 884 pages.

Diouf, Makhtar. Economie Politique. Dakar, Nouvelles Editions Africaines, 1981. Tome 2: Théorie économique néoclassique et marxiste, 254 pages.

Dumont, René. Paysanneries aux abois: Ceylan. Tunisie, Sénégal. Paris, Editions du Seuil, 1972. 253 pages.

Dupriez, Hugues. Paysans d'Afrique Noire. Dakar, E.N.D.A., 1982, 256 pages. (Editions Terres et Vie).

Emmanuel, Arghiri; Furtado, Celso; Elsenhans, Hartmut. Technologie approprié ou technologie sous-développée?. Paris, P.U.F., 1981. 189 pages. (Collection Perspective Multinationale).

Freire, Paulo. Lettres à la Guinée-Bissau sur l'alphabétisation; une expérience en cours de réalisation. Paris, François Maspero, 1978. 179 pages. (Coll. Cahiers Libres 343).

Gagnon, Gabriel. Coopératives ou autogestion; Sénégal. Cuba. Tunisie. Montréal, Les Presses de l'Université de Montréal, 1976. 482 pages.

Gingras, Jacques. Les coopératives au Sénégal: bilan et perspectives. s.l., C.E.C.I., avril 1986. 60 pages.

Lacoste, Yves. Contre les anti-tiers-mondistes et contre certains tiers-mondistes. Paris, Editions La Découverte, 1985. 143 pages.

Lénine. La maladie infantile du communisme (le "gauchisme"). Pékin, Editions en Langues Etrangères, 1970.130 pages.

Lénine. Que faire?, s.l., Editions du Seuil, 1966. 319 pages.

Meillassoux, Claude. Terrains et théories. Paris, Editions Anthropos, 1977. 344 pages.

Meister, Albert. La participation pour le développement. Editions Economie et Humanisme, Les Editions Ouvrières, Paris, 1977. 176 pages.

Mende, Tibor. De l'aide à la recolonisation. Paris, Editions du Seuil, 1975. 315 pages.

Moreira, J. M.. Os Fulas da Guiné Portuguesa na panorâmica geral do mundo Fula. Bissau, 1964. pages 289-327 et 417-432.

Perroux, François. Pour une philosophie du nouveau développement. Paris, Les Presses de I'U.N.E.S.C.O., 1981. 279 pages.

Staline J.. Des principes du léninisme: conférences faites à l'Université Sverdlov. Pékin, Editions en Langues Etrangères, 1970. 123 pages. 
Touraine, Alain. Les sociétés dépendantes: essais sur l'Amérique Latine. Paris, Duculot, 1976. 266 pages.

\subsection{Revues, périodiques et journaux}

Bugnicourt, Jacques. "Illusions et réalités de la région et du développement régional en Afrique", Revue Tiers-Monde, Tome XIX, No. 73, janvier-mars 1978, pages 109138.

Campbell, Bonnie. "Eléments d'analyse pour l'étude comparée des sociétés dépendantes: Afrique, Amérique latine", Revue Tiers-monde, Tome XVIII, No. 72, octobredécembre 1977, pages 737-762.

Colett, Adrian. "L'autorité dans les communautés rurales africaines au Sud du Sahara", Le Courrier Afrique Caraibes Pacifique Communauté Européenne, No. 99, septembreoctobre 1986, pages 69-71.

Dumont, René. "La Guinée-Bissau peut encore échapper au sous-développement", $\underline{\text { Le }}$ Monde Diplomatique, juillet 1979, pages 8-11.

Hugon, P.; Sudrie, O.. "La crise de la planification africaine, diagnostic et remèdes", Revue Tiers-Monde, Tome XXVIII, No. 110, avril-juin 1987, pages 407-434.

Jacquemot, Pierre. "Crise et renouveau de la planification du développement", Revue TiersMonde, Tome XXV, No. 98, avril-juin 1984, pages 245-268.

La Presse. Montréal, samedi le 30 septembre 1989.

Progrès-Dimanche. Chicoutimi, $1^{\circ}$ octobre 1989, Projets reliés au Tiers-Monde; le dernier budget Wilson fait mal,

Sicault, Dr G.. "Le développement "au ras du sol"", Revue Tiers-Monde, Tome XVIII, no. 72, octobre-décembre 1977, pages 699-708. 
ANNEXE

QUESTIONNAIRE DE LENQUETE DE VILLAGE 
MINISTERIO DA COORDINACRO ECONONICA PILNO E COOPERACRO INTERNACIONAI DEPARTATENTO DE DESENVOLVITENTO REG IONAI

\section{FICHA DE TLBINCS}

REGIZO DE SECTOR TABANCA

NORE DO INQUIRIDOR

DATA DO PREENCHIVIENTO

PRESIDENTS DO COITTE DE BISE

VICE-PRESIDENTE DO COIITTE DE BASE

$$
\begin{aligned}
& I=\text { POPUIAGRO } \\
& \text { I.I - II } \\
& \text { I.2 - F } \\
& \text { I.3 - IF I.4 - Nímero de Fómilias }
\end{aligned}
$$

I.5 - Etnia preponderantc

I.6 - Etnia secundiria

\section{II - EDUCACIOO}

$2 . I$ - Nesta Tabanca há cscola $\square$ sim $\quad$ in 2.2 - Quantas

2.3 - Ate que classe se leciona

2.4 - Se nio há escola nesta tabanca as crianças frequentam a Escola de a. Kuis.

2.5 - Durante o ano lectivo de 198,1989 a Escola

Ficou fechada $\left\{\begin{array}{l}\text { sim } \square \\ \square \text { no } \square\end{array}\right.$

Qunntos meses

Causas.... $\left\{\begin{array}{l}\text { Falta de professor } \\ \text { Falta de alumos } \square \\ \text { Falte de material } \\ \text { Outras } \square\end{array}\right.$

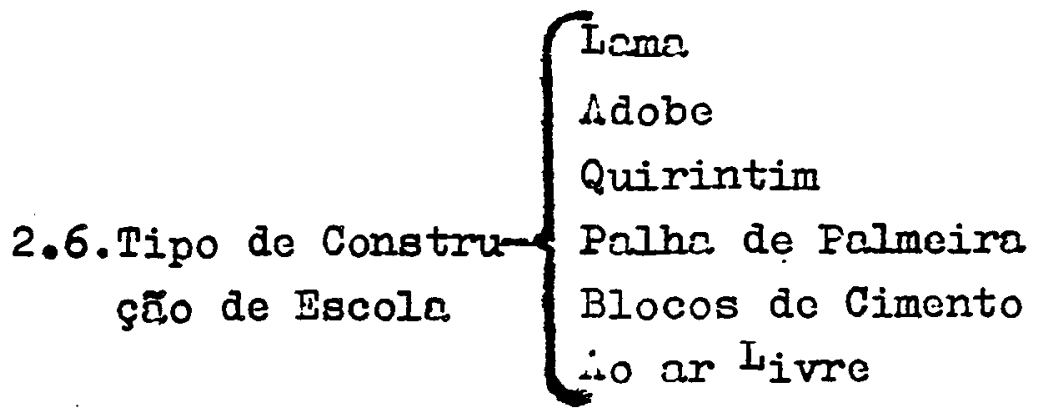


2.7 En que cstado se encontre Bom Estrodo

Precise de repercȩões $\square$ No tecto INas parede JNo piso

2.8 Qunntos alunos frequentrm c. escoln desta tobence (II

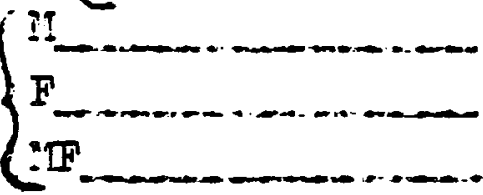

2.9 Qunntos Professorcs

2.10 Hб escola Corinica $\left\{\begin{array}{l}\square \text { sim } \\ \square \text { ñ̃o }\end{array}\right.$

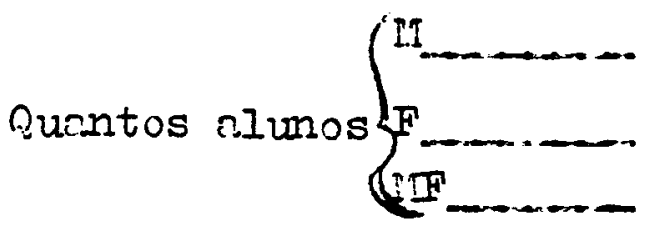

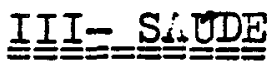

3.1 HE. Posto Saniterio $\left\{\begin{array}{l}\square \text { sin } \\ \square \text { n:o }\end{array}\right.$

3.2 Hé Frnaḱcic.

3. 3 HE. Bnformoiros
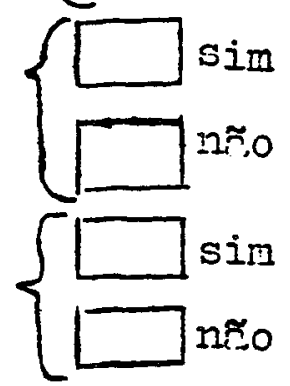

3.4 Qunntos

3.5 Ho Djambacós

3.60 abastecimento de medicamentos

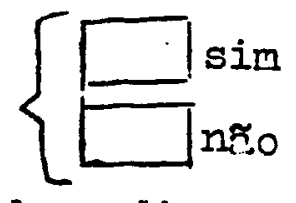

ไ.

3.7 So ñ̃o houvor Fosto Sctitério noste tabence deve-se ir a.te c. KIS prre fazer tratamento.

\section{$\underline{\underline{I V}}=\mathrm{COIUUII}=\mathrm{IACNO}$}

4.1 ARDN \& houvide, $\left\{\begin{array}{l}\square \text { sim } \\ \square \text { ño }\end{array}\right.$

4.2 Há Rádios

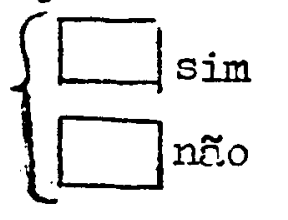

$4 \cdot 3$ Qucntos 


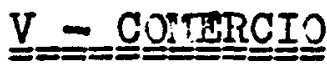

5.1 Ammazóns do Povo $\square$ sim

5.2 Socomin $\left\{\begin{array}{l}\square \text { sim } \\ \square \text { ño }\end{array}\right.$

5.3 Lojas Privades

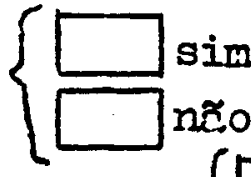

sim

5.4 Quentes

5.5 Se he lojas olas vendas
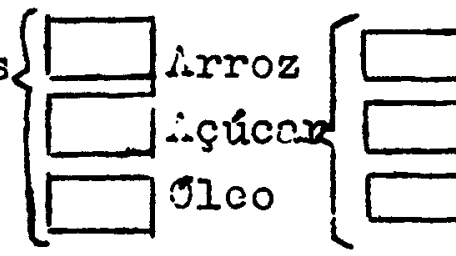

Petróleo

Sil

Tabnco

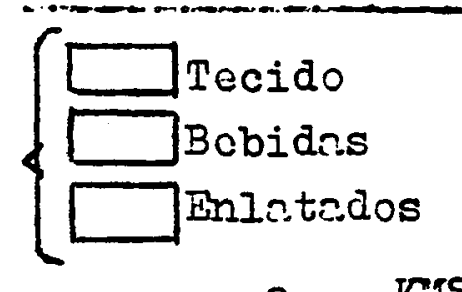

5.6 Se wo he lojns na trbance a populaç⿰彳 vai ate 2 KT!S. para fazer compras e vonder sous principais produtos.

5.7 Há foiras neste tabonca

5.80 tipo de foir

5.90 tipo de construgko

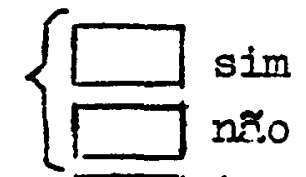

$\{$ :0 or livre

\{ Coberto

$\left\{\begin{array}{l}\text { definitivo } \square \\ \text { provisoris: } \square\end{array}\right.$

5.10 Principnis Produtos vondidos no feire deste trebenca ou ne de

No tompo seco a)

No tempo chuva n) b)

b) c)

c) a)

d).

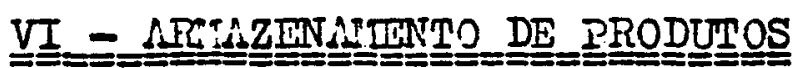

6.I So houver lojns ou celeiros colcctivos nosta tebnnce o ismez.e feito:

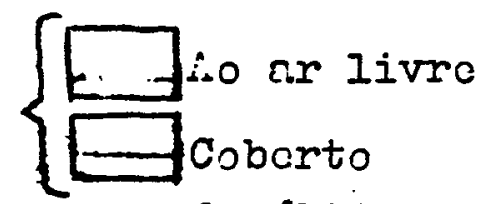

$6.2 \Lambda$ capacidade do rmazensmento dn tiltime campanha foi de:

- Armazóns do Fovo

- Socomin

- Comerciantos Privedos

- Colciros Colectivon

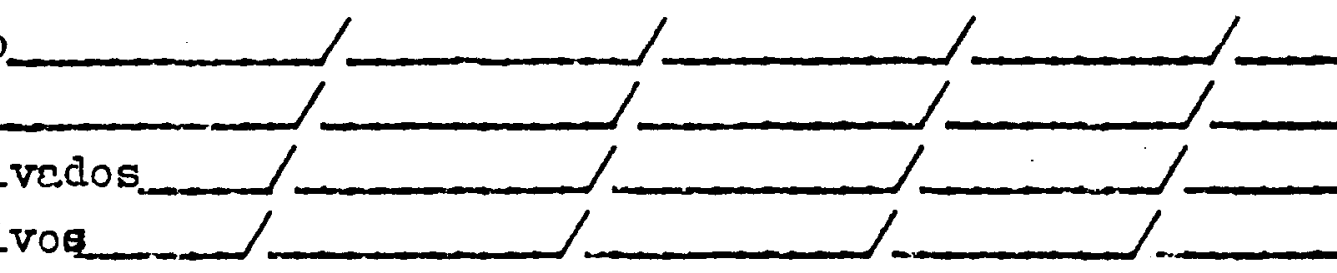

KGS KGS. KGS KGS.

6.3 icdidas dos drmezóns Cobcrtos sto:

- Comprimentos

- Irrgura.

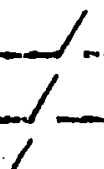

- situra. 
6.4 Os prociutos mais importantes desta tabanca nå são comercializados cevicio a falta de:

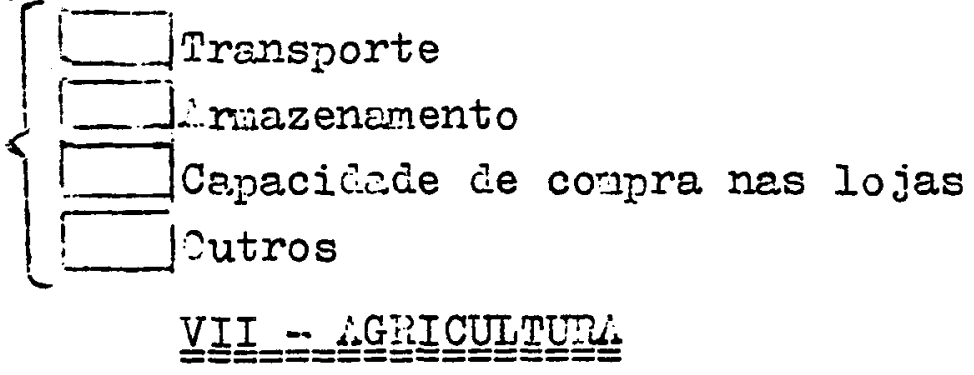

7.1 Ouais os principais proiutos comercializados desta tabanca?
a)
b)
c)
d).
e).
f)
g).
h)
i)

7.2 ?uais os cultivos(secuncários?)

a)

d)

7.3 Há apanhadores de chabéu

7.3 Ha apanhadores de chabeu

7.5 Utiliza-se a tracção aninal na agricultura

b)

e) c)

f)

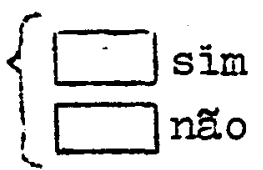

7.5 Utilize-se a tracção aninal na agricultura

7.4 Quantos

7.6 Quantas: Charruas. Carroças.

7.7 Existem bolanhas e campos de cultivo abencionados

7.8 is causas de abandono foram?

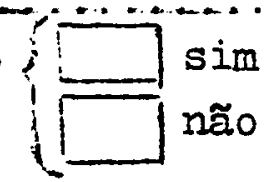

$\left\{\begin{array}{l}\square \\ \square \\ \square\end{array}\right.$ Guerra กลัo

7.9 Há gado bovino $\left\{\begin{array}{l}\square \text { sim } \\ \square \text { ñ̃o }\end{array}\right.$ Seca udança de Iessoal Invasão de água salgada Jutras

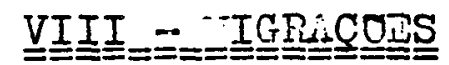

8.I Há trabalhaciores da tabanca que vão trabalhar:

No Senegal

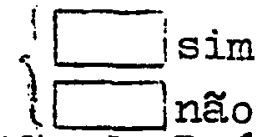

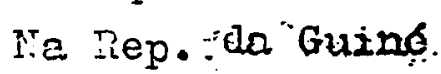

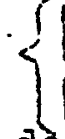

do mês

de. ao mês cie

ço mês cie ao mês de

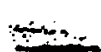

do mês de ao mes de. co mês de. ao mês de 


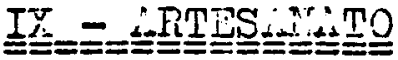

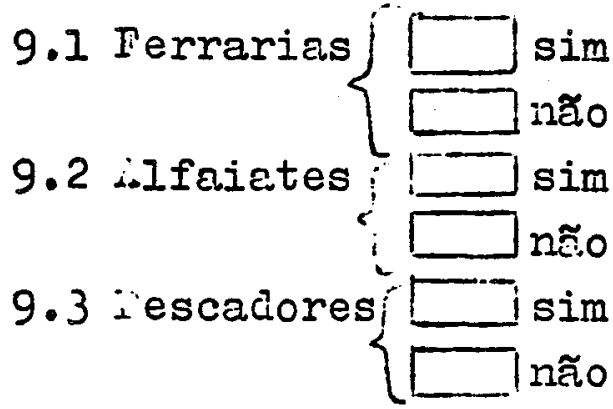

9.4 Construtores de Casas

9.5 Tecilo $\left\{\begin{array}{l}\square \text { sim } \\ \text { não }\end{array}\right.$

9.5 Vinho de a.Ima

9.7 Cerŝnica …......

9.80 Ieo de ralma

9.9 Sal

9.10 Vinho de Cajú

9.11 Sabฉ̃o

$9122^{-e 1}$

9.13 Balaios

9.14 Carvão

sim

não

sim

$\square$ ñ̃o

$\left\{\begin{array}{l}\square \text { sim } \\ \text { não }\end{array}\right.$

$\left\{\begin{array}{l}\square \text { sim } \\ \square \text { ño }\end{array}\right.$

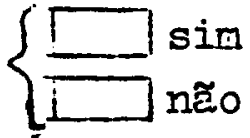

Quantas

Quentas

Quantas.

Quentas.

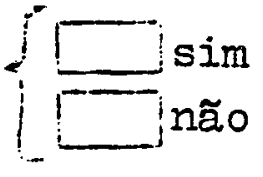

9.15 outros tipos de artesanato

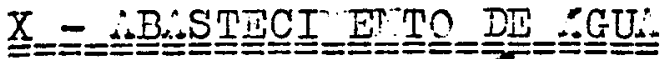

10.1 Abastecimento de Egua no tempo seco $\cdots$

$\left\{\begin{array}{l}\square \text { sim } \\ \square \text { não }\end{array}\right.$

$\square$ Bolanha.

Fonte (Lagoa) 
10.2 : quantidcie de kgua es $\left\{\begin{array}{l}\square \text { vita } \\ \square \text { Regular } \\ \square \text { ouca }\end{array}\right.$

10.3 jistancic entre a tabanca e o locel de abastecinento de śgua a is 5 .

10.4 Se houver furo na tabance. bombe funciona

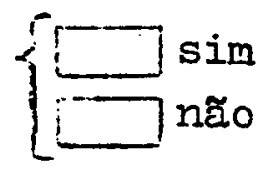

\section{YI}

11.I L.cesso E. tabanca por

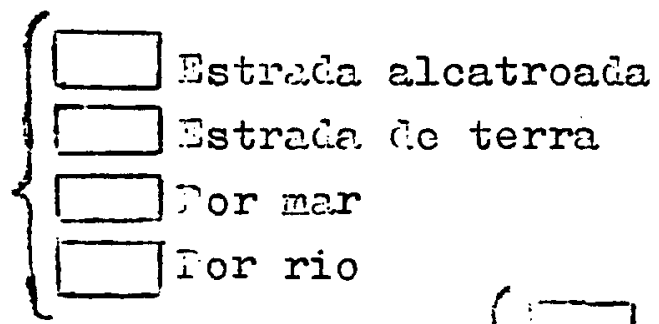

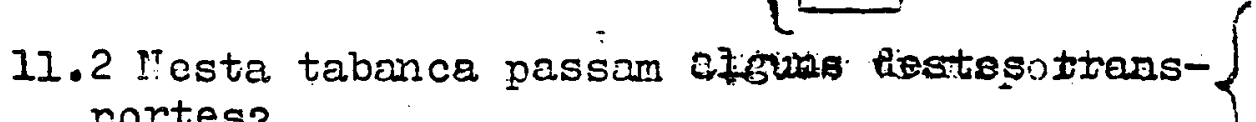
portes?

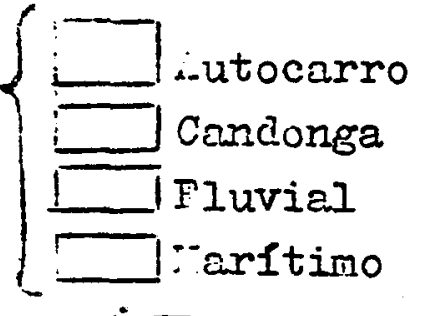

$11.3 \mathrm{Na}$ época las chuvas a tabanca fica isolr.je

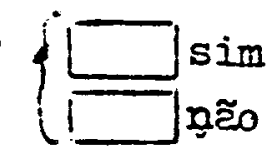

11.4 Se não há transporte ou se na época de chuva a tabanca fica isolada cleve-se ir até. *....... pis para o apanhar.

11.5 Há algum tipo de traç̧ão animal para transportar pessoas $\square$ im 11.6 Hé cenoas na tabance $\left\{\begin{array}{l}\square \text { sim } \\ \text { ñ̃o }\end{array}\right.$ Inão

11.7 Quantas.

12. Outras informações úteis soore a tabanca. 
REPUBLICA DA GUINE-BISSAO

\section{YINISTGRIO DO PLANO}

\section{GABINETE DO PLANO DA REGIÃO DE BOLAMA/BIJAGOS}

FICHA DE TABANCA

Tabanca

Número

\section{$\triangle \mathrm{RTESAN \perp TO}$}

1. Terrazias

$\square \operatorname{sim}$

Nẽo

$\square$ Não

$\square \operatorname{sim}$

2. Alfaiates :

$\square$ 苗o

$\square$ Não

3. Construtores de Casas:
Quantas

Numeros de Fereiros

Quantas

faz, ma s pode fazer

faz mais pode fazer
Dsim vuantas

$\square$ Não

WNão faz ma s pode fazer

4. Tecido:

$\square \operatorname{sim}$

Quantas-

$\int \mathrm{NaOO}$

WNao faz ma $s$ pode fazer

5. Cerâmica:

$\square$ Sim

$\square$ Não

$\square$ Não

Não faz ma s pode fazer

6. Construtores de Blocos(Dobe):

$\square \operatorname{sim}$

QNão

Quantas

Quantas

पnão

faz ma s pode fazer

7. Vinho de Palma: $\square$ sim

vuantas

$\square$ Não

$\square$ Não faz ma s pode fazer

8. Oleo de Palma: $\square$ Sim

Quantas

$\square$ Näo

$\square$ Nao faz ma s pode fazer

9. Sal:

] $\operatorname{sim}$

Não

$\square$ Não faz ma s pode fazer

10: Vinho de Cajú: $\square$ Sim

Quantas

Quantas

T Não

7 Não faz ma $s$ pode fazer

11. Sabão: 
12. Me1:

$\square$ sim

$\square$ Não

$\square$ Não faz ma s pode fazer

13. Balaios:

$\square$ sim

$\square$ Não

$\square$ Não faz ma.s pode fazer

14. Carvão

$\square \operatorname{sim}$

$\square$ Não

$\square$ Não faz ma s pode fazer

15. Carpinteiros: $\square$ sim

$\square$ Não

$\square$ Não faz ma s pode fazer quantas

Quantas

Quantas

Quantas

Quantas

$\square$ Não

$\square$ Nao faz ma s pode fazer

17. Artifice de côro:

18. Produção de cal: sim

$\square \operatorname{sim}$

Quantas

$\square$ Não

$\square$ Não faz ma s pode fazer

Quant as

$\square$ Não

$\square$ Não faz ma 3 pode fazer

19. Fumagem de peixe $\square$ sim

Quantas

$\square$ Não

Não faz ma $s$ pode fazer

20. Outros tipos de Artesanato:

PESCA:

Há Pescadores:

$\square$ sim

Quantas

$\square$ Não

Não pesca Motivo:

22. Pescadores pescam todo o ano: $\square$ sim Em que periodo: Seca $\square$ Chuva $\square$

23. Quais são principais métodos Utilizada na pesca?

D I V ERSOS

24. Há Associação de Tabanca? $\square$ sim

Não 
Qual tipo de Associação?

25. Há Projecto de Cooperação

$\square \operatorname{sim}$

$\square$ Não

- Qual tipo de Projecto?

26. Há Lctividades de produção que voces gostariam de ver desenvolvidas em tabanca?

$\square$ sim

- Qual actividades?

O B S ER V A C A $0:$

Data

$-/$

$/ 1987$

- Inquiridor 